\title{
Elucidating the Properties and Mechanism for Cellulose Dissolution in Tetrabutylphosphonium-Based Ionic Liquids using High Concentrations of Water
}

\author{
Brad Crawford \\ West Virginia University, crawfordb118@gmail.com
}

Follow this and additional works at: https://researchrepository.wvu.edu/etd

Part of the Applied Statistics Commons, Biochemical and Biomolecular Engineering Commons, Complex Fluids Commons, Numerical Analysis and Computation Commons, Numerical Analysis and Scientific Computing Commons, Other Chemical Engineering Commons, Polymer Science Commons, Probability Commons, Programming Languages and Compilers Commons, and the Transport Phenomena Commons

\section{Recommended Citation}

Crawford, Brad, "Elucidating the Properties and Mechanism for Cellulose Dissolution in Tetrabutylphosphonium-Based Ionic Liquids using High Concentrations of Water" (2020). Graduate Theses, Dissertations, and Problem Reports. 7641.

https://researchrepository.wvu.edu/etd/7641

This Dissertation is protected by copyright and/or related rights. It has been brought to you by the The Research Repository @ WVU with permission from the rights-holder(s). You are free to use this Dissertation in any way that is permitted by the copyright and related rights legislation that applies to your use. For other uses you must obtain permission from the rights-holder(s) directly, unless additional rights are indicated by a Creative Commons license in the record and/ or on the work itself. This Dissertation has been accepted for inclusion in WVU Graduate Theses, Dissertations, and Problem Reports collection by an authorized administrator of The Research Repository @ WVU.

For more information, please contact researchrepository@mail.wvu.edu. 


\title{
Elucidating the Properties and Mechanism for Cellulose Dissolution in Tetrabutylphosphonium-Based lonic Liquids using High Concentrations of Water
}

\section{Brad Crawford}

\author{
Dissertation submitted to the \\ Benjamin M. Statler College of Engineering and Mineral Resources \\ at West Virginia University
}

in partial fulfillment of the requirements for the degree of

Doctor of Philosophy in

Chemical Engineering

John W. Zondlo, Ph.D., Committee Chairperson

Richard Turton, Ph.D., P.E.

Christopher R. Iacovella, Ph.D.

Charter D. Stinespring, Ph.D.

Berend C. Rinderspacher, Ph.D.

Konstantinos A. Sierros, Ph.D.

Shuo Wang, Ph.D.

Department of Chemical and Biomedical Engineering

Morgantown, West Virginia

2020

Keywords: Molecular Dynamics Simulation, Ionic Liquid, Cellulose, Diffusion, Water, Biofuel, Biomass

Copyright 2020 Brad Crawford 


\title{
Abstract \\ Elucidating the Properties and Mechanism for Cellulose Dissolution in Tetrabutylphosphonium-Based Ionic Liquids using High Concentrations of Water
}

\author{
Brad Crawford
}

The structural, transport, and thermodynamic properties related to cellulose dissolution by tetrabutylphosphonium chloride $(\mathrm{TBPCl})$ and tetrabutylphosphonium hydroxide (TBPH)-water mixtures have been calculated via molecular dynamics simulations. For both ionic liquid (IL)-water solutions, water veins begin to form between the TBPs interlocking arms at $80 \mathrm{~mol} \%$ water, opening a pathway for the diffusion of the anions, cations, and water. The water veins allow for a diffusion regime shift in the concentration region from 80 to $92.5 \mathrm{~mol} \%$ water, providing a higher probability of solvent interaction with the dissolving cellulose strand. The hydrogen bonding was compared between small and large cellulose bundles, being 18 and 88 strands respectively. The dissolution of an 18 strand cellulose bundle was simulated in the $\mathrm{TBPCl}-$ water solution at various water concentrations. The Cl, TBP, and water enable cellulose dissolution by working together to form a cooperative mechanism for separating the cellulose strands from the bundle. The anions initially break the intra-strand hydrogen bonding and water helps delay the strand reformation. The TBP cation then can more permanently cleave the cellulose strand from the bundle. The TBP-peeling cellulose strand pairwise energy is net negative during the peeling process, indicating an energetically favorable process at moderate temperatures. The cellulose dissolution rapidly decays with increasing water concentration as the hydrogen bonding lifetimes for the chloride-cellulose hydroxyl hydrogens begin to fall below the lifetime of the largest cellulose intra-strand hydrogen bonds. The change in the diffusion regime occurs near the rapidly decaying dissolution region and may play a role in delaying the declining cellulose dissolution with increasing water concentrations. 


\section{Dedications}

I want to thank my family and friends for supporting me and granting me the time needed to pursue and complete my doctoral education. 


\section{Acknowledgments}

I would like to express my sincere gratitude to my late advisor Dr. Ahmed E. Ismail, for his guidance and instruction in the field of molecular dynamics simulations and allowing me to join his research group at West Virginia University.

I would like to sincerely thank my advisor, Dr. John W. Zondlo, for his guidance and expertise, reviewing my work, and providing feedback for my publications, which allowed me to finish my doctoral education and publish my work.

I would like to sincerely thank Dr. Richard Turton as he took over the National Science Foundation project, which I worked under, allowing me to finish this research and complete my doctoral studies.

I would like to sincerely thank Dr. Christopher R. Iacovella of Vanderbilt University, and Dr. Blake Mertz of West Virginia University as they each reviewed my papers, providing comments and suggestions, allowing me to publish my work.

I would like to thank the National Science Foundation for funding this research and sponsoring my doctoral education.

I want to thank the West Virginia University's High-Performance Computing (HPC) Research Centers for the use of the Spruce Knob and Thorny Flat supercomputing machine.

I want to thank Dr. Stefanos Papanikolaou for allowing the use of his computers on the HPC Research Centers to complete my simulations in a timely fashion.

I am honored to have Dr. John W. Zondlo, Dr. Richard Turton, Dr. Christopher R. Iacovella, Dr. Charter D. Stinespring, Dr. Berend C. Rinderspacher, Dr. Konstantinos A. Sierros, and Dr. Shuo Wang for my Ph.D. committee. I want to thank them for their time, commitment, and contributions to my doctoral education. 


\title{
Table of Contents
}

\author{
Abstract
}

Dedications

Acknowledgments $\quad$ iv

Abbreviations $\quad$ xiii

Atomic Labeling xiv

Variable Definitions $\quad$ XV

1 Introduction and Background 1

1.1 Ionic Liquids for Cellulose Dissolution . . . . . . . . . . . . . . . . . . 1

2 Methodology $\quad 7$

2.1 Property Simulation for the TBPCl-Water and TBPH-Water Systems . . . . 10

2.2 Cellulose Dissolution Simulations in TBPCl-water . . . . . . . . . . 15

2.2.1 Simulations of the Small $I \beta$ Cellulose Bundle (18 Strands) . . . . . . 21

2.2.2 Simulations of the Large $I \beta$ Cellulose Bundle (88 Strands) . . . . . . 22

3 Results $\quad 24$

3.1 Simulation Validation . . . . . . . . . . . . . . . . . . . . . . 24

3.1.1 Force Field Validation: TBPH/TBPCl-Water Densities . . . . . . . . 24

3.1.2 Small and Large I $\beta$ Cellulose Bundle Comparison in TBPCl-water . 25

3.2 Structural Properties . . . . . . . . . . . . . . . . . 33

3.2.1 Radial Distribution Functions (RDFs) for the TBPCl-Water Solution 33

3.2 .2 TBP and Water Nearest Neighbors and Clusters . . . . . . . . . . . . 37

3.3 Thermodynamic Data . . . . . . . . . . . . . . . . . . 43

3.3.1 Excess Properties: TBPH/TBPCl-Water Solutions . . . . . . . . . 43 
3.3.2 Heat Capacity and Thermal Expansivity: TBPH/TBPCl-Water . . . 44

3.4 Diffusion Properties: TBPH/TBPCl-Water Solutions . . . . . . . . . . . 48

3.5 Overview: Long Simulations with the Small I $\beta$ Cellulose Bundle in TBPCl-

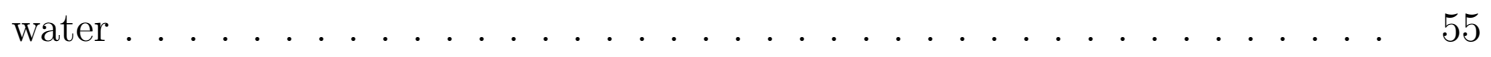

3.6 The Extent of Cellulose Dissolution in TBPCl-water . . . . . . . . . . . 55

3.6.1 Cellulose Dissolution in TBPCl-water . . . . . . . . . . . 56

3.6.2 Cellulose Strand Separation in TBPCl-water . . . . . . . . . . . . 57

3.7 Hydrogen bonding . . . . . . . . . . . . . . . . . 60

3.7.1 Hydrogen bonding: Only the TBPH/TBPCl-Water Solutions . . . . 60

3.7.2 Hydrogen Bonding Lifetimes in the TBPCl-water and Cellulose Solutions 62

3.8 Pairwise Energies and Hydrogen Bonding of the Peeling Strands in TBPCl-water 69

3.9 Cellulose Dissolution Mechanism in TBPCl-water . . . . . . . . . . . 74

3.10 Comparison between Alkylimidazolium IL's and TBPH / TBPCl-Water So-

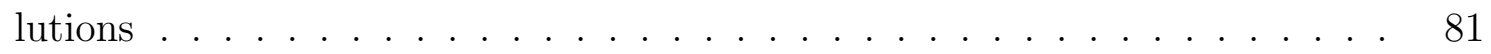

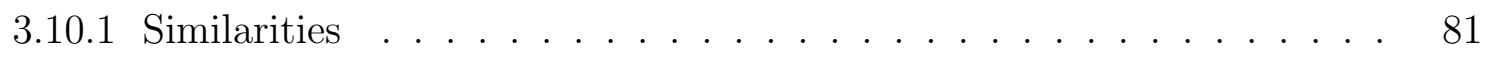

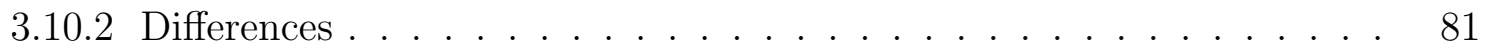

4 Discussion $\quad 84$

5 Conclusions $\quad 93$

$6 \quad$ Future Work $\quad 97$

7 Appendix $\quad 99$

7.1 Methodology (cont.) . . . . . . . . . . . . . . 100

7.2 Small and Large I $\beta$ Bundle Comparison in TBPCl-water (cont.) . . . . . . . 110

7.3 Radial Distribution Functions (RDFs) for TBPH-water Solution . . . . . . . 120

7.4 Water Vein Formation in the TBPH-water Solutions . . . . . . . . . . . . . 122

7.5 Diffusion Properties: TBPH-Water Solutions (cont.) . . . . . . . . . . . 125

7.6 Cellulose Dissolution in TBPCl-water (cont.) . . . . . . . . . . . . . 129

7.7 Cellulose Strand Separation in TBPCl-water (cont.) . . . . . . . . . . . . 130

7.8 Hydrogen Bonding Lifetimes in the TBPCl-water and Cellulose Solutions (cont.) 134 
7.9 Pairwise Energies and Hydrogen Bonding of the Peeling Strands in TBPClwater (cont.) . . . . . . . . . . . . . . . . . 137

7.10 Cellulose Dissolution Mechanism in TBPCl-water (cont.) . . . . . . . . . 148

8 General Reference to Past Publications Utilized in this Dissertation 150

$\begin{array}{lr}\text { References } & 151\end{array}$ 


\section{List of Figures}

1.1 Experimental cellulose solubility in TBPH-water and TBPCl-DMF . . . 4

1.2 Chemical structures of the molecules . . . . . . . . . . . . . 4

$2.1 \quad$ Atomic labeling for the cellulose strands . . . . . . . . . . . . . . . 19

$2.2 \quad$ Atomic labeling for TBP, $\mathrm{Cl}$, and water . . . . . . . . . 20

$2.3 \quad$ Initial configuration of the small $\mathrm{I} \beta$ cellulose bundle . . . . . . . . . . . 22

$2.4 \quad$ Initial configuration of the large $\mathrm{I} \beta$ cellulose bundle . . . . . . . . . 23

3.1 Simulated densities of the TBPH-water and TBPCl-water solutions . . 24

3.2 Coloring scheme for the hydrogen bonding comparison between the small and large cellulose bundles . . . . . . . . . . . . . . . . . . . . . . . . 29

3.3 Average inter-stand and intra-strand hydrogen bonding comparison between the small and large cellulose bundles at various water concentrations 30

3.4 Average solvent hydrogen bonding comparison between the small and large cellulose bundles at various water concentrations (Part 1 of 2) . . 31

3.5 Average solvent hydrogen bonding comparison between the small and large cellulose bundles at various water concentrations (Part 2 of 2) . . 32

3.6 TBPCl-water's Radial Distribution Functions (Part 1 of 2) . . . . . 34

$3.7 \quad$ TBPCl-water's Radial Distribution Functions (Part 2 of 2) . . . . 35

3.8 TBPs CT4-CT4 Radial Distribution Function for TBPCl-water . . . . 36

$3.9 \quad$ Avg. No. of nearest neighbors in the TBPCl/TBPH-Water solutions . . 37

$3.10 \quad$ Average largest clusters of water and $\mathrm{TBP}^{+}$ions . . . . . . . . . . 38

3.11 Depiction of molecules in the TBPCl-water solution . . . . . . . . 40

3.12 Water vein formation in TBPCl-water (Part 1 of 2 ) . . . . . . . . 41

3.13 Water vein formation in TBPCl-water (Part 2 of 2) . . . . . . . 42

$3.14 \quad$ Excess properties of the TBPH/TBPCl-water solutions . . . . . . . 44

3.15 Heat capacity $\left(c_{p}\right)$ of the TBPH/TBPCl-water solutions . . . . . . 46

3.16 Thermal expansivity $\left(\alpha_{\mathrm{p}}\right)$ of the TBPH/TBPCl-water solutions . . . 46

3.17 Anomalous diffusion coefficients in TBPCl-water . . . . . . . . . . 49

3.18 Anomalous diffusion coefficients in TBPH-water . . . . . . . . . . . 50 
3.19 Particle-averaged TAMSDs in TBPCl-water . . . . . . . . . . 51

3.20 Ergodicity breaking parameter in TBPCl-water . . . . . . . . . 52

3.21 MSD vs. particle-averaged TAMSD in TBPCl-water . . . . . . . . . . 54

3.22 The simulated cellulose dissolution concentrations for the TBPCl-water

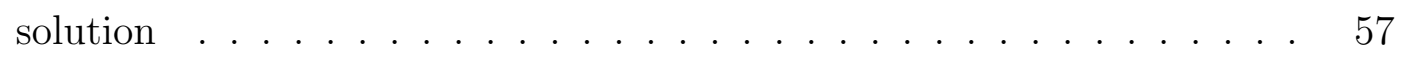

$3.23 \quad$ Maximum cellulose strand separation . . . . . . . . . . . . 59

3.24 Geometric specifications for hydrogen bonds . . . . . . . . . . 60

3.25 Average hydrogen bonds . . . . . . . . . . . . . . . . 61

3.26 The chloride's intra-strand hydrogen bond breaking lifetimes vs. the cellulose's intra-strand hydrogen bonding lifetimes. . . . . . . . . . . 64

3.27 The pairwise energies for the first yellow separating strand . . . . . 72

3.28 The hydrogen bonding for the first yellow separating strand (Part 1 of 2) 73

3.29 The hydrogen bonding for the first yellow separating strand (Part 2 of 2) 74

$3.30 \quad$ TBPCl-water molecular representations . . . . . . . . . . 75

3.31 The cellulose dissolution process for the $63.1 \mathrm{~mol} \%$ water concentration 78

3.32 The cellulose dissolution process in TBPCl-water . . . . . . . . . 79

3.33 The cellulose strand twisting for various concentrations . . . . . . 80

4.1 The summarized mechanism for cellulose dissolution in the TBPCl-water

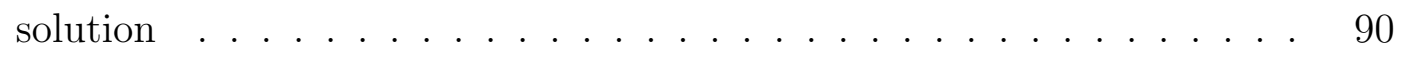

7.1 Average intra-strand hydrogen bonding comparison with standard deviations between the small and large cellulose bundles at various water

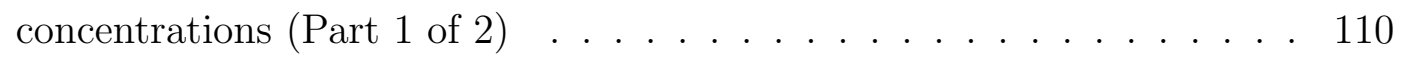

7.2 Average intra-strand hydrogen bonding comparison with standard deviations between the small and large cellulose bundles at various water

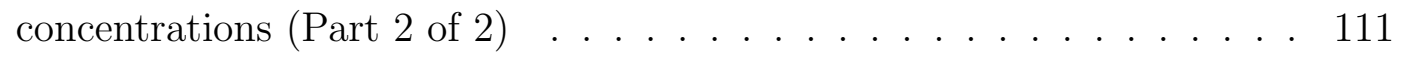

7.3 Average inter-strand hydrogen bonding comparison with standard deviations between the small and large cellulose bundles at various water concentrations $($ Part 1 of 2 ) . . . . . . . . . . . . . 112 
7.4 Average inter-strand hydrogen bonding comparison with standard deviations between the small and large cellulose bundles at various water concentrations $($ Part 2 of 2$) \ldots \ldots \ldots$

7.5 Average Cl-strand hydrogen bonding comparison with standard deviations between the small and large cellulose bundles at various water concentrations (Part 1 of 2$) \ldots \ldots \ldots$

7.6 Average Cl-strand hydrogen bonding comparison with standard deviations between the small and large cellulose bundles at various water

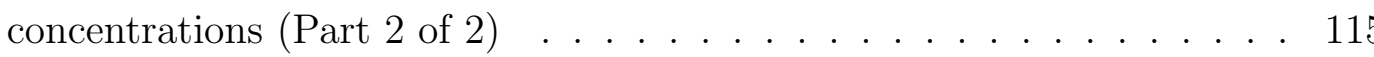

7.7 Average TBP-strand hydrogen bonding comparison with standard deviations between the small and large cellulose bundles at various water concentrations $($ Part 1 of 2$) \ldots \ldots \ldots \ldots$

7.8 Average TBP-strand hydrogen bonding comparison with standard deviations between the small and large cellulose bundles at various water

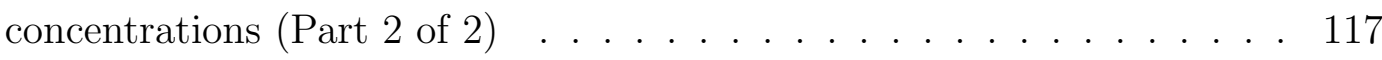

7.9 Average water-strand hydrogen bonding comparison with standard deviations between the small and large cellulose bundles at various water concentrations $($ Part 1 of 2$) \ldots \ldots \ldots \ldots$

7.10 Average water-strand hydrogen bonding comparison with standard deviations between the small and large cellulose bundles at various water

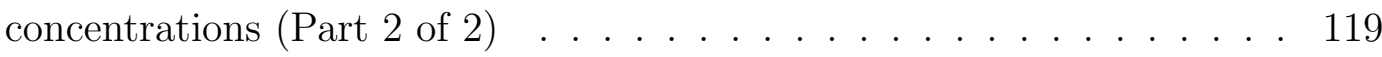

7.11 TBPH-water's Radial Distribution Functions (Part 1 of 2) $\ldots \ldots$

7.12 TBPH-water's Radial Distribution Functions (Part 2 of 2) . . . . 121

7.13 TBPs CT4-CT4 Radial Distribution Function for TBPH-water . . . . . 121

7.14 Depiction of Molecules in the TBPH-Water Solution . . . . . . . . . 122

7.15 Water vein formation in TBPH-water (Part 1 of 2$) \ldots \ldots . . \ldots 123$

7.16 Water vein formation in TBPH-water (Part 2 of 2$) \ldots \ldots . \ldots 124$

7.17 Ergodicity breaking parameter in TBPH-water . . . . . . . . . . 125

7.18 Particle-averaged TAMSDs in TBPH-water . . . . . . . . . . . 126

7.19 MSD vs. particle-averaged TAMSD in TBPH-water . . . . . . . . 127 
7.20 TBPCl-water small cellulose bundles dissolution measured by the number of dissolved glycans . . . . . . . . . . . . . . . . . . . . . 129

7.21 End view images of the cellulose bundle dissolution (Part 1 of 2) $\ldots 130$

7.22 End view images of the cellulose bundle dissolution (Part 2 of 2) . . . 131

7.23 Side view images of the cellulose bundle dissolution (Part 1 of 2) . . . 132

7.24 Side view images of the cellulose bundle dissolution (Part 2 of 2) $\ldots 133$

7.25 The pairwise energies for the second yellow separating strand . . . . 138

7.26 The hydrogen bonding for the second yellow separating strand (Part 1 of 2$) \ldots \ldots \ldots \ldots \ldots \ldots$

7.27 The hydrogen bonding for the second yellow separating strand (Part 2 of 2$) \ldots \ldots \ldots \ldots \ldots \ldots \ldots$

7.28 The pairwise energies and hydrogen bonding of the peeling strand(s) at 63.1 mol \% water . . . . . . . . . . . . . . . . . . 141

7.29 The pairwise energies and hydrogen bonding of the peeling strand(s) at 79.4 mol \% water . . . . . . . . . . . . . . . . . . 142

7.30 The pairwise energies and hydrogen bonding of the peeling strand(s) at $86.8 \mathrm{~mol} \%$ water . . . . . . . . . . . . . . . . . 143

7.31 The pairwise energies and hydrogen bonding of the peeling strand(s) at 91.1 mol \% water . . . . . . . . . . . . . . . . . . . . 144

7.32 The pairwise energies and hydrogen bonding of the peeling strand(s) at 93.9 mol \% water . . . . . . . . . . . . . . . . . . 145

7.33 The pairwise energies and hydrogen bonding of the peeling strand(s) at $95.8 \mathrm{~mol} \%$ water . . . . . . . . . . . . . . . . . 146

7.34 The pairwise energies and hydrogen bonding of the peeling strand(s) at $100 \mathrm{~mol} \%$ water . . . . . . . . . . . . . . . . . 147

7.35 Water diffusion in and out of the pocket between the cellulose strands . 148

7.36 The number of molecules between the first yellow peeling strand and the cellulose bundle . . . . . . . . . . . . . . . . . . . . . . . 149 


\section{List of Tables}

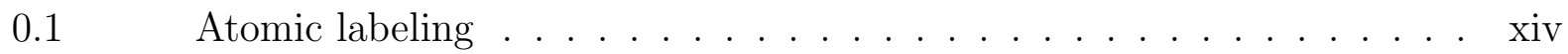

0.2 AMBER potential energy equation . . . . . . . . . . . $\quad$ xv

$0.3 \quad$ Anomalous diffusion equations . . . . . . . . . . . . . . . xv

2.1 Compositions of TBPH/TBPCl-water solvent simulations . . . . . . . 13

$2.2 \quad$ Small cellulose bundle (18 Strands) simulation compositions . . . . . 21

$2.3 \quad$ Large cellulose bundle (88 Strands) simulation compositions . . . . . . 23

$3.1 \quad$ Heat capacities and thermal expansivities of TBPH/TBPCl-water . . . 47

$3.2 \quad$ TBPCl anomalous diffusion coefficients . . . . . . . . . . . . . 50

$3.3 \quad$ TBPCl hydrogen bonding lifetimes (Part 1 of 5 ) . . . . . . . . . 67

$3.4 \quad$ TBPCl hydrogen bonding lifetimes (Part 2 of 5 ) . . . . . . . . 68

$7.1 \quad$ Atomic charges . . . . . . . . . . . . . . . 101

$7.2 \quad$ Pair coefficients . . . . . . . . . . . . . . . . 102

7.3 Bond coefficients (Part 1 of 2$) \ldots \ldots \ldots$

7.4 Bond coefficients (Part 2 of 2$) \ldots \ldots \ldots$. . . . . . . . . 104

7.5 Angle coefficients (Part 1 of 2 ) . . . . . . . . . . . 105

7.6 Angle coefficients (Part 2 of 2) . . . . . . . . . . 106

7.7 Torsion/dihedral angle coefficients (Part 1 of 3) . . . . . . . . 107

7.8 Torsion/dihedral angle coefficients (Part 2 of 3) . . . . . . . . . 108

7.9 Torsion/dihedral angle coefficients (Part 3 of 3) . . . . . . . . . 109

7.10 TBPH anomalous diffusion coefficients . . . . . . . . . . . . 128

7.11 Small cellulose bundle dissolution rates . . . . . . . . . . . . . 129

7.12 TBPCl hydrogen bonding lifetimes (Part 3 of 5) . . . . . . . . . 134

7.13 TBPCl hydrogen bonding lifetimes (Part 4 of 5) . . . . . . . . 135

$7.14 \quad$ TBPCl hydrogen bonding lifetimes (Part 5 of 5 ) . . . . . . 136 


\section{Abbreviations}

\begin{tabular}{ll}
\hline \hline Abbreviation & Definition \\
\hline$\alpha_{\mathrm{p}}$ & Thermal expansivity at constant pressure \\
Ac & Acetate \\
AMBER & Assisted Model Building with Energy Refinement \\
AMIMCl & 1-allyl-3-methylimidazolium chloride \\
BMIM & 1-butyl-3-methylimidazolium \\
$c_{p}$ & Heat capacity at constant pressure \\
DMF & Dimethylformamide \\
DMSO & Dimethylsulfoxide \\
EMIM & 1-ethyl-3-methylimidazolium \\
GLYCAM06 & Glycosylation-dependent Cell Adhesion Molecule 2006 \\
IL & Ionic Liquid \\
LAMMPS & Large-scale Atomic/Molecular Massively Parallel Simulator \\
MD & Molecular Dynamics \\
MSD & Mean Squared Displacement \\
MSDS & Material Safety Data Sheet \\
NPT & Constant molar-isobaric-isothermal \\
NVT & Constant molar-constant volume-isothermal \\
OH & Hydroxide or hydroxyl group \\
OPLS-AA & Optimized Potentials for Liquid Simulations-All Atom \\
PPPM & Particle-Particle Particle-Mesh \\
ReaxFF & Type of reactive force field \\
RESPA & Reversible reference System Propagator Algorithms \\
TAMSD & Time-averaged mean squared displacement \\
TBA & Tetrabutylammonium \\
TBACl & Tetrabutylammonium chloride \\
TBAH & Tetrabutylammonium hydroxide \\
TBP & Tetrabutylphosphonium \\
TBPCl & Tetrabutylphosphonium chloride \\
TBPH & Tetrabutylphosphonium hydroxide \\
TIP3P & Three-Site Transferrable Intermolecular Potential \\
TIP4P & Four-Site Transferrable Intermolecular Potential \\
VDW & Van der Waals \\
VMD & Visual Molecular Dynamics \\
\hline \hline
\end{tabular}




\section{Atomic Labeling}

\section{Table (0.1) Atomic labeling}

\begin{tabular}{ll}
\hline \hline Variable & Definition \\
\hline $\mathrm{A} \cdots \mathrm{H}-\mathrm{D}$ & hydrogen Acceptor atom $\cdots$ Hydrogen atom-hydrogen Donor atom \\
$\mathrm{Cx}$ & Carbon of location number $\mathrm{x}$ in the cellulose strand \\
$\mathrm{CTx}$ & Carbon of location number $\mathrm{x}$ in the TBP molecule \\
$\mathrm{Cl}$ & Chloride \\
$\mathrm{Hx}$ & Hydrogen of location number $\mathrm{x}$ in the cellulose strand \\
$\mathrm{HCx}$ & Hydrogen connected to CTx of the TBP molecule (x only ranges from 2 to 4) \\
$\mathrm{Hh}$ & Hydrogen of a hydroxide \\
$\mathrm{HP}$ & Hydrogen connected to CT1 of the TBP molecule \\
$\mathrm{Hw}$ & Hydrogen of water \\
$\mathrm{Oh}$ & Oxygen of a hydroxide \\
$\mathrm{Ox}$ & Oxygen of location number $\mathrm{x}$ in the cellulose strand \\
$\mathrm{Ow}$ & Oxygen of water \\
$\mathrm{P}$ & Phosphorous \\
\hline \hline
\end{tabular}




\section{Variable Definitions}

Table (0.2) AMBER potential energy equation

\begin{tabular}{|c|c|}
\hline Variable & Definition \\
\hline$E_{\text {total }}$ & Total potential energy \\
\hline$K_{r}$ & Potential energy bond constant \\
\hline$K_{\theta}$ & Potential energy angle constant \\
\hline$K_{n \phi}$ & The $n^{t h}$ potential energy torsion/dihedral angle constant \\
\hline$n$ & Number of maximums in the torsion/dihedral angle \\
\hline$q_{j}$ & Charge of atom $\mathrm{j}$ \\
\hline$q_{k}$ & Charge of atom $\mathrm{k}$ \\
\hline$r$ & Bond length \\
\hline$r_{j k}$ & Radial distance between atoms $j$ and $k$ \\
\hline$r_{0}$ & Equilibrium bond length \\
\hline$\gamma_{n}$ & The $n^{\text {th }}$ equilibrium torsion/dihedral angle \\
\hline$\epsilon_{j k}$ & Well depth of Lennard-Jones potential between atoms $j$ and $k$ \\
\hline$\theta$ & Bond angle \\
\hline$\theta_{0}$ & Equilibrium bond angle \\
\hline$\sigma_{j k}$ & Zero of Lennard-Jones potential between atoms $j$ and $k$ \\
\hline$\phi$ & Torsion/dihedral angle \\
\hline
\end{tabular}

Table (0.3) Anomalous diffusion equations

\begin{tabular}{ll}
\hline \hline Variable & Definition \\
\hline$t$ & Time \\
$\Delta$ & Lag time \\
$r_{i}$ & Center of mass of molecule $i$ \\
$<r^{2}(t)>$ & Mean squared displacement (MSD) \\
$\overline{\delta_{\mathrm{i}}^{2}}(\Delta)$ & Time-averaged mean squared displacement (TAMSD) of molecule $i$ \\
$<\overline{\delta^{2}}(\Delta)>$ & Particle-averaged TAMSD \\
$K_{\alpha}$ & Generalized diffusion coefficient \\
$\alpha$ & Anomalous diffusion exponent \\
$\chi$ & Ergodicity breaking parameter \\
\hline \hline
\end{tabular}




\section{Introduction and Background}

Cellulose is one of the most underutilized sources of energy in the world and resides within plant biomass. Biomass such as dead trees, tree limbs from logging operations, sawmill scraps, sawdust, leftover plant vines, or cornstalks are examples of cellulose-containing biomass, capable of being extracted and transformed into liquid biofuels, such as ethanol. ${ }^{1}$ Biofuels are part and parcel of a renewable, green, and sustainable energy future, and are an important part of the energy supply in the future. Liquid biofuels are a unique type of renewable energy, providing a portable energy-dense fuel, much like gasoline and diesel. The highly mobile nature of biofuels distinguishes them from other types of renewable energies, such as wind, solar, and tidal power, which are more capable of generating electricity in a fixed location. In 2018, over $98 \%$ of gasoline in the USA contained ethanol, and 10 volume \% of the total fuel usage was ethanol. ${ }^{2-5}$ While the percentage of ethanol in gasoline is low, the total ethanol production in 2018 exceeded 16 billion gallons. At the beginning of 2019, there were 199 existing ethanol refineries in the USA, with an additional 9 under construction $^{2,6,7}$ As the demand for ethanol grows, society should strive to find responsible and sustainable methods for future ethanol production. Currently, the missing step in the biomass conversion process is breaking down the biomass into its individual cellulose strands so it can efficiently be converted into fuels, such as ethanol, by enzymes or similar catalyzed reactions. $^{2,5}$

\subsection{Ionic Liquids for Cellulose Dissolution}

Ionic liquids (ILs) consist of a cation and anion. Typically ILs have low melting points, are non-flammable, practically non-volatile, and are thermally stable within a range of operating conditions. $^{1,8}$ ILs can also be combined with a co-solvent which can modify the properties of the solution by varying degrees depending on the concentration of the co-solvent. These ILs are highly dependent on the individual properties of their molecules, allowing a vast array of customizable ILs by scientifically designing the cation, anion, or co-solvent system.

To date, ILs have proven to be a valuable solvent for the chemical industry. ${ }^{2,9}$ However, many ILs can be expensive and toxic, which limits their viability in other markets. ${ }^{2,9-11}$ 
Therefore, it is crucial to minimize cost and toxicity by either adding a co-solvent to the IL, designing ILs that are less toxic, or both. Scientists can now use computational chemistry (i.e., molecular dynamics simulation, Monte Carlo simulations, or other simulations) to identify some of the critical properties of an IL which allow it to work in a specific process. These simulations may enable scientists to program automated machine learning algorithms to search for similar ILs with more desirable properties, such as those that are cheaper, environmentally friendly, and less toxic. Due to the high cost of experimental synthesis and testing, automated search methods could be a viable path to designing the next generation of IL co-solvent combinations, which minimize the cost, toxicity, and usability of the ILs. However, final experimental testing will be required to validate any simulation-derived IL co-solvent combinations.

Many solvents have been investigated to dissolve cellulose, but recently it has been demonstrated that ILs are capable of dissolving cellulose by disrupting the inter-strand (between strands) and intra-strand (within a strand) hydrogen bonding within the cellulose. ${ }^{1,8,12-18}$ Ideally, an IL should tolerate the presence of water when extracting cellulose from its biomass, as biomass can naturally contain up to $25 \mathrm{wt} \%$ water. Alternatively, the biomass could be industrially pre-dried, which would incur either a high energy penalty in the process line, or a significant capital expense for a storage area to sun-dry the biomass. Both the industrial pre-drying and natural sunlight drying processes consume additional time and resources in the manufacturing process, thereby decreasing the efficiency of the process and the economic viability of the fuel product.

While some ILs possess the ability to dissolve cellulose, the majority only do so at higher temperatures and become ineffective in the presence of low water concentrations. ${ }^{1,12-14,19}$ Alkylimidazolium-based ILs are among the most studied in the area of cellulose dissolution, and the literature provides detailed mechanisms for the dissolution process. ${ }^{1,12-14}$ The imidazolium-based ILs are a classic example of a high-temperature cellulose solvent, as they only work with low water content at temperatures of $433 \mathrm{~K}$ or above. ${ }^{1,12-14}$

At room temperature and on the high end of the water concentration range, experiments have shown that tetrabutylphosphonium hydroxide (TBPH)-water mixtures are capable of cellulose dissolution up to $20 \mathrm{wt} \%$ in 5 to 7 minutes. ${ }^{16}$ Within the fairly broad working range 
for of water concentrations, 86.8 to $93.9 \mathrm{~mol} \%$ water (30 to $50 \mathrm{wt} \%$ water), the TBPH-water mixture can dissolve at least $15 \mathrm{wt} \%$ cellulose in 5 to 7 minutes (see Figure 1.1a). ${ }^{16}$ For the single experimentally tested point, $93.9 \mathrm{~mol} \%$ water ( $50 \mathrm{wt} \%$ water), cellulose demonstrated no reactivity with the TBPH-water solution aside from hydrogen bonding, which implies that water concentrations above $93.9 \mathrm{~mol} \%$ should also produce a stable and unreactive solution. ${ }^{16,20}$ The TBPH-water and tetrabutylphosphonium chloride (TBPCl)-water properties simulations were limited to between $280 \mathrm{~K}$ and $320 \mathrm{~K}$, due to the thermal instability of the TBPH-water solution at temperatures of $323 \mathrm{~K}$ and higher. ${ }^{16}$ The TBPH-water solvent is known to react with itself at or below concentrations of $63 \mathrm{~mol} \%$ water, as the hydroxide reacts with the $\left(\mathrm{TBP}^{+}\right)$cation. ${ }^{16,21}$ However, this work considers TBPH-water and $\mathrm{TBPCl}$-water solutions between 50 to $100 \mathrm{~mol} \%$ water, so the structure of the system below $63 \mathrm{~mol} \%$ water can be analyzed before the TBPH-water solution decays. ${ }^{16,21}$

Burns et al. demonstrated that cellulose dissolution is possible using TBPCl in the co-solvent dimethylformamide (DMF). ${ }^{8}$ In pure $\mathrm{TBPCl}$, cellulose solubility is higher than $30 \mathrm{wt} \%$ cellulose, while falling to $0 \mathrm{wt} \%$ cellulose for pure DMF (see Figure 1.1b). ${ }^{8}$ The TBPCl-DMF co-solvent solution appears to have a curved cellulose solubility profile if plotted in mol \% cellulose, without a maximum away from the pure $\mathrm{TBPCl}$ concentration, unlike the skewed Gaussian profile for the TBPH-water solution. ${ }^{8,16}$ However, the TBPCl-DMF's cellulose solubility data only had three points which were taken at approximately $45 \mathrm{~K}$ higher than that for the TBPH-water solution, providing little clarity on the actual curve or the possible skewed Gaussian profile. ${ }^{8,16}$

The TBPCl-water solution was used as a surrogate to study cellulose dissolution in TBPwater based solutions, to remove any issue of thermal instability or reactivity with the TBPHwater solution. Since pure TBPCl is commercially available, it is likely thermally stable throughout the entire range of water concentrations while maintaining similar non-reactive characteristics of the TBPH-water solution. Using the TBPCl-water solution also allows the very slow kinetics in the molecular dynamics (MD) simulations to be accelerated, by using a simulation temperature of $360 \mathrm{~K}$, so that the cellulose dissolution process occurs using a reasonable amount of computational resources and time. The goal of this work is to provide general properties and attributes that enable the TBP-water class of ILs to dissolve cellulose 
at room temperature, so more efficient IL-water solutions can be designed in the future. As compared to alkylimidazolium-based ILs, the symmetrically branched structure of the TBP molecule seemingly contributes to the increased dissolution power of the TBPH-water and TBPCl-water systems at high water concentrations (see Figures 1.1 and 1.2). Up to now, there is little work exploring the mechanism that underlies TBPH's exceptional capability of cellulose dissolution under hydrated conditions.
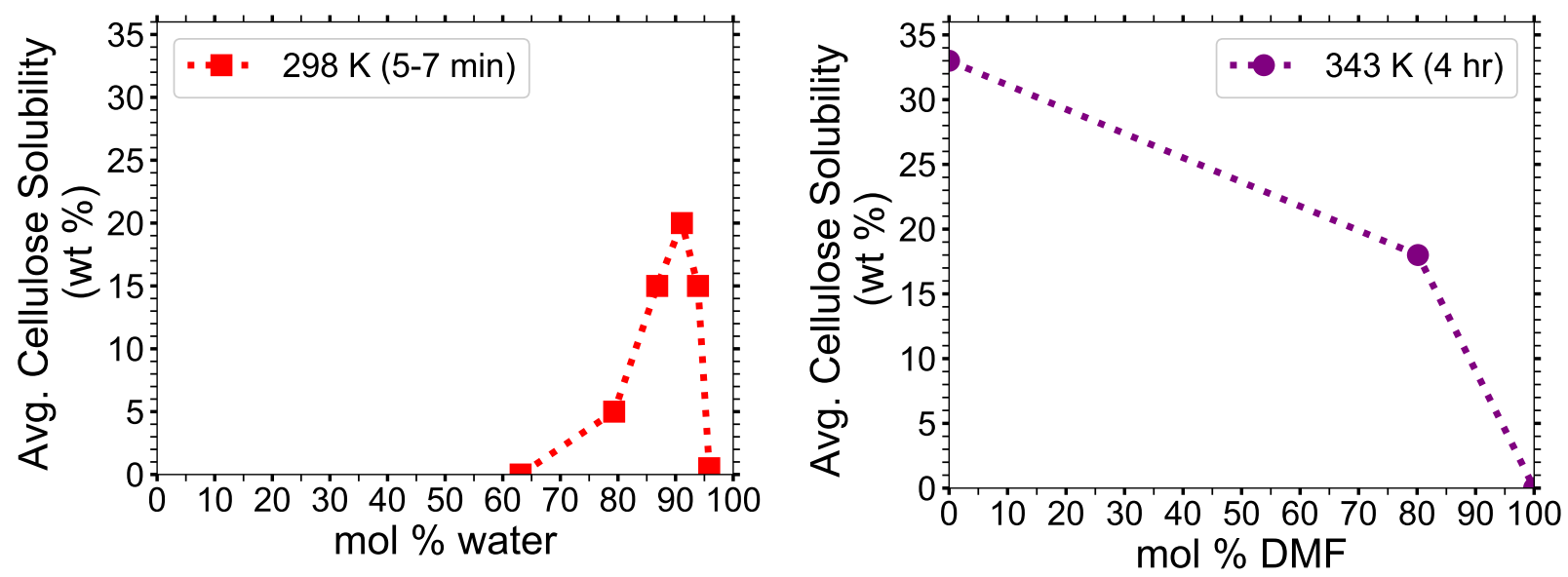

(a) TBPH-water solution at $298 \mathrm{~K}$ and $1 \mathrm{~atm}$ (b) TBPCl-DMF solution at $343 \mathrm{~K}$ and $1 \mathrm{~atm}$ (Data from Abe et al.) $)^{16}$ (Data from Burns et al. $)^{8}$

Figure (1.1) The experimental cellulose solubility in the TBPH-water and TBPCl-DMF solutions. ${ }^{22}$

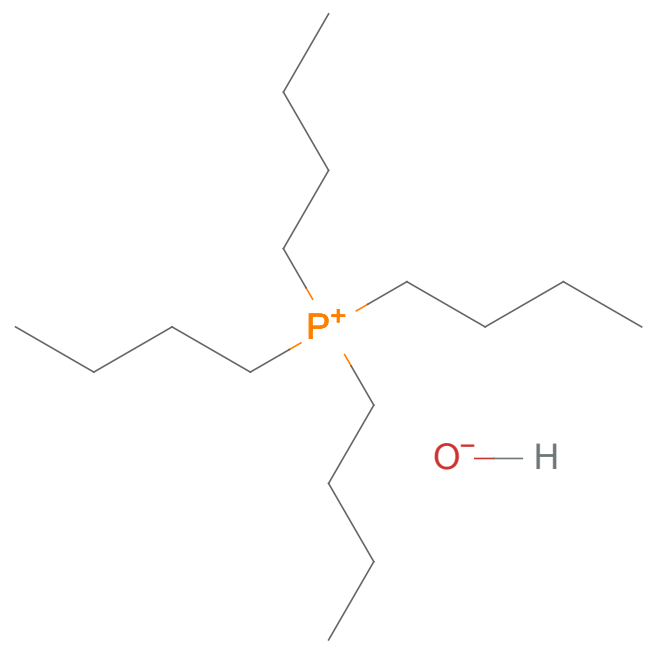

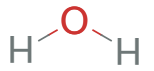

(b) Water

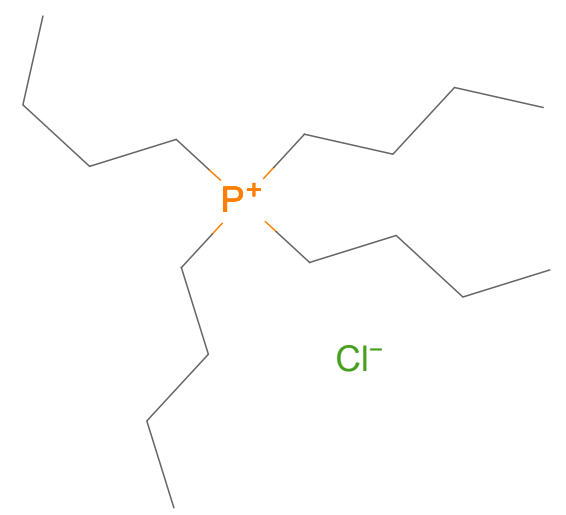

(c) $\mathrm{TBPCl}$

(a) $\mathrm{TBPH}$

Figure (1.2) Chemical structures of the molecules. ${ }^{23}$

In the following work, molecular dynamics simulations are used to evaluate the proper- 
ties of the TBPH-water and TBPCl-water solutions between $280 \mathrm{~K}$ and $320 \mathrm{~K}$, as well as the mechanism employed by the TBPCl-water solutions when dissolving cellulose at $360 \mathrm{~K}$. Specifically, the anomalous diffusion coefficients, density, excess molar volume, excess molar enthalpy of mixing, radial distribution functions (RDFs), heat capacity $\left(c_{p}\right)$, and thermal expansivity $\left(\alpha_{\mathrm{p}}\right)$. The small and large $I \beta$ cellulose bundle's inter-stand, intra-strand, and solvent-strand hydrogen bonding were compared using the Glycosylation-dependent Cell Adhesion Molecule 2006 (GLYCAM06) force field in the TBPCl-water solution. ${ }^{24}$ For the small cellulose bundle, the cellulose dissolution concentrations, mechanisms, strand separation distances, pairwise energies and hydrogen bonds between the solvent and the peeling or non-peeling cellulose strands, and the hydrogen bonding lifetimes of the solvent and the cellulose were determined in the TBPCl-water solution. This document begins with the methodology section, proceeded by the results section, and a comparison of the TBP-based solutions to the alkylimidazolium ILs. The discussion section follows, where some additional studies and comments are included. Finally, the document ends with the conclusions section.

\section{The unique contributions of this work include:}

1. TBPCl-water and TBPH-water solution properties

(a) The physical, thermodynamic, and diffusive properties were calculated. These were previously unavailable in the literature.

(b) The unique formation of water veins between 80 to $92.5 \mathrm{~mol} \%$ water has been elucidated. The water veins lead to increased diffusion of the solvent into the dissolving cellulose strands.

2. Cellulose dissolution in the TBPCl-water solution

(a) The intra-strand (within a strand), inter-strand (between strands), and TBPClwater (solvent)-strand hydrogen bonding between a small and large cellulose bundle (18 vs. 88 strands) were compared at various TBPCl-water concentrations, ensuring that the small cellulose bundle adequately exhibits the critical hydrogen bonding properties and can accurately simulate the dissolution behavior of the larger, more realistic bundle. The intra-strand and inter-strand hydrogen bonding 
were validated, since breaking the intra-strand hydrogen bonds is the first crucial step in the cellulose dissolution process. The solvent-strand hydrogen bonding was evaluated to verify that the solvent had the same interaction between the small and large cellulose bundles. Large cellulose bundles more accurately simulate the experimental properties of actual cellulose, but they are very computationally expensive, and the simulations can only be conducted over a short timeframe. Therefore, this study compares the size effects between a small and large cellulose bundle, ${ }^{25,26}$ ensuring that the small cellulose bundle adequately exhibits the critical hydrogen bonding properties, as seen in the larger bundle. This comparison warrants the use of the small cellulose bundle for the main simulations, which allows a much longer time to study the cellulose dissolution process. This type of comparison using an IL-water solution with the GLYCAM06 ${ }^{24}$ force field, or any other cellulose model, was not evaluated in the current literature.

(b) These simulations determined that cellulose dissolution is possible in the TBPClwater solution using high water concentrations at moderate temperatures. An approximate shape for the solubility profile was created. This result can help guide an experimental program.

(c) The TBP-peeling strand pairwise energies are consistently favorable (negative) during the cellulose dissolution process.

(d) A potential theoretical maximum co-solvent concentration may have been identified, which could determine the co-solvent concentration before the cellulose dissolution rapidly declines.

(e) Water plays a key role in cellulose dissolution by preventing cellulose strand reformation and allowing extra time for the TBP molecule to move in and more permanently separate the cellulose strand. 


\section{Methodology}

Molecular dynamics (MD) simulations evaluate a system by utilizing Newton's equations of motion as they are integrated through time. MD simulations can evaluate the thermodynamic, structural, and dynamic properties of a system, typically ranging from the femtosecond to microsecond time ranges. Classical MD simulations use potential energy equations, or force fields, to evaluate the system at the atomic level (i.e., every atom is accounted for and evaluated in the equations). There are different types of force fields, which are typically represented by a general analytical expression with various constants (see Equation 2.1). Many properties, such as the thermodynamic and structural properties, can be obtained in a single simulation with one starting trajectory via statistical time-averaging and ensuring the data are no longer time-correlated. ${ }^{27}$ Other dynamic properties like the binding of a drug molecule may require multiple simulations to be performed while averaging the data between them with each simulation starting at unique molecular positions or at least different molecular trajectories.

All of the MD simulations in this document were performed using the Large-scale Atomic / Molecular Massively Parallel Simulator (LAMMPS) package. ${ }^{28}$ The initial molecular configurations were constructed using the PACKMOL ${ }^{29}$ software (likely stands for Packing Molecules), which orients the initial configurations of the molecules via packing optimization. The visualizations, number of hydrogen bonds, and dihedral angles were generated

from the Visual Molecular Dynamics (VMD) software. ${ }^{30}$ The potential energy $E_{\text {total }}$ or force field uses the Assisted Model Building with Energy Refinement (AMBER) potential: ${ }^{31}$

$$
\begin{array}{r}
E_{\text {total }}=\sum_{\text {Bonds }} K_{r}\left(r-r_{0}\right)^{2}+\sum_{\text {Angles }} K_{\theta}\left(\theta-\theta_{0}\right)^{2}+\sum_{\text {Torsions }, n=1}^{3} K_{n \phi}\left(1-\cos \left(n \phi-\gamma_{n}\right)\right) \\
+\sum_{j=1}^{N} \sum_{k=j+1}^{N}\left\{4 \epsilon_{j k}\left[\left(\frac{\sigma_{j k}}{r_{j k}}\right)^{12}-\left(\frac{\sigma_{j k}}{r_{j k}}\right)^{6}\right]+\frac{q_{j} q_{k}}{r_{j k}}\right\}
\end{array}
$$

where $r_{0}, \theta_{0}$, and $\gamma_{n}$ are the equilibrium bond lengths, bond angles, and torsion/dihedral angles; $r, \theta$, and $\phi$ are the measured simulation bond lengths, bond angles, and torsion/dihedral angles; $r_{j k}$ is the measured simulation separation between atoms $j$ and $k$; and $q_{j}$ and $q_{k}$ are the 
charges of atoms $j$ and $k ; K_{r}, K_{\theta}$, and $K_{n \phi}$ are the bond, angle, and torsion/dihedral coefficients; and $\epsilon_{j k}$ and $\sigma_{j k}$ are the Lennard-Jones potential constants. The Lorentz-Berthelot ${ }^{32,33}$ (LB) mixing rules were utilized for the mixed $\sigma_{j k}$ and $\epsilon_{j k}$ coefficients between differing atoms:

$$
\begin{aligned}
\sigma_{j k} & =\frac{\left(\sigma_{j k}+\sigma_{j k}\right)}{2} \\
\epsilon_{j k} & =\sqrt{\epsilon_{j k} \epsilon_{j k}}
\end{aligned}
$$

The implemented force fields for the individual molecules throughout this document are from the following sources: the chloride anion was extracted from the work of Canongia Lopes et al., which derived an OPLS-AA/AMBER force field with the Lorentz-Berthelot mixing rules (note: these are also the same constants used for the OPLS-AA force field fitted to 68 unique ionic liquids by Sambasivarao et al. $) ;{ }^{32-35}$ the $\mathrm{TBP}^{+}$cation was taken from Zhou et $a l .{ }^{36}$ The other force fields that are specific to either the tetrabutylphosphonium hydroxide (TBPH)-water and tetrabutylphosphonium chloride (TBPCl)-water properties simulations or the cellulose dissolution simulations in TBPCl-water, are described in their particular Methodology section below. All the force field parameters can be found in the Appendix (see Tables 7.1, 7.2, 7.3, 7.4, 7.5, 7.6, 7.7, 7.8, and 7.9). The Isele-Holder method for calculating long-range dispersion forces was utilized in all the simulations with the LorentzBerthelot ${ }^{32,33}$ mixing rule (i.e., kspace modify mix/disp $=$ none in the LAMMPS code for the Isele-Holder method, since the number of different LJ diameters $\geq 7){ }^{28,37}$

Both the constant molar isobaric-isothermal (NPT) and constant molar isochoric- isothermal $(N V T)$ production runs are utilized in this work. All simulations used the Nosé-Hoover system $^{38-45}$ to control the pressure and temperature, with damping constants of 1000 for the pressure and 100 for the temperature, which means the pressure and temperature are relaxed in a timespan of $1000 \mathrm{fs}$ and $100 \mathrm{fs}$ for the pressure and temperature, respectively. The pressure dampening for the NPT parts was controlled by isotropically adjusting the simulation box. All NPT simulation pressures setpoints were $1 \mathrm{~atm}$, with the NVT simulation box sizes being the size of the last simulation step after the NPT stabilizations. The velocity Verlet algorithm ${ }^{46}$ was used for all the simulations. The SHAKE algorithm ${ }^{47}$ was utilized to maintain of the rigidity the $\mathrm{O}-\mathrm{H}$ bonds and fix the angle of the water molecules, 
and additionally, hold all other hydrogen-containing covalent bonds rigid.

For all the data and simulations in this work, the concentrations are represented as mol \% water, and this definition is clarified in Equation 2.4 for both the TBPH-water and TBPCl-water systems.

$$
\text { mol } \% \text { water }=\frac{(\text { molecules of water })(100)}{(\text { molecules of water })+(\text { molecules of TBPH or TBPCl })}
$$




\subsection{Property Simulation for the TBPCl-Water and TBPH-Water Systems}

The specific force fields for the individual molecules in the TBPH-water properties simulations were taken from the following works: the hydroxide anion parameters were obtained from Våcha et al.; ${ }^{48}$ and the four-site transferrable intermolecular potential (TIP4P)/2005 ${ }^{49}$ force field was used for water. Version 22-Feb-2018 of the LAMMPS software was used for these simulations.

Short-range dispersion and electrostatic-force cutoffs were set to $10 \AA$ for all non-bonded atoms. The long-range dispersion forces were computed using the Isele-Holder method ${ }^{37}$ with the Lorentz-Berthelot ${ }^{32,33}$ mixing rule and a real space accuracy of $10^{-4}$ and a kspace accuracy of $2 \times 10^{-3}$. The long-range electrostatic forces were calculated using the particleparticle particle-mesh (PPPM) method ${ }^{50}$ with an accuracy of $1 \times 10^{-5}$.

Both isobaric-isothermal (NPT) and constant volume-isothermal (NVT) production runs were conducted using approximately 10,000 atoms (see Table 2.1). In order to accelerate the simulation time, a 2-way split reversible reference system propagator algorithms (RESPA) method $^{51}$ was employed with an inner cutoff and outer cutoff of $4 \AA$ and $5 \AA$, respectively. The inner cutoff calculated the bond, angle, dihedral, improper, and pair-inner terms, while the outer cutoff calculated the pair-outer and Coulombic terms. These inner and outer cutoffs used timesteps of $2 \mathrm{fs}$ and $4 \mathrm{fs}$, respectively.

The simulations were conducted at $280 \mathrm{~K}, 300 \mathrm{~K}$, and $320 \mathrm{~K}$. To quickly acquire a thermal equilibrium, the molecules in the simulation were heated to $500 \mathrm{~K}$, followed by an equilibration period of $1 \mathrm{~ns}$. Then the molecules were cooled for $5 \mathrm{~ns}$ to $300 \mathrm{~K}$. The $280 \mathrm{~K}$ and $320 \mathrm{~K}$ simulations were obtained by heating or cooling the $300 \mathrm{~K}$ simulation for $1 \mathrm{~ns}$ and then allowing it to equilibrate for a few nanoseconds. The NVT simulations were restarted from the last atomic positions at the end of the NPT production runs. The NPT and NVT simulations were allowed to equilibrate for at least $4 \mathrm{~ns}$, while the $N P T$ and $N V T$ simulations collected the system data every $5 \mathrm{ps}$ and $2.5 \mathrm{ps}$, respectively.

Statistical convergence of the $55 \mathrm{~ns} N P T$ and $66 \mathrm{~ns} N V T$ production runs was confirmed using the multi-level blocking method of Flyvbjerg and Petersen ${ }^{27}$ for the last $50 \mathrm{~ns}$ and 
$60 \mathrm{~ns}$, respectively. The last $50 \mathrm{~ns}$ and $60 \mathrm{~ns}$ were used for the production runs of the NPT and NVT simulations, respectively. The data were time-averaged for the $280 \mathrm{~K}, 300 \mathrm{~K}$, and $320 \mathrm{~K}$ to compute all the presented data at constant pressure. This time-averaging between the three temperatures was also used to compute the constant pressure heat capacities at $300 \mathrm{~K}, c_{p}=(\partial H / \partial T)_{p}$, via a linear approximation method. ${ }^{52}$ The linear approximation method was utilized to determine the thermal expansivities at $300 \mathrm{~K}, \alpha_{p}=v^{-1}(\partial v / \partial T)_{p}$. The anomalous diffusion coefficients, radial frequency distributions (RDFs), hydrogen bonds, water and TBP clustering, were all computed using the entire $60 \mathrm{~ns}$ of the $N V T$ productions runs. VMD ${ }^{30}$ was directly used to calculate the RDFs and the number of hydrogen bonds. This study was conducted using only one simulation at each concentration and temperature. Therefore, it is appropriate to mention the possibility of data sensitivity, bias, and larger errors by only using a single starting configuration or trajectory for each individual the simulations. However, each separate concentration started at a unique configuration and trajectory. Additionally, with increased computational resources, larger systems could be simulated, which could minimize the data sensitivity and error in the analysis.

To visualize the simulation structures in more detail, the water vein pictures for both TBPH-water and TBPCl-water simulations are from a much larger system of approximately 100,000 atoms. These larger simulations employed the three-site transferrable intermolecular potential (TIP3P)-pppm water model ${ }^{53,54}$ and utilized the same LAMMPS ${ }^{28}$ parameters as listed for the cellulose dissolution simulations in TBPCl-water. The TBPH-water and TBPCl-water simulations were only run at $320 \mathrm{~K}$ and $360 \mathrm{~K}$, respectively. The short-range electrostatic and dispersion force cutoffs were carefully reduced in the larger simulations by ensuring the system density did not change by more than $0.5 \%$ from the smaller simulations. The reduced cutoffs were required to decrease the future cellulose bundle simulation time by $20 \%$. There is no notable visual difference when comparing them to the smaller TIP4P/2005 simulations at $300 \mathrm{~K},{ }^{49}$ but the larger simulations provided a more detailed picture of the water veins/channeling structures. These larger TIP3P-pppm simulations are preferred visually, as it is easier to see the patterns that form as the water concentration increases. ${ }^{53,54}$

The diffusion regime is determined by the anomalous diffusion coefficients, specifically the 
anomalous diffusion exponent $(\alpha)$ in Equation 2.8. The regime is subdiffusive if $0<\alpha<1$, normal diffusive if $\alpha=1$, and superdiffusive if $\alpha>1 .^{55}$ The generalized diffusion coefficient $\left(K_{\alpha}\right)$ sets the magnitude of the diffusion regime. ${ }^{55}$ The time-averaged mean squared displacement (TAMSD or $\overline{\delta_{\mathrm{i}}^{2}}(\Delta)$ ) was utilized in addition to the mean squared displacement (MSD or $\left\langle r^{2}(t)>\right.$ ), as lower water concentrations exhibited subdiffusive properties. ${ }^{55}$ The generalized diffusion coefficient $\left(K_{\alpha}\right)$ and the anomalous diffusion exponent $(\alpha)$ were calculated by fitting the particle-averaged TAMSDs $\left(<\overline{\delta^{2}}(\Delta)>\right)$. In this system, the particle-averaged TAMSD is a smoothed and nearly identical version of the MSD, which is explained later in more detail. The ergodicity breaking parameter $(\chi)$ is also evaluated, with the definition from Grebenkov et al. ${ }^{56}$ (see Equation 2.9). The MSD, TAMSD, particle-averaged TAMSD, ergodicity breaking parameter, and anomalous diffusion equations are as follows:

$$
\begin{gathered}
M S D=<r^{2}(t)>=\frac{1}{N} \sum_{i=1}^{N}\left|r_{\mathrm{i}}(t)-r_{\mathrm{i}}(0)\right|^{2} \\
T A M S D=\overline{\delta_{\mathrm{i}}^{2}}(\Delta)=\frac{1}{t-\Delta} \int_{0}^{t-\Delta}\left|r_{\mathrm{i}}(T+\Delta)-r_{\mathrm{i}}(T)\right|^{2} d T \\
<\overline{\delta^{2}}(\Delta)>=\frac{1}{N} \sum_{i=1}^{N} \overline{\delta_{\mathrm{i}}^{2}}(\Delta) \\
<\overline{\delta^{2}}(\Delta)>=K_{\alpha} \Delta^{\alpha} \\
\chi \frac{\operatorname{Var}\left[\overline{\delta_{\mathrm{i}}^{2}}(\Delta)\right]}{\left(\operatorname{Mean}\left[\overline{\delta_{\mathrm{i}}^{2}}(\Delta)\right]\right)^{2}}
\end{gathered}
$$

where $\mathbf{r}_{\mathrm{i}}$ represents the approximate center of mass of molecule $i$. The lag time, $\Delta$, is represented in the TAMSD equations, which are the width of time windows moving along the displacement function. ${ }^{55}$ The spacing between the time-averaged trajectories was 0.025 ns. In these calculations, the center of mass was approximated using the central $\mathrm{P}$ atom for the $\mathrm{TBP}^{+}$cation, and the $\mathrm{O}$ atom for the hydroxide ion and water molecule. The center of mass approximations were sufficient, as the actual center of mass only showed minimal deviations from the utilized atoms. Specifically, for the TBP molecule, only minimal 
deviations averaging less than $0.5 \AA$ occurred from the center of the $\mathrm{P}$ atom under energy minimized conditions for several tested configurations in Avogadro (version 1.2.0), ${ }^{57}$ likely due to its symmetric shape and heavy central weighting. The generalized diffusion coefficient and the anomalous diffusion exponent were calculated separately for each identical ion or molecule in the simulation from 0 to $60 \mathrm{~ns}$, using particle-averaged TAMSD (see Equation 2.8).

Table (2.1) Compositions of TBPH/TBPCl-water systems simulated in this study.

\begin{tabular}{ccccc}
\hline \hline $\begin{array}{c}\text { Mole \% } \\
\text { water }\end{array}$ & $\begin{array}{c}\text { Total } \\
N_{\mathrm{IL}}\end{array}$ & $\begin{array}{c}\text { Total } \\
N_{\mathrm{H}_{2} \mathrm{O}}\end{array}$ & $\begin{array}{c}\text { Total } \\
\text { molecules }\end{array}$ & $\begin{array}{c}\text { Total } \\
\text { atoms }\end{array}$ \\
\hline $0.55^{a}$ & 180 & 1 & 181 & 9903 \\
5.26 & 180 & 10 & 190 & 9930 \\
10.0 & 180 & 20 & 200 & 9960 \\
20.0 & 180 & 45 & 225 & 10035 \\
30.0 & 180 & 77 & 257 & 10131 \\
40.0 & 180 & 120 & 300 & 10260 \\
50.0 & 170 & 170 & 340 & 9860 \\
60.0 & 165 & 248 & 413 & 9819 \\
70.0 & 160 & 374 & 534 & 9922 \\
80.0 & 150 & 600 & 750 & 10050 \\
85.0 & 140 & 794 & 934 & 10082 \\
90.0 & 120 & 1080 & 1200 & 9840 \\
$92.5^{b}$ & 110 & 1357 & 1467 & 10121 \\
$94.0^{b}$ & 100 & 1567 & 1667 & 10201 \\
95.0 & 90 & 1710 & 1800 & 10080 \\
99.0 & 30 & 2970 & 3000 & 10560 \\
$99.97^{a}$ & 1 & 3000 & 3001 & 9055 \\
\hline \hline
\end{tabular}

${ }^{a}$ Infinite-dilution simulations.

${ }^{b}$ Only $N V T$ simulations were performed at these concentrations. ${ }^{58}$

While the TBPH-water mixture dissolves cellulose at room temperature and at $91.1 \mathrm{~mol} \%$ water ( $40 \mathrm{wt} \%$ water), it is known to be thermally unstable at temperatures higher than 323K. ${ }^{16}$ Therefore, the simulations in this work are limited to temperatures between $280 \mathrm{~K}$ and $320 \mathrm{~K}$. At concentrations below $63 \mathrm{~mol} \%$ water, the TBPH-water system is known to decompose, as the hydroxide reacts with the tetrabutylphosphonium $\left(\mathrm{TBP}^{+}\right)$cation. ${ }^{16,21}$ The TBPH solution showed no reactivity with cellulose aside from hydrogen bonding at $93.9 \mathrm{~mol} \%$ water ( $50 \mathrm{wt} \%$ water), which was the only concentration tested, suggesting, that 
higher water concentrations would also be unreactive. ${ }^{16,20}$ The TBPH-water solution could be reactive at low water concentration, which is discussed in more detail later; however, these non-reactive results provide viable reasons for the increased power of cellulose dissolution in the presence of water. $\mathrm{TBPCl}$ is sold as a pure salt, so it should be thermally stable throughout any range of water concentration, while maintaining its cellulose solubility and co-solvent effects. In this work, the simulation range is limited from 50 to 99.97 mol\% water. Data are presented at slightly below the stability range (50 to $60 \mathrm{~mol} \%$ water), showing how the structure would look before it decays, as in the case of TBPH.

The critical water concentration range for cellulose solubility ( 85 to 92.5 mol \% water), ${ }^{16}$ as shown in Figure 1.1, has a unique set of structural and physical properties not found at other concentrations. Figure 1.1 also correlates these structural and chemical changes with the experimental data, which are highlighted in this experimentally optimal concentration range in the figures throughout this paper. The chemical structures of TBPH, TBPCl, and water are shown in Figure 1.2. 


\subsection{Cellulose Dissolution Simulations in TBPCl-water}

Tetrabutylphosphonium chloride ( $\mathrm{TBPCl})$-water mixtures were used to simulate the dissolution of a I $\beta$ cellulose bundle via all-atom molecular dynamics. There are many different cellulose types defined by their molecular configurations, such as cellulose $\mathrm{I} \alpha, \mathrm{I} \beta, I I, I I I_{I}$, and other crystalline phases. In plants, cellulose occurs in both the $\mathrm{I} \alpha$ and $\mathrm{I} \beta$ crystalline phases and are suspected to exist mostly in the $\mathrm{I} \beta$ crystalline phase. ${ }^{25}$ Both the $\mathrm{I} \alpha$ and $\mathrm{I} \beta$ crystalline phases are known to be stable in water at low to moderate temperatures. The I $\beta$ crystalline phase appears to be more stable in IL solutions, due to the differing molecular configurations and hydrogen bonding within the cellulose bundle. ${ }^{15}$ The I $\beta$ crystalline phase was selected since it is the most dominant in plant-based cellulose and due to its higher stability in ILs. ${ }^{15,25}$ The simulations were performed using the 12-Dec-2018 version of the Large-scale Atomic/Molecular Massively Parallel Simulator (LAMMPS) software. ${ }^{28}$ The PACKMOL software was utilized to generate the initial packing configurations for the simulations, ${ }^{29}$ while the initial structure of the $\mathrm{I} \beta$ - cellulose bundle was built by means of the Cellulose-Builder code. ${ }^{59}$ The large $\beta$-cellulose bundle contains 88 individual strands and 24 glycans (24 glucose units) per strand because it was previously determined that the 81 strand bundle with 20 glycans closely matched the actual experimental twisting data with approximately one percent more cellulose bundle twisting in the simulations. ${ }^{25}$ The small I $\beta$ cellulose bundle contains 18 individual strands and 12 glycans (12 glucose units) per strand. The small bundle size was selected because it was larger than a previous small bundle, from a study which had 10 strands and 8 glycans per strand. ${ }^{15}$ However, this small bundle size made these long simulations at various water concentrations computationally feasible. The TBPCl-water concentrations are based on experimental data from the TBPH-water system (see Tables 2.2 and 2.3). ${ }^{16}$ The TBPCl-water simulation boxes were designed to provide the cellulose with at least a $25 \AA$ or greater distance through the periodic boundary condition. The cellulose dissolution concentrations are presented in wt \% dissolved cellulose (see Equation 2.10). 


$$
\text { wt } \% \text { dissolved cellulose }=\frac{(\text { dissolved cellulose } w t)(100)}{(\text { dissolved cellulose } w t)+(\text { water } w t)+(T B P C l w t)}
$$

The additional force field constants for the simulations were taken from the following sources: the Glycosylation-dependent Cell Adhesion Molecule 2006 (GLYCAM06) force field was employed for the cellulose bundle; ${ }^{24}$ and the three-site transferrable intermolecular potential (TIP3P)-pppm model was selected for water. ${ }^{53,54}$ Lorentz-Berthelot mixing rules were employed for mixing the force fields. ${ }^{32,33}$ The potential energy equations are from the Assisted Model Building with Energy Refinement (AMBER) potential. ${ }^{31}$

Timesteps of $2 \mathrm{fs}$ were utilized with the velocity Verlet algorithm, ${ }^{46}$ extracting data every 10 ps. The short-range dispersion and electrostatic forces for all non-bonded atoms had cutoffs of $8 \AA .58$ The long-range electrostatics calculations used the Particle-Particle-ParticleMesh (PPPM) method, ${ }^{50}$ with an accuracy of $10^{-4} \cdot{ }^{58}$ The Isele-Holder method calculated the long-range dispersion forces, ${ }^{37}$ with a real space accuracy of $10^{-3}$ and a kspace accuracy of $2 \times 10^{-2}{ }^{58}$ These short-range cutoffs and long-range parameters were validated using smaller TBPCl-water simulations, approximately 10,000 atoms, with short-range dispersion and electrostatic force cutoffs of $10 \AA$ for all non-bonded atoms. The simulations with the $10 \AA$ cutoffs utilized more accurate long-range dispersion force calculations, with a real space accuracy and kspace accuracy of $10^{-4}$ and $2 \times 10^{-3}$, respectively. ${ }^{37}$ The TBPCl-water simulations with 10,000 atoms were also conducted using short-range dispersion and electrostatic force cutoffs of $8 \AA$ for all non-bonded atoms, utilizing the same parameters in this study. All the simulations used the same PPPM accuracy. The production runs for this comparison were simulated using the NPT ensemble for $50 \mathrm{~ns}$ to get the density, and the $N V T$ ensemble for $60 \mathrm{~ns}$ to calculate the chloride-water hydrogen bonding. The density of the system and chloride-water hydrogen bonding between the $8 \AA$ and $10 \AA$ short-range cutoff simulations were compared at 60, 70, 90, and $99.97 \mathrm{~mol} \%$ water. For the 60, 70, 90, and $99.97 \mathrm{~mol} \%$ water concentrations, the density of the system varied $0.01,0.02,0.15$, and 0.12 percent, while the chloride-water hydrogen bonding varied 2.7, 1.7, 0.9, 0.6 percent, respectively. The short-range cutoff of $8 \AA$ yielded variations that were within with the density and 
property deviations from the Isele-Holder method with reasonable tolerances. ${ }^{37}$ The shortrange cutoffs of $8 \AA$ reduced the computational cost of the simulations by approximately half, thereby allowing these long simulations to be conducted using the largest possible cellulose bundles at various water concentrations. It was theorized that for the Isele-Holder method, the short-range cutoffs in many systems could be reduced while providing accurate results, as long as the short-range cutoff was not smaller than twice the largest LennardJones diameter, which agrees with these results. ${ }^{37}$ The Nosé-Hoover system controlled the temperature and pressure with damping constants of 100 and 1000, respectively. These damping constants translate to relaxing the temperature and pressure every 100 fs and 1000 fs, respectively. ${ }^{38-45,58}$ The simulations box size was modified isotropically with respect to the pressure damping. The SHAKE algorithm held the $\mathrm{O}-\mathrm{H}$ bonds and angle of the water molecules rigid, along with any other covalent bonded hydrogens in the TBP or cellulose molecules. ${ }^{47}$ The AMBER 1-4 interaction scaling factors from Cornell et al. were utilized for the TBP molecules, ${ }^{31}$ and the 1-4 interaction scaling factors of unity (i.e., 1) were applied for the cellulose's GLYCAM06 force field. ${ }^{24}$

Using a constant molar-isobaric-isothermal (NPT) ensemble, the TBPCl-water simulations were heated for $4 \mathrm{~ns}$ to $500 \mathrm{~K}$, cooled to $360 \mathrm{~K}$ over $4 \mathrm{~ns}$, then allowed to equilibrate at $360 \mathrm{~K}$ for at least $3 \mathrm{~ns}$. In order to insert the cellulose into the TBPCl-water system without overlapping atoms, all non-cellulose atoms were deleted if within $2 \AA$ from the cellulose bundle. ${ }^{15}$ Using the NPT ensemble, the TBPCl-water and cellulose simulations were started at $5 \mathrm{~K}$ and heated to $360 \mathrm{~K}$ over $1 \mathrm{~ns}$, ensuring that the cellulose bundle remains in its most stable configuration. Once the TBPCl-water and cellulose system reached $360 \mathrm{~K}$, the molecules were allowed to equilibrate for $4 \mathrm{~ns}$, before moving the simulation to the constant molarconstant volume-isothermal $(N V T)$ ensemble for the rest of the production simulations. A single simulation was conducted for each concentration.

LAMMPS does not support pairwise energy calculations when a system has mixed 1-4 interaction scaling factors or uses the Isele-Holder method for long-range dispersion forces. ${ }^{28,37}$ The 1-4 interaction scaling factors must be zero to prevent unrealistic positive pairwise energies, since the bond, angle, and dihedral energies are not calculated in the same molecule, specifically the peeling cellulose strand. The bundle-peeling strand does calculate the pair- 
wise energies from within its peeling strand provided they are 1-5 interactions or further, while the other analyses which are comparing differing molecules do not. The 1-2 and 1-3 interaction scaling factors were also zero, but this was the same as in all the simulations and re-runs. Therefore, the following calculation parameters were changed to compensate for this during the pairwise energies re-run data analysis: the pairwise energy calculations were set to zero for the 1-4 interaction scaling factors; the dispersion and electrostatic forces for all non-bonded atoms had cutoffs of $20 \AA$; no long-range dispersion forces were calculated; and the PPPM method was utilized for calculating long-range electrostatic forces. ${ }^{50}$ The shortrange dispersion force cutoffs were extended by 2.5 times (i.e., to $20 \AA$ ) to compensate for the removal of the long-range dispersion calculations; otherwise, the pairwise energies for the system could not be calculated. In many molecular dynamics simulations, the long-range dispersion force are not used at all, with the standard short-range dispersion force cutoffs being around 10 to $12 \AA$.

A hydrogen bond exists between the hydrogen acceptor and hydrogen if they are within $2.45 \AA$, and the hydrogen-donor-acceptor angle is $30^{\circ}$ or less. ${ }^{15,60-68}$ The above conditions mandate that the donor-acceptor distance is $3.5 \AA$ or less. ${ }^{15,60-68}$ The calculated intra-strand (within a strand) hydrogen bonding in the cellulose does not include all the intra-strand bonding, and only includes the primary intra-strand bonding that is present in the experimental data (see Figure 2.1). ${ }^{69}$ The inter-strand (between strands) hydrogen bonding in the cellulose does represent all the hydrogen bonds between other cellulose strands. The atomic labeling for all the atoms in the system are represented in Figures 2.1 and 2.2. 


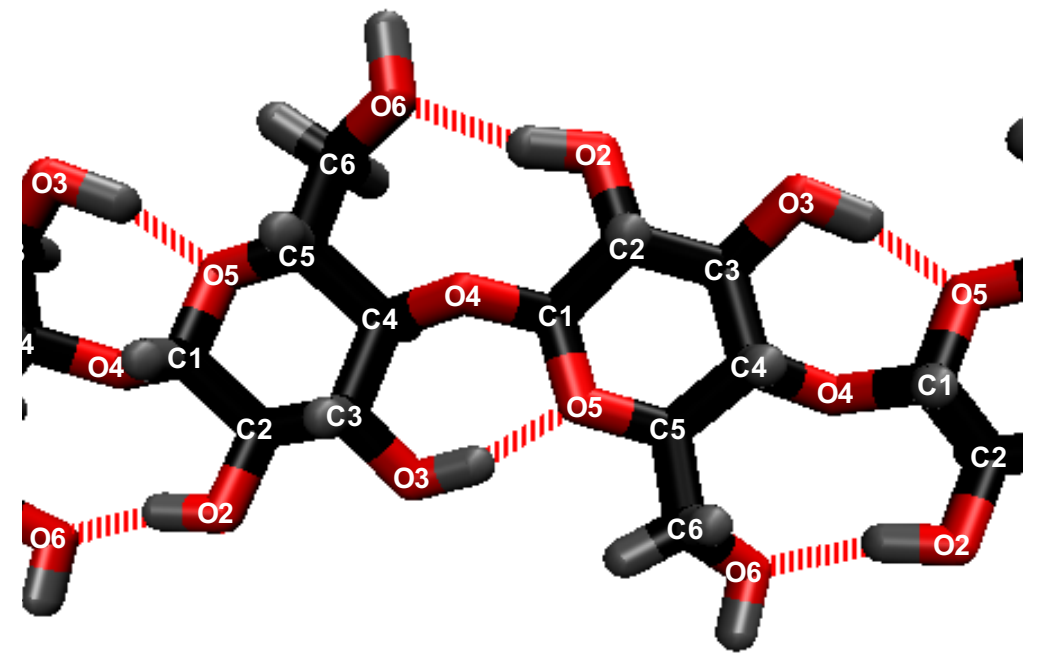

Figure (2.1) Atomic labeling for the cellulose strands. The black, red, and gray atoms are carbons, oxygens, hydrogens, respectively. The dashed lines are hydrogen bonding within the cellulose strand or intra-strand hydrogen bonding. For clarity, only the carbons and the oxygens are labeled in the cellulose strands. In the cellulose strands: the hydrogens bonded to the oxygens are named $\mathrm{HO} 2, \mathrm{HO} 3$, and $\mathrm{HO} 6$, corresponding to the $\mathrm{O} 2$, O3, and O6 oxygens; the hydrogens bonded to the carbons are named H1, H2, H3, H4, H61, and $\mathrm{H} 62$, corresponding to the $\mathrm{C} 1, \mathrm{C} 2, \mathrm{C} 3, \mathrm{C} 4, \mathrm{C} 6$, and $\mathrm{C} 6$ carbons, respectively. ${ }^{22,30}$ 


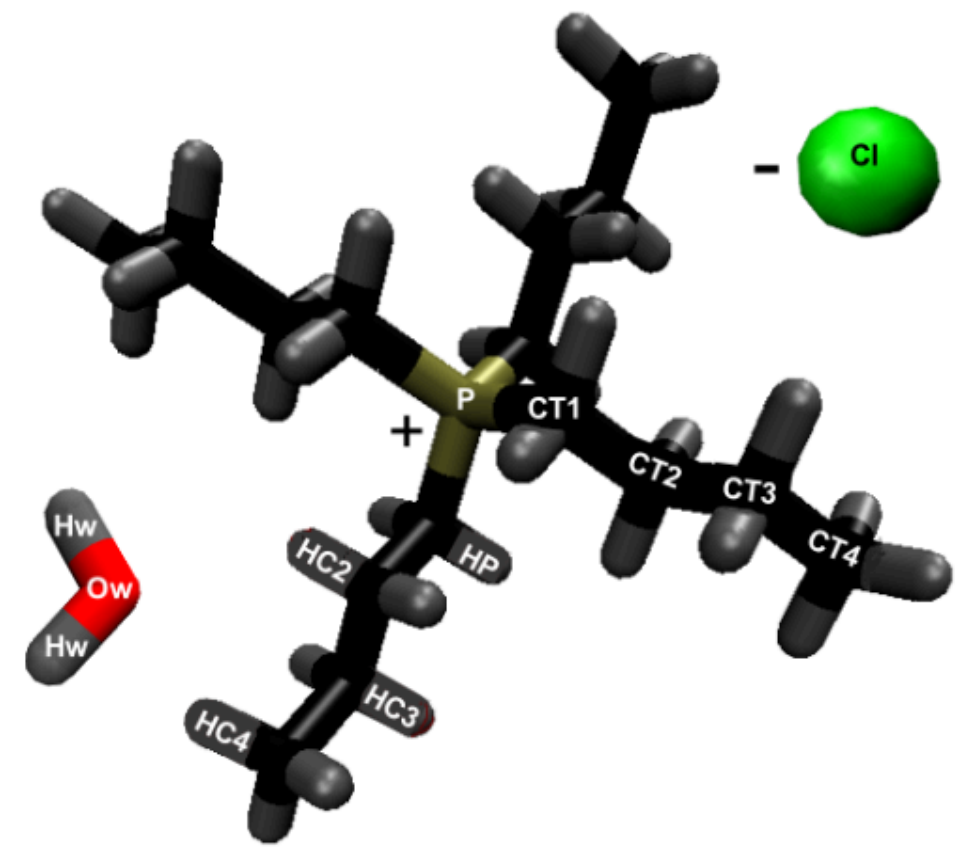

Figure (2.2) Atomic labeling for TBP, Cl, and water. The tan, black, red, and gray atoms are phosphorus, carbons, oxygens, hydrogens, respectively. (left) water molecule; (center) TBP molecule; (right) chloride molecule. For the TBP molecule, every atom was not labeled, but all butyl arms have the same symmetrical labeling. The right butyl arm is labeled with only the carbon atom labeling, while the bottom arm only has the hydrogen atoms labeled. The TBP atoms are labeled as follows: the CT's are the carbons; the HP's and HC's are the hydrogens; the $\mathrm{P}$ is the phosphorus. The water's atoms are the $\mathrm{Ow}$ and $\mathrm{Hw}$ for oxygen and hydrogens, respectively. The chloride is labeled as $\mathrm{Cl}^{22,30}$

The hydrogen bonding lifetime calculations show the average bonding time between the atoms, which represents the average strength of the hydrogen bonds. The MD Analysis H-bond autocorrelation package generated the hydrogen bonding lifetimes, which were calculated in a series of four separate simulations with different timesteps and run times. ${ }^{70-72}$ Four simulations with varying timesteps were utilized because it provided accurate hydrogen bonding lifetimes by minimizing the error in the time integration, especially in the case of the short hydrogen bonding lifetimes. All the additional simulations required to accurately calculate the hydrogen bonding lifetimes were started from the listed timepoints of the original simulations and stabilized for $0.2 \mathrm{~ns}$ before the data analysis began. The $10 \mathrm{ps}$ timesteps were taken from the original simulations. These four separate simulations were analyzed in the following order: simulation with a timestep of 0.01 ps and 100 ps run time; simulation with a timestep of $0.1 \mathrm{ps}$ and $1000 \mathrm{ps}$ run time; simulation with a timestep of 
1 ps and 10000 ps run time; finally the original simulation timestep of 10 ps with a run time that goes to the end of the simulation. The simulations were analyzed in order until one of the simulations provided a hydrogen bonding auto-correlation value of zero (the maximum value is 1). If a simulation finished without its auto-correlation value reaching zero, then the analysis continued by moving on to the next simulation in this series. Once a simulation in this series provided a zero value for the auto-correlation function, the current value of the hydrogen bonding lifetime was selected for the hydrogen bonding pair. If the auto-correlation function never reached zero even after the end of the last simulation in the series, the data were recorded, and the hydrogen bonding lifetime is presented with a "greater than" symbol $(>)$. All the presented hydrogen bonding lifetimes were averaged over 10 samples, with each sample using $80 \%$ of the runtime for its analysis.

\subsubsection{Simulations of the Small $I \beta$ Cellulose Bundle (18 Strands)}

After equilibration, the production runs were conducted using the NVT ensemble for the remaining $600 \mathrm{~ns}$. The smaller cellulose strands were simulated for a much longer time, as they required less computational power. Table 2.2 shows a detailed breakdown of the molecules in the simulation, and Figure 2.3 shows the initial configuration of the small cellulose bundle. The small cellulose bundle contains 12 glycans per strand. The simulations have final box dimensions of 99 to $105 \AA$, in all axis directions.

Table (2.2) Small cellulose bundle (18 Strands) simulation compositions ${ }^{22}$

\begin{tabular}{|c|c|c|c|c|c|c|}
\hline $\mathrm{Mol} \%$ & Total & Cellulose & $\begin{array}{c}\text { Total } \\
\text { Solvent }\end{array}$ & $\mathrm{Cl}$ & TBP & Water \\
\hline Water & Atoms & Atoms & Atoms & Molecules & Molecules & Molecules \\
\hline 63.1 & 95,058 & 4,590 & 90,468 & 1,530 & 1,530 & 2,616 \\
\hline 79.4 & 95,262 & 4,590 & 90,672 & 1,383 & 1,383 & 5,330 \\
\hline 86.8 & 95,199 & 4,590 & 90,609 & 1,229 & 1,229 & 8,081 \\
\hline 91.1 & 95,565 & 4,590 & 90,975 & 1,074 & 1,074 & 10,993 \\
\hline 93.9 & 96,054 & 4,590 & 91,464 & 913 & 913 & 14,054 \\
\hline 95.8 & 96,288 & 4,590 & 91,698 & 749 & 749 & 17,084 \\
\hline 100 & 108,507 & 4,590 & 103,917 & 0 & 0 & 34,639 \\
\hline
\end{tabular}




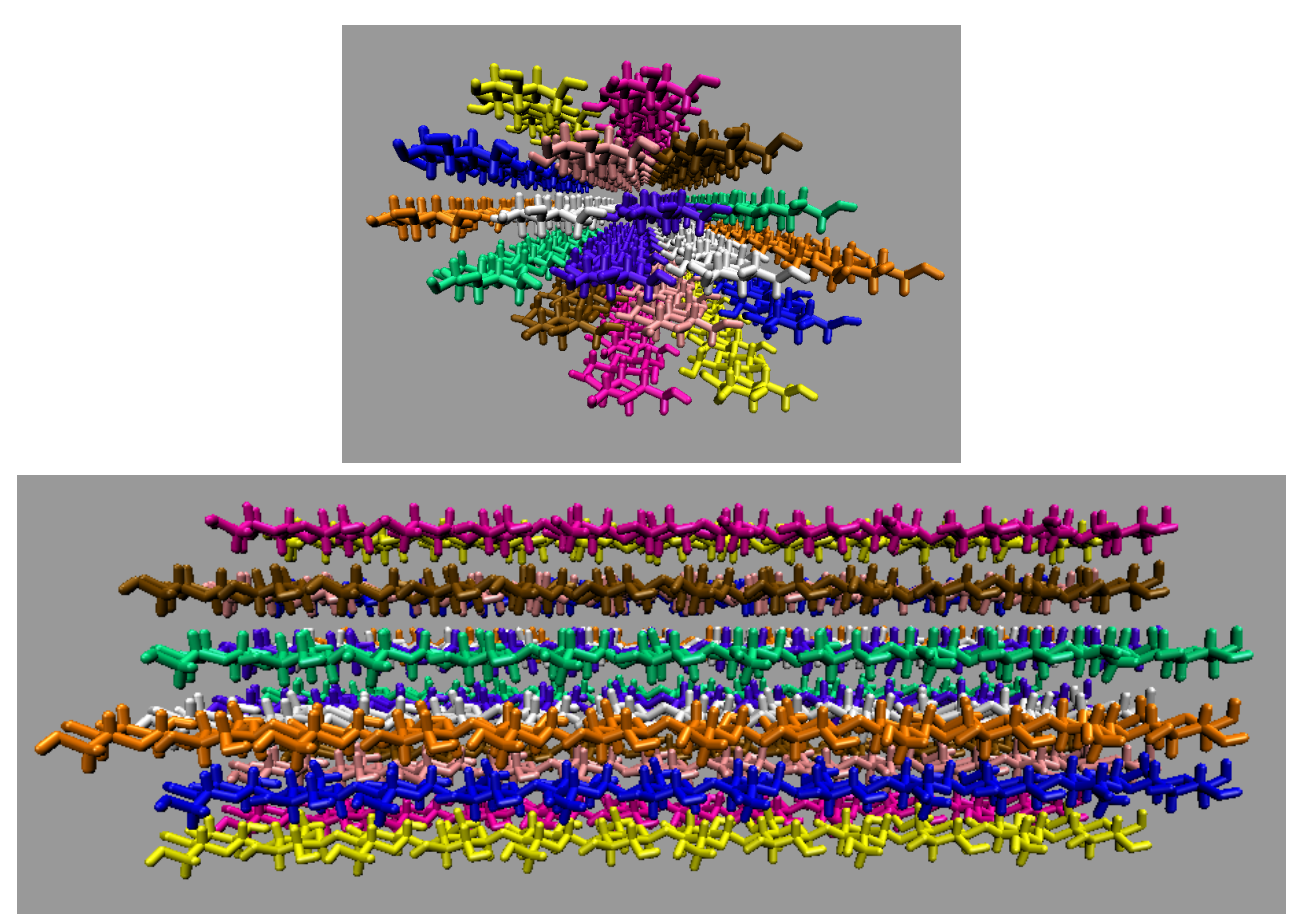

Figure (2.3) The initial configuration of the small $\mathrm{I} \beta$ cellulose bundle constructed via Cellulose-Builder. ${ }^{59}$ The cellulose bundle has 18 individual strands with 12 glycans units per strand. The mirror image cellulose strands and are identified using the same color. ${ }^{22,30}$

\subsubsection{Simulations of the Large $I \beta$ Cellulose Bundle (88 Strands)}

After equilibration, the production runs were conducted for the remaining $20 \mathrm{~ns}$ using the NVT ensemble. The duration of the simulations for the 88-strand bundle was short due to the computational cost of these large systems. Table 2.3 details the molecular breakdown of the simulations, and Figure 2.4 shows the initial configuration of the large cellulose bundle. The large cellulose bundle contains 24 glycans per strand. The simulations have final box dimensions of 185 to $191 \AA$, in all axis directions. 
Table (2.3) Large cellulose bundle (88 Strands) simulation compositions ${ }^{22}$

\begin{tabular}{|c|c|c|c|c|c|c|}
\hline Mol \% & Total & Cellulose & $\begin{array}{c}\text { Total } \\
\text { Solvent }\end{array}$ & $\mathrm{Cl}$ & TBP & Water \\
\hline Water & Atoms & Atoms & Atoms & Molecules & Molecules & Molecules \\
\hline 63.1 & 625,626 & 44,616 & 581,010 & 9,826 & 9,826 & 16,802 \\
\hline 79.4 & 621,438 & 44,616 & 576,822 & 8,798 & 8,798 & 33,910 \\
\hline 86.8 & 624,333 & 44,616 & 579,717 & 7,863 & 7,863 & 51,705 \\
\hline 91.1 & 618,765 & 44,616 & 574,149 & 6,778 & 6,778 & 69,379 \\
\hline 93.9 & 617,445 & 44,616 & 572,829 & 5,718 & 5,718 & 88,019 \\
\hline 95.8 & 614,886 & 44,616 & 570,270 & 4,658 & 4,658 & 106,246 \\
\hline 100 & 659,613 & 44,616 & 614,997 & 0 & 0 & 204,999 \\
\hline
\end{tabular}
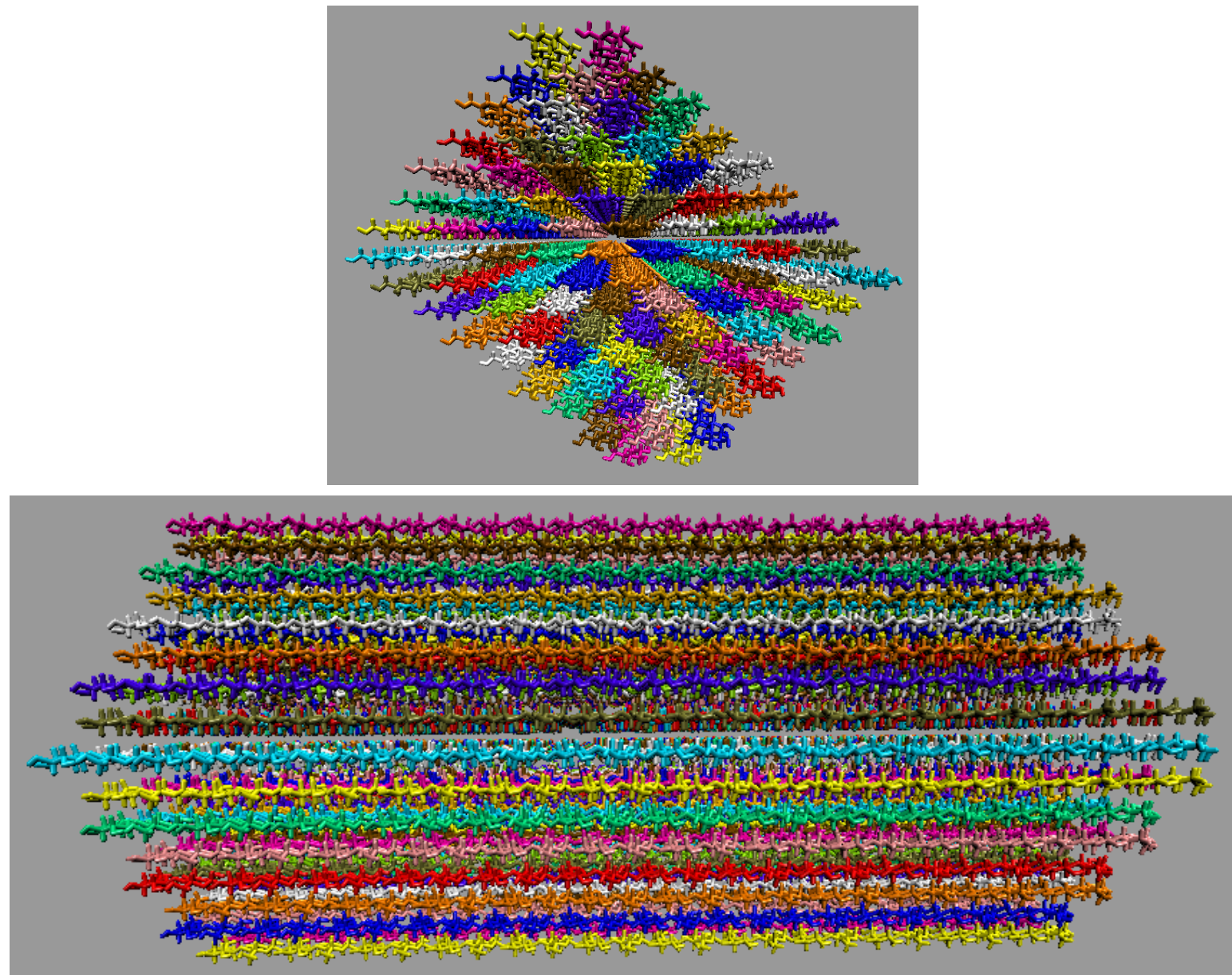

Figure (2.4) The initial configuration of the large $\mathrm{I} \beta$ cellulose bundle constructed via Cellulose-Builder. ${ }^{59}$ The cellulose bundle has 88 individual strands with 24 glycans units per strand. The mirror image cellulose strands and are identified using the same color. ${ }^{22,30}$ 


\section{Results}

\subsection{Simulation Validation}

\subsubsection{Force Field Validation: TBPH/TBPCl-Water Densities}

The simulated densities were calculated using the data from the TBPH-water and TBPClwater properties simulations via the NPT production runs. The force fields were validated using material safety data sheets (MSDS) for both the TBPH-water and TBPCl-water systems, as other scientific literature sources lacked this information. The tetrabutylammonium hydroxide (TBAH)-water and tetrabutylammonium chloride (TBACl)-water solutions were also utilized to supplement the densities, as the system is chemically similar to TBPH-water and TBPCl-water solutions, respectively. The TBPH-water, TBAH-water, and TBAClwater densities all confirm the viability of the selected force field parameters, as they are in good agreement with the known densities (see Figure 3.1).

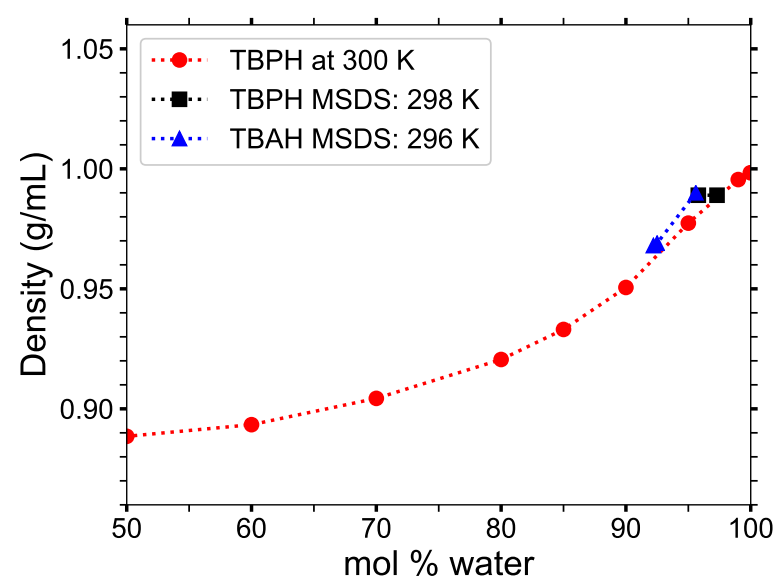

(a) TBPH density

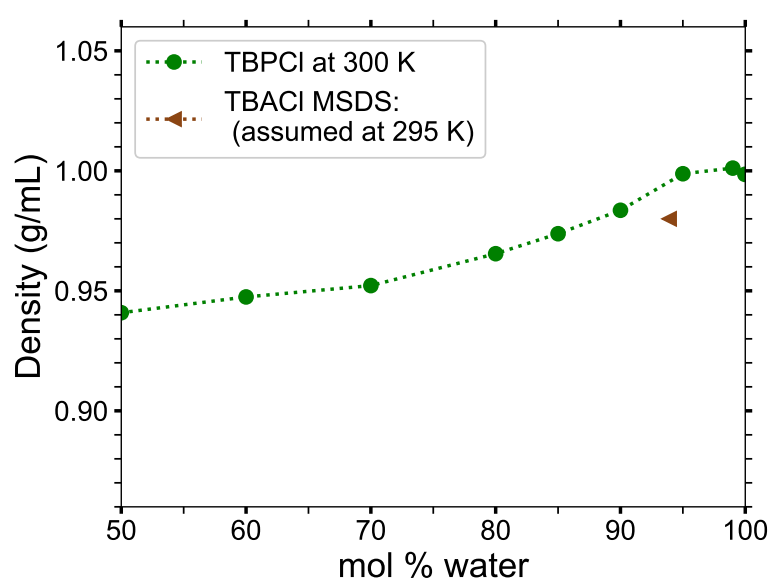

(b) TBPCl density

Figure (3.1) Simulated densities at $T=300 \mathrm{~K}$ and $p=1 \mathrm{~atm}$ compared with the MSDS values as a function of water concentration: ${ }^{58}$ (a) TBPH simulation densities compared to TBPH MSDS values at $95.8 \mathrm{~mol} \%$ water $(60 \mathrm{wt} \% \text { water })^{73,74}$ and TBAH MSDS values at $92.2 \mathrm{~mol} \%$ water $\left(45 \mathrm{wt} \%\right.$ water) ${ }^{75}$ and $95.6 \mathrm{~mol} \%$ water $\left(60 \mathrm{wt} \%\right.$ water) $;^{76}$ (b) $\mathrm{TBPCl}$ simulation density contrasted against the TBACl MSDS value at $93.9 \mathrm{~mol} \%$ water $(50 \mathrm{wt} \%$ water). ${ }^{77}$ 


\subsubsection{Small and Large I $\beta$ Cellulose Bundle Comparison in TBPCl-water}

The stability of the cellulose bundle is dependent on the intra-strand (within a strand) and inter-strand (between strands) hydrogen bonding network. ${ }^{8,15,78}$ Recent studies have shown that breaking the intra-strand hydrogen bonds is the critical step in the cellulose dissolution process. ${ }^{8,15,78}$ Rabideau et al. showed that while the inter-strand hydrogen bonds are quickly broken in a cellulose strand, the cellulose strand does not peel away from the bundle until the intra-strand hydrogen bonds are broken. ${ }^{15}$ Therefore, breaking the intra-strand hydrogen bonds appears to be the upper threshold to cellulose dissolution. ${ }^{15}$ In this study, the critical intra-strand hydrogen bonding was the same between the solventexposed layers (i.e., outer layers or the first and second layers, corner strands, and the strands above the corner strands) of the small and large bundles, while there were significant differences for the inter-strand hydrogen bonding (see Figures 3.3, 7.1, 7.2, 7.3, and 7.4). This difference in the inter-strand hydrogen bonding could be due to the additional twisting of the small cellulose bundle, as shown by Hadden using the GLYCAM06 force field et al. ${ }^{25}$ Hadden et al. also showed that the number of strands in a cellulose bundle stabilizes it from twisting more than the number of glycans per strand, at least for strands with 20 glycans or more in a water solution. ${ }^{25}$ Estimating the twisting in these simulations with the data from Hadden et al. yields an approximated $6 \%$ and $1 \%$ twisting of the small and large bundles, respectively. The experimental cellulose twisting provided by Hanley et al. was approximately $0.25 \%$ for a 720 strand bundle. ${ }^{25,79}$ Rabideau et al. showed the intra-strand hydrogen bonding is critical to cellulose dissolution using a much smaller cellulose bundle (a 10 strand I $\beta$ bundle with 8 glycans per strand). ${ }^{15}$ The present study compares the TBPClwater (solvent) hydrogen bonding between the small and large cellulose bundles, which show the same hydrogen bonding between the small and large cellulose bundles (see Figures 3.4, $3.5,7.5,7.6,7.7,7.8,7.9$, and 7.10). The small cellulose bundle size was increased from that of past simulations, which used ILs to study cellulose dissolution. ${ }^{15}$ Since the large-bundle simulations are computationally infeasible for long simulation time, and the small bundle simulations have the same intra-strand and solvent hydrogen bonding for the solvent-exposed cellulose strands, the 6-fold reduction in the computation cost of the simulations is justified. 
Figures 3.2 and 3.3 show the averaged intra-strand and inter-strand hydrogen bonds per glycan, which normalized the data between the small and large bundles for the comparison across a range of water concentrations. The data were averaged from 10 to $20 \mathrm{~ns}$, since only the center strands in the small bundle were not stabilized after $10 \mathrm{~ns}$. The inter-strand hydrogen bonding of the center strands was stabilized in both the small and large bundles within $10 \mathrm{~ns}$. The intra-strand hydrogen bonding of the center strands within the large bundle was stabilized within $3 \mathrm{~ns}$, but the center strands of the small bundles did not stabilize even after $20 \mathrm{~ns}$. Therefore, the intra-strand hydrogen bonding of the center strands in the small cellulose bundle should stabilize at a lower value than listed in Figures 3.3b and 7.1b.

The intra-strand hydrogen bonding was approximately the same between the small and large bundles at all the concentrations, except for the center strands (see Figures 3.2a, 3.2b, 3.3a, 3.3b, 7.1, and 7.2). The solvent-exposed strands had approximately the same intrastrand hydrogen bonding between the small and large bundles, due to the lack of stabilization from hydrogen bonding between neighboring strands (i.e., the absence of neighboring strands on the solvent-exposed side). The center strands of the large bundles are stabilized better by their surrounding strands since the overall number of hydrogen bonds is higher, and the strands are further away from the solvent. Both of these items increase the structural support of the large bundle with increasing bundle thickness. At the same time, the large bundle also does not appear to be as twisted as the small bundle. The intra-strand hydrogen bonding of the center strands is starkly different between the small and large cellulose bundles, with the large bundle retaining nearly $85 \%$ of its hydrogen bonds (approximately 2 per glycan is perfect) and coming to equilibrium in $3 \mathrm{~ns}$, while the small bundle has not come to equilibrium and only retained approximately $60 \%$ of its hydrogen bonds at $20 \mathrm{~ns}$. Overall, the cellulose bundle's first or second layers that dissolve in the simulations will have the same intra-strand hydrogen bonding for both the small and large cellulose bundles.

The inter-strand hydrogen bonding is significantly different between the small and large cellulose bundles, except at $63.1 \mathrm{~mol} \%$ water (see Figures 3.2a, 3.2b, 3.3c, 3.3d, 7.3, and 7.4). However, at $63.1 \mathrm{~mol} \%$ water the inter-strand hydrogen bonds are drastic outliers from the rest of the concentrations, with all the values between the small and large bundles being nearly identical. These outliers suggest that lower water concentrations may produce very 
similar results between the small and large cellulose bundles. In this work, the difference in the cellulose twisting was not quantified between the differing TBPCl-water concentrations. However, the small cellulose bundle does visually appear more twisted than the large cellulose bundle for all the concentrations (see Figures 3.2a and 3.2b). In agreement with these data, the cellulose structure twisting is well documented for the GLYCAM06 force field in pure water and under vacuum, ${ }^{24}$ being more pronounced for small cellulose bundles and under vacuum conditions. ${ }^{25,26,79}$

The number of hydrogen bonds between the TBPCl-water solvent and the cellulose bundle is also an important point of comparison. The hydrogen bonds were compared between the Cl, TBP, and water to the strands for both the small and large cellulose bundles. This comparison shows that the solvent-strand hydrogen bonding for the small and large cellulose bundles was nearly the same (see Figures 3.4 and 3.5). Additionally, the standard deviations for the solvent-strand hydrogen bonding can be found in Figures 7.5, 7.6, 7.7, 7.8, 7.9, and 7.10. Since the first layer and corner strands are more solvent-exposed and have the most solvent-strand hydrogen bonds (i.e., interaction with the solvent), they should have the highest probability of strand separation and dissolution.

Using a small cellulose bundle, the GLYCAM06 force field may be capable of identically simulating the outer strand's hydrogen bonds of a full-scale cellulose bundle for low water concentration ILs. ${ }^{24}$ However, the replication of identical simulations is likely dependent on the attractive Van der Waals (VDW) interactions of the individual IL-water solution, as pointed out by Hadden et al. in a pure water solution. ${ }^{25}$ Alternatively, this could mean that even larger cellulose bundles are required to simulate some low water concentration ILs or this $63.1 \mathrm{~mol} \%$ water with the large cellulose bundle simulation is a statistical outlier. It is also important to point out that the vacuum space surrounding the cellulose (i.e., the void space or absence of very close molecules around the cellulose due to the deletion of the solvent in the area) increased at the start of these simulations, as the water concentration decreased. This vacuum space increase was unavoidable due to more TBP molecules in the solution and deleting the solvent to insert the cellulose. Also, due to the shape of TBP, the molecular void spaces between the TBP arms increase with decreasing water concentrations, creating potential vacuum spaces or attractive VDW interaction disruptions. ${ }^{58}$ Further study of the 
GLYCAM06 force field is required to confirm that the smaller and larger cellulose bundles outer strands produce similar results for ILs at low water concentrations. ${ }^{24}$

This study suggests that the GLYCAM06 force field is capable of adequately simulating the solvent-exposed layers of a large cellulose bundle by only using a small cellulose bundle, at least in the TBPCl-water solution at these water concentrations. ${ }^{24,26}$ The data show that the intra-strand hydrogen bonding was the same between the solvent-exposed cellulose strands, while the inter-strand hydrogen bonding was different between the small and large cellulose bundles. Specifically, the small bundles are a reasonable substitute for the large bundles, because the breaking of the intra-strand hydrogen bonds is the critical step in the cellulose dissolution process. ${ }^{8,15,78}$ The TBPCl-water (solvent)-strand hydrogen bonding between the small and large cellulose bundles also produced nearly identical results. These results may also apply to other IL-water or IL co-solvent combinations, but more research is required to make a definitive conclusion. It is currently not computationally feasible to simulate the large cellulose bundles in the TBPCl-water solution at various concentrations beyond 100 ns, let alone 600 ns. For the TBPCl-water concentrations studied in this paper, most of the critical system dynamics occurs after $100 \mathrm{~ns}$, so simulating the large cellulose bundles even up to $100 \mathrm{~ns}$ would provide minimally useful data. Since all the hydrogen bonding between the small and large cellulose bundles is nearly identical, except for the center strands and the inter-strand hydrogen bonding, the six-fold reduction in the computational cost, which make the simulations computationally possible is justified. A continued hydrogen bonding evolution throughout these simulations is expected, as the GLYCAM06 force field is slowly changing over time and never fully stabilizing in a water solution even after $800 \mathrm{~ns}$ or longer. ${ }^{24,26}$ 


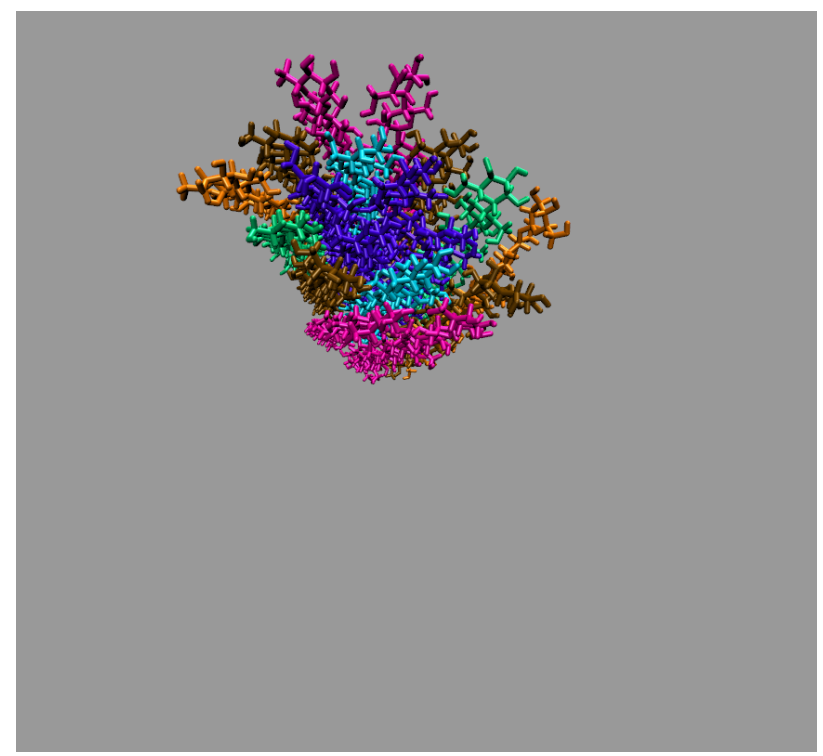

(a) Small bundle coloring scheme: $63.1 \mathrm{~mol} \%$ water at $10 \mathrm{~ns}$

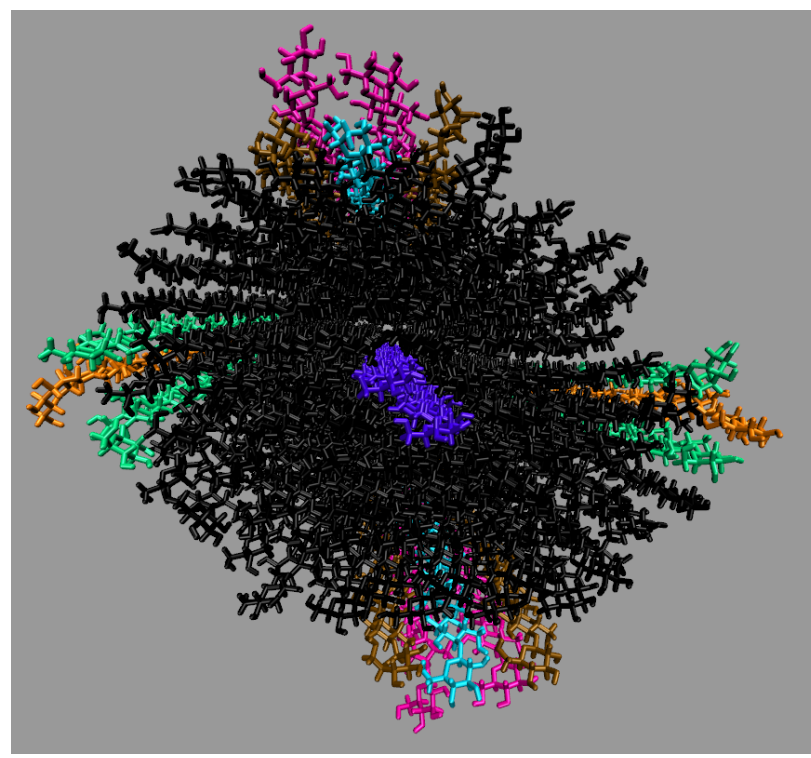

(b) Large bundle coloring scheme: $63.1 \mathrm{~mol} \%$ water at $10 \mathrm{~ns}$

Figure (3.2) Coloring scheme for the hydrogen bonding comparison between the small and large cellulose bundles: (a) small bundle coloring scheme; (b) large bundle coloring scheme. The colored strands are matched and compared between the plots for the small and large bundles and the images in this figure to identify similar hydrogen bonding behavior based on their locations (see Figures 3.3). The black cellulose strands are not compared. ${ }^{22}$ 


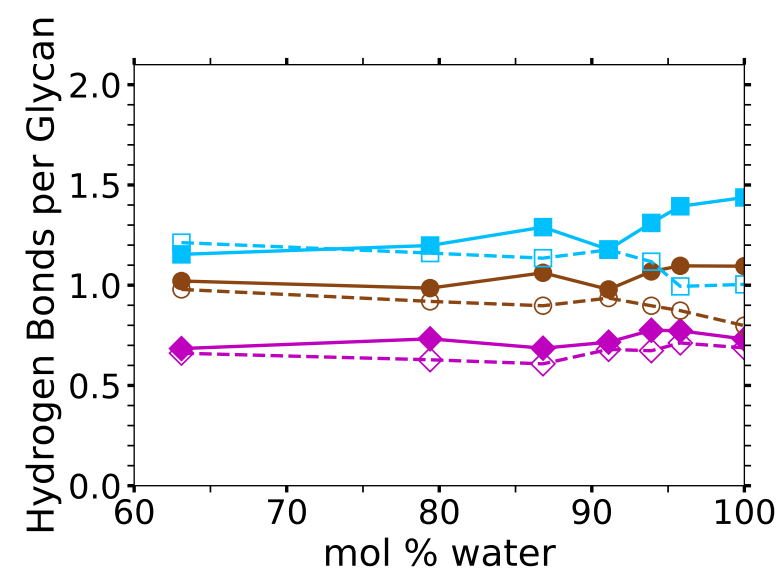

(a) Intra-strand hydrogen bonds: first (dark pink) and second layer strands (brown and light blue)

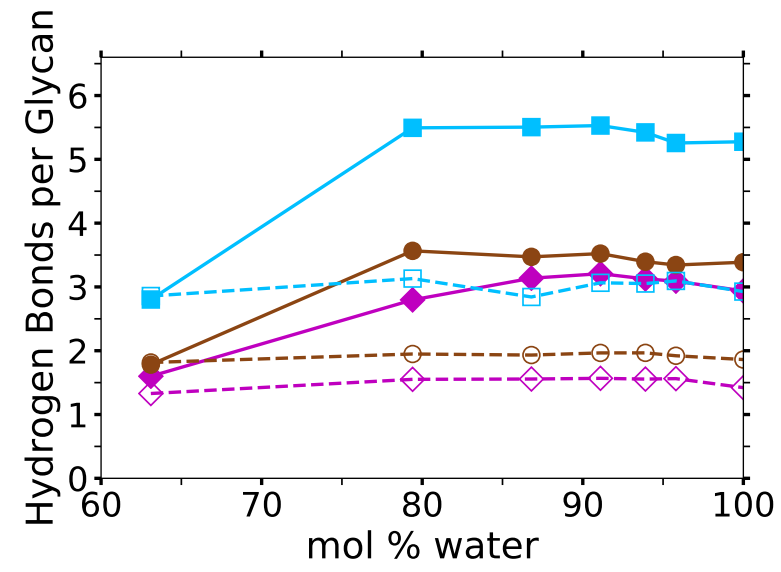

(c) Inter-strand hydrogen bonds: first (dark pink) and second layer strands (brown and light blue)

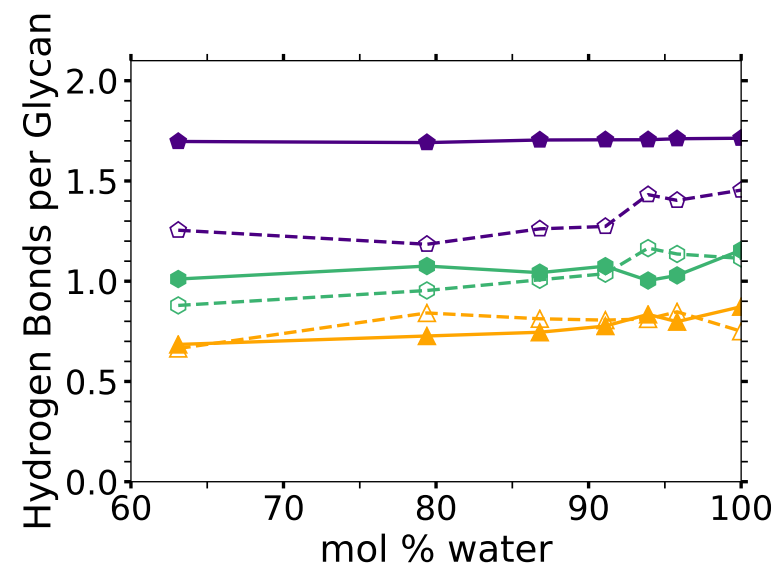

(b) Intra-strand hydrogen bonds: center (purple), corner (orange), and the strands above the corner strands (green)

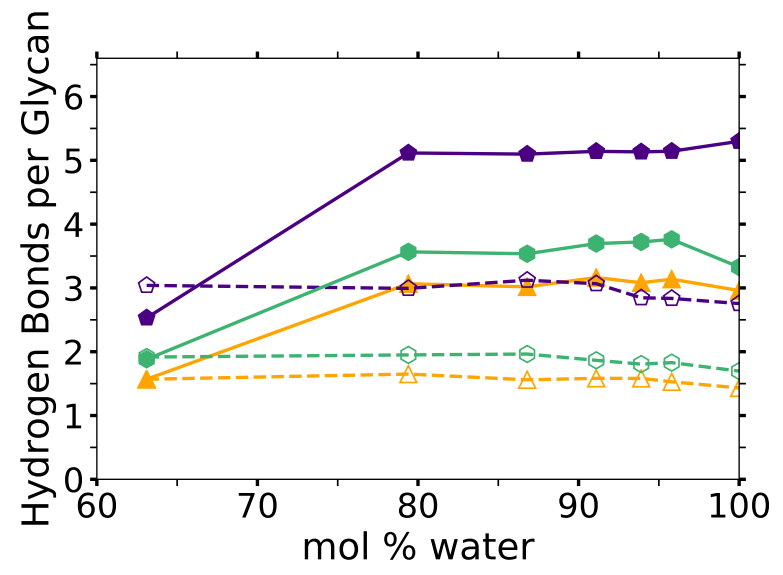

(d) Inter-strand hydrogen bonds: center (purple), corner (orange), and the strands above the corner strands (green)

Figure (3.3) Average inter-stand and intra-strand hydrogen bonding comparison between the small and large cellulose bundles at various water concentrations: (a) the intra-strand hydrogen bonds for the first and second layers; (b) the intra-strand hydrogen bonds for the center, corner, and the strands above the corner strands; (c) the inter-strand hydrogen bonds for the first and second layers; (d) the inter-strand hydrogen bonds for the center, corner, and the strands above the corner strands. All data points were averaged from 10 to 20 ns. The solid lines and filled markers represent the large cellulose bundle, and the dashed lines and unfilled markers show the small cellulose bundle. The colored strands are matched and compared between the plots for the small and large bundles and the images to identify similar hydrogen bonding behavior based on their locations (see Figures 3.2a and 3.2b for the color-coded images). The black cellulose strands are not compared. The small and large cellulose bundle intra-strand hydrogen bonding is based on 11 glycans and 23 glycans, as the last glycan has no potential bonding partner. ${ }^{22}$ 


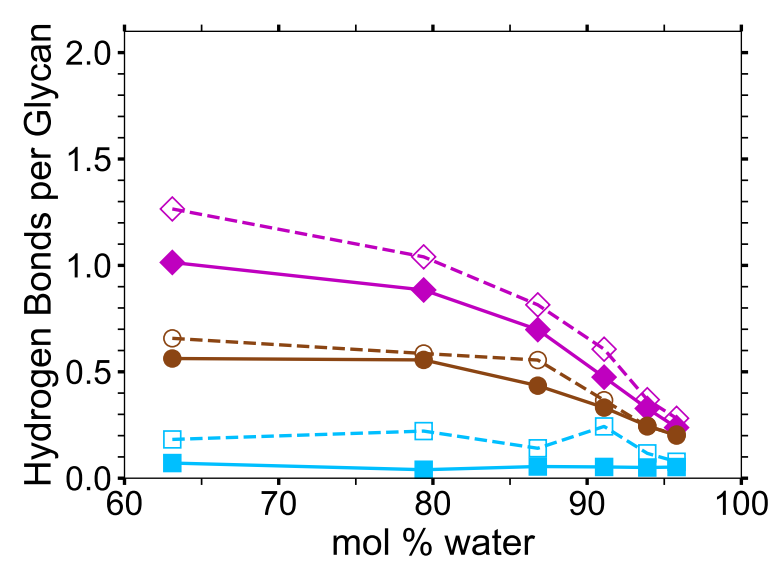

(a) Cl-strand hydrogen bonds: first (dark pink) and second layer strands (brown and light blue)

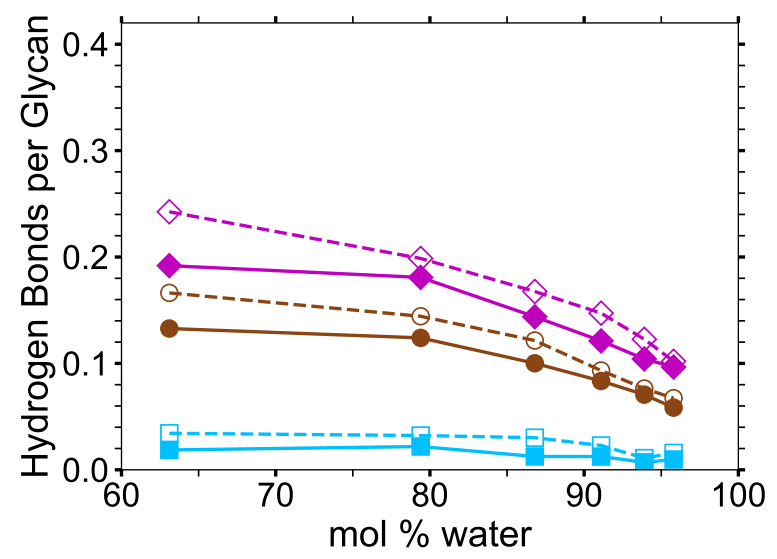

(c) TBP-strand hydrogen bonds: first (dark pink) and second layer strands (brown and light blue)

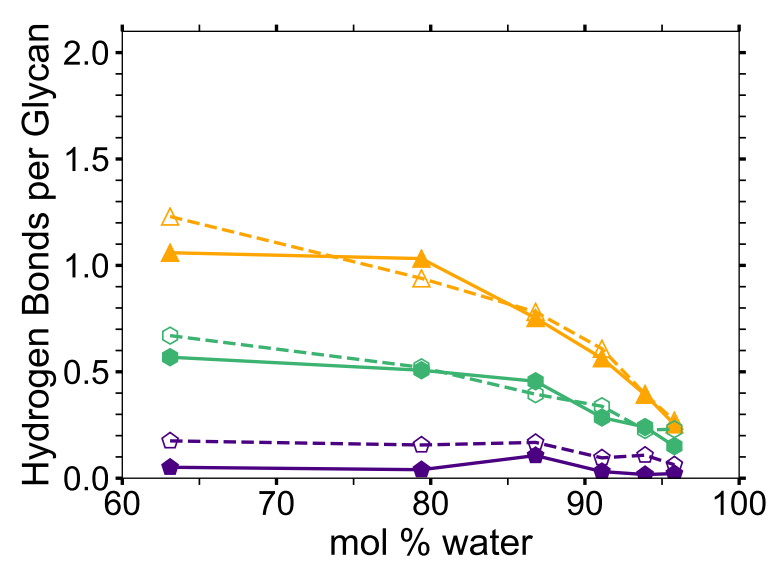

(b) Cl-strand hydrogen bonds: center (purple), corner (orange), and the strands above the corner strands (green)

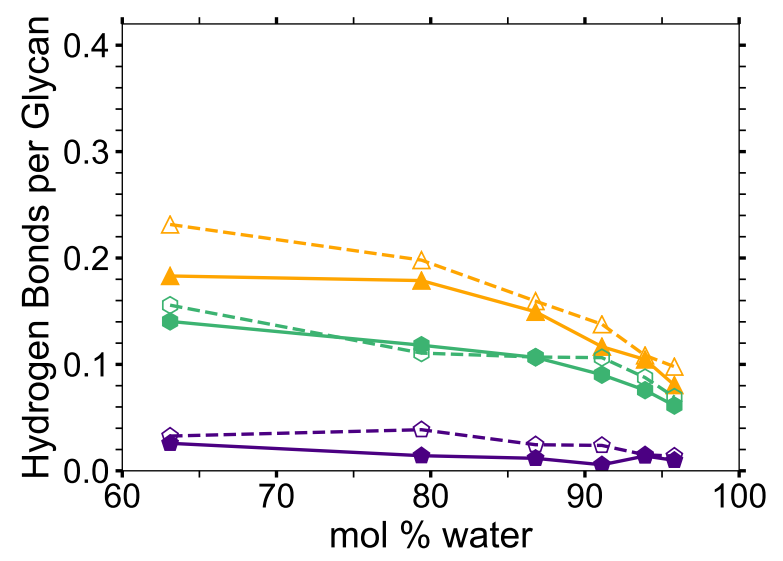

(d) TBP-strand hydrogen bonds: center (purple), corner (orange), and the strands above the corner strands (green)

Figure (3.4) Average solvent hydrogen bonding comparison between the small and large cellulose bundles at various water concentrations (Part 1 of 2): (a) the Cl-strand hydrogen bonds for the first and second layers; (b) the Cl-strand hydrogen bonds for the center, corner, and the strands above the corner strands; (c) the TBP-strand hydrogen bonds for the first and second layers; (d) the TBP-strand hydrogen bonds for the center, corner, and the strands above the corner strands. All data points were averaged from 10 to $20 \mathrm{~ns}$. The solid lines and filled markers represent the large cellulose bundle, and the dashed lines and unfilled markers show the small cellulose bundle. The colored strands are matched and compared between the plots for the small and large bundles and the images to identify similar hydrogen bonding behavior based on their locations (see Figures 3.2a and 3.2b for the color-coded images). The black cellulose strands are not compared. ${ }^{22}$ 


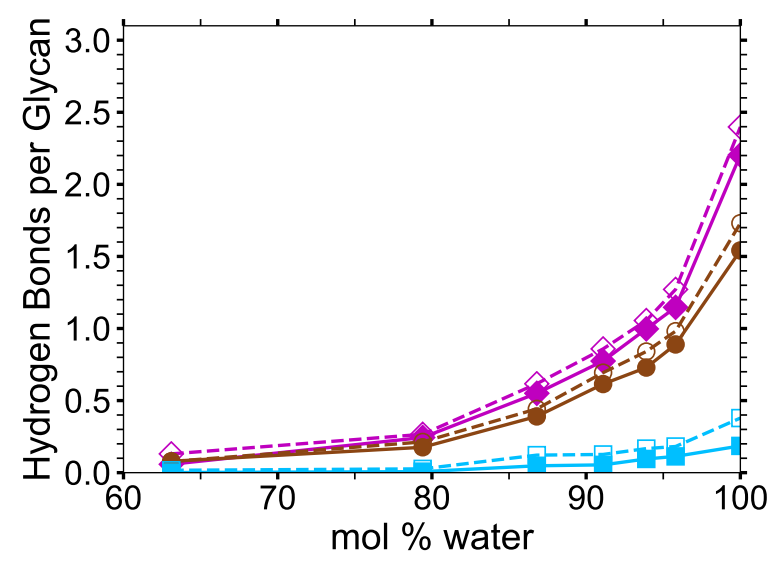

(a) Water-strand hydrogen bonds: first (dark pink) and second layer strands (brown and light blue)

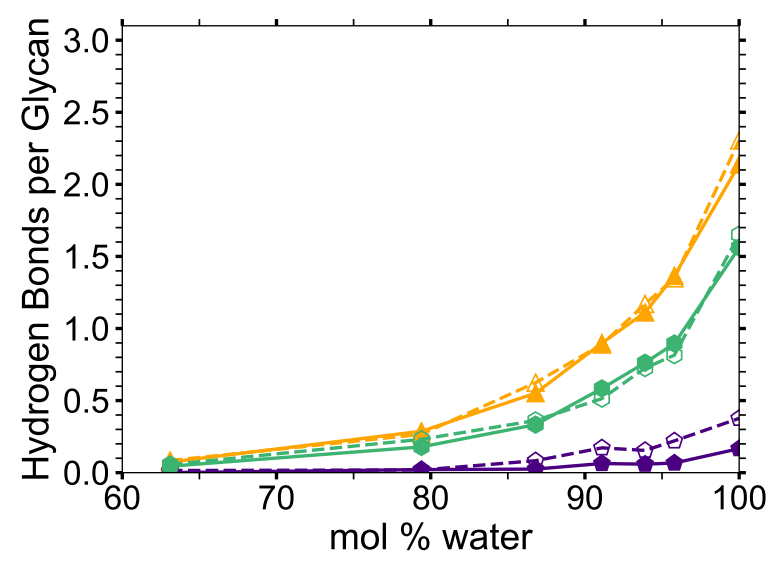

(b) Water-strand hydrogen bonds: center (purple), corner (orange), and the strands above the corner strands (green)

Figure (3.5) Average solvent hydrogen bonding comparison between the small and large cellulose bundles at various water concentrations (Part 2 of 2): (a) the water-strand hydrogen bonds for the first and second layers; (b) the water-strand hydrogen bonds for the center, corner, and the strands above the corner strands. All data points were averaged from 10 to 20 ns. The solid lines and filled markers represent the large cellulose bundle, and the dashed lines and unfilled markers show the small cellulose bundle. The colored strands are matched and compared between the plots for the small and large bundles and the images to identify similar hydrogen bonding behavior based on their locations (see Figures 3.2a and 3.2b for the color-coded images). The black cellulose strands are not compared. ${ }^{22}$ 


\subsection{Structural Properties}

\subsubsection{Radial Distribution Functions (RDFs) for the TBPCl-Water Solution}

The radial distribution functions (RDFs) were all determined using an atom that represented an approximate center of mass of a given molecule, except for the $\mathrm{Cl}^{-}$anion which is the exact center of mass. The $\mathrm{TBP}^{+}$cation used the phosphorus atom (P atom) as its approximate center of mass. Both the water and hydroxide anion $\left(\mathrm{OH}^{-}\right)$utilized the oxygen atom $(\mathrm{O}$ atom) as their approximate center of mass. These approximate centers of mass were also used for the anomalous diffusion calculations. Figures 3.6 and 3.7 show the RDF data for the TBPCl-water solutions. Due to the nearly identical RDFs of the TBPH-water solutions, the TBPH-water solutions plots are not in the main body of the document but attached at the end of this document in the Appendix (see Figures 7.11, 7.12, and 7.13). The RDF curves were calculated at $300 \mathrm{~K}$ with the data plotted as a smoothed curve using a rolling average. Plotting the rolling averages reduced the data scatter, allowing the RDFs to be compared at various concentrations.

Figure 3.6a shows the $\mathrm{Cl}^{-}-\mathrm{Cl}^{-}$RDFs. For the first solvation shell there is a sharp peak around $4.6 \AA$ at $50 \mathrm{~mol} \%$ water, which almost doubles in size when the solution is diluted to $80 \mathrm{~mol} \%$ water. The chlorides are moving toward each other, also indicating that these chloride anions are being drawn into the water pocket between the TBP arms and away from the $\mathrm{TBP}^{+}$phosphorous atom. The driving force for the reduced $\mathrm{Cl}^{-}$to $\mathrm{Cl}^{-}$distances is the solvation of these anions with water. After the water fully solvates the $\mathrm{Cl}^{-}$anion (95 to $99 \mathrm{~mol} \%$ water) and with water dilution being a contributing factor, this RDF peak is no longer dominant (also see Figure 3.25b).

Between 85 and $95 \mathrm{~mol} \%$ water, the $\mathrm{TBP}^{+}-\mathrm{TBP}^{+}$RDFs are mostly stable, which is intriguing since this is the approximate region where cellulose solubility maxima occur in the TBPH-water solution, and where the cellulose solubility is rapidly declining in the TBPClDMF solution (see Figure 3.6b). The RDF data imply that the TBPs centers remain at a consistently stable distance within this range of water concentrations. Further water dilution is likely responsible for the disruption of this stable distance at $95 \mathrm{~mol} \%$ water or higher, which is alternately described later in the $\mathrm{TBP}^{+}$clustering data. From 50 to $99 \mathrm{~mol} \%$ water 


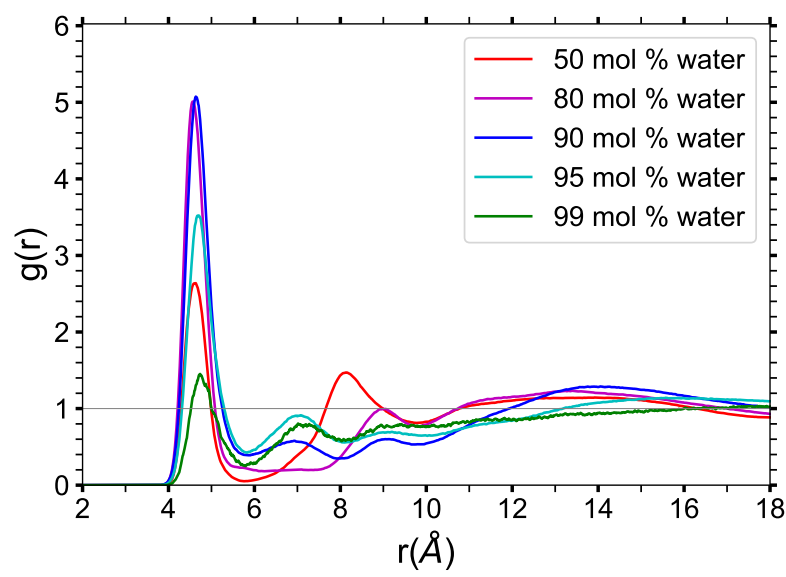

(a) $\mathrm{Cl}^{-}-\mathrm{Cl}^{-}$

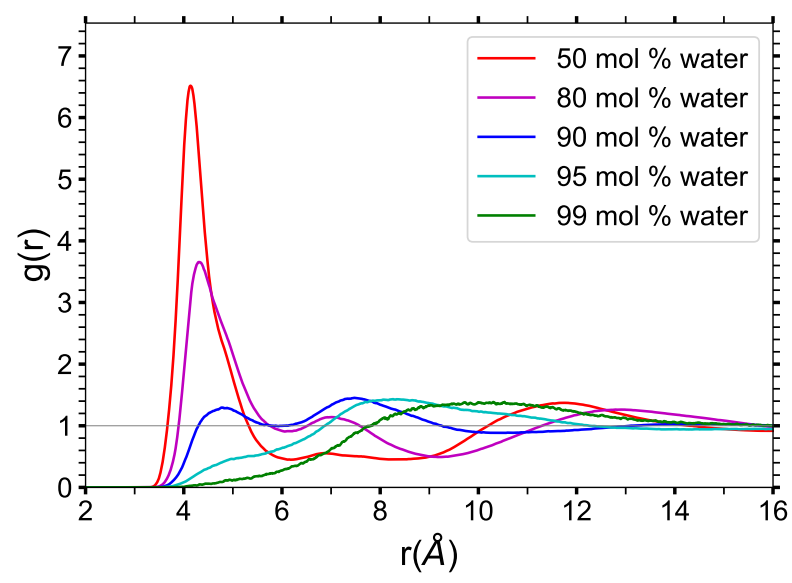

(c) $\mathrm{TBP}^{+}-\mathrm{Cl}^{-}$

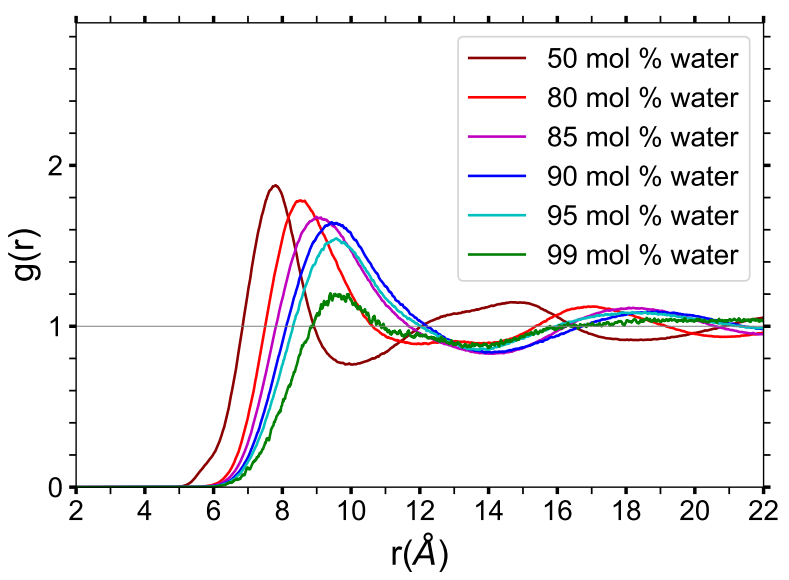

(b) $\mathrm{TBP}^{+}-\mathrm{TBP}^{+}$

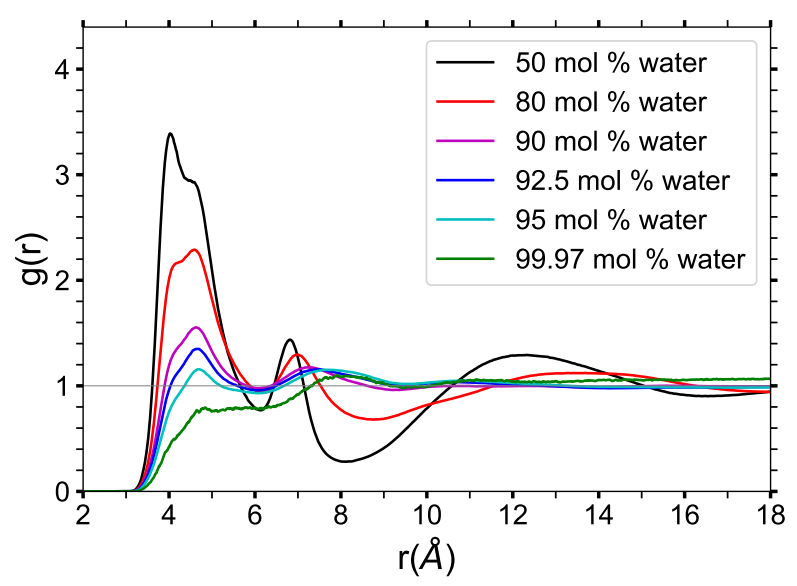

(d) $\mathrm{TBP}^{+}-\mathrm{H}_{2} \mathrm{O}$

Figure (3.6) Radial distribution functions of TBPCl-water at $300 \mathrm{~K}$ over a range of water concentrations (Part 1 of 2$).{ }^{58}$ 


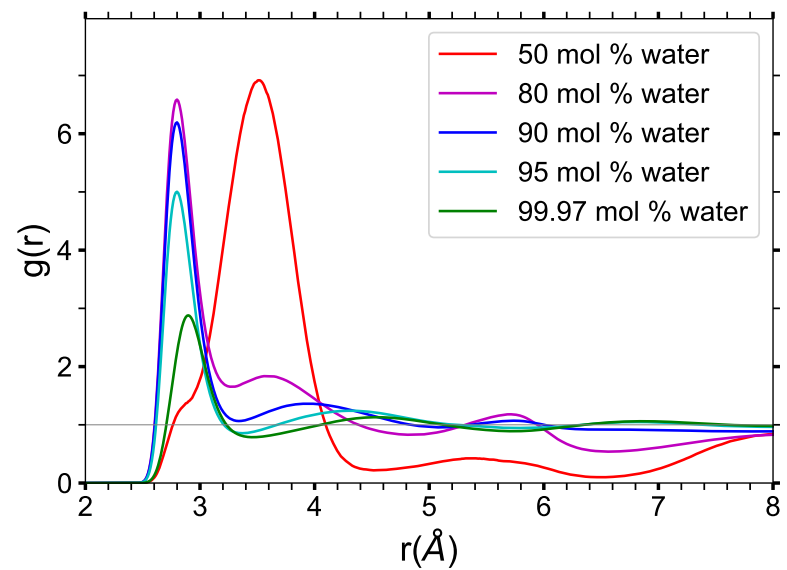

(a) $\mathrm{H}_{2} \mathrm{O}-\mathrm{H}_{2} \mathrm{O}$

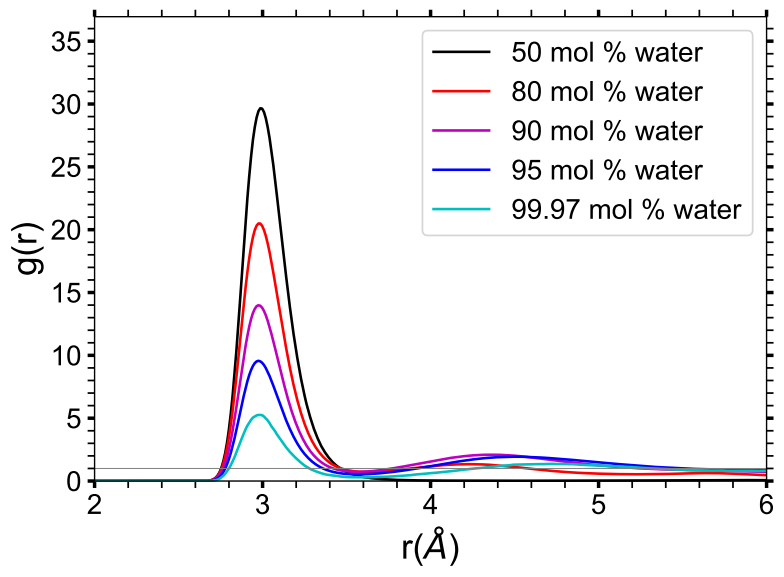

(b) $\mathrm{H}_{2} \mathrm{O}-\mathrm{Cl}^{-}$

Figure (3.7) Radial distribution functions of TBPCl-water at $300 \mathrm{~K}$ over a range of water concentrations (Part 2 of 2$).{ }^{58}$

the TBP to TBP distance is continually growing, which is in-line with the simulation's visual observations and calculations of the TBP structure distinctly changing in this range of water concentrations (also see Figures 3.9b, 3.10, 3.12, and 3.13).

Supporting the evidence that the $\mathrm{Cl}^{-}$anions are being pulled into the nearby water pocket areas between the TBP arms via hydrogen-bonding interactions with water, the $\mathrm{TBP}^{+}-\mathrm{Cl}^{-}$ RDFs shows the separation of the TBP and $\mathrm{Cl}$ ions with increasing water concentration (see Figure 3.6c). The RDFs directly show this, as the first peak in the $50 \mathrm{~mol} \%$ water data at around $4.1 \AA$ is drastically reduced in magnitude and stretched out to $4.3 \AA$ by $80 \mathrm{~mol} \%$ water. Once the system is very diluted by water (95 to $99 \mathrm{~mol} \%$ water), there is only a singular RDF peak of a small magnitude with the RDF distances stretched to approximately 8 to $10 \AA$, respectively (also see Figures 3.12 and 3.13).

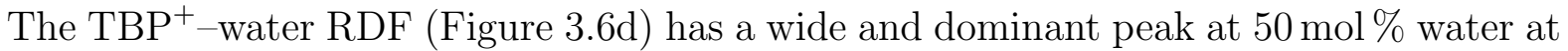
around $4 \AA$. This wide peak implies the water molecules have some freedom to move around and do not form a unique structure near the center of the TBP (phosphorous atom), due to the void space or shifting water clusters between the TBP's butyl arms (also see Figures 3.12, and 3.13). At $80 \mathrm{~mol} \%$ water, the dominant peak is at approximately $4.5 \AA$ indicating the loss of the larger $\mathrm{Cl}$ anion near the phosphorous atom, as the $\mathrm{Cl}$ anion has started to move into the growing water pocket between the TBP's butyl arms. Some structural patterns are starting to emerge in the solution as observed from the formation of the second and third 
RDF peaks. The addition of these structural patterns is believed to develop from the ordered structure of the chloride-water hydrogen bonding, with the Coulombic forces allowing the chloride and the phosphorus atoms to maintain close proximity. After the concentration exceeds $85 \mathrm{~mol} \%$ water, the TBP's butyl arms and clusters are broken, further shifting the $\mathrm{TBP}^{+}{ }_{-}$water $\mathrm{RDF}$ peaks to a larger distance; however, some of this shift can also be attributed to water dilution (also see Figures $3.9 \mathrm{~b}$ and 3.10 ).

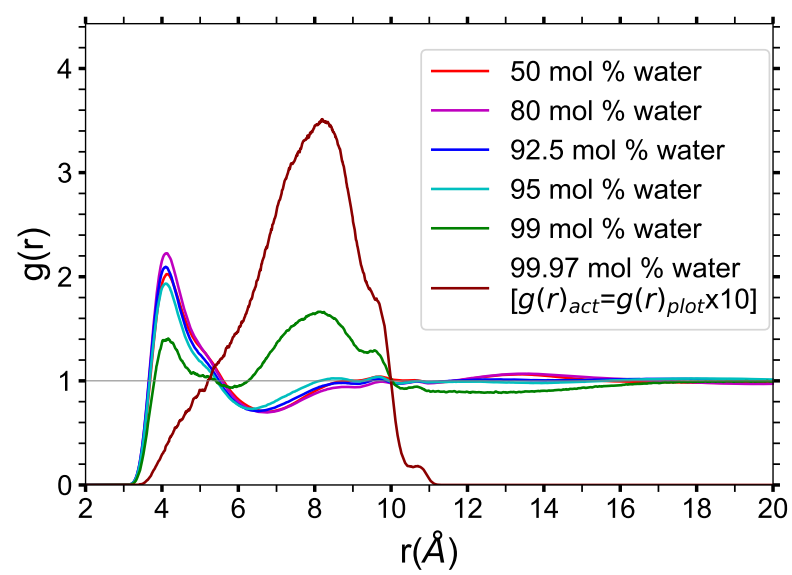

(a)

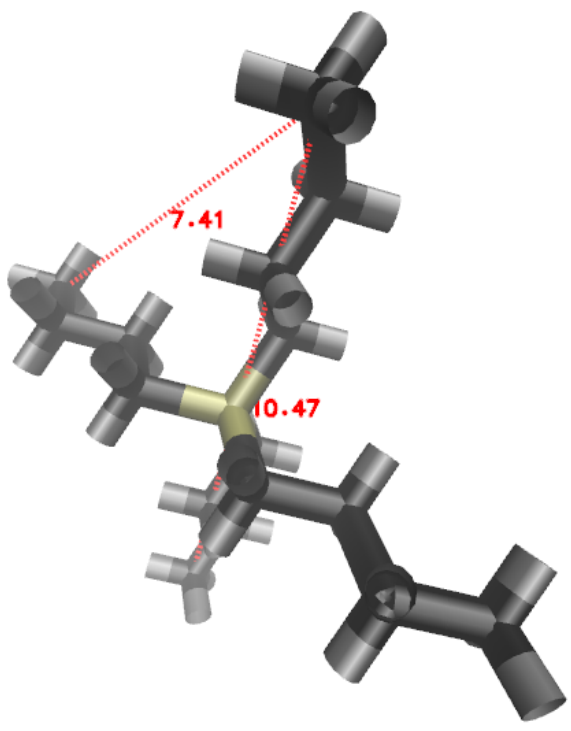

(b)

Figure (3.8) (a) The RDFs between the end carbons (CT4-CT4) of TBP's butyl arms in the TBPCl-water solution at $300 \mathrm{~K}$. Note that the $99.97 \mathrm{~mol} \%$ water data is an order of magnitude higher than the other data since it was scaled down by $1 / 10$ to fit on the same plot; (b) Radial distance between the end carbons of the TBP's butyl chains (CT4's), on an individual molecule. ${ }^{30,58}$

The end carbons on the TBP arms (CT4's) are analyzed using RDFs, to further investigate the unique structure which TBP forms throughout the cellulose solubility region (see Figure 3.8). When TBP is infinitely diluted in water $(99.97 \mathrm{~mol} \%)$, these end carbons display an RDF with a maximum of around $8.2 \AA$, characteristic of the average distance of the two closest neighboring arms. While this same trend is noticeable in the RDF data at $95 \mathrm{~mol} \%$, it is an order of magnitude smaller. The dominant peaks at approximately $4.1 \AA$ are mostly attributed to $C T 4-C T 4$ distance from a TBP molecule and its neighboring TBP molecule.

The CT4-CT4 RDF data mostly maintain a constant value for the TBPCl-water solutions 
of less than $95 \mathrm{~mol} \%$ water, which is visualized in Figure $3.8 \mathrm{a}$ at approximately $4.1 \AA$ and $8 \AA$. This distance between the $C T 4$ carbons of a TBP molecule and its neighbor does not begin to decrease in magnitude until the water concentration is between 95 to 99 mol\%, which is the region where cellulose is no longer soluble in the either the TBPH-water or the TBPCl-DMF solutions. Therefore, the RDF CT4-CT4 distances are more or less stable in and below the cellulose solubility region of 79.4 to 93.9 mol \% water. ${ }^{16}$ However, the constant value does not tell the whole story since the $C T 4$ may initially be closer to the inside of the neighboring TBP molecule (i.e., neighboring phosphorous atom) at $50 \mathrm{~mol} \%$ and move to the outside of the neighboring TBP molecule at a higher water concentrations (also see Figures $3.9 \mathrm{~b}, 3.10)$. There is also a noticeable shift upward in the RDF at $8.5 \AA$ for the $95 \mathrm{~mol} \%$ water concentration, as the TBP cation loses its neighbors due to the larger globular water structure beginning to surround the TBPs.

\subsubsection{TBP and Water Nearest Neighbors and Clusters}

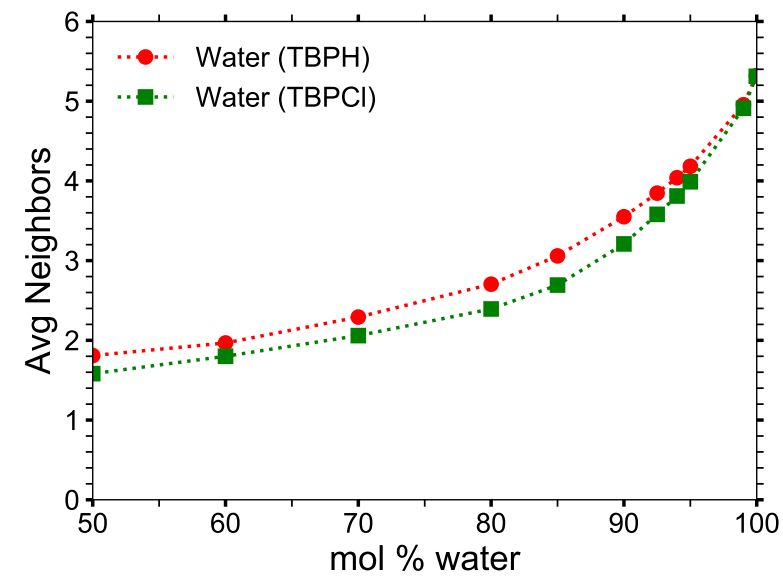

(a) Water-Water

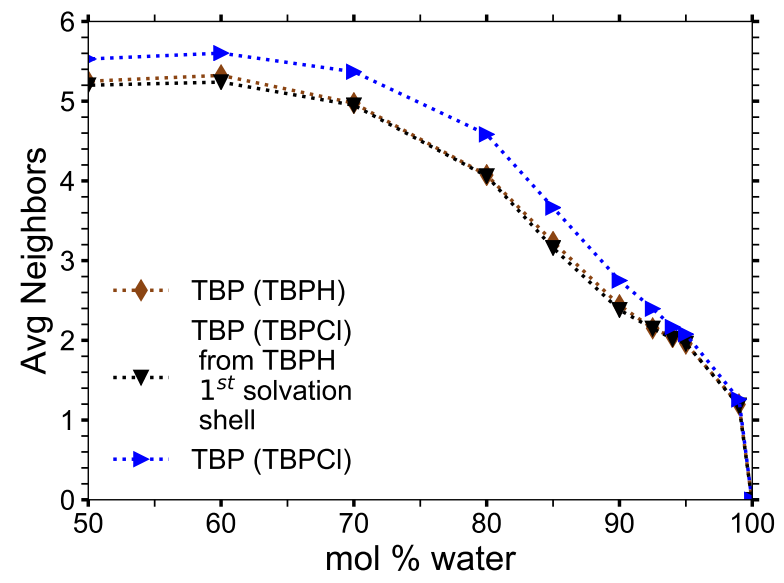

(b) $\mathrm{TBP}^{+}-\mathrm{TBP}^{+}$

Figure (3.9) Average number of nearest molecularly identical neighbors within the largest cluster: (a) water-water neighbors; (b) TBP-TBP neighbors. ${ }^{58}$

The nearest neighbors and clustering calculations were completed using data collected from the NVT production runs of the TBPCl/TBPH-water properties simulations at $300 \mathrm{~K}$. The cations are considered neighbors when the distance between $\mathrm{P}$ atoms of the two adjacent TBP molecules is within the pure TBPH or TBPCl salt distances (i.e., the radius of the first 


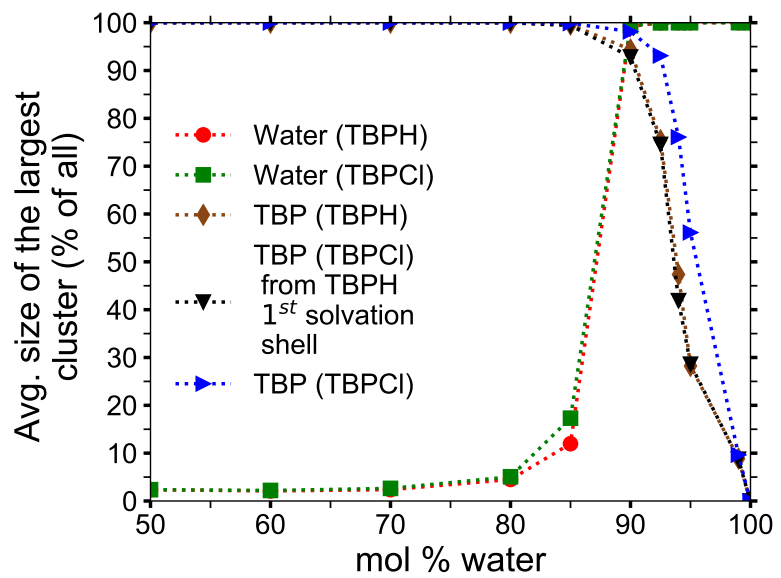

Figure (3.10) Average largest clusters of water and $\mathrm{TBP}^{+}$ions in the $\mathrm{TBPCl}$-water and TBPH-water solutions. ${ }^{58}$ The fraction of all (\%) is the fraction of the molecularly identical molecules.

$\mathrm{TBP}^{+}-\mathrm{TBP}^{+}$solvation shell), which were calculated in the simulation to be approximately $9.1 \AA$ and $9.3 \AA$, respectively. The water molecules are considered to be neighbors if they are located within $2^{(1 / 6)} \sigma_{O O}$ of each other (i.e., $2^{(1 / 6)}$ times distance that yields a zero LennardJones potential between the oxygens in the water molecules). ${ }^{62}$ For either the TBP or water molecules, if there is a network of neighbors (i.e., a path of neighbors) from one molecule to another, then this set of molecules is considered to be a single 'cluster'. These clusters are further broken down to the fraction of all of the molecularly identical molecules. For example, a water cluster with a cluster fraction of $100 \%$ means the all of the water molecules form a single cluster. The average number of neighbors of $\mathrm{TBP}^{+}$and water molecules in each cluster and the fraction of all molecules in the largest cluster are shown in Figures 3.9 and 3.10 , respectively.

The water molecules have approximately two neighbors between 50 to $70 \mathrm{~mol} \%$ water while the water pockets are forming between the TBPs interlocking arms. Once the expanding water pockets break the TBPs interlocking arms, the number of neighbors for the water increases much more rapidly as the water veins begin to form between these isolated water pockets. Water pockets form and grow slowly until around $80 \mathrm{~mol} \%$ water, then grow much more rapidly as water veins begin to develop with increasing water concentration. The water veins are the dominant water structure between 80 to 92.5 mol \% water. After $92.5 \mathrm{~mol} \%$ water, globular water clusters start to form, with the water veins still dominat- 
ing the structural formation. Once the solution reaches $94 \mathrm{~mol} \%$ water, the globular water structure is more dominant, but some water veins are still present in the solution. At $95 \mathrm{~mol} \%$ water, there is a pronounced shift in the water structure, as the globular water structure is the superior structure with a minute amount of water veins remaining. Once the water concentration surpasses $99 \mathrm{~mol} \%$ water, the solution has definitely inverted from water dissolved in $\mathrm{TBPCl} / \mathrm{TBPH}$ to $\mathrm{TBPCl} / \mathrm{TBPH}$ dissolved in water. The water vein formation and globular water structures can be visualized in the TBPCl-water solution (Figures 3.11, 3.12, and 3.13) The TBPH-water solutions images are very similar to the TBPCl-water, so the TBPH-water solutions visualizations are presented in the Appendix.

Once the TBPCl/TBPH-water solutions are diluted with water to $99 \mathrm{~mol} \%$ water, the $\mathrm{TBP}^{+}$cations on average have approximately $1.2 \mathrm{TBP}$ neighbors per cation. At high water concentrations, the TBP molecules exist as dimers of cations. At $91.1 \mathrm{~mol} \%$ water, the TBP cations prefer the trimer formation, with some large faction also existing in the quadmer formation. This TBP trimer formation is dominant from 92.5 to 95 mol \% water, where the cellulose solubility begins to decline or completely fails to dissolve cellulose in the TBPHwater and TBPCl-DMF solutions. ${ }^{8,16}$ This is especially true if the first solvation shell of TBPH is used for the TBPCl trimer calculations (i.e., the first $\mathrm{TBP}^{+}-\mathrm{TBP}^{+}$solvation shell in the $\mathrm{TBPCl}$ solution uses the $9.1 \AA$ radii of the TBPH solution instead of the actual $9.3 \AA$ radii of the $\mathrm{TBPCl}$ solution).

All the TBP cations form a single cluster at low water concentrations. As the water concentration approaches 80 to $85 \mathrm{~mol} \%$ water, the single TBP cluster begins to break apart. The TBP cluster is broken at $90 \mathrm{~mol} \%$ water, which coincides with the complete formation of the water cluster throughout the solution. The formation of a single water cluster at and above $90 \mathrm{~mol} \%$ water also occurs for alkylimidazolium-water solutions. ${ }^{62}$ The breaking of the TBP clusters is due to the prompt expansion of the water pockets that form between the TBP arms up until $80 \mathrm{~mol} \%$ water, followed by the water vein formation at $80 \mathrm{~mol} \%$ water and above. The water veins structurally transform the solution, breaking apart the interlocking TBP clusters and allowing a more probable interchange of molecules between different TBP water pockets. With increasing water concentration for the TBPCl-water solution, the structural decay of the TBP cation cluster resembles a sigmoidal decay function, with the 
largest clusters yielding approximately $9 \%$ of the total TBP atoms at $99 \mathrm{~mol} \%$ water.

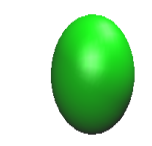

Chloride

(Cl-) anion

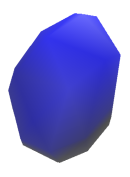

Water

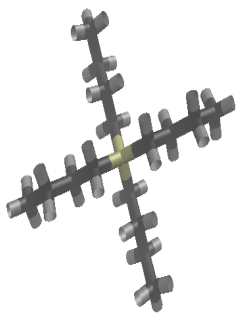

Tetrabutylphosphonium

(TBP+) cation

Figure (3.11) Depiction of molecules in the TBPCl-water solution. ${ }^{58}$ The blue-colored water is represented using an isosurface (called quicksurf in $\mathrm{VMD}^{30}$ ), which uses a volumetric Gaussian density map of the water to produce the observable surface. The TBP molecule is represented using dynamic bonds in VMD. ${ }^{30}$ TBP is colored tan, black and gray, for the phosphorus, carbon, and hydrogen atoms, respectively. The chloride is green and shown in the Van der Walls (VDW) representation. 


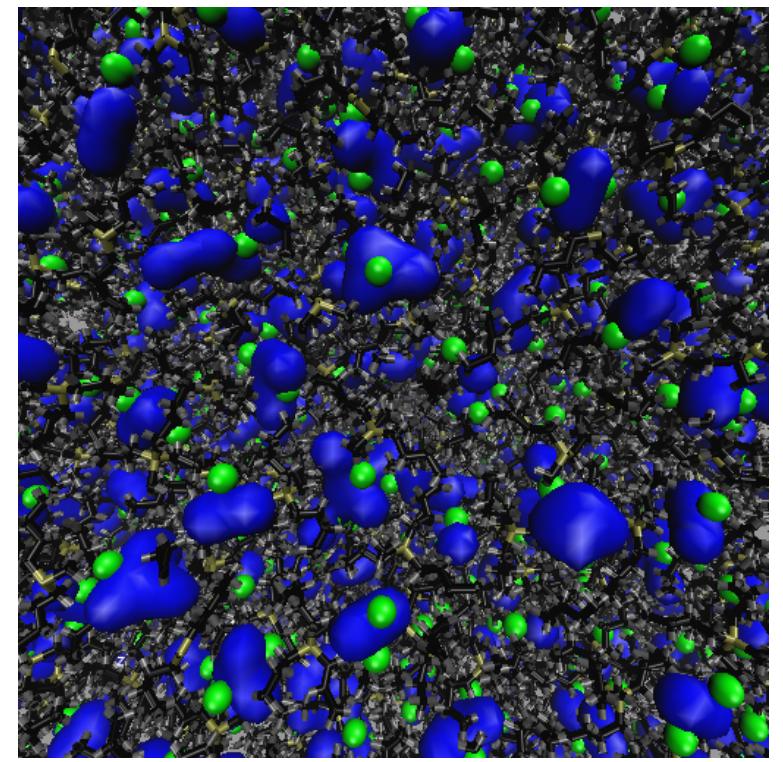

(a) $63.1 \mathrm{~mol} \%$ water

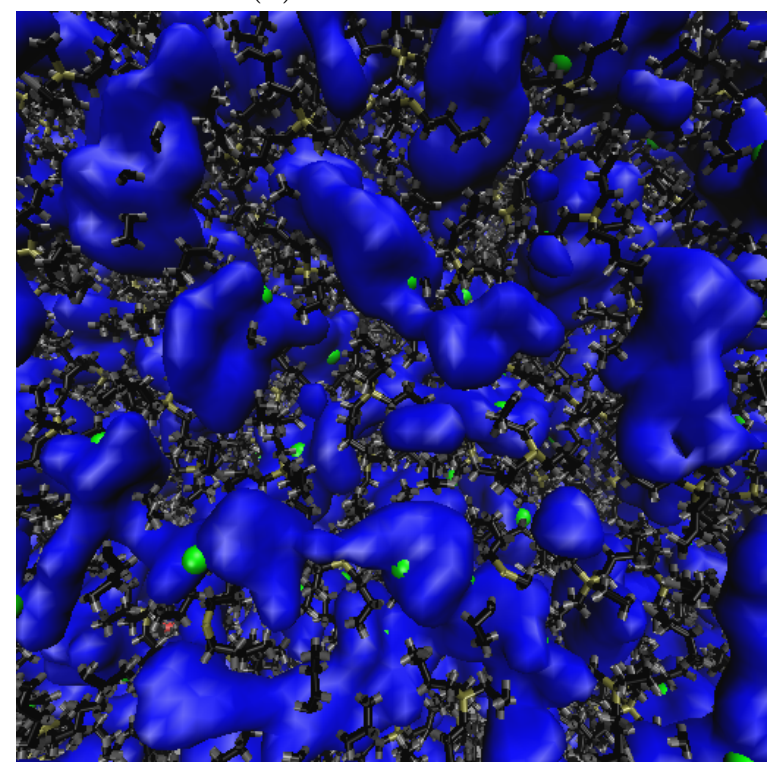

(c) $86.8 \mathrm{~mol} \%$ water

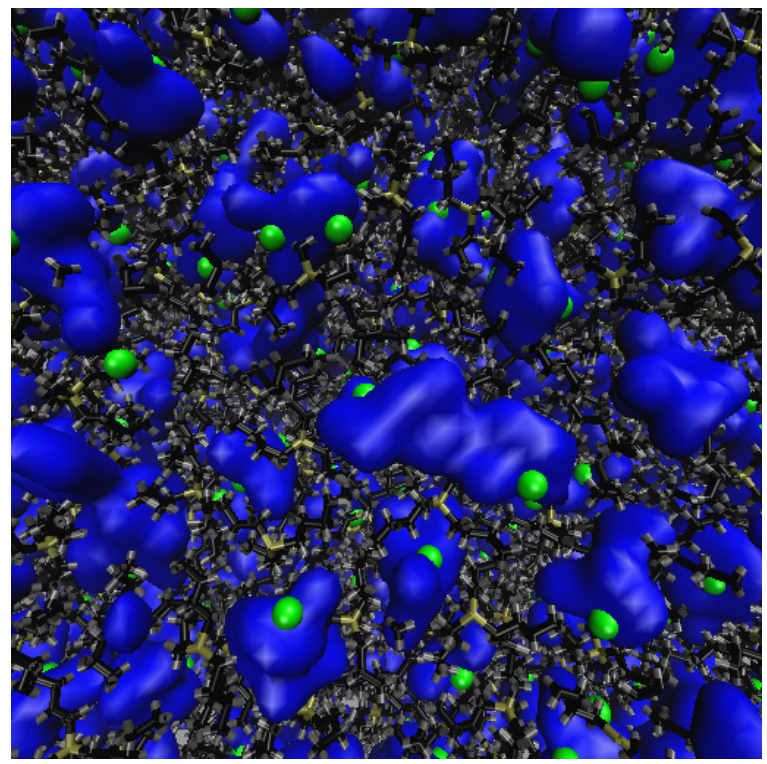

(b) $79.4 \mathrm{~mol} \%$ water

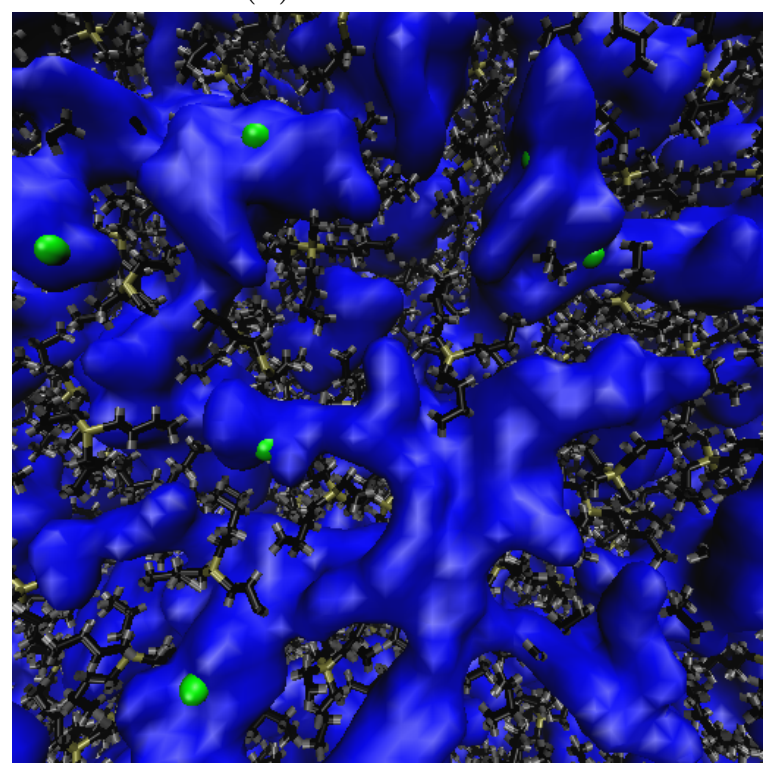

(d) $91.1 \mathrm{~mol} \%$ water

Figure (3.12) Water vein/channeling formation in TBPCl-water at $360 \mathrm{~K}$ (Part 1 of 2). ${ }^{53,54,58}$ The blue-colored water is represented using an isosurface drawing method (called quicksurf in $\mathrm{VMD}^{30}$ ), which uses a volumetric Gaussian density map of the water to produce the observable surface. The TBP molecule is represented using dynamic bonds in VMD. ${ }^{30}$ TBP is colored tan, black and gray, for the phosphorus, carbon, and hydrogen atoms, respectively. The green chloride is represented using the Van der Walls (VDW) drawing method in VMD. ${ }^{30}$ 


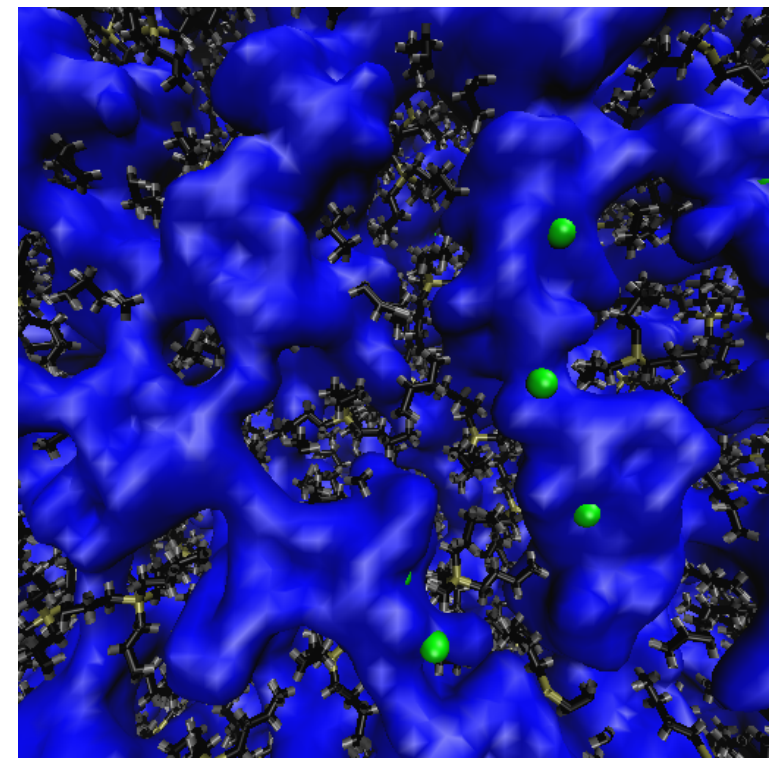

(a) $93.9 \mathrm{~mol} \%$ water

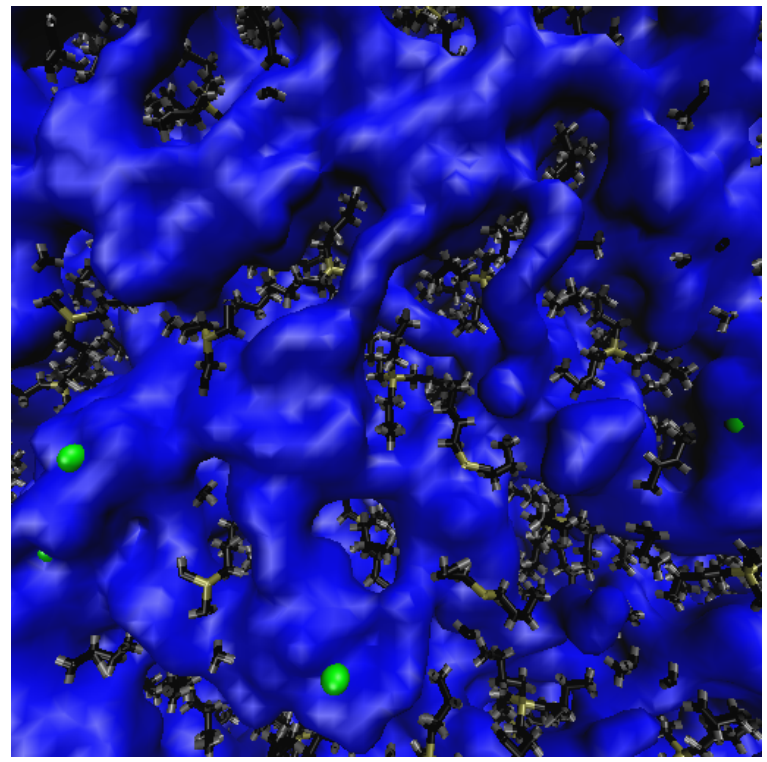

(b) $95.8 \mathrm{~mol} \%$ water

Figure (3.13) Water vein/channeling formation in TBPCl-water at $360 \mathrm{~K}$ (Part 2 of 2). ${ }^{53,54,58}$ The blue-colored water is represented using an isosurface drawing method (called quicksurf in $\mathrm{VMD}^{30}$ ), which uses a volumetric Gaussian density map of the water to produce the observable surface. The TBP molecule is represented using dynamic bonds in VMD. ${ }^{30}$ TBP is colored tan, black and gray, for the phosphorus, carbon, and hydrogen atoms, respectively. The green chloride is represented using the Van der Walls (VDW) drawing method in VMD. ${ }^{30}$ 


\subsection{Thermodynamic Data}

TBPH-water and TBPCl-water solutions with high water concentrations are liquids at room temperature. The enthalpy data for all of the concentrations were analyzed and showed smooth curves without any step changes. Thus, all the examined TBPH-water and TBPClwater solutions were liquids, confirming that the calculations for heat capacity $\left(c_{p}\right)$ and thermal expansivity $\left(\alpha_{\mathrm{p}}\right)$ are unaffected by phase changes.

\subsubsection{Excess Properties: TBPH/TBPCl-Water Solutions}

The excess volume represents the structural transformation that occurs in the system, as the water fills the void spaces between the TBP arms. The excess enthalpy of mixing shows how thermal energy is added or removed from the system from concentrating or diluting the system. The excess volumes and enthalpies were computed for these simulations at $300 \mathrm{~K}$ (see Figure 3.14), using a linear approximation method between the three temperature points of $280 \mathrm{~K}, 300 \mathrm{~K}$, and $320 \mathrm{~K}$. Both the TBPH-water and TBPCl-water solutions show a minimal deviation of approximately $4 \%$ from the ideal densities. The variance in the excess molar volume is more pronounced at around 50 to $70 \mathrm{~mol} \%$ water, as the water pockets are still being filled, and the initial random distribution of the water molecules is uneven. This uneven water distribution is held relatively constant as the water molecules are trapped between the TBPs interlocking arms within these water concentrations.

The maximum change in the excess enthalpy of mixing occurs between the interwoven TBP arms and the full solvation of the TBPs in water (60 to $99.97 \mathrm{~mol} \%$ water). In other words, the minimum excess enthalpy of mixing occurs at $60 \mathrm{~mol} \%$ water, before the water pockets are filled and begin to separate the interlocking TBP arms. The excess enthalpy of mixing can increase the solution's temperature, especially in the TBPH-water solution. Since the TBPH-water solution has a low thermal decomposition temperature, the excess

enthalpy of mixing should be considered when designing a feasible process. Substituting the TBPCl-water solution would avoid the thermal instability, as it possesses increased thermal stability. Experimental testing based on the calculated excess enthalpy is required before designing a cellulose dissolution process. 
The minimum rate of change in the excess enthalpy of mixing occurs between 60 to $80 \mathrm{~mol} \%$ water where the structural changes in the TBP clustering are slow. In this region, the difference in excess enthalpy of mixing is attributed to the anion-water hydrogen bonding and some smaller structural changes. The maximum rate of change in the excess enthalpy of mixing occurs between 80 to $99.97 \mathrm{~mol} \%$ water, due to the massive structural changes in the system, such as the breaking of the TBPs interlocking arms, water vein formation, globular water structure formation, hydrogen bonding, and other changes.

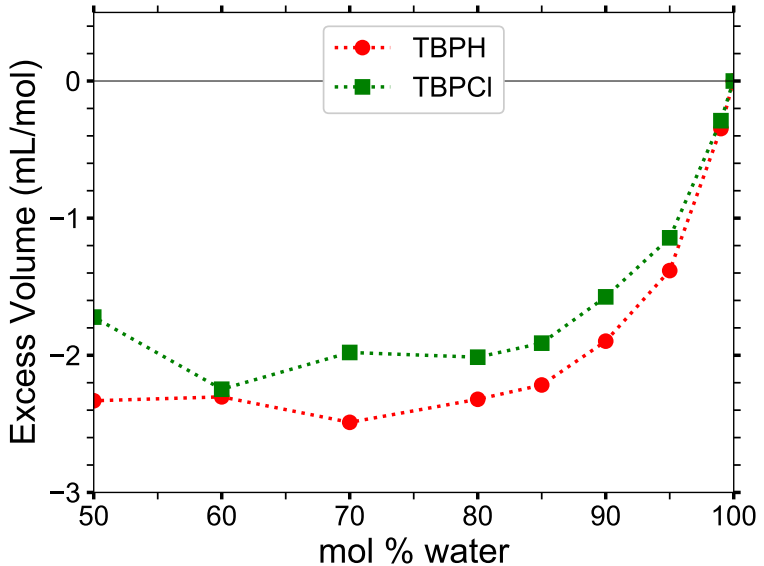

(a)

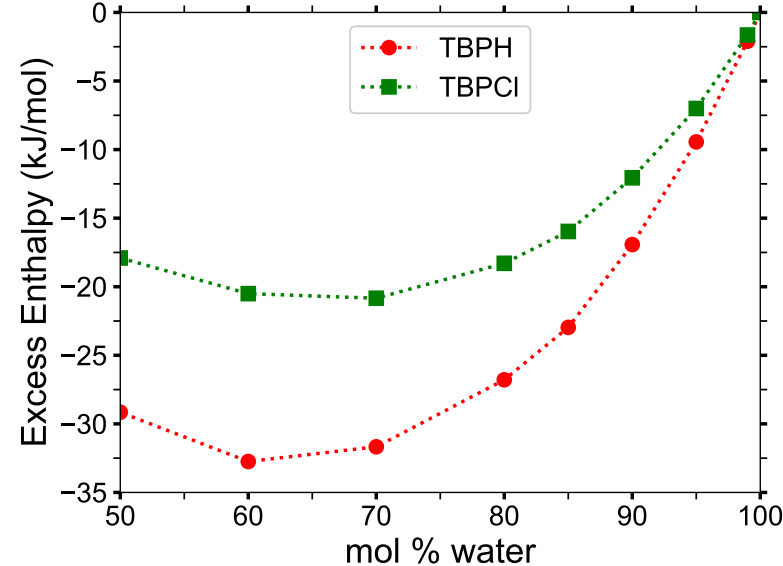

(b)

Figure (3.14) (a) Excess molar volume and (b) excess molar enthalpy of mixing in the TBPH-water and TBPCl-water solutions at $300 \mathrm{~K}$ and 1 atm. ${ }^{58}$

\subsubsection{Heat Capacity and Thermal Expansivity: TBPH/TBPCl-Water}

Both the heat capacities $\left(c_{\mathrm{p}}\right)$ and the thermal expansivities $\left(\alpha_{\mathrm{p}}\right)$ are shown in Table 3.1 as a function of water concentration. The heat capacity $\left(c_{\mathrm{p}}\right)$ is shown in both a per mass basis and per mol basis, as typically, the per gram basis is preferred in an industrial setting (see Figure 3.15). The data can be converted to a mass or mol basis by using the average molecular weight of a selected concentration. The heat capacity is linear and overlapping for both the TBPH-water and TBPCl-water solution in the per mol basis. The heat capacity on a mass basis ranges between 4.2 and $5.1 \mathrm{~kJ} \mathrm{~kg}^{-1} \mathrm{~K}^{-1}$ within an 'industrial working range' (70 to $99.97 \mathrm{~mol} \%$ water). The simulated heat capacities are higher than expected, as the known heat capacity of water is $4.184 \mathrm{~kJ} \mathrm{~kg}^{-1} \mathrm{~K}^{-1} .80$ The simulated heat capacities were scaled by 
0.82 to obtain a more accurate estimate, which is the linear scaling factor required to obtain the experimental heat capacity of water at $99.97 \mathrm{~mol} \%$ water. This scaling yields a heat capacity of 3.4 to $4.2 \mathrm{~kJ} \mathrm{~kg}^{-1} \mathrm{~K}^{-1}$, which should approximate the real heat capacities more accurately. If required, the same method of scaling can be applied to the per mol basis heat capacities to obtain more accurate numbers.

Figure 3.16 shows the thermal expansivities $\left(\alpha_{\mathrm{p}}\right)$, which are nearly constant at $0.0007 \mathrm{~K}^{-1}$ between 50 to $95 \mathrm{~mol} \%$ water. However, the thermal expansivity rapidly and dramatically changes as the solution concentration exceeds 95 mol \% water, moving to $0.0003 \mathrm{~K}^{-1}$ at $99.97 \mathrm{~mol} \%$ water. The experimental thermal expansivity for water is $0.000207 \mathrm{~K}^{-1}$ at $293 \mathrm{~K},{ }^{81}$ which can be used to scale, and more realistically approximate the simulated data in the same manner as the heat capacities.

Both the simulated heat capacities and thermal expansivities deviate from the experimental values due to imperfect models. The simulation equations are typically designed around a few properties, so the accuracy of other properties can be skewed as a result, which is the case for the present calculations. When using molecular dynamics simulations to calculate some properties, the exact accuracy of the values may vary. Still, the simulations are highly capable of identifying trends under changing conditions, and the order of magnitude of the properties. For example, they are useful for identifying trends when changing concentration, temperature, and pressure. 


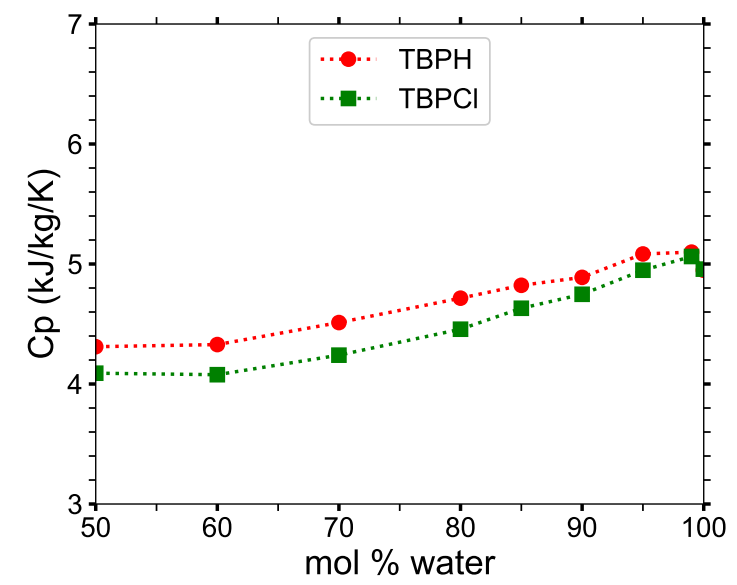

(a)

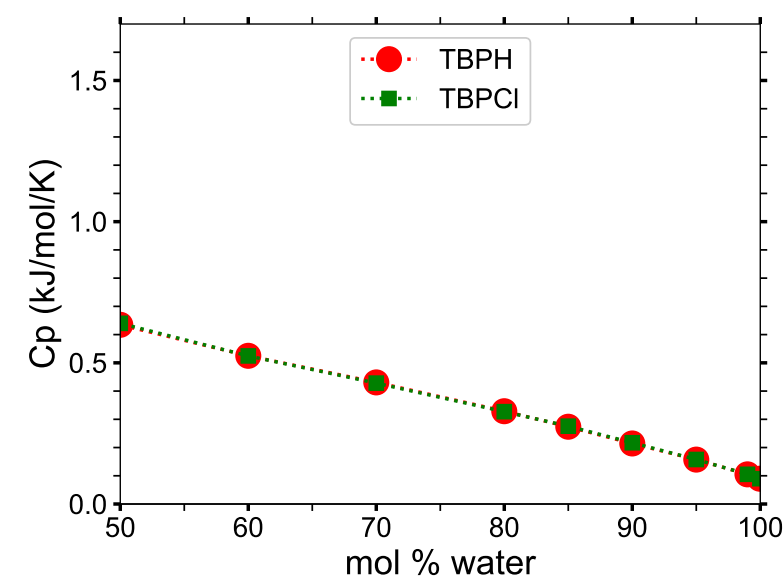

(b)

Figure (3.15) Heat capacity $\left(c_{p}\right)$ of $\mathrm{TBPH} / \mathrm{TBPCl}-$ water solutions at $300 \mathrm{~K}$ and 1 atm on a (a) per gram basis and (b) per mole basis, with overlapping TBPH-water and TBPCl-water values. ${ }^{58}$

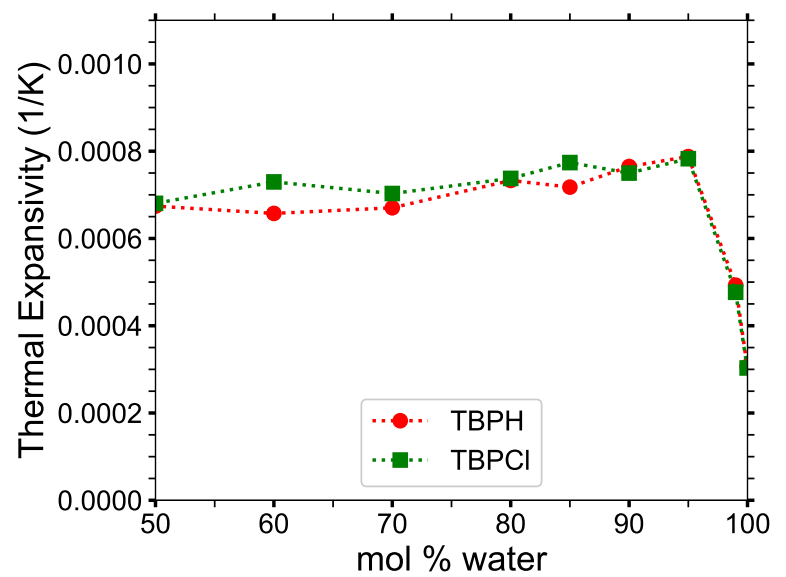

Figure (3.16) Thermal expansivity constant $\left(\alpha_{\mathrm{p}}\right)$ of the TBPH/TBPCl-water solutions at $300 \mathrm{~K}$ and 1 atm solutions. ${ }^{58}$ 


\section{Table (3.1) Heat Capacities and Thermal Expansivities of TBPH/TBPCl- Water Solutions}

\begin{tabular}{cccc}
\hline \hline & \multicolumn{3}{c}{ TBPH-water } \\
\hline $\begin{array}{c}\text { Mole \% } \\
\text { water }\end{array}$ & $\begin{array}{c}\text { Mass heat capacity } \\
c_{p}, \mathrm{~J} \mathrm{~g}^{-1} \mathrm{~K}^{-1}\end{array}$ & $\begin{array}{c}\text { Molar heat capacity } \\
c_{p}, \mathrm{~J} \mathrm{~mol}^{-1} \mathrm{~K}^{-1}\end{array}$ & $\begin{array}{c}\text { Thermal expansivity } \\
\alpha_{p}, 10^{-4} \mathrm{~K}^{-1}\end{array}$ \\
\hline 50.0 & $4.31 \pm 0.17$ & $634.7 \pm 25.2$ & $6.74 \pm 0.39$ \\
60.0 & $4.33 \pm 0.25$ & $524.9 \pm 29.8$ & $6.57 \pm 0.28$ \\
70.0 & $4.51 \pm 0.21$ & $430.7 \pm 20.1$ & $6.70 \pm 0.26$ \\
80.0 & $4.72 \pm 0.17$ & $328.7 \pm 12.2$ & $7.33 \pm 0.36$ \\
85.0 & $4.82 \pm 0.16$ & $273.7 \pm 9.2$ & $7.18 \pm 0.21$ \\
90.0 & $4.89 \pm 0.18$ & $214.4 \pm 8.1$ & $7.65 \pm 0.30$ \\
95.0 & $5.08 \pm 0.16$ & $157.3 \pm 5.1$ & $7.88 \pm 0.23$ \\
99.0 & $5.10 \pm 0.17$ & $105.0 \pm 3.4$ & $4.93 \pm 0.24$ \\
$99.97^{a}$ & $4.95 \pm 0.17$ & $89.6 \pm 3.0$ & $3.07 \pm 0.21$ \\
\hline
\end{tabular}

\begin{tabular}{cccc}
\hline \hline & \multicolumn{3}{c}{ TBPCl-water } \\
\hline Mole $\%$ & $\begin{array}{c}\text { Mass heat capacity } \\
\text { water }\end{array}$ & $\begin{array}{c}\text { Molar heat capacity } \\
c_{p}, \mathrm{~J} \mathrm{~g} \mathrm{~mol}^{-1} \mathrm{~K}^{-1}\end{array}$ & $\begin{array}{c}\text { Thermal expansivity } \\
\alpha_{p}, 10^{-4} \mathrm{~K}^{-1}\end{array}$ \\
\hline 50.0 & $4.09 \pm 0.21$ & $639.9 \pm 32.6$ & $6.80 \pm 0.22$ \\
60.0 & $4.08 \pm 0.19$ & $524.5 \pm 24.5$ & $7.30 \pm 0.22$ \\
70.0 & $4.24 \pm 0.19$ & $428.2 \pm 19.5$ & $7.03 \pm 0.22$ \\
80.0 & $4.46 \pm 0.20$ & $327.1 \pm 14.5$ & $7.38 \pm 0.24$ \\
85.0 & $4.63 \pm 0.21$ & $275.7 \pm 12.7$ & $7.74 \pm 0.36$ \\
90.0 & $4.75 \pm 0.12$ & $217.0 \pm 5.6$ & $7.50 \pm 0.22$ \\
95.0 & $4.95 \pm 0.20$ & $157.7 \pm 6.2$ & $7.83 \pm 0.28$ \\
99.0 & $5.06 \pm 0.13$ & $105.2 \pm 2.7$ & $4.77 \pm 0.31$ \\
$99.97^{a}$ & $4.96 \pm 0.21$ & $89.8 \pm 3.8$ & $3.03 \pm 0.42$ \\
\hline \hline
\end{tabular}

${ }^{a}$ Infinite-dilution simulation

Data for $p=1 \mathrm{~atm}$ and $T=300 \mathrm{~K}^{58}$

The uncertainty is calculated from the maximum slope change between the simulations at different temperatures, due to the standard deviation from the averaged parameter used in the calculations. The data represents a single simulation at each temperature. 


\subsection{Diffusion Properties: TBPH/TBPCl-Water Solutions}

The anomalous diffusion coefficients of all molecules in the TBPCl-water solution are shown in Figure 3.17 and Table 3.2. The reported anomalous diffusion coefficients are based on the particle-averaged TAMSDs, $\left\langle\overline{\delta^{2}}(\Delta)>\right.$, fitted to Equation 2.8. The $\alpha$ coefficient shows a large change from 80 to $92.5 \mathrm{~mol} \%$ water, demonstrating a dramatic transition from a subdiffusive system to a near normal diffusive system (i.e., from $\alpha \approx 0.34$ to $\approx 0.93$ ). The $K_{\alpha}$ coefficient also increases rapidly in this region and continues to increase as the system approaches pure water. In the region from 80 to $92.5 \mathrm{~mol} \%$ water, the diffusion increases at least an order of magnitude. Both these coefficients indicate the average diffusion continues to increase as the system moves toward a pure co-solvent system. However, once the diffusion regime very closely approaches normal diffusion (i.e., approximately $\alpha \geq 0.96$ ), both the TBPCl-water and TBPH-water solution is no longer able to dissolve cellulose. ${ }^{8,16}$ In this study, the cation, anion, and water diffusivities all trend together, in agreement with the cation and anion diffusivities from Thompson et al. ${ }^{82}$ The TAMSD versus time for each individual molecule displayed highly non-linear trends, resulting in anomalous diffusion at many of the water concentrations, which is not uncommon in ILs because of the added Coulombic forces (see Table 3.2 and Figures 3.19, and 3.21). ${ }^{62,83}$

The rapid change in the diffusion regime is attributed to the breaking of the $\mathrm{TBP}^{+}$'s interlocking arms, water vein formation, and the weakening Coulombic forces. Once the $\mathrm{TBP}^{+}$'s interlocking arms are broken, the water, hydroxide, and chloride can "tunnel" through the

water veins, further increasing their mobility. ${ }^{84,85}$ The transition from the subdiffusive to the normal diffusive regime can be visualized in the ergodicity breaking parameter, $\chi$, plots (see Figure 3.20). At shorter time scales, less than a nanosecond, sufficient time-averaging in TAMSDs is available. In this timeframe, $\chi$ is at least an order of magnitude lower in the 80 to $99.97 \mathrm{~mol} \%$ water region, also indicating that the subdiffusive region is becoming or is a normal diffusive region. The anion and water have larger $\chi$ values, which plateau in the subdiffusive region, inferring they may be more affected by the trapping and caging in the subdiffusive region than the TBP molecule. At all concentrations and at longer times scales, all molecules show increasing $\chi$ values, which is in part due to a less statistical averaging 
from the TAMSDs in this region. This higher variance at larger time scales begins to taper off at $80 \mathrm{~mol} \%$ water and stabilize at $92.5 \mathrm{~mol} \%$ water, suggesting that some of the high variances could stem from faster diffusion in or along the water veins before water forms a more globular structure.

The Grotthuss mechanism or proton jumping via a hydrogen-bonding network can have a significant impact on the diffusion of the TBPH-water system. In this case, the hydrogenbonding network is the water and hydroxide molecules. ${ }^{86}$ The effect of the Grotthuss mechanism on the $\mathrm{OH}^{-}$and water diffusion was considered when selecting a non-reactive force field. The reactive force field (ReaxFF) data from Zhang et al. ${ }^{86}$ are shown on the generalized diffusion coefficient plot (see Figure 3.18), and are considered to be a likely part of the overall diffusion process, especially in the water concentration range where cellulose is soluble. While the Grotthuss mechanism likely increases the diffusivity of the TBPH-water solution, it can only do it effectively across the entire system once the $\mathrm{TBP}^{+}$'s interlocking arms are broken and the water pockets become connected. Therefore, the non-reactive force field that was used in this study is sufficient to demonstrate the diffusive regime changes in these solutions.

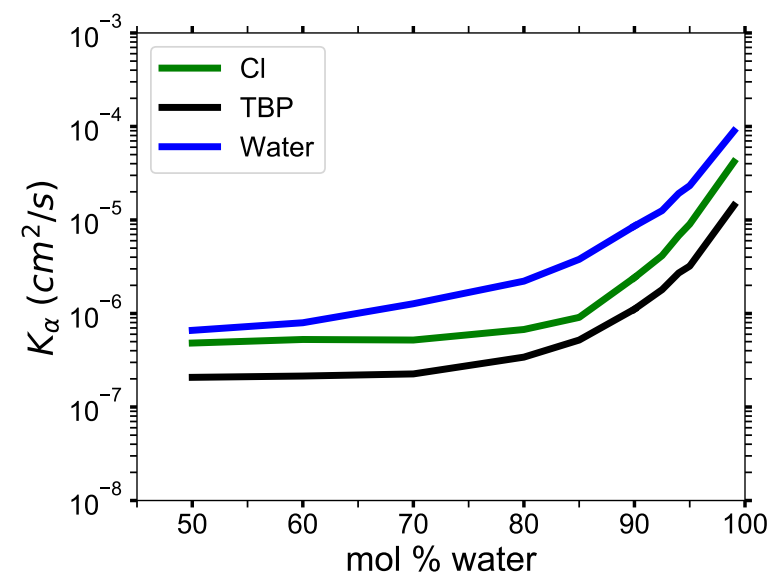

(a)

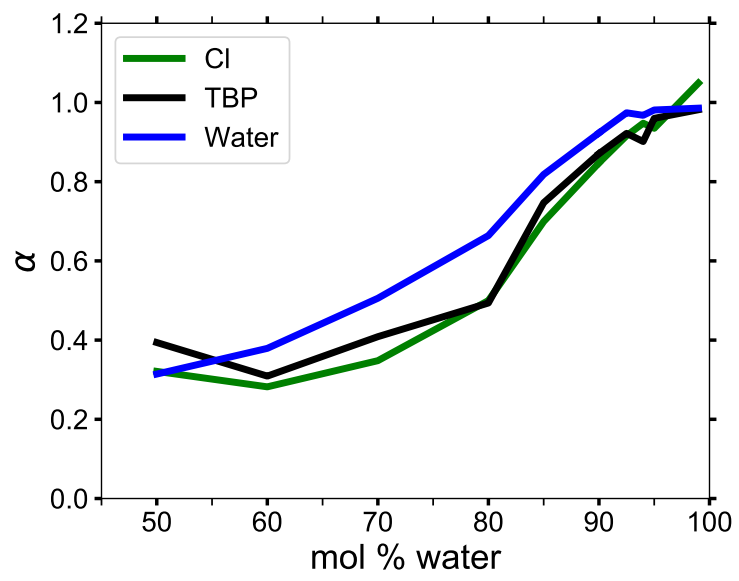

(b)

Figure (3.17) Anomalous diffusion coefficients in TBPCl-water at $p=1$ atm and $T=300 \mathrm{~K}$ as a function of water concentration: ${ }^{58}$ (a) generalized diffusion coefficient, $K_{\alpha}$; (b) anomalous diffusion exponent, $\alpha$. 


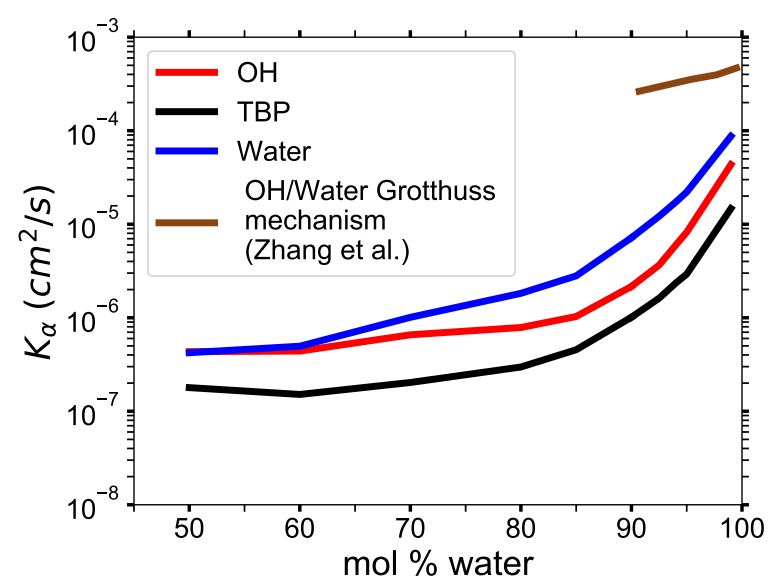

(a)

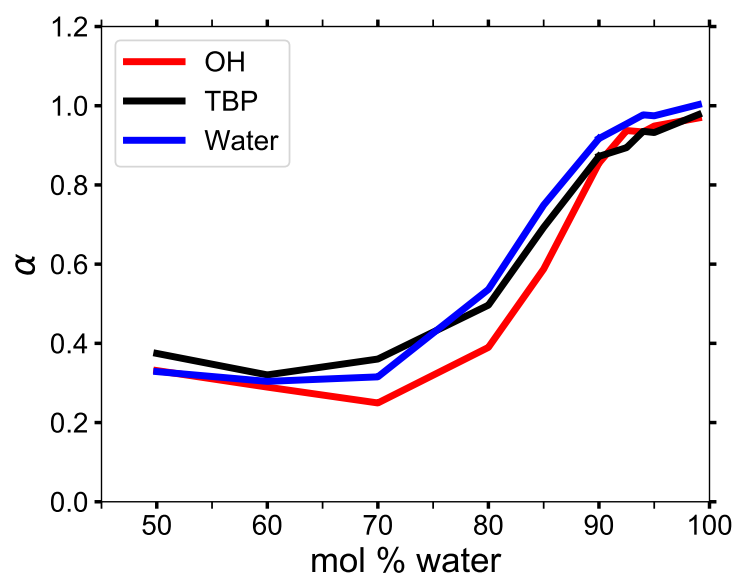

(b)

Figure (3.18) Anomalous diffusion coefficients in TBPH-water at $p=1$ atm and $T=300 \mathrm{~K}$ as a function of water concentration: ${ }^{58}$ (a) generalized diffusion coefficient, $K_{\alpha}$; (b) anomalous diffusion exponent, $\alpha$. The Grotthuss mechanism ReaxFF data was fitted to the anomalous diffusion equation with an assumed $\alpha$ value of 1 (original data from Zhang et al. ${ }^{86}$ ).

Table (3.2) TBPCl anomalous diffusion coefficients of water, $\mathrm{TBP}^{+}$, and $\mathrm{Cl}^{-}$

\begin{tabular}{ccccccc}
\hline \hline & \multicolumn{6}{c}{$K_{\alpha}\left(10^{-6} \mathrm{~cm}^{2} \mathrm{~s}^{-1}\right) / \alpha\left(1 / s^{\alpha}\right)$} \\
\cline { 2 - 7 } Mole $\%$ & $K_{\alpha} \mathrm{O}$ & $\alpha$ & $K_{\alpha}$ & $\alpha$ & $K_{\alpha}$ & $\alpha$ \\
\hline 50.0 & 0.660 & 0.314 & 0.208 & 0.394 & 0.483 & 0.321 \\
60.0 & 0.796 & 0.379 & 0.214 & 0.309 & 0.527 & 0.282 \\
70.0 & 1.27 & 0.506 & 0.226 & 0.409 & 0.520 & 0.348 \\
80.0 & 2.2 & 0.664 & 0.341 & 0.494 & 0.674 & 0.500 \\
85.0 & 3.79 & 0.818 & 0.520 & 0.747 & 0.909 & 0.699 \\
90.0 & 8.61 & 0.923 & 1.11 & 0.871 & 2.42 & 0.847 \\
92.5 & 12.6 & 0.974 & 1.80 & 0.922 & 4.16 & 0.916 \\
94.0 & 19.2 & 0.968 & 2.71 & 0.901 & 6.79 & 0.947 \\
95.0 & 23.3 & 0.981 & 3.21 & 0.960 & 9.02 & 0.935 \\
99.0 & 89.3 & 0.986 & 14.3 & 0.982 & 41.8 & 1.05 \\
$99.97^{a}$ & 141 & 0.991 & -- & -- & -- & -- \\
\hline \hline
\end{tabular}

${ }^{a}$ Infinite-dilution simulation for TBPCl

Data for $p=1 \mathrm{~atm}$ and $T=300 \mathrm{~K}^{58}$ 


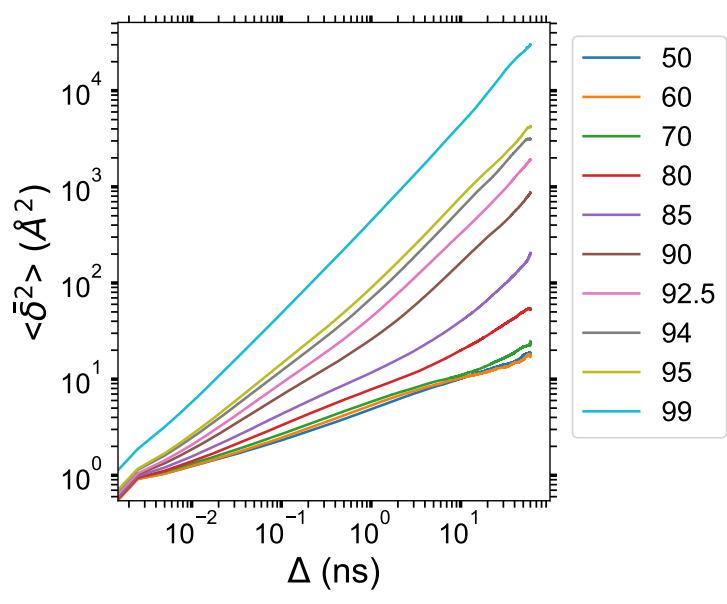

(a) $\mathrm{Cl}^{-}$

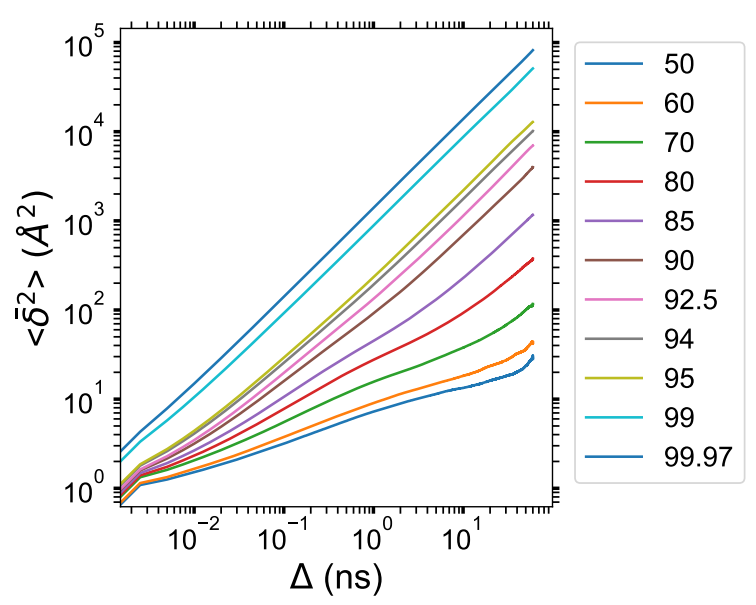

(b) Water

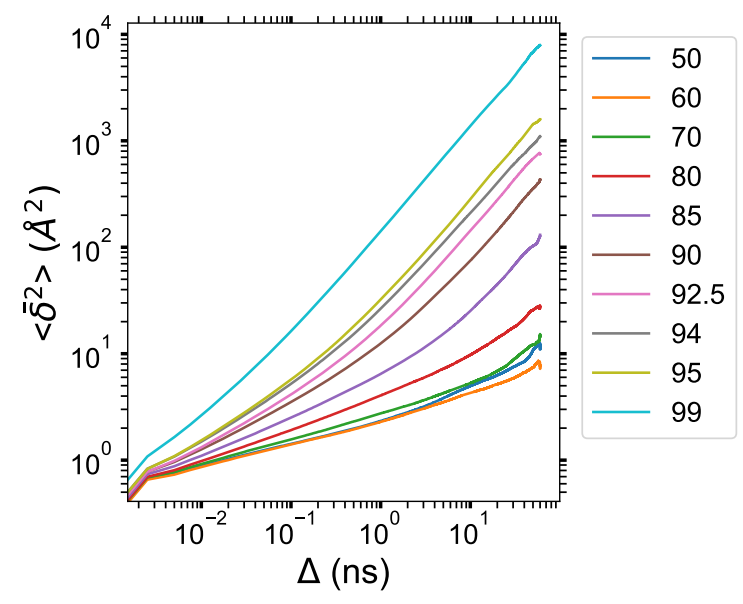

(c) $\mathrm{TBP} \mathrm{P}^{+}$

Figure (3.19) Particle-averaged TAMSDs of the TBPCl-water solution at $p=1 \mathrm{~atm}$ and $T=300 \mathrm{~K}$ as a function of water concentration. ${ }^{58}$ 


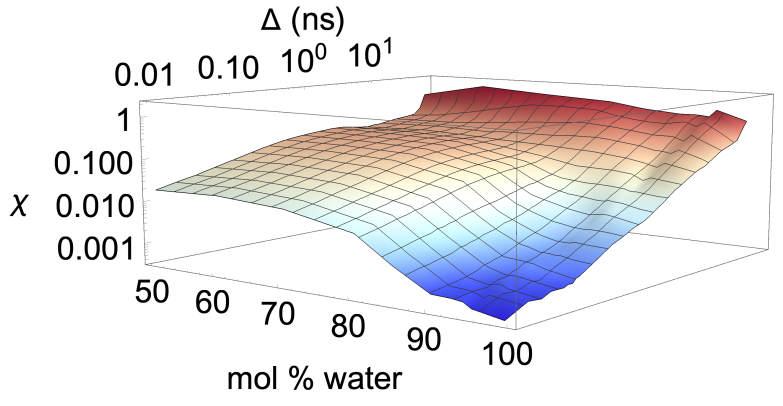

(a) $\mathrm{Cl}^{-}$

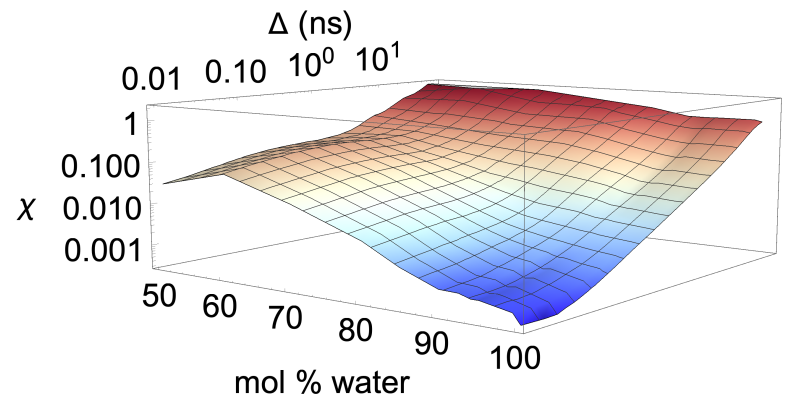

(b) Water

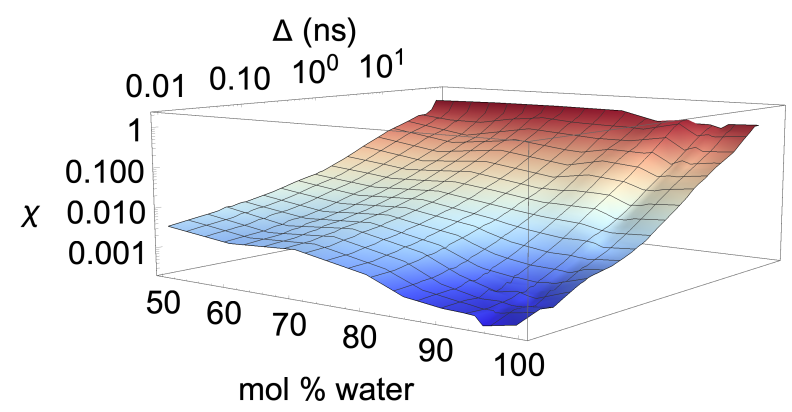

(c) $T B P^{+}$

Figure (3.20) Ergodicity breaking parameter $(\chi)$ in TBPCl-water at $p=1$ atm and $T=$ $300 \mathrm{~K}$ as a function of water concentration and lag time. ${ }^{23,58}$ 
The particle-averaged TAMSDs for the TBPCl-water system are provided in Figure 3.19. The diffusive regime can be further defined by evaluating the MSD vs. particle-averaged TAMSD in Figure 3.21. Due to the high variance at low water concentrations from the trapping and caging by the TBP molecules, together with the MSDs and particle-averaged TAMSDs overlapping, the subdiffusion appears to stem from random and changing fractal geometries of a percolation cluster. ${ }^{55,56,87}$ Once the solution approaches 80 to 85 mol \% water, fractal geometries of a percolation cluster, or trapping and caging, becoming diminished by water vein formation, leading to the more linear particle-averaged TAMSDs found in normal diffusion. The cellulose dissolution appears to be bounded in the water vein formation region, which is essentially the percolation transition region. This bounded area is noticeable in the clustering data, the simulation 'snapshots', and the anomalous diffusion exponent (see Figures 3.10, 3.12, 3.13, and 3.17). At $80 \mathrm{~mol} \%$ water, the water clustering increases due to water vein formation throughout the system, which allows the anomalous diffusion exponent to increase. The water vein formation eliminates the trapping and caging structure of the TBP arms, increasing the system's overall diffusion, and shifting the anomalous diffusion exponent to a higher value approaching a normal diffusive regime (i.e., $\alpha=1$ ). The TBPHwater solution shows similar results, and the data are available in the Appendix (see Table 7.10 and Figures 7.14, 7.15, 7.16, 7.17, 7.18, and 7.19). 


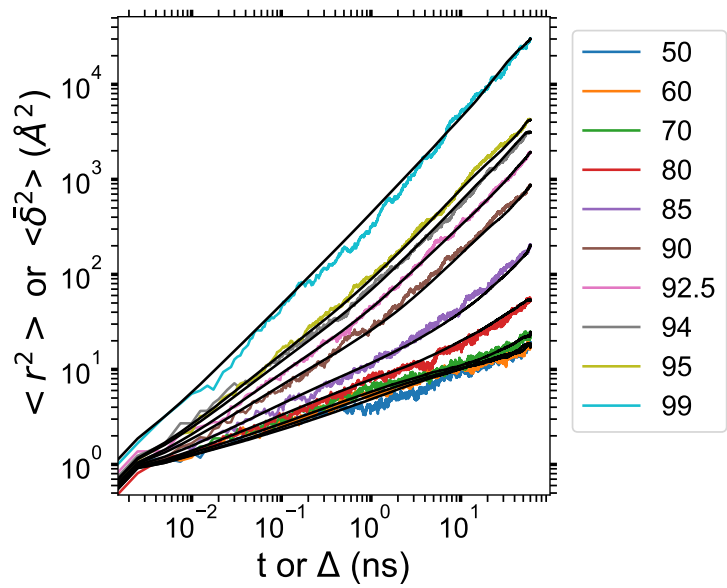

(a) $\log$ scale $\mathrm{Cl}^{-}$

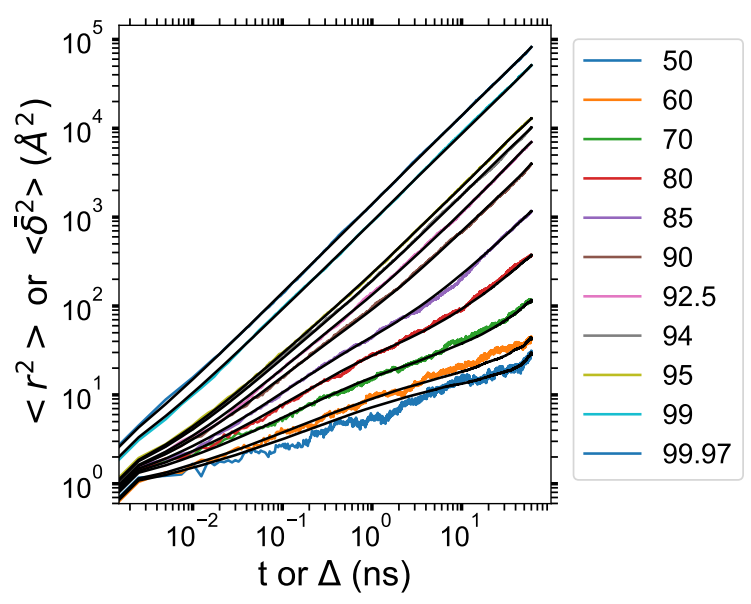

(c) Log scale Water

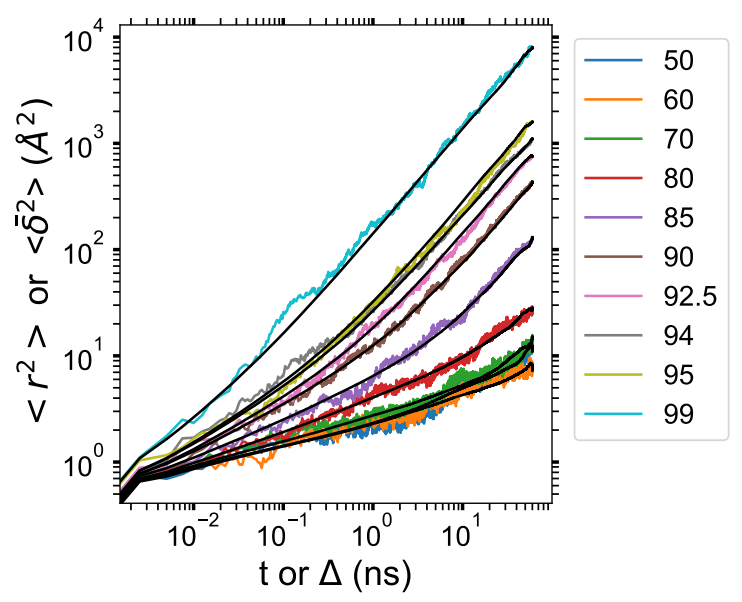

(e) Log scale $T B P^{+}$

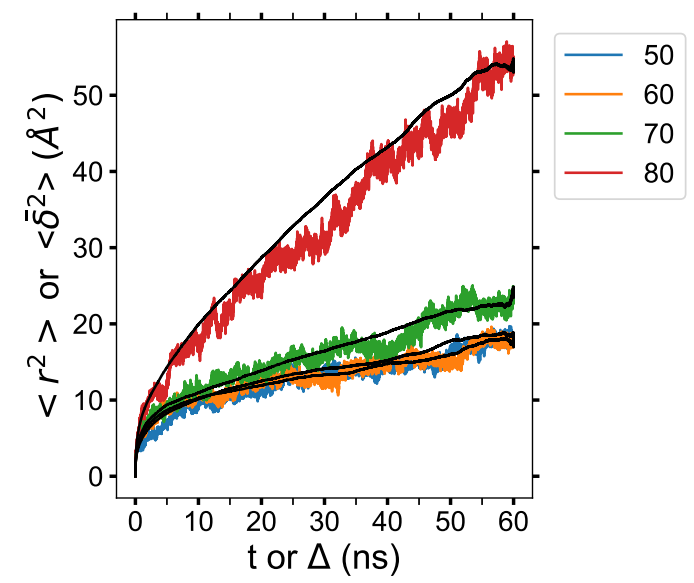

(b) Linear scale $\mathrm{Cl}^{-}$

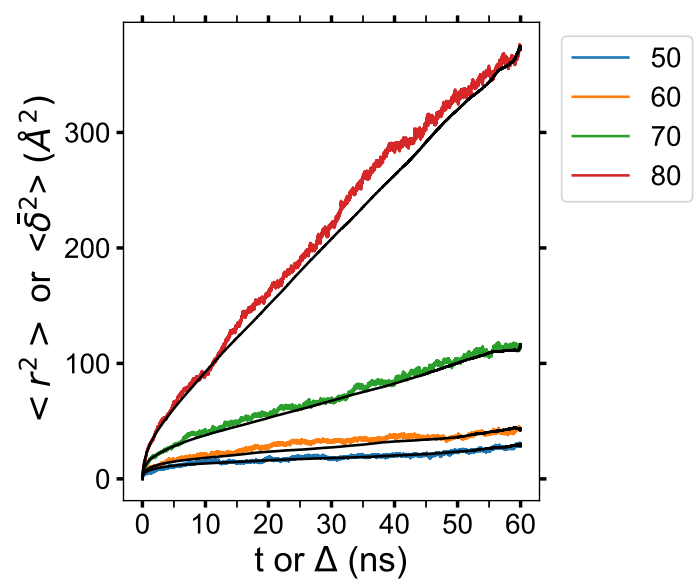

(d) Linear scale Water

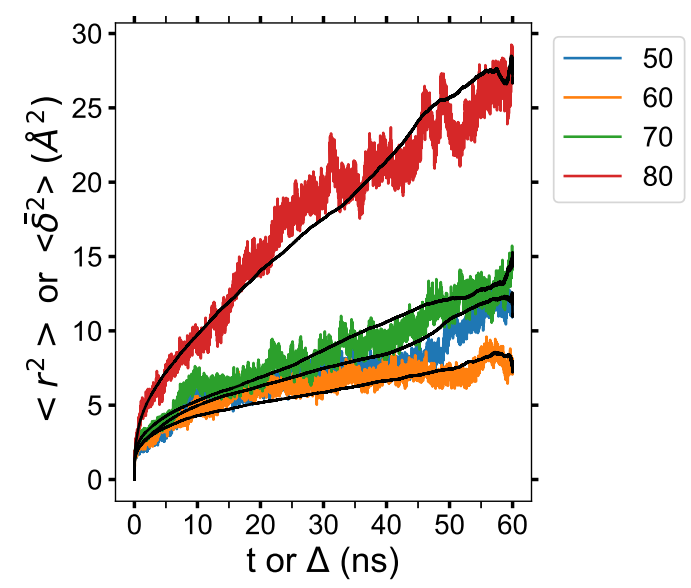

(f) Linear scale $T B P^{+}$

Figure (3.21) MSD vs. particle-averaged TAMSD of the water, TBP and Cl in TBPClwater at $p=1 \mathrm{~atm}$ and $T=300 \mathrm{~K}$ as a function of water concentration. ${ }^{58}$ The colored lines are the MSDs, and the black lines are the particle-averaged TAMSDs. 


\subsection{Overview: Long Simulations with the Small $I \beta$ Cellulose Bun- dle in TBPCl-water}

Despite the slow cellulose dissolution due to the moderate temperature, critical data could be obtained about the cellulose dissolution process. The limited cellulose breakdown is primarily attributed to the lower temperature simulations and the computational limits of the system size. The radius of gyration was calculated and was essentially constant, which is accredited to only a few cellulose strands partially dissolving, and the larger size of the bundle.

The key findings of this study are summarized below and explained in more detail in their respective sections. An estimated cellulose dissolution profile was generated for the $\mathrm{TBPCl}-$ water solution, in which the cellulose solubility appears to decrease with added water. This study determined that critical hydrogen bonding lifetime values must be maintained between the chloride and the cellulose's hydroxyl groups that are responsible for its intra-strand hydrogen bonding, in order for cellulose dissolution to occur. The hydrogen bonding lifetime threshold between the chloride-cellulose hydroxyl groups appear to be directly related to cellulose dissolution. The simulations calculated the TBP-cellulose strand pairwise energy, which is favorable (negative) and potentially net negative during the entire cellulose dissolution process. The TBP-cellulose strand pairwise energy shows a favorable thermodynamic pathway for cellulose dissolution in the TBPCl-water solution. Lastly, a cooperative cellulose dissolution mechanism is visualized and determined from these simulations, in which water appears to assist in the cellulose dissolution.

\subsection{The Extent of Cellulose Dissolution in TBPCl-water}

Each cellulose strand contains 12 glycans (12 glucose units), and they are numbered in a linear order from 1 to 12 , in the same direction for every strand. The centers of mass (COMs) of every glycan unit were calculated. For each strand, the distance between the COMs was measured for each matching glycan number, saving the minimum value or nearest neighbor distance between each of the 18 strands (i.e., 17 values per glycan number per strand). For a given glycan number, the maximum nearest neighbor distance is simply the largest of the 
nearest neighbor distance for all the strands (i.e., the largest nearest neighbor value for the 18 strands). In other words, the maximum nearest neighbor distance is the maximum distance between each nearest COMs with a matching glycan number. The average nearest neighbor distance is the nearest neighbor length for each similarly numbered glycan averaged over the different cellulose strands. Only the maximum COM separation distances of the glycans nearest neighbor are displayed instead of the average COM distances, as averaging the data at this stage of the dilution process statistically minimizes any useful results.

\subsubsection{Cellulose Dissolution in TBPCl-water}

In this work, the amount of dissolved cellulose was calculated using the pure water simulation at $10,200,400,600 \mathrm{~ns}$ as a mathematical basis to determine if each glycan is dissolved in the solution. At these time points, the overall average of the maximum nearest neighbor distances was $6.51 \AA$, the maxima of all the values were $6.96 \AA$, with a standard deviation of $0.16 \AA$. The total glycan COM separation distance determining cellulose dissolution was $7.16 \AA$, four standard deviations from the average, providing a statistically significant separation.

The simulation data appear to be more representative of the TBPCl-DMF experimental data, ${ }^{8}$ and not statistically maximizing at any specific concentration (see Figures 1.1b, 3.22, and 7.20). The TBPCl-water simulation shows a rapidly decreasing cellulose solubility after $79.4 \mathrm{~mol} \%$ water. Figure $3.22 \mathrm{~b}$ shows a very linear cellulose dissolution rate at the simulated time scales. The cellulose dissolution rate could be many orders of magnitude higher than experimental data suggest, and it could tail off rapidly at larger time scales due to the solvent becoming saturated (see Figure 3.22b and Table 7.11). ${ }^{8,16}$ However, this dissolution rate should be reduced for the larger cellulose bundles (i.e., more inter-strand hydrogen bonds and a lower fraction of glycans at the ends of the bundle), and rapidly decay as the cellulose strands separate from the bundle and interact with other dissolved strands or cellulose bundles, which was not seen in these simulations. The dissolution rates appear clustered, according to their ability to dissolve cellulose, grouped with the low water concentrations, (63.1 to $79.4 \mathrm{~mol} \%$ water), the middle water concentrations (86.8 to $91.1 \mathrm{~mol} \%$ water), and the high water concentrations (93.9 to $100 \mathrm{~mol} \%$ water). An anomalous to near-normal diffusion regime transition appears to profoundly impact the middle group where the water 
veins form throughout the solution, from roughly 80 to $92.5 \mathrm{~mol} \%$ water. ${ }^{58}$ The diffusion regime change with the formation of water veins increases the diffusion of the solvent by approximately an order of magnitude, which could partially explain the cellulose solubility extension into the water concentrations above $79.4 \mathrm{~mol} \%$ water, ${ }^{58}$ even with water decreasing the cellulose solubility. The experimental cellulose dissolution profile is estimated to look like Figure 3.22a based on these simulations and the experimental data from Figure 1.1b. ${ }^{8}$ However, the actual experimental cellulose dissolution profile could be different, as these simulations are far from reaching equilibrium.

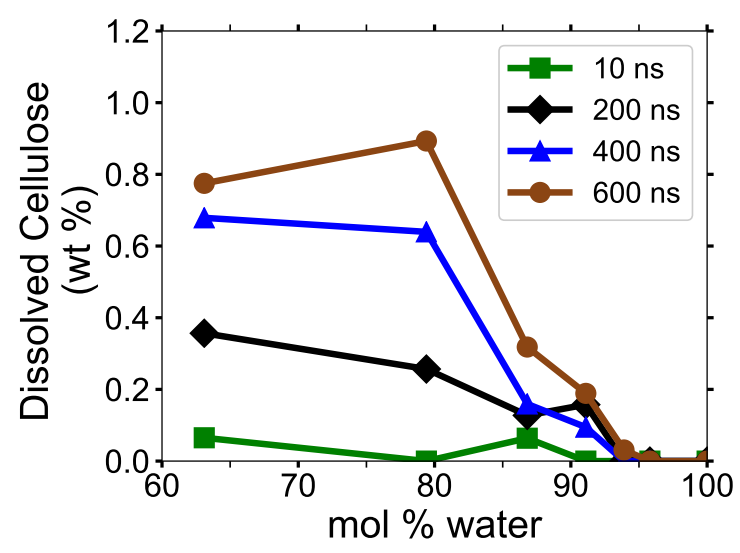

(a) Cellulose dissolution vs. water concentration

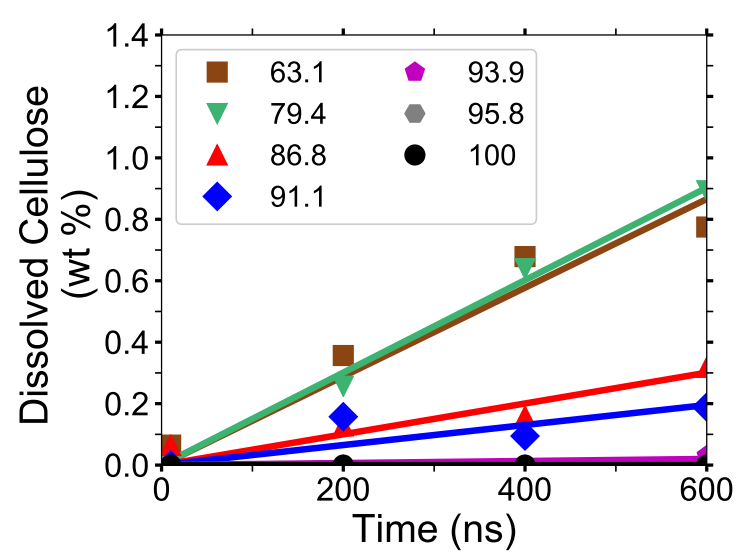

(b) Cellulose dissolution vs. time (dissolution rates)

Figure (3.22) The simulated cellulose dissolution concentrations for the TBPCl-water solution at $360 \mathrm{~K}$ : (a) cellulose dissolution vs. water concentration; (b) Cellulose dissolution vs. time (dissolution rates). The legend in plot $\mathrm{b}$ indicates the water concentration in mol $\%$ water for the $\mathrm{TBPCl}$-water solutions, while the slopes convey the cellulose dissolution rate. ${ }^{22}$

\subsubsection{Cellulose Strand Separation in TBPCl-water}

The maximum nearest neighbor distances between matching glycan numbers are an indicator of a cellulose strand separation from the cellulose bundle and hence cellulose dissolution (see Figure 3.23). The most probable strands to separate are the yellow and pink strands (i.e., first layer strands), with the yellow strands separating first in the majority of the simulations (see Figures 7.21, 7.22, 7.23, and 7.24). The most probable strands to separate also have the lowest intra-strand and inter-strand hydrogen bonding and the highest hydrogen bonding 
with the solvent, which are the first layer strands and the corner strands (see Figures 3.2, $3.3,3.4$, and 3.5$)$.

During the dissolution process, strands can separate and partially or entirely reform. The more significant the cellulose strand separation, the higher the chance the strand will only partially reform, increasing the probability of further separation. This partial strand reformation is seen at $91.1 \mathrm{~mol} \%$ water between 200 to $400 \mathrm{~ns}$, at both ends of the glycan numbers. In general, the cellulose strands proceed to dissolve, despite some strand reformation. The ends are more likely to peel off than the center of the cellulose bundles, which is an expected result since the ends have fewer hydrogen bonds stabilizing them. The 63.1 and $79.4 \mathrm{~mol} \%$ water simulations do not always show the furthest strand separation, despite having the highest concentration of dissolution, which means that they have more separating strands than the $91.1 \mathrm{~mol} \%$ water solution. 


\begin{tabular}{|c|c|c|c|c|}
\hline -レ- $63.1 \mathrm{~mol} \%$ & $-t-86.8 \mathrm{~mol} \%$ & - - $93.9 \mathrm{~mol} \%$ & $-\infty-$ & $100 \mathrm{~mol} \%$ \\
\hline -- - $79.4 \mathrm{~mol} \%$ & $-x-91.1 \mathrm{~mol} \%$ & $-\bullet 95.8 \mathrm{~mol} \%$ & -4 & $\begin{array}{l}100 \mathrm{~mol} \% \\
\text { at } 10 \mathrm{~ns}\end{array}$ \\
\hline
\end{tabular}

(a) Legend

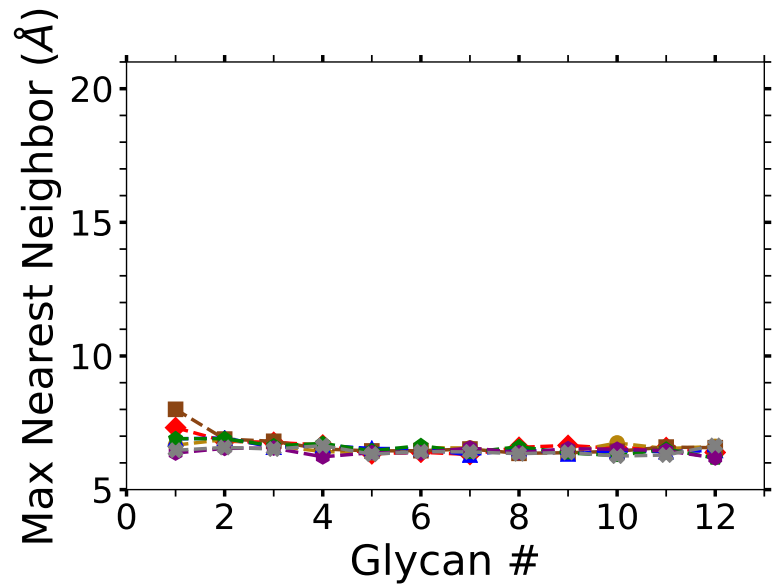

(b) $10 \mathrm{~ns}$

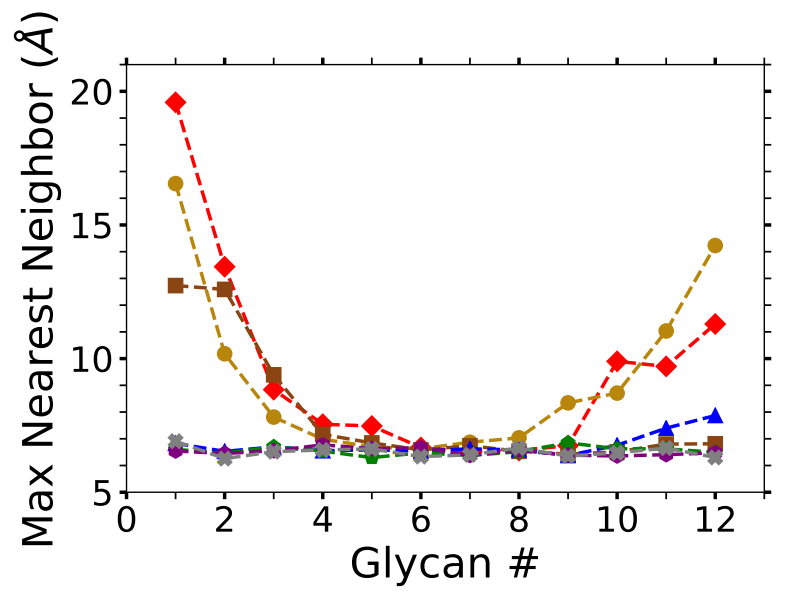

(d) $400 \mathrm{~ns}$

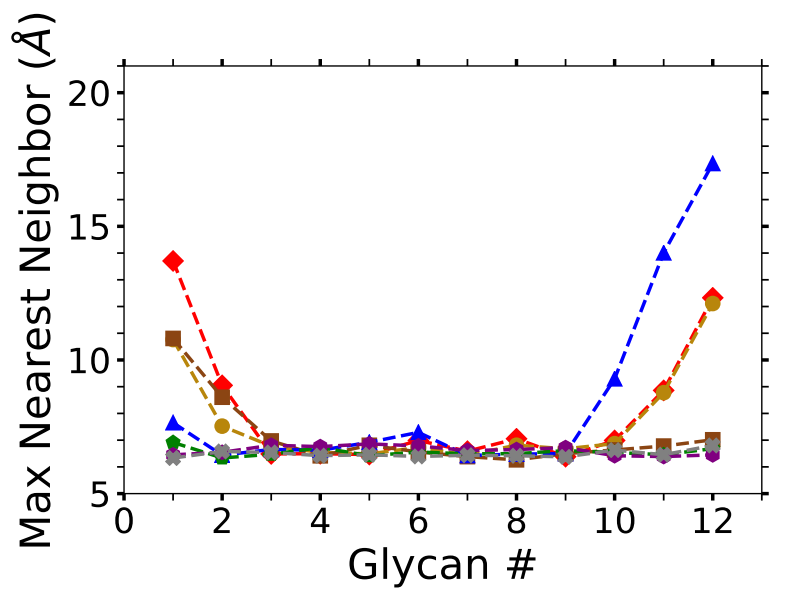

(c) $200 \mathrm{~ns}$

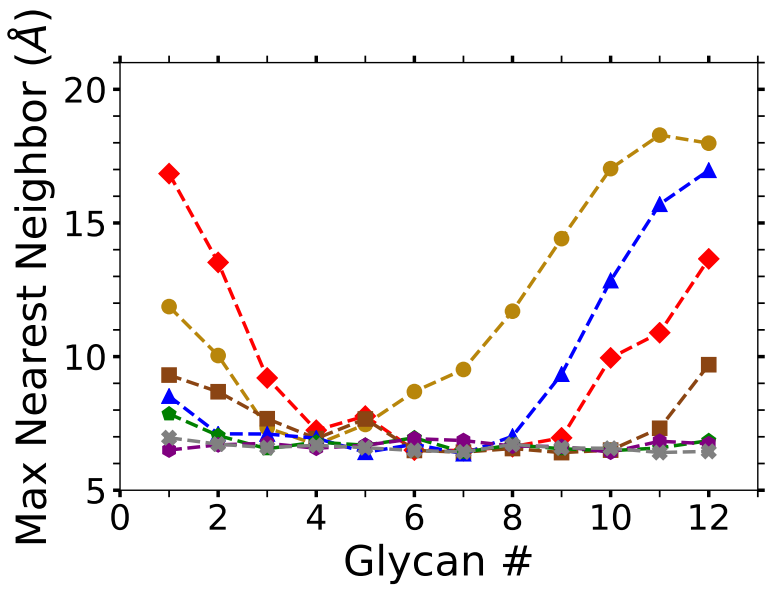

(e) $600 \mathrm{~ns}$

Figure (3.23) Maximum cellulose strand separation of the small cellulose bundles. ${ }^{22}$ 


\subsection{Hydrogen bonding}

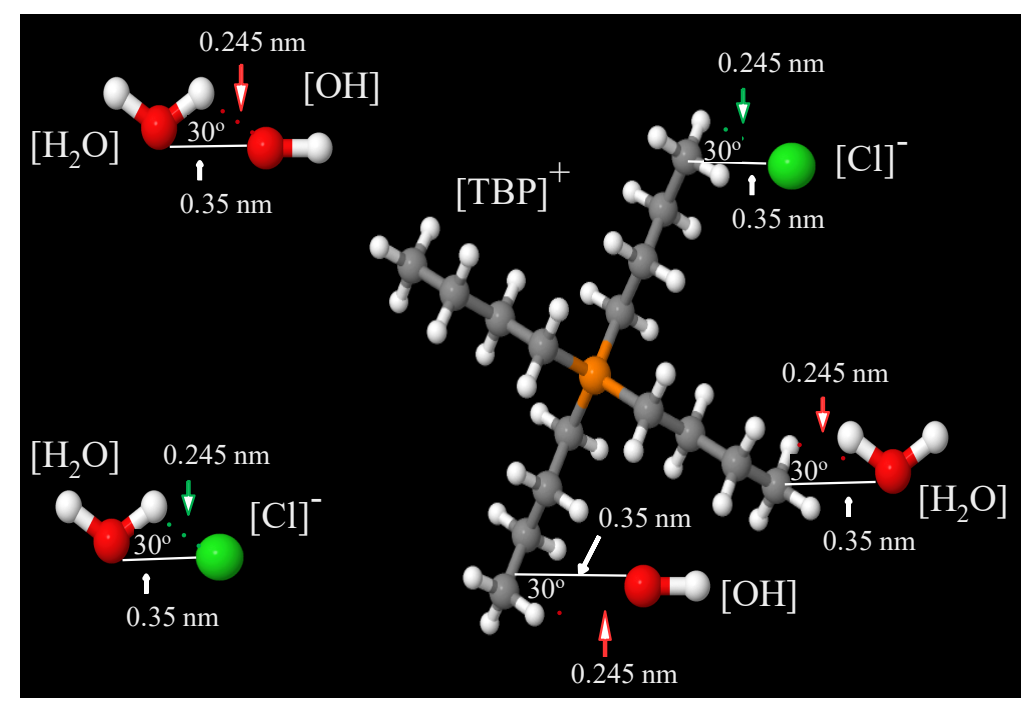

Figure (3.24) Geometric specifications for hydrogen bonds. ${ }^{58,88}$

In these simulations, a hydrogen bond is present if the hydrogen to hydrogen acceptor distance is less than or equal to $2.45 \AA$, with the hydrogen-donor-acceptor angle being less than or equal to $30^{\circ}$ (see Figure 3.24). ${ }^{60-68}$ The above requirement constrains the donor-acceptor distance to be less than or equal to $3.5 \AA,{ }^{60-68}$ as shown in Figure 3.24 .

\subsubsection{Hydrogen bonding: Only the TBPH/TBPCl-Water Solutions}

The simulations for the TBPH-water and TBPCl-water solutions were each used to calculate the hydrogen bonding between the cation-water, anion-water, water-water, and cationanion pairs (see Figure 3.25).

The anion-water hydrogen bonds are the most dominant, primarily occurring in the cellulose dissolution region (see Figure 3.25b). Additionally, the $\mathrm{OH}$-water hydrogen bonding around 99 to $99.97 \mathrm{~mol} \%$ water is in agreement with Sun et al., who showed approximately $5.1 \mathrm{OH}$-water hydrogen bonds per hydroxide when the hydroxide was infinitely diluted in water. ${ }^{89}$ On the opposite side of the spectrum, the cation-anion hydrogen bonding has the lowest number of hydrogen bonds when compared to the other hydrogen bonding types in the 70 to $99.97 \mathrm{~mol} \%$ water concentration range. In Figure 3.25d, the cation-anion hydrogen bonding decays in an approximately linear fashion as the water concentration increases, 


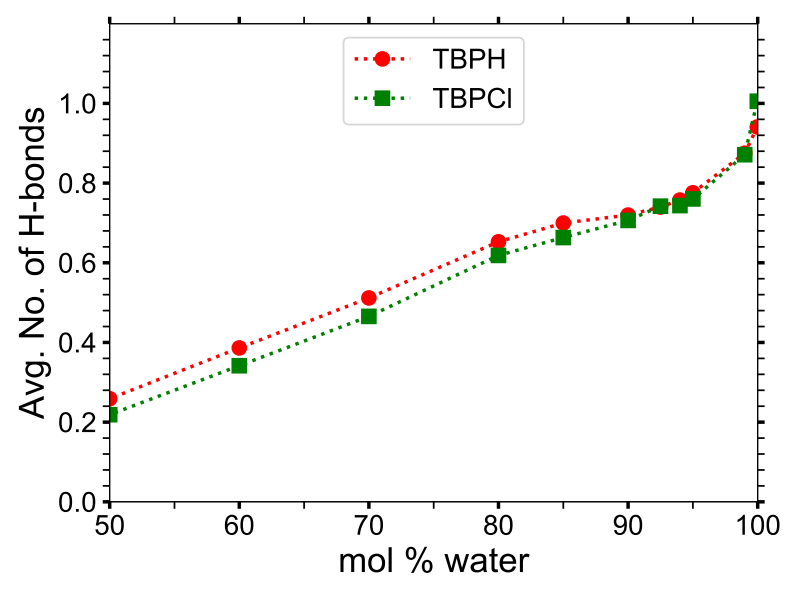

(a) cation-water pairs

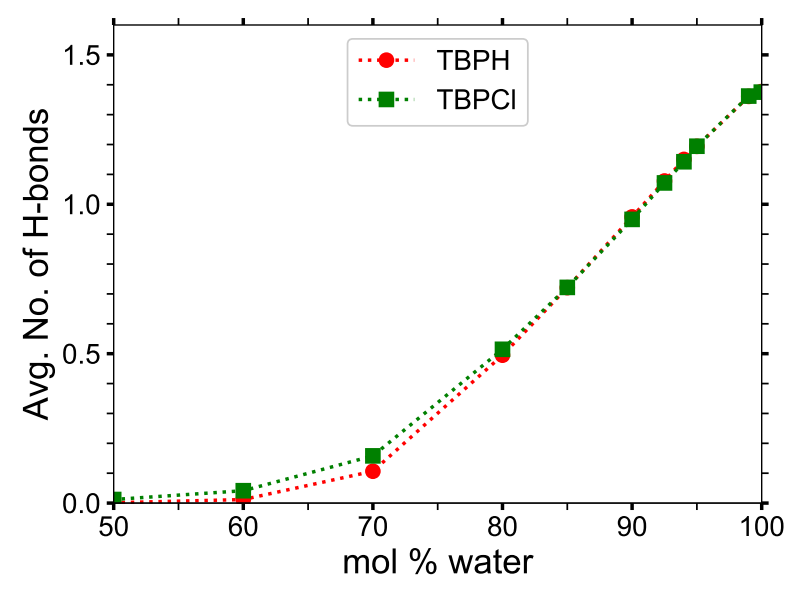

(c) water-water pairs

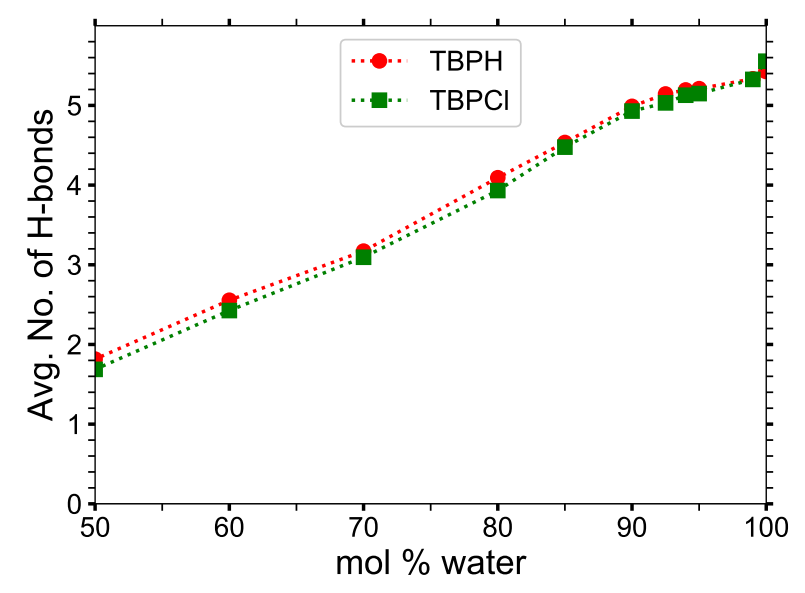

(b) anion-water pairs

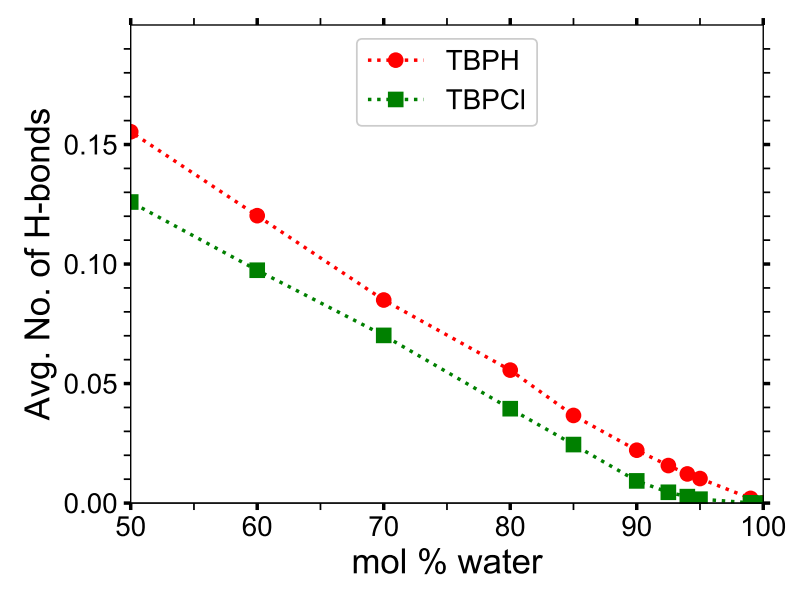

(d) cation-anion pairs

Figure (3.25) Average hydrogen bonds at $300 \mathrm{~K}$. The first part of the labeling represents the per molecule basis (i.e., $\mathrm{x}-\mathrm{y}$ represents the average number of hydrogen bonds between $\mathrm{x}$ and $\mathrm{y}$ per molecule of $\mathrm{x}) .{ }^{58}$ 
which is attributed to the anion being more attracted to the water. As expected, the waterwater hydrogen bonding follows a similar trend to the water clustering data (see Figure $3.25 \mathrm{c})$.

Figure 3.25a shows the cation-water pairs plateau between 85 and 94 mol \% water, which is the region where the water veins are present. This plateau is believed to occur due to the near-constant contact area between the cation and water in the water vein formation range. Once out of the water vein region and into higher water concentrations, the cation-water hydrogen bonds appear to increase exponentially.

\subsubsection{Hydrogen Bonding Lifetimes in the TBPCl-water and Cellulose Solutions}

This work refers to the hydrogen bond lifetimes in the following notation: A $\cdots$ H-D (hydrogen Acceptor atom ...Hydrogen atom-hydrogen Donor atom). The hydrogen and hydrogen donor, H-D, are in the same molecule and share a covalent bond. The corresponding A $\cdots H-$ $\mathrm{D}$ atomic labeling is provided in Figures 2.1 and 2.2. For example, the atoms in the TBP molecules are as follows: CT's are the carbons; HP and HC's are the hydrogens; P is the phosphorus. The most critical hydrogen bonding lifetimes are those between the chloridecellulose hydroxyl hydrogens ( $\mathrm{Cl} \cdots \mathrm{HO} 2-\mathrm{O} 2$ and $\mathrm{Cl} \cdots \mathrm{HO} 3-\mathrm{O} 3)$. When the chloride-cellulose hydroxyl hydrogen lifetimes fall below the cellulose's largest intra-strand hydrogen bonding

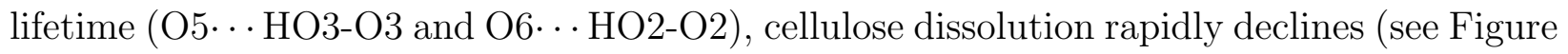
$3.26)$.

The hydrogen bonding lifetimes were calculated between the Cl-bundle, TBP-bundle, water-bundle, bundle-bundle, Cl-CT, Cl-water, and CT-water pairs (see Tables 3.3, 3.4, $7.12,7.13$, and 7.14). The data contain high variations due to the limited time snapshots from which the lifetimes were obtained. Therefore, it is essential to look at the average values and trends, not single points. Not every possible combination of hydrogen bonding lifetimes was analyzed, so the analysis and comparisons are solely based on the analyzed hydrogen bonding lifetimes, which for the study were determined to be the most significant. The significance was qualitatively determined by analyzing most of the probable combinations and removing the potential hydrogen bonding lifetimes that would be very short. This qualitative method estimated the hydrogen bonds based on their atomic charges but is prone to human errors, 
so there could be other hydrogen bonding lifetimes that play a role in the dissolution of cellulose in the TBPCl-water solution.

The anion-cellulose carbons (Cl..H's-C's) have relatively average hydrogen bonding lifetimes across all concentrations $(0.490$ to $1.13 \mathrm{ps})$, as seen in Table 3.3. The anionscellulose hydroxyl oxygen pairs, $\mathrm{Cl}$-. HOx's-Ox's, have the largest solvent-cellulose hydrogen bonding lifetime, and are listed in order of decreasing strength: $\mathrm{Cl} \cdots \mathrm{HO} 2-\mathrm{O} 2, \mathrm{Cl} \cdots \mathrm{HO} 3-$ O3, and $\mathrm{Cl} \cdots \mathrm{HO}$-O6 , for which all decay in strength with increasing water concentrations. The hydrogen bonding lifetimes across all concentrations for the $\mathrm{Cl} \cdots \mathrm{HO} 2-\mathrm{O} 2, \mathrm{Cl} \cdots \mathrm{HO} 3-$ O3, and Cl-.HO6-O6 pairs last for 48.9 to $>31445 \mathrm{ps}, 8.20$ to $>20848 \mathrm{ps}$, and 11.3 to 7189 ps, respectively. These Cl ‥HOx's-Ox's hydrogen bonds last many orders of magnitude longer than any other hydrogen bonding pairs, except the inter cellulose hydrogen bond between $\mathrm{O} 4 \cdots \mathrm{H} 2-\mathrm{C} 2$ (see Table 7.13), allowing the chloride to greatly interrupt the cellulose

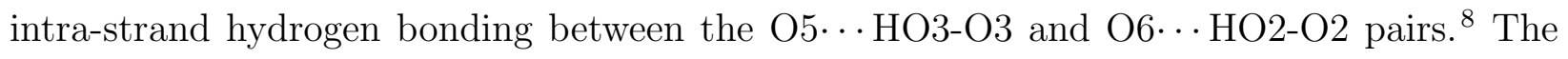
O5 $\cdots$ HO3-O3 and O6 $\cdots$ HO2-O2 hydrogen bonding lifetimes are 2.88 to 18.8 ps and 62.6 to 3318 ps, respectively (see Table 3.4).

The $\mathrm{Cl} \cdots \mathrm{HO} 2-\mathrm{O} 2$ and $\mathrm{Cl} \cdots \mathrm{HO} 3-\mathrm{O} 3$ have greater hydrogen bonding lifetimes than the intra-strand hydrogen bonding pairs (O5 $\cdots$ HO3-O3 and O6 $\cdots$ HO2-O2) between 63.1 to $79.4 \mathrm{~mol} \%$ water. However, they start to move below intra-strand lifetimes between 79.4 to $86.8 \mathrm{~mol} \%$ water, where the cellulose dissolution power of the solution begins to weaken dramatically (see Figure 3.26). Once the $\mathrm{Cl} \cdots \mathrm{HO} 2-\mathrm{O} 2$ and $\mathrm{Cl} \cdots \mathrm{HO} 3-\mathrm{O} 3$ are very weak and fairly stable, around 12 to 104 ps, the solution is no longer capable of dissolving cellulose. The anion $\cdots$ HO2-O2 and anion . . HO3-O3 lifetimes, or similar intra-strand hydrogen bond disrupters, could be a parameter programmed into the simulations to calculate where its falls below the intra-strand bonding lifetimes, thereby, indicating where the cellulose dissolution power of the IL co-solvent may begin to decline and vanish.

The Cl...HOx's-Ox's hydrogen bond's high strength helps in disrupting the intra-strand and inter-strand hydrogen bonds. ${ }^{15}$ The bonding of the cellulose strands to chloride, or similar anions, appears to loosen them from the bundle and assist in their dissolution. ${ }^{8,15,78}$ The most drastic disruption of all the hydrogen bond lifetimes with increasing water concentrations occurs for the Cl..HOx's-Ox's pairs (see Figure 3.26). These hydrogen bond 
lifetimes drop approximately 1.5 to 2.3 orders of magnitude from 63.1 to 86.8 mol \% water, and finally dropping another 0.7 to 1.1 orders of magnitude from 86.8 to 93.9 mol \% water. The $\mathrm{Cl} \cdots \mathrm{Hw}-\mathrm{Ow}$ hydrogen bond lifetimes also decay rapidly from 21.0 to $2.62 \mathrm{ps}$ with the concentrations changing from 63.1 to $86.8 \mathrm{~mol} \%$ water, as the chloride's first solvation shell becomes saturated. ${ }^{58}$ The rapid decay of the $\mathrm{Cl} \cdots \mathrm{HO} 2-\mathrm{O} 2, \mathrm{Cl} \cdots \mathrm{HO} 3-\mathrm{O} 3, \mathrm{Cl} \cdots \mathrm{HO} 6-\mathrm{O} 6$, and $\mathrm{Cl} \cdots \mathrm{Hw}-\mathrm{Ow}$ hydrogen bonding strength throughout the increasing water concentrations is presumably a large factor in the ability to dissolve cellulose. However, this anionic loosing of cellulose strands is by no means the only contributing factor to the cellulose dissolution.

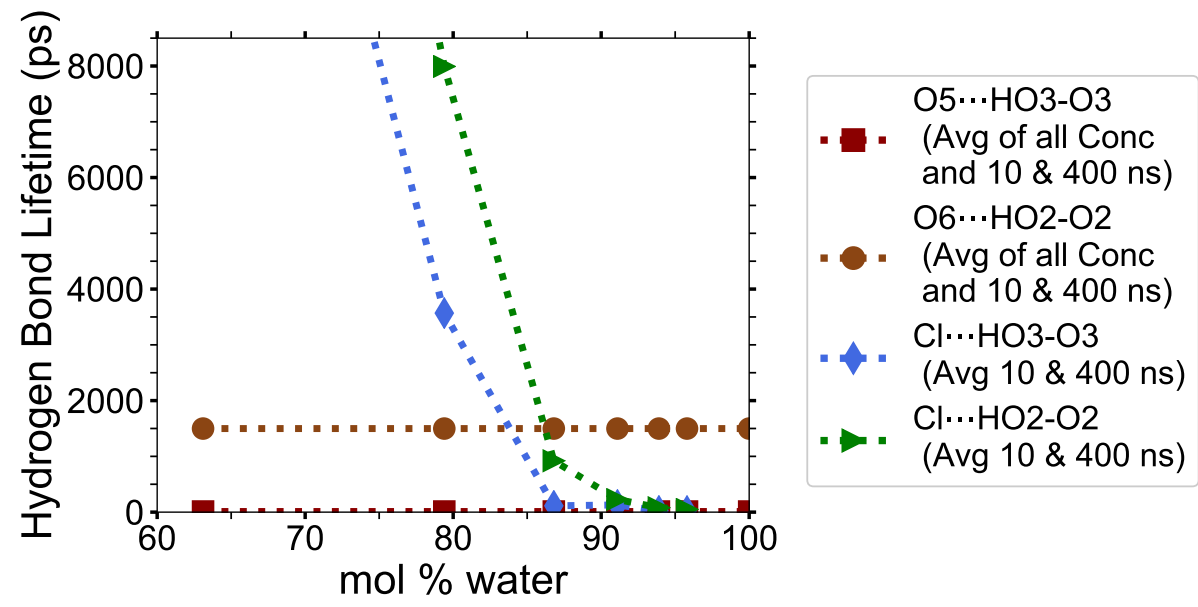

Figure (3.26) The chloride's intra-strand hydrogen bond breaking lifetimes vs. the cellulose's intra-strand hydrogen bonding lifetimes. ${ }^{22}$ The cellulose's intra-strand hydrogen

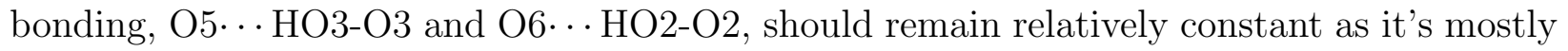
undissolved. Therefore, the intra-strand hydrogen bonding were averaged for all the concentrations and sample times (10 and $400 \mathrm{~ns}$ ). The chloride-cellulose hydroxyl oxygen's hydrogen bonds, $\mathrm{Cl} \cdots \mathrm{HO} 3-\mathrm{O} 3$ and $\mathrm{Cl} \cdots \mathrm{HO} 2-\mathrm{O} 2$, were averaged for the 10 and $400 \mathrm{~ns}$ times to yield less time-correlated data. ${ }^{22}$

The TBP molecules have the least number of hydrogen bonds with the peeling cellulose strand, except for the $63.1 \mathrm{~mol} \%$ water concentration, where there is not much water. The CT...HO's-O's, Ox's...H's-CT's hydrogen bonding lifetimes are rather small in comparison to the $\mathrm{Cl}$ - . HOx's-Ox's hydrogen bonding lifetimes (0.076 to 0.236 ps vs. 6.78 to > 31445 ps). The CT...H's-C's hydrogen bonding lifetimes are approximately equal to the Cl *. H's-C's lifetimes, which shows that there is little hydrogen bonding preference between them $(0.285$ to $0.427 \mathrm{ps}$ vs. 0.490 to $>1.13 \mathrm{ps}$ ). The CT‥-H's-C's hydrogen bonds are only stronger 
than some of the cellulose-cellulose hydrogen bonds. By comparing the hydrogen bonding, pairwise energies and the simulation snapshots, it appears that TBP does participate in the cellulose strand separation, at least in part by hydrogen bonding. Based on the hydrogen bond lifetimes, the TBP can hydrogen bond to the cellulose strands for short durations. The Ox's...H's-CT's hydrogen bonds are all about the same strength, with shrinking hydrogen bonding lifetimes for increasing water concentrations. Since the Ox's $\cdots$ H's-CT's hydrogen bonds are approximately the same strength, the cellulose oxygens all participate equally in hydrogen bonding, provided there are no steric hinderances. The CT $\cdots$ Hw-Ow hydrogen bonding lifetimes are very weak (0.026 to $0.038 \mathrm{ps})$, indicating long-term hydrogen bonding is less probable.

The water molecules have steady and moderate hydrogen bonding lifetimes with the peeling cellulose strands. Specifically, the $\mathrm{Ow} \cdots \mathrm{HO} 2-\mathrm{O} 2$ and $\mathrm{Ow} \cdot \mathrm{HO}$-O6 hydrogen bonding lifetimes are the highest for water, producing moderate strengths of 1.05 to $11.3 \mathrm{ps}$ and 0.789 to $12.1 \mathrm{ps}$, respectively. Water's Ow $\cdots \mathrm{HO} 2-\mathrm{O} 2$ and $\mathrm{Ow} \cdot \mathrm{HO} 6-\mathrm{O} 6$ hydrogen bonding strengths also decrease with increasing water concentration, dropping approximately 0.5 orders of magnitude from 79.4 to $93.9 \mathrm{~mol} \%$ water. The water hydrogen bonding strengths are

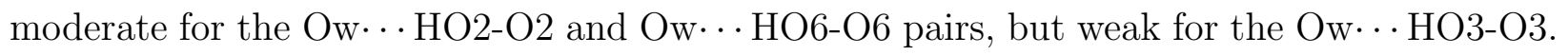
The $\mathrm{Ow}$... HO3-O3 hydrogen bonding lifetimes do not appear to decay with increasing water concentration, falling between 0.026 to $2.58 \mathrm{ps}$, with the variance or range of values occurring from random sampling with no notable trend. The water hydrogen bonding strengths suggest that the water can assist in the cellulose strand separation in the Ow.. HO2-O2 and $\mathrm{Ow} \cdot \mathrm{HO}$-O6 6 pairs, but the chloride anion is largely required for the Ow$\cdot \cdot \mathrm{HO} 3-\mathrm{O} 3$ separation. While water interrupts the cellulose's hydrogen bonding, it lacks the enhanced duration and ability to maintain multiple strong hydrogen bonds with the cellulose, as the anions do. The water and anion bonding strength to the cellulose's carbons are about the same (Cl...H's-C's and Ow...H's-C's), indicating these hydrogen bonding locations are not a factor to the anion's dissolution power.

The cellulose-cellulose hydrogen bonding lifetimes are also provided in Table 3.4, and in the Appendix (see Tables 7.12, 7.13, and 7.14). The intra-strand bonding is much stronger for the $\mathrm{O} 6 \cdots \mathrm{HO} 2-\mathrm{O} 2$ pair than the $\mathrm{O} 5 \cdots \mathrm{HO} 3-\mathrm{O} 3$. There are many inter-strand hydro- 
gen bonding lifetimes provided in these data, ranging from weak to very strong. Many of the hydrogen bonding lifetimes decay with an increasing co-solvent (not including the cellulose-cellulose hydrogen bonding lifetimes) in agreement with the tetrabutylammonium acetate (TBAAc) dimethyl sulfoxide (DMSO) simulations, which used a central atom in each molecule to determine the molecular contact lifetimes. ${ }^{78}$ The critical evaluation for any IL co-solvent solution is determined by the co-solvent type and the 'working concentration range' where the solution can effectively dissolve cellulose. For the TBPCl-water solution, the 'working range' for water would ideally be at a higher concentration to absorb some of the water naturally contained in the biomass. Therefore, a balance likely exists in many IL water solutions where the declining anion...HOx's-Ox's hydrogen bond's strength must be balanced with a higher water concentration and other properties that affect cellulose dissolution (i.e., increased diffusion). 
Table (3.3) TBPCl hydrogen bonding lifetimes (ps), Part 1 of 5

\begin{tabular}{|c|c|c|c|c|c|c|c|}
\hline \multirow[b]{2}{*}{$\mathrm{A} \cdots \mathrm{H}-\mathrm{D}^{a}$} & \multicolumn{7}{|c|}{ mol \% water } \\
\hline & 63.1 & 79.4 & 86.8 & 91.1 & 93.9 & 95.8 & 100 \\
\hline Cl $\cdots \mathrm{H}^{\prime} \mathrm{s}-\mathrm{C}^{\prime} \mathrm{s}^{b}(10 \mathrm{~ns})$ & 1.06 & 0.683 & 0.592 & 0.598 & 0.365 & 0.728 & -- \\
\hline $\mathrm{Cl} \cdots \mathrm{H}$ 's-C's ${ }^{b}$ (400 ns) & 0.543 & 0.654 & 1.13 & 0.802 & 0.644 & 0.490 & -- \\
\hline Cl*. H's-CT's ${ }^{b, d}(10 \mathrm{~ns})$ & 0.158 & 0.152 & 0.147 & 0.147 & 0.144 & 0.143 & -- \\
\hline $\mathrm{Cl} \cdots \mathrm{H}^{\prime} \mathrm{s}-\mathrm{CT} \mathrm{s}^{b, d}$ (400 ns) & 0.160 & 0.151 & 0.146 & 0.148 & 0.140 & 0.145 & -- \\
\hline $\mathrm{Cl} \cdots \mathrm{HO} 2-\mathrm{O} 2(10 \mathrm{~ns})$ & 27,329 & 7,370 & 658 & 109 & 54.3 & 48.9 & -- \\
\hline $\mathrm{Cl} \cdots \mathrm{HO} 2-\mathrm{O} 2$ (400 ns) & $>31,445$ & 8,615 & 1,184 & 346 & 104 & 51.6 & -- \\
\hline Cl $\cdots$ HO3-O3 (10 ns) & 19,816 & 1,197 & 147 & 55.2 & 11.7 & 31.0 & -- \\
\hline $\mathrm{Cl} \cdots \mathrm{HO} 3-\mathrm{O} 3(400 \mathrm{~ns})$ & $>20,848$ & $>5,941$ & 80.2 & 203 & 32.6 & 8.20 & -- \\
\hline Cl*HHO6-O6 (10 ns) & 7,189 & 422 & 63.4 & 52.3 & 9.33 & 6.78 & -- \\
\hline $\mathrm{Cl} \cdots$ HO6-O6 (400 ns) & 6,487 & 366 & 45.5 & 9.78 & 13.4 & 11.3 & -- \\
\hline $\mathrm{Cl} \cdots \mathrm{Hw}-\mathrm{Ow}(10 \mathrm{~ns})$ & 21.0 & 7.04 & 2.62 & 2.05 & 1.77 & 1.65 & -- \\
\hline $\mathrm{Cl} \cdots \mathrm{Hw}-\mathrm{Ow}(400 \mathrm{~ns})$ & 20.8 & 7.10 & 4.14 & 2.05 & 1.77 & 1.67 & -- \\
\hline CT $\cdots \mathrm{H}^{\prime} \mathrm{s}-\mathrm{C}^{\prime} \mathrm{s}^{b}(10 \mathrm{~ns})$ & 0.396 & 0.375 & 0.361 & 0.320 & 0.285 & 0.297 & -- \\
\hline CT $\cdots \mathrm{H}^{\prime} \mathrm{s}-\mathrm{C}$ 's ${ }^{b}$ (400 ns) & 0.427 & 0.374 & 0.316 & 0.326 & 0.328 & 0.297 & -- \\
\hline CT $\cdots \mathrm{HO}^{\prime} \mathrm{s}-\mathrm{O}^{\prime} \mathrm{s}^{b}$ (10 ns) & 0.076 & 0.089 & 0.150 & 0.119 & 0.158 & 0.123 & -- \\
\hline CT $\cdots \mathrm{HO}^{\prime} \mathrm{s}-\mathrm{O}^{\prime} \mathrm{s}^{b}$ (400 ns) & 0.138 & 0.072 & 0.137 & 0.103 & 0.080 & 0.093 & -- \\
\hline $\mathrm{CT} \cdot \cdots \mathrm{Hw}-\mathrm{Ow}(10 \mathrm{~ns})$ & 0.038 & 0.033 & 0.030 & 0.029 & 0.027 & 0.026 & -- \\
\hline $\mathrm{CT} \cdots \mathrm{Hw}-\mathrm{Ow}(400 \mathrm{~ns})$ & 0.037 & 0.033 & 0.030 & 0.028 & 0.028 & 0.026 & -- \\
\hline
\end{tabular}

${ }^{a} \mathrm{~A} \cdots \mathrm{H}-\mathrm{D}=$ hydrogen Acceptor atom $\cdots$ Hydrogen atom-hydrogen Donor atom. ${ }^{22}$

Note: (H-D) share a covalent bond. Data was started at 10.2 and $400.2 \mathrm{~ns}$.

${ }^{b}$ Averaged data

${ }^{c} \mathrm{NA}$ means no hydrogen bonds found at the start of these calculations.

${ }^{d}$ The H's are the HP and HC2, HC3, and HC4 atoms in the TBP molecule.

${ }^{f}$ MDAnalysis H-bond lifetimes do not work with $>100,000$ atoms in the analysis.

For lifetimes without auto-correlation values of zero (i.e., values with $>$ symbol), the final auto-correlation values are $<0.05$ unless otherwise noted: ${ }^{g}<0.1 ;^{h}<0.15$. 
Table (3.4) TBPCl hydrogen bonding lifetimes (ps), Part 2 of 5

\begin{tabular}{|c|c|c|c|c|c|c|c|}
\hline \multirow[b]{2}{*}{$\mathrm{A} \cdots \mathrm{H}-\mathrm{D}^{a}$} & \multicolumn{7}{|c|}{ mol \% water } \\
\hline & 63.1 & 79.4 & 86.8 & 91.1 & 93.9 & 95.8 & 100 \\
\hline Ow. $\cdots H^{\prime}{ }^{\prime}-C ' s^{b}(10 \mathrm{~ns})$ & 0.723 & 0.693 & 0.370 & 0.464 & 0.405 & 0.394 & 0.325 \\
\hline Ow $\cdots \mathrm{H}^{\prime} \mathrm{s}-\mathrm{C}^{\prime} \mathrm{s}^{b}$ (400 ns) & $\mathrm{NA}^{c}$ & 0.451 & 0.621 & 0.468 & 0.380 & 0.419 & 0.325 \\
\hline Ow $\cdots \mathrm{H}^{\prime} \mathrm{s}-\mathrm{CT} \mathrm{s}^{\mathrm{s}}{ }^{b, d}(10 \mathrm{~ns})$ & 0.159 & 0.155 & 0.154 & 0.150 & 0.148 & 0.148 & -- \\
\hline Ow $\cdots \mathrm{H}^{\prime} \mathrm{s}-\mathrm{CT} \mathrm{s}^{\prime} \mathrm{s}^{b}$ (400 ns) & 0.159 & 0.158 & 0.154 & 0.152 & 0.149 & 0.146 & -- \\
\hline Ow $\cdots \mathrm{HO} 2-\mathrm{O} 2(10 \mathrm{~ns})$ & 11.3 & 8.08 & 4.92 & 4.12 & 1.94 & 2.87 & 1.10 \\
\hline Ow. $\cdots$ HO2-O2 (400 ns) & $\mathrm{NA}^{c}$ & 1.79 & 6.00 & 2.72 & 3.40 & 2.52 & 1.05 \\
\hline Ow $\cdots$ HO3-O3 $(10 \mathrm{~ns}$ & 0.026 & 1.08 & 0.584 & 0.917 & 0.747 & 0.749 & 0.596 \\
\hline $\mathrm{Ow} \cdot \cdots \mathrm{HO} 3$ & $\mathrm{NA}^{c}$ & $\mathrm{NA}^{c}$ & 0.617 & 0.731 & 1.23 & 2.58 & 0.611 \\
\hline $\mathrm{Ow} \cdot \cdots \mathrm{HO}$ & 6.63 & 12.1 & 4.19 & 3.03 & 3.20 & 2.34 & 0.792 \\
\hline $\mathrm{Ow} \cdots \mathrm{HOG}$ & $\mathrm{NA}^{c}$ & 5.02 & 3.81 & 3.28 & 3.64 & 2.80 & 0.789 \\
\hline $\mathrm{O} 2 \cdots \mathrm{H}$ 's- & 0.207 & 0.173 & 0.196 & 0.210 & 0.180 & 0.175 & -- \\
\hline $\mathrm{O} 2 \cdot \cdots \mathrm{H}^{\prime} \mathrm{s}-\mathrm{CT}{ }^{\prime}$ & 0.202 & 0.199 & 0.194 & 0.185 & 0.199 & 0.176 & -- \\
\hline $\mathrm{O} 3 \cdots \mathrm{H}$ 's- & 0.210 & 0.177 & 0.171 & 0.186 & 0.215 & 0.181 & -- \\
\hline 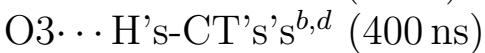 & 0.215 & 0.191 & 0.178 & 0.164 & 0.181 & 0.181 & -- \\
\hline O6* *H's-CT's s,d (10 ns) & 0.206 & 0.210 & 0.190 & 0.207 & 0.176 & 0.186 & -- \\
\hline O6* $\cdots \mathrm{H}^{\prime} \mathrm{s}-\mathrm{CT} \mathrm{s}^{b, d}$ (400 ns) & 0.220 & 0.203 & 0.194 & 0.195 & 0.197 & 0.189 & -- \\
\hline O4 $\cdots \mathrm{H}^{\prime} \mathrm{s}-\mathrm{CT} \mathrm{T}^{\mathrm{b}}{ }^{b, d}(10 \mathrm{~ns})$ & 0.222 & 0.204 & 0.176 & 0.199 & 0.161 & 0.181 & -- \\
\hline 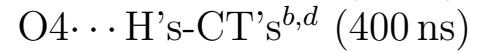 & 0.214 & 0.202 & 0.246 & 0.181 & 0.176 & 0.186 & -- \\
\hline O5 $\cdots \mathrm{H}^{\prime} \mathrm{s}-\mathrm{CT} \mathrm{s}^{b, d}(10 \mathrm{~ns})$ & 0.236 & 0.207 & 0.198 & 0.178 & 0.183 & 0.199 & -- \\
\hline O5 $\cdots \mathrm{H}^{\prime} \mathrm{s}-\mathrm{CT} \mathrm{s}^{b, d}$ (400 ns) & 0.200 & 0.223 & 0.205 & 0.207 & 0.166 & 0.179 & -- \\
\hline O5 $\cdots$ HO3-O3 (10 ns) & 15.3 & 17.4 & 17.4 & 18.8 & 16.9 & 4.54 & 3.85 \\
\hline O5 $\cdots$ HO3-O3 (400 ns) & 3.26 & 3.12 & 11.9 & 14.4 & 13.3 & 2.88 & 11.8 \\
\hline O6 $\cdots \mathrm{HO} 2-\mathrm{O} 2(10 \mathrm{~ns})$ & 3,009 & 428 & 497 & 2,423 & 2,830 & 2,327 & 588 \\
\hline $\mathrm{O} 6 \cdots \mathrm{HO} 2-\mathrm{O} 2(400 \mathrm{~ns})$ & 457 & 62.6 & 388 & 505 & 3,318 & 2,082 & 2,066 \\
\hline
\end{tabular}

${ }^{a} \mathrm{~A} \cdots \mathrm{H}-\mathrm{D}=$ hydrogen Acceptor atom $\cdots$ Hydrogen atom-hydrogen Donor atom. ${ }^{22}$

Note: (H-D) share a covalent bond. Data was started at 10.2 and $400.2 \mathrm{~ns}$.

${ }^{b}$ Averaged data

${ }^{c} \mathrm{NA}$ means no hydrogen bonds found at the start of these calculations.

${ }^{d}$ The H's are the HP and HC2, HC3, and HC4 atoms in the TBP molecule.

${ }^{f}$ MDAnalysis H-bond lifetimes do not work with $>100,000$ atoms in the analysis.

For lifetimes without auto-correlation values of zero (i.e., values with $>$ symbol), the final auto-correlation values are $<0.05$ unless otherwise noted: ${ }^{g}<0.1 ;^{h}<0.15$. 


\subsection{Pairwise Energies and Hydrogen Bonding of the Peeling Strands in TBPCl-water}

Throughout the dissolution process, the pairwise energies of the peeling cellulose strands identify the favorable (negative), unfavorable (positive), and neutral contributions from the individual molecular types in the solvent and the rest of the cellulose bundle. During the cellulose dissolution process, the pairwise energies show favorable interactions between the peeling strand and both the $\mathrm{Cl}$ and TBP molecules. The pairwise energies between the TBP and peeling strand (TBP-peeling strand) are consistently favorable and potentially net negative, which was not seen for the imidazolium-based ILs. ${ }^{15}$ Hence, this may be a critical and unique attribute that enables the TBP-based solutions to dissolve cellulose in high water concentrations.

The two yellow strands are used for the pairwise energy and hydrogen bonding comparison as they are the mirror images of one another, and hence, both strands should have the same pairwise energies given that they are at the same dissolution state in the process (see Figure 2.3). If the cellulose bundle-peeling strand pairwise energies are increasing, then that yellow cellulose strand is peeling. The pairwise energies and hydrogen bonding for the first peeling yellow strand are shown in Figures 3.27, 3.28, and 3.29. The pairwise energies and hydrogen bonding for the second peeling yellow strand are not shown in the main text but can be found in the Appendix (see Figures 7.25, 7.26, and 7.27). Additional two-dimensional pairwise energy and hydrogen bond plots at a fixed concentration are shown in the Appendix (see Figures $7.28,7.29,7.30,7.31,7.32,7.33$, and 7.34). The TBP, Cl, and water pairwise energy values are each proportionally attributed to interaction with the peeling cellulose strand.

In the region of dissolution, 63.1 to $91.1 \mathrm{~mol} \%$ water, the chlorides - peeling strand have a net negative pairwise energy, which offsets the net positive bundle-peeling strand pairwise energy, allowing the cellulose strands separate via an energetically favorable pathway (see Figures $3.27 \mathrm{a}, 3.27 \mathrm{~b}, 7.25 \mathrm{a}$, and $7.25 \mathrm{~b}) .{ }^{15}$ There is a direct correlation between the net negative chlorides-peeling strand pairwise energies and the formation of a chloride-peeling strand hydrogen bonds, indicating that the hydrogen bonding is the primary driver of the net negative pairwise energy (see Figures $3.27 \mathrm{~b}, 3.28 \mathrm{~b}, 7.25 \mathrm{~b}$, and $7.26 \mathrm{~b}) .{ }^{15}$ The bundle - peeling 
strand net positive pairwise energies are also directly correlated to the loss of hydrogen bonds between the cellulose bundle and peeling strands. ${ }^{15}$ This effect is due to the chlorides hydrogen bonding to the cellulose strand (see Figures 3.27a, 3.28a, 7.25a, and 7.26a). ${ }^{15}$ The cellulose strand hydrogen bonding is broken down further between the intra-strand and interstrand hydrogen bonding in Figures 3.29 and 7.27. Both the intra-strand and inter-strand hydrogen bonding are significantly reduced during the strand separation process where the chloride hydrogen bonds to the peeling strand, unbinding the strand from the cellulose bundle and allowing it to twist as it peels away.

Where the TBPCl-water solution has the maximum cellulose dissolution power, the TBPpeeling strand pairwise energies are a favorable and net negative, as shown by Figures 3.27c and 7.25c. The large fluctuations in the TBP-separating strand pairwise energies over time, most dramatically seen at $63.1 \mathrm{~mol} \%$ water, indicate an increasingly negative pairwise energy, followed by a molecular relaxation after the cellulose separates (also see Figure 7.28). The TBP-peeling strand hydrogen bonds are loosely correlated to the net negative pairwise energies, demonstrating that dispersion or Coulombic forces also drive the net negative pairwise energies and the cellulose separation process (see Figures 3.27c, 3.28c, 7.25c and 7.26c). The $63.1 \mathrm{~mol} \%$ water simulation shows a very noticeable net negative pairwise energy during the separation process, while all the other concentrations are approximately neutral to slightly net negative. However, these net negative energies may relax back to the net neutral favorable pairwise energies during longer simulations or with the loss of the hydrogen bonding, similar to other studies. ${ }^{15}$ While other studies suggested the hydrophobic or electrostatic forces of the TBP-peeling strand may allow the cellulose dissolution, this study quantitatively shows the hydrogen bonding, dispersion and Coulombic forces energetically assisting the cellulose dissolution, especially in non-alkali solutions. ${ }^{78,90-92}$

For much of the I- $\alpha$ and I- $\beta$ cellulose bundle dissolution in ethyl-3-methylimidazolium acetate (EMIM-Ac), the EMIM-peeling strand has an unfavorable pairwise energy, and the Ac-peeling strand maintains a favorable pairwise energy. ${ }^{15}$ For the I- $\alpha$ cellulose bundle, the EMIM-peeling strand and Ac-peeling strand pairwise energies very quickly relax to a net neutral energy contribution after each part of the dissolution, which was not witnessed in this study, although, this is a different type of cellulose bundle. ${ }^{15}$ In this study, the chloride- 
peeling strand net negative and the bundle-peeling strand net positive energy interaction are similar to the I- $\beta$ cellulose bundle dissolution with the EMIM-Ac IL, shown by Rabideau et $a l .{ }^{15}$ However, the TBP-peeling strands appear to always have a favorable and increasingly negative pairwise energy, instead of the mostly unfavorable EMIM-peeling strand pairwise energy. ${ }^{15}$ Therefore, the slightly net negative and favorable contribution of the TBP-peeling strand pairwise energy could be a unique attribute of the TBPCl-water mixture and the TBP family of ILs.

The water-peeling strand pairwise energy can be slightly net positive, net negative, or net neutral, and is partially determined by the current state of the water-peeling strand hydrogen bonding (see Figures 3.27d, 7.25d, 7.28, 7.29, 7.30, 7.31, 7.32, 7.33, and 7.34). The water-peeling strand pairwise energy can either be favorable or unfavorable at the lower water concentrations, which is dependent on the current state of the cellulose strand separation and the water-peeling strand hydrogen bonding. However, water appears to assist in cellulose dissolution as it can weakly hydrogen bond to the cellulose strand, providing short-term and slightly lower pairwise energies (see Tables 3.3 and 3.4 for the hydrogen bonding lifetimes). At moderate water concentrations, these water properties may help prevent cellulose strand reformation by allowing water to get between the cellulose strands. Therefore, the approximately net energy neutral and somewhat lower short-term pairwise energies, could be a factor in its success in preventing cellulose strand reformation. 


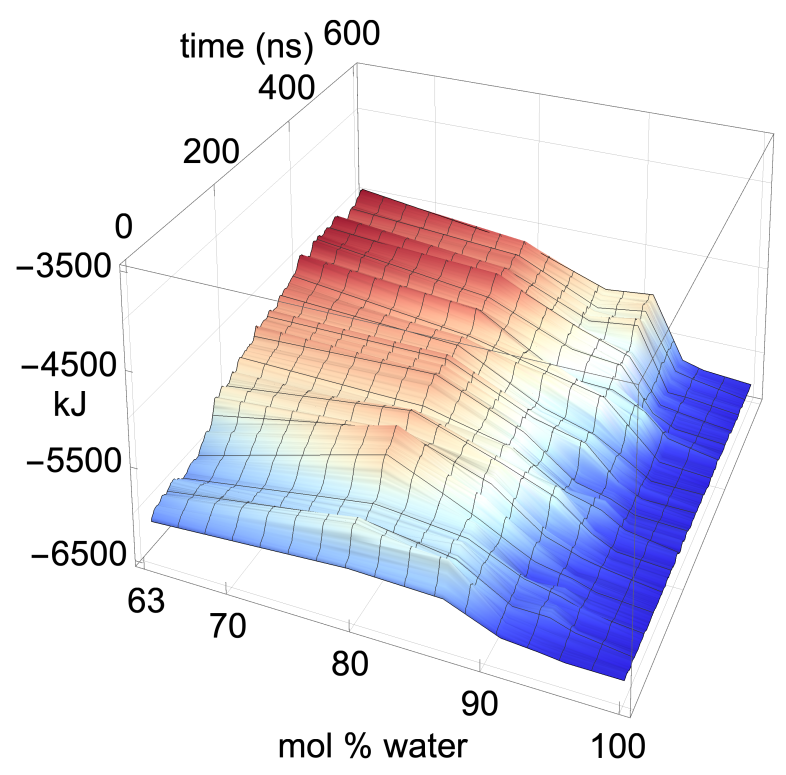

(a) (Bundle and peeling strand)-peeling strand

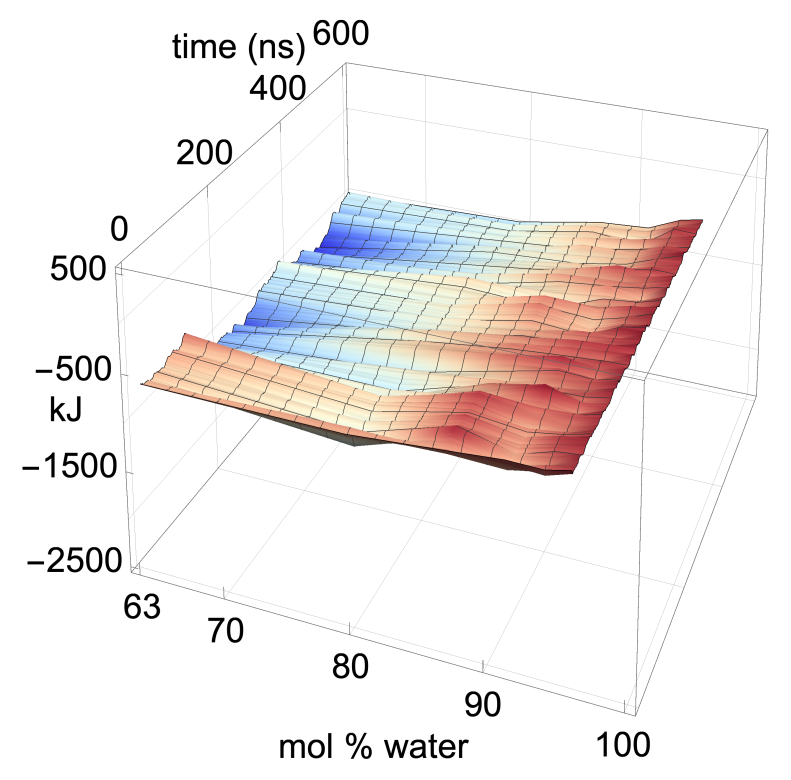

(c) TBP-peeling strand

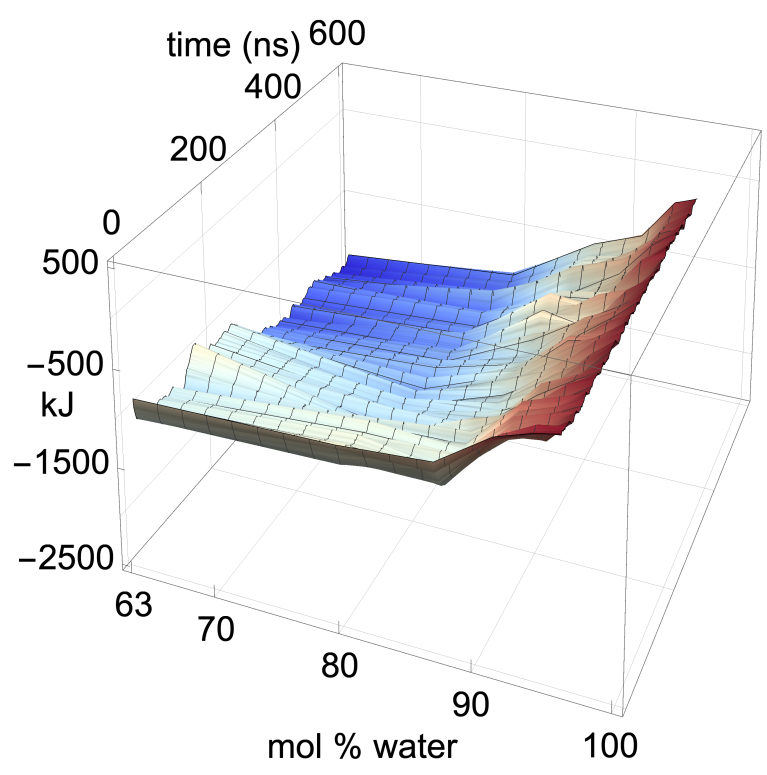

(b) Cl-peeling strand

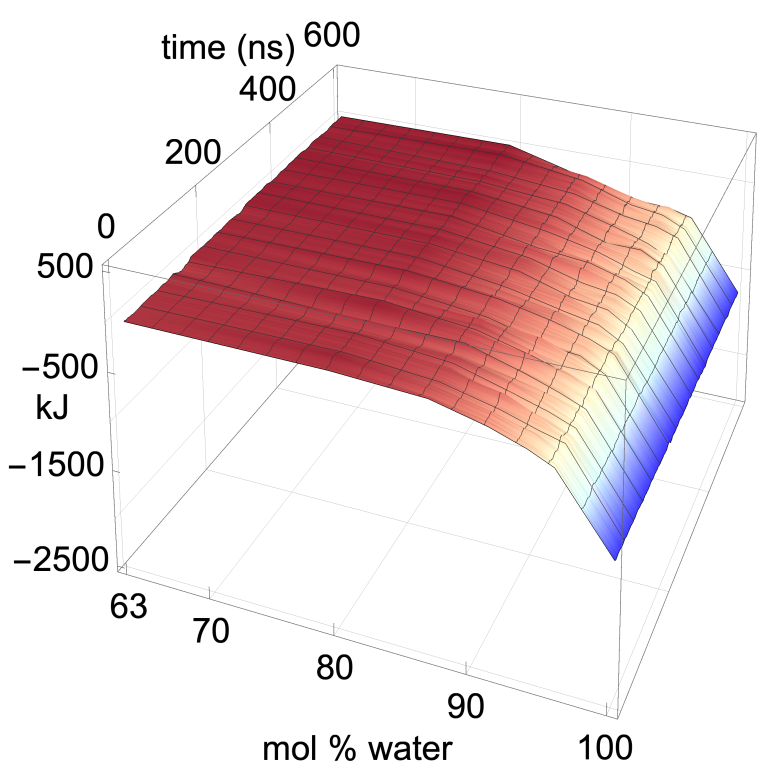

(d) Water-peeling strand

Figure (3.27) The pairwise energies for the first yellow separating strand at $360 \mathrm{~K}$. The pairwise energies are shown between the following: (a) (bundle-peeling strand and peeling strand)-peeling strand (i.e., within the strand and with the rest of the cellulose bundle); (b) Cl-peeling strand; (c) TBP-peeling strand; (d) Water-peeling strand. These data represent the first yellow strand to peel or the non-peeling yellow strand if no yellow strands peeled in the simulation. The data were averaged over 1000 data points, using a rolling average. Every $100^{\text {th }}$ point was then plotted to maintain plot clarity. ${ }^{22,23}$ 


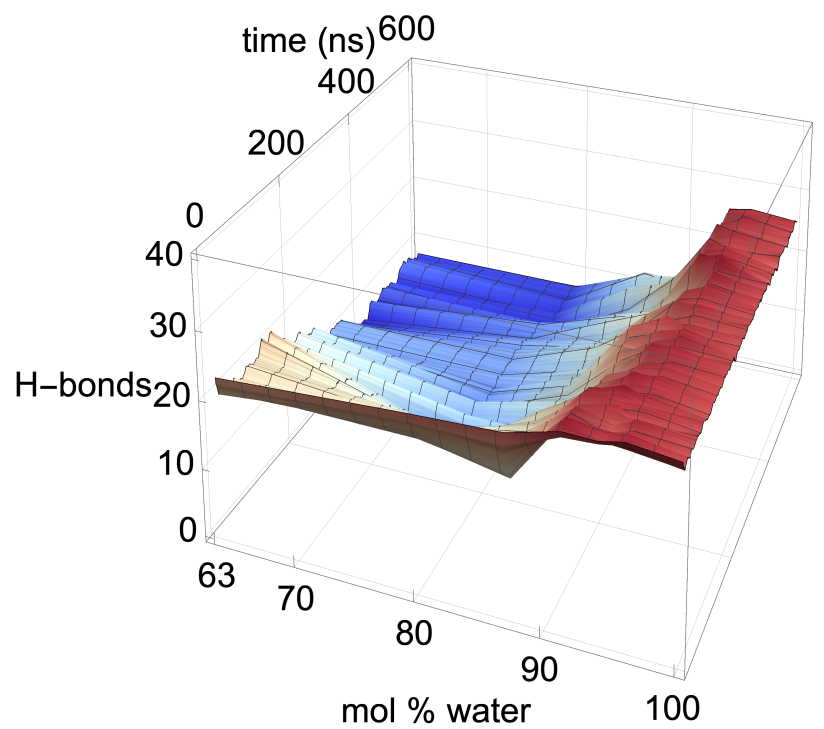

(a) Peeling strand: intra-strand and inter-strand

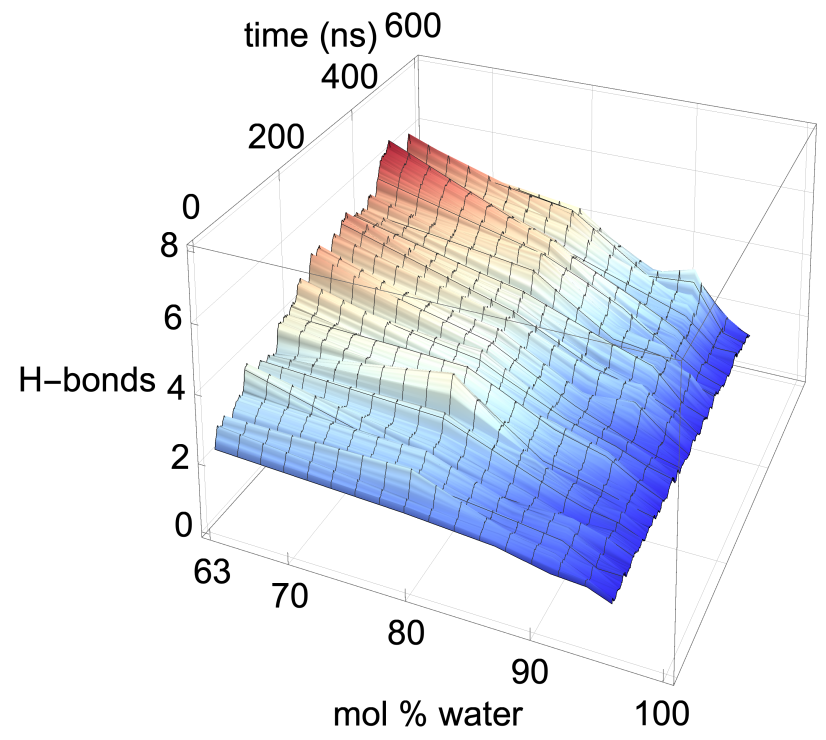

(c) TBP-peeling strand

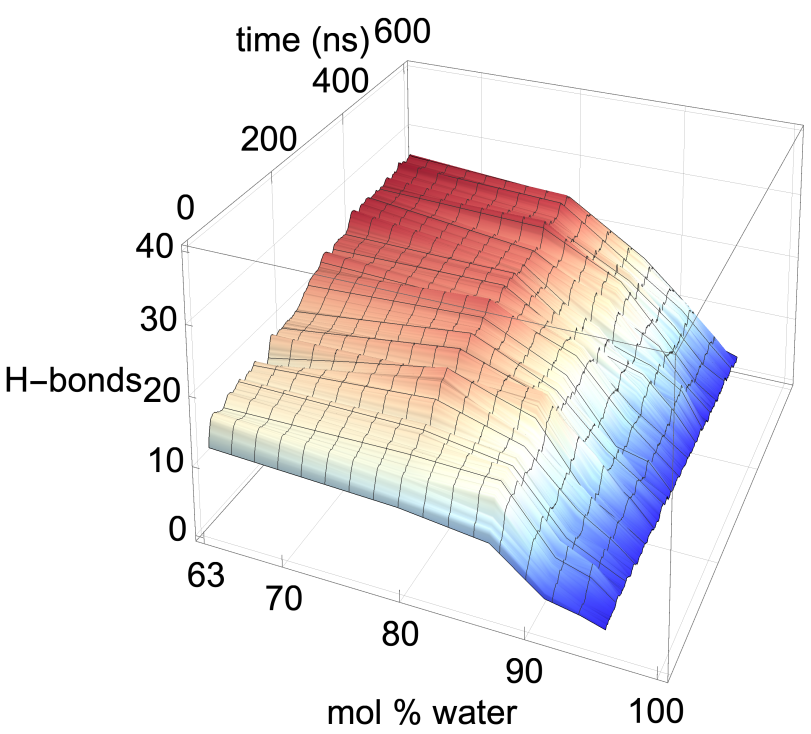

(b) Cl-peeling strand

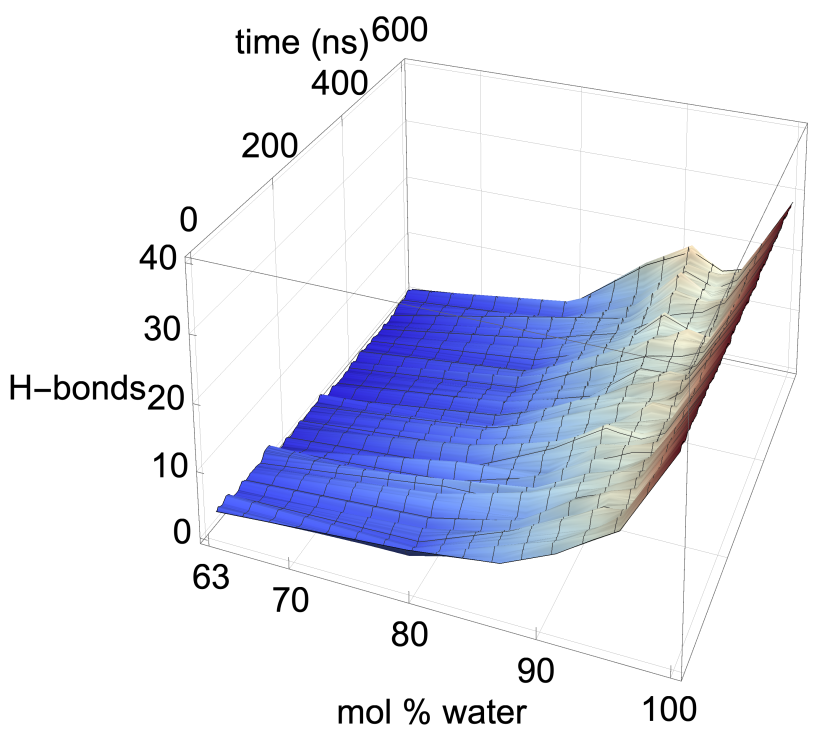

(d) Water-peeling strand

Figure (3.28) The hydrogen bonding for the first yellow separating strand at $360 \mathrm{~K}$ (Part 1 of 2). The hydrogen bonds are shown between the following: (a) (bundle-peeling strand and peeling strand)-peeling strand (i.e., intra-strand and inter-strand hydrogen bonds for the separating strand); (b) Cl-peeling strand; (c) TBP-peeling strand; (d) Water-peeling strand. These data represent the first yellow strand to peel or the non-peeling yellow strand if no yellow strands peeled in the simulation. The data were averaged over 1000 data points, using a rolling average. Every $100^{t h}$ point was then plotted to maintain plot clarity. ${ }^{22,23}$ 


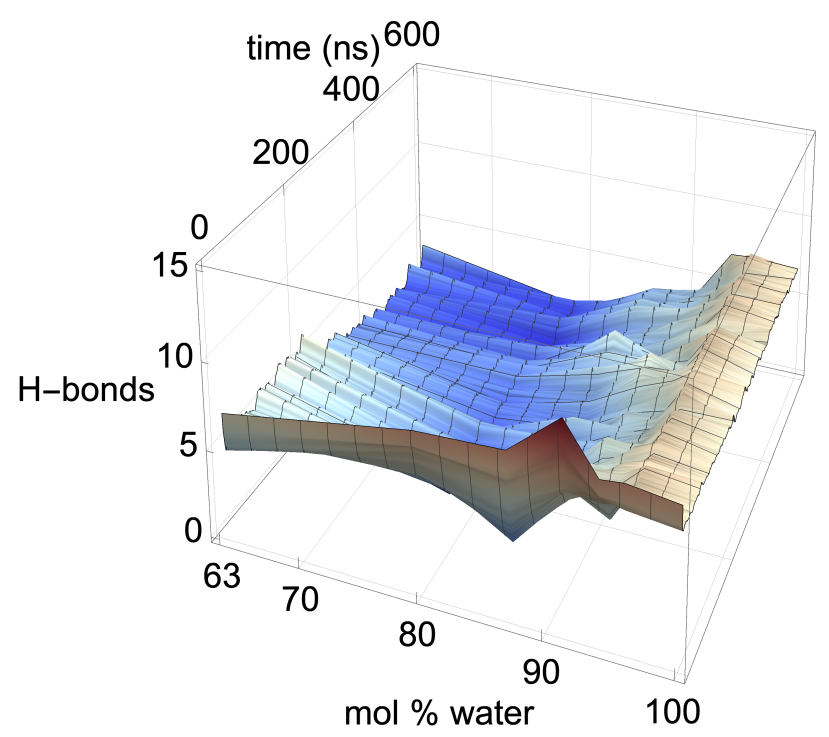

(a) Peeling strand: intra-strand

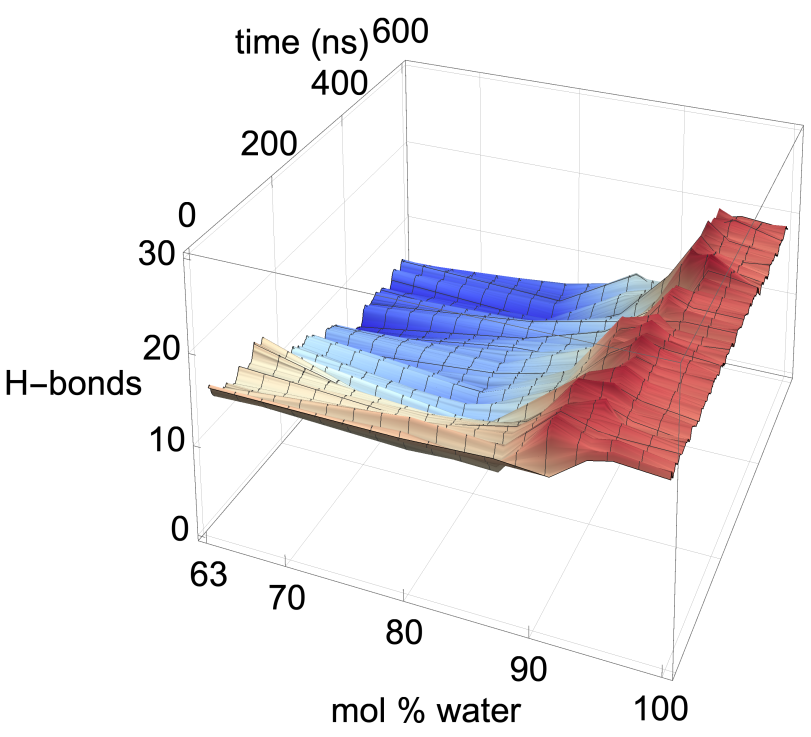

(b) Peeling strand: inter-strand

Figure (3.29) The hydrogen bonding for the first yellow separating strand at $360 \mathrm{~K}$ (Part 2 of 2). The hydrogen bonds are shown between the following: (a) Cellulose: intra-peeling strand (i.e., the intra-strand hydrogen bonds for the separating strand); (b) Cellulose: interpeeling strand (i.e., the inter-strand hydrogen bonds for the separating strand). These data represent the first yellow strand to peel or the non-peeling yellow strand if no yellow strands peeled in the simulation. The data were averaged over 1000 data points, using a rolling average. Every $100^{\text {th }}$ point was then plotted to maintain plot clarity. ${ }^{22,23}$

\subsection{Cellulose Dissolution Mechanism in TBPCl-water}

All the concentrations that dissolve cellulose reveal the same dissolution mechanism. The first layer strands and the corner strands are the easiest to peel away from the cellulose bundle as these strands have the least hydrogen bonds stabilizing them (i.e., both intrastrand and inter-strand hydrogen bonds), and they are least sterically hindered from the rest of the cellulose bundle (see Figures 3.2 and 3.3). Additionally, the first layer strands and the corner strands are the most solvent-exposed, leading to the most probable $\mathrm{Cl}$, TBP, and water interactions (see Figures 3.2, 3.4, and 3.5). The images show the greatest cellulose dissolution at $79.4 \mathrm{~mol} \%$ water, which agrees with the data in Figures 3.22 and 3.22b. As expected, the $100 \mathrm{~mol} \%$ water simulation shows no cellulose dissolution, but does visually exhibit cellulose bundle twisting like the rest of the concentrations. ${ }^{25,26}$ The cellulose dissolution visually 
appears to be most probable at the ends of the bundles, as the dissolution starts at the ends of the strands, while the center of the strands remains connected (also see Figure 3.23). The final dissolution images for all the water concentrations at $600 \mathrm{~ns}$ can be found in the Appendix (see Figures 7.21, 7.22, 7.23, and 7.24). The molecular representations of the TBPCl-water solution in the simulation snapshots are provided in Figure 3.30.

These images selectively depict the IL and co-solvent (solvent) to show the crucial cellulose-solvent interactions. The interior cellulose strands are the light pink, white, and purple strands in Figure 2.3. The chloride anions are shown if they are within $3.5 \AA$ of the cellulose bundle. The water and TBP molecules are only shown if they are within $3.5 \AA$ of the 10 inner glycans of the interior cellulose strands. Dashed lines depict hydrogen bonding. The green dashed lines indicate the chloride-cellulose hydrogen bonds and are shown anywhere on the cellulose bundle. The light blue dashed lines indicate the water-cellulose hydrogen bonds, which are only shown in selected images if the water is within $3.5 \AA$ of the 10 inner glycans of the interior cellulose strands.

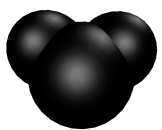

Water

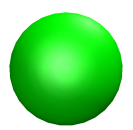

Chloride (Cl-) anion

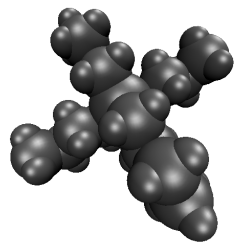

Tetrabutylphosphonium $\left(\mathrm{TBP}^{+}\right)$cation

Figure (3.30) TBPCl-water molecular representations. ${ }^{22,30}$

From a visual analysis of the simulations, the catalyzing anions initiate the strand peeling by disrupting the cellulose's hydrogen bonds, which loosen the cellulose strand (see the Pairwise Energies and Hydrogen Bonding of the Peeling Strands section and Figures 3.27, 3.28, $3.29,7.25,7.26,7.27,7.28,7.29,7.30,7.31,7.32,7.33$, and 7.34 for the quantitative analysis). The anion hydrogen bonding to the cellulose strands also provides a net negative pairwise energy, making the strand separation process energetically feasible. Most importantly, the chloride anions break the celluloses intra-strand hydrogen bonds, which provides the strand with more freedom to twist and separate (see Figures 3.29, 3.30, 3.31, 3.32, and 3.33). ${ }^{15}$ The 
variation in the dihedral angles of the peeling strands shows that the strands are twisting throughout the dissolution process in the $63.1 \mathrm{~mol} \%$ water and $91.1 \mathrm{~mol} \%$ water solutions, while the same strand in the pure water solution is not twisting (see Figure 3.33). Since the water is adequately attracted to cellulose's hydroxyl groups and the anion, it inserts between the cellulose strands, ahead of the larger TBP molecule (see Tables 3.3 and 3.4). The anions open a small pocket between the cellulose strands and remain there for a relatively long time, while the water molecules diffuse in and out of this water pocket between the cellulose strands, forming water-cellulose hydrogen bonds while in the pocket. The water molecules do insert themselves between or under the peeling strands but do not penetrate deep into the cellulose bundle. This hydrogen bonding allows the $\mathrm{Cl}$ and water to maintain the separation of the cellulose strand and makes the water less mobile, impeding strand reformation (see Figures 3.32 and 7.35). From past research, the diffusion of the TBPCl-water solution rises with increasing water concentration. ${ }^{58}$ Therefore, it is not surprising that the water molecules stay in the water pocket for longer durations at lower water concentrations due to the increased hydrogen bonding lifetimes and lower diffusion (see Figure 7.35 and Tables 3.3 and 3.4). ${ }^{58}$ The chloride anion can also form multiple hydrogen bonds within or between cellulose strands, which can also act as a barrier to cellulose strand reformation. The TBP molecule pushes its way into the existing water pocket furthering the strand separation and displacing some of the lighter water molecules. The TBP-to-water exchange ratio was determined for the $63.1 \mathrm{~mol} \%$ water and $91.1 \mathrm{~mol} \%$ water solutions by calculating the number of TBP or water molecules in the water pocket between the first peeling yellow strand and the light pink inner strand of the cellulose bundle (see Figure 7.36). The TBP to water exchange ratio (TBP:water) was approximately 3:1 and 1:2 for the $63.1 \mathrm{~mol} \%$ water and $91.1 \mathrm{~mol} \%$ water solutions, respectively. The difference in ratios is not surprising since it is nearly identical to the ratio change in the TBPCl-water concentrations. This interaction between the TBP and the peeling strand is energetically favorable (negative) with a potential net negative pairwise energy, which likely provides a thermodynamic pathway for the cellulose separation (see Figures 3.27c, 7.25c, 7.28, 7.29, 7.30, and 7.31). Once the TBP molecule can insert itself between the cellulose strands, it acts as a more stable physical barrier and cleaver to separate the cellulose. The mechanism here is strikingly similar to the cellulose bundle dissolution 
from imidazolium-based ILs, shown by Rabideau et al., ${ }^{15}$ although the imidazolium-based ILs were pure at higher temperatures without any co-solvent. ${ }^{15}$

The TBP's small tetrahedral shape is believed to play several vital roles in the ability of the TBPCl-water solution to dissolve cellulose (see Figures 3.30, 3.31, and 3.32). The first vital function of the small tetrahedral shape allows it to penetrate the small openings between the cellulose strands. The second vital function is the TBP's shape and its ability to transform into a relatively planar form and get under the cellulose strands (i.e., the lower three arms of the TBP form a relatively planar structure), in which the rotation of TBP also assists in the separation process (see Figure 3.32). The third vital function of the tetrahedral shape, quantified in our previous work, ${ }^{58}$ helps form the water vein structure, which likely influences the TBP, $\mathrm{Cl}$, and waters diffusion regime change between 80 to 92.5 mol \% water. ${ }^{58}$ The shift in the solvent's diffusion regime raises the diffusion by approximately an order of magnitude, helping all the solvent molecules to move quickly into the openings between the cellulose strands before the strands can reform, increasing the probability of further separation. ${ }^{58}$ 


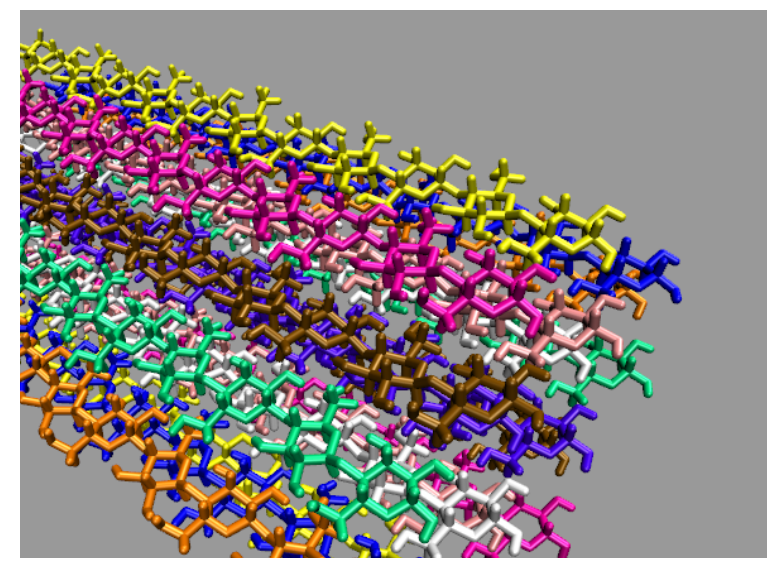

(a) $0 \mathrm{~ns}$

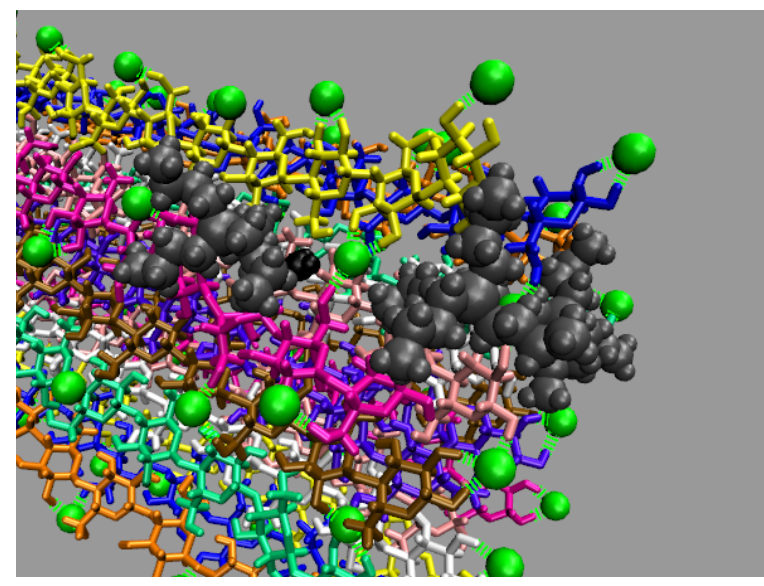

(c) $40 \mathrm{~ns}$

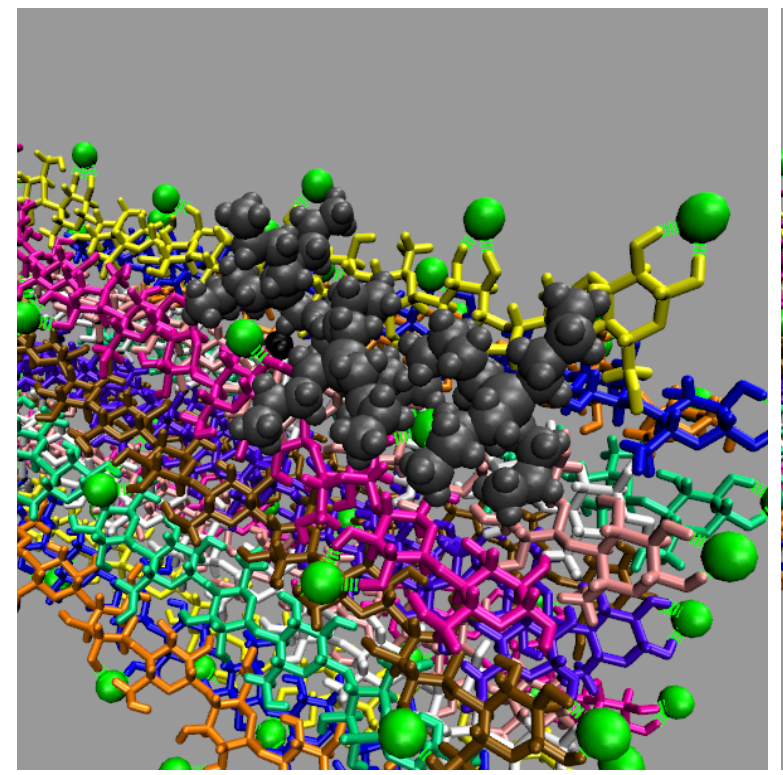

(e) $150 \mathrm{~ns}$

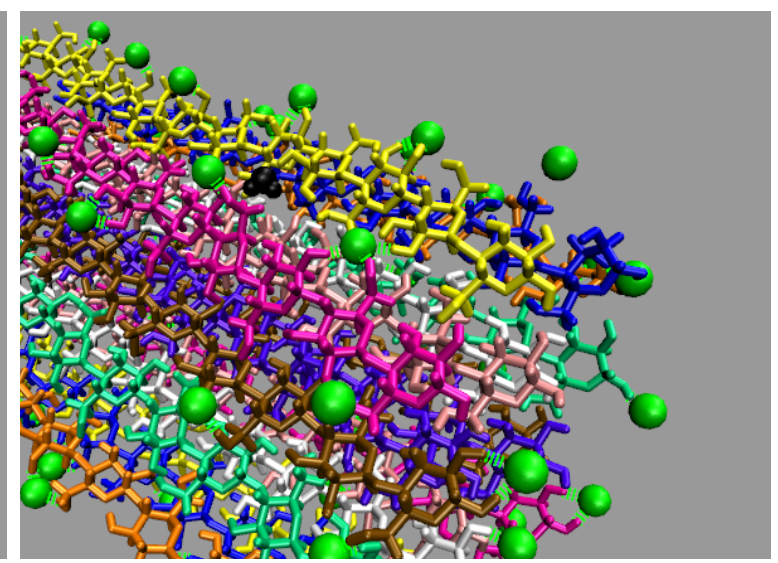

(b) $5 \mathrm{~ns}$

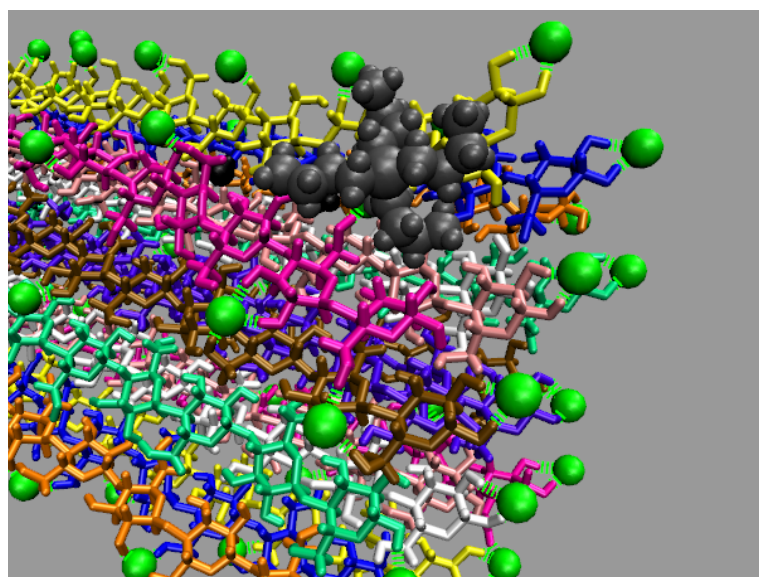

(d) $80 \mathrm{~ns}$

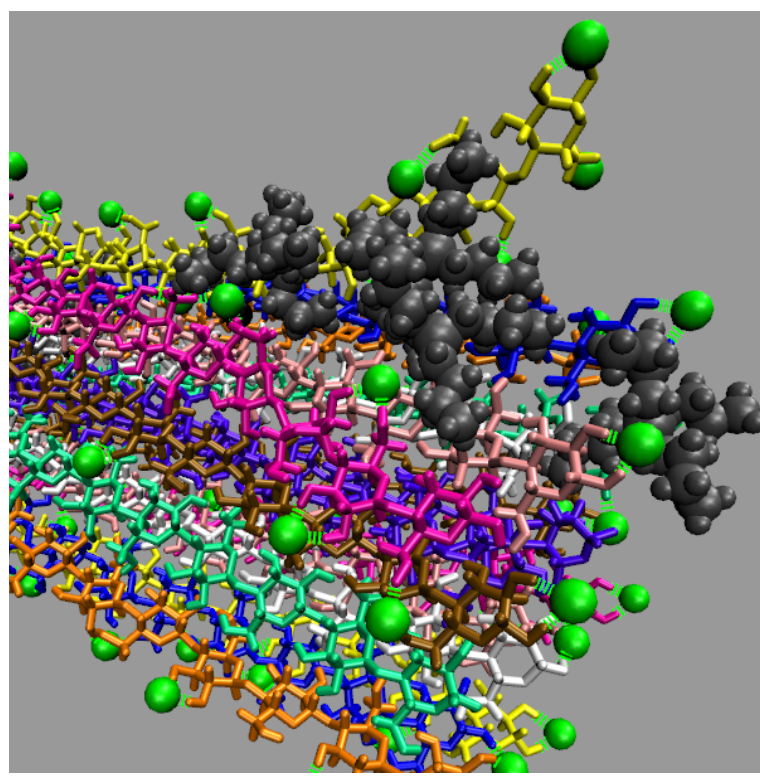

(f) $200 \mathrm{~ns}$

Figure (3.31) The cellulose dissolution process for the $63.1 \mathrm{~mol} \%$ water concentration. The green dashed lines are the Cl-cellulose hydrogen bonds. The water-cellulose hydrogen bonds are not shown in these images. ${ }^{22}$ 


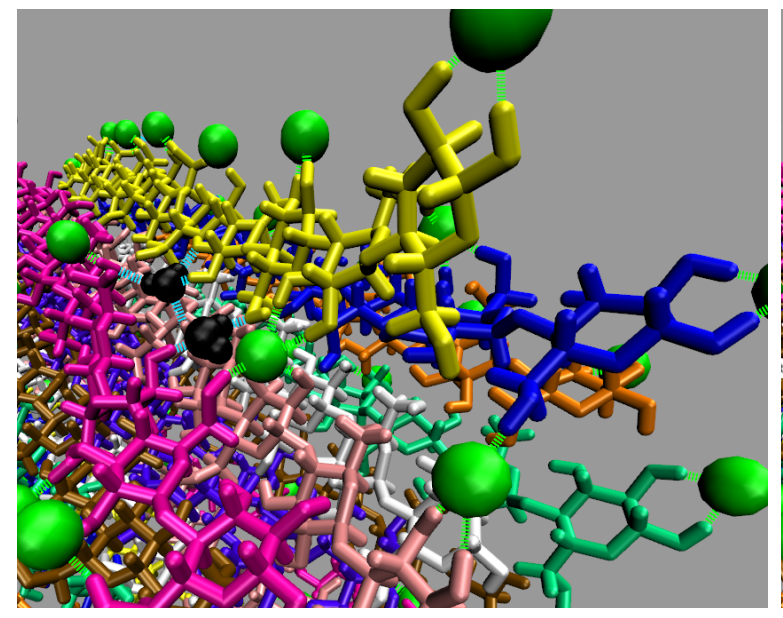

(a) $63.1 \mathrm{~mol} \%$ water at $45 \mathrm{~ns}$ (without TBP)

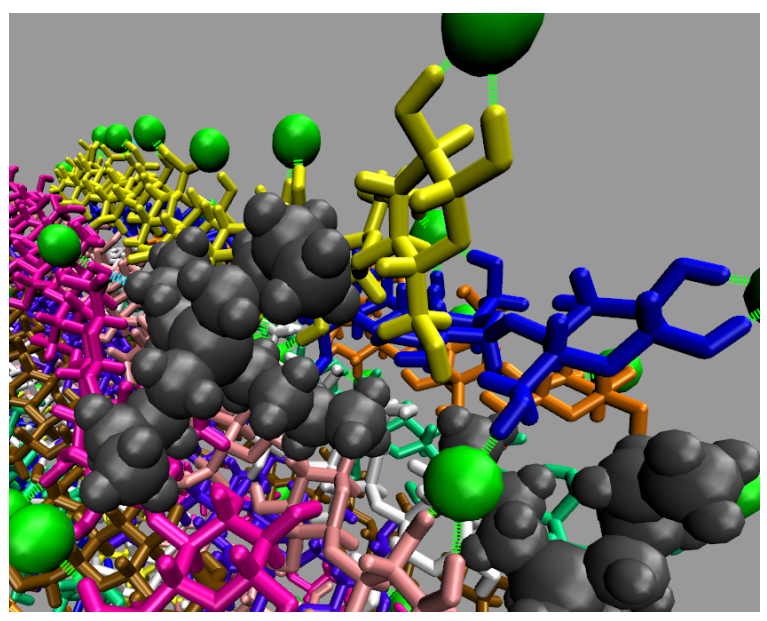

(b) $63.1 \mathrm{~mol} \%$ water at $45 \mathrm{~ns}$

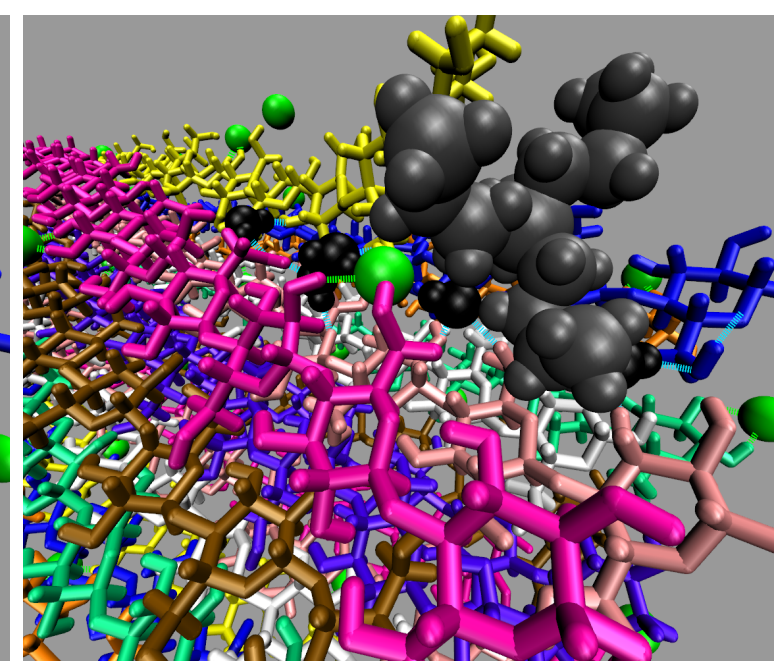

(d) $91.1 \mathrm{~mol} \%$ water at $191 \mathrm{~ns}$

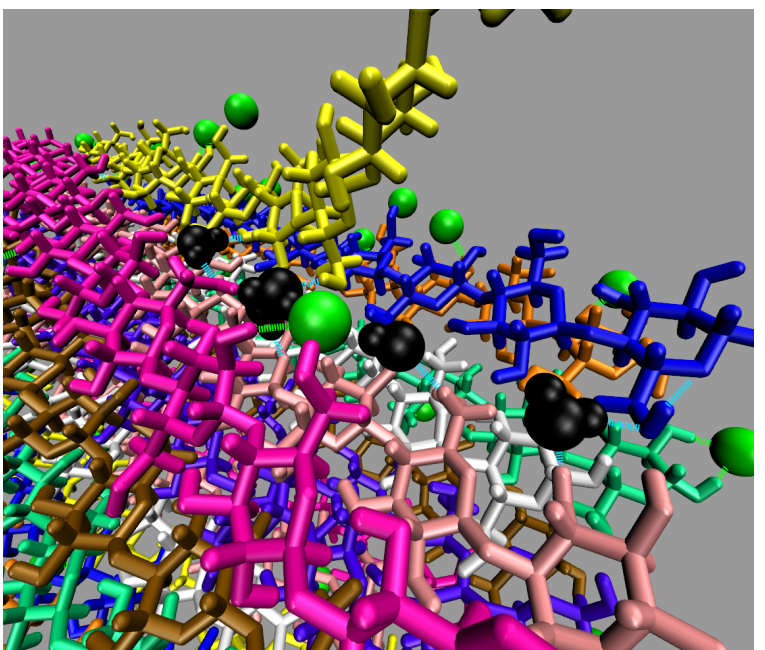

(c) $91.1 \mathrm{~mol} \%$ water at $191 \mathrm{~ns}$ (without TBP)

Figure (3.32) The cellulose dissolution process. The green dashed lines are the Cl-cellulose hydrogen bonds, while the light blue dashed lines are the water-cellulose hydrogen bonds. ${ }^{22}$ 


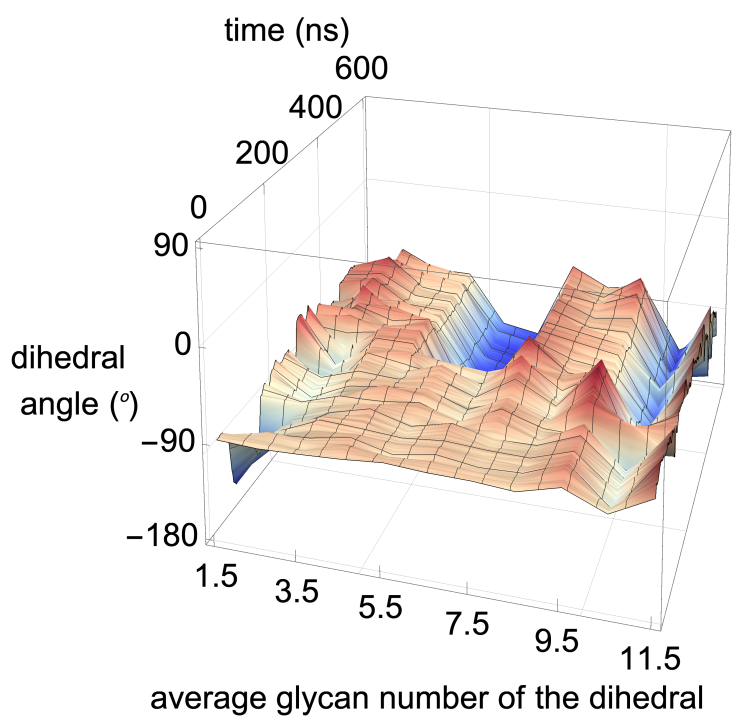

(a) 63.1 mol \% water: the first yellow peeling strand (first layer strand)

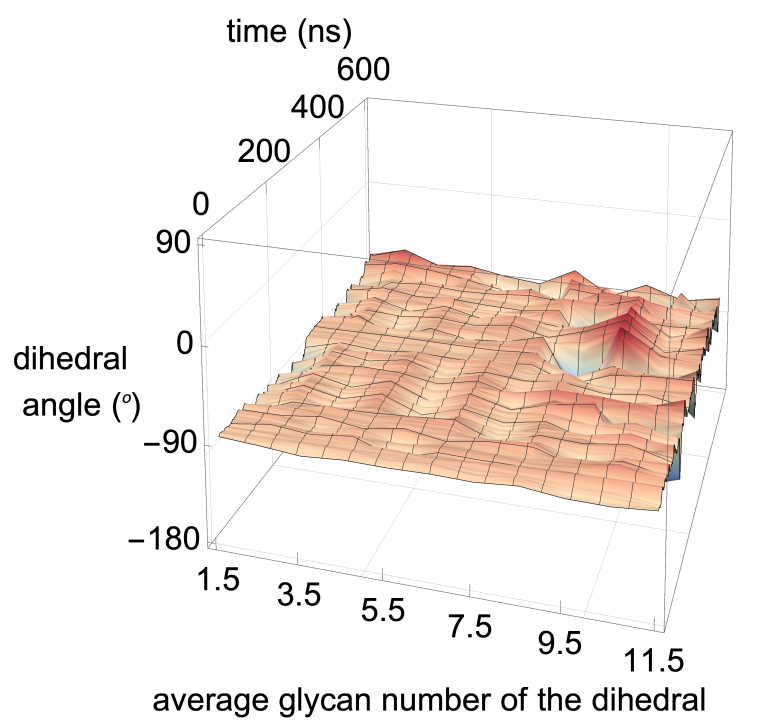

(c) $91.1 \mathrm{~mol} \%$ water: the first yellow peeling strand (first layer strand)

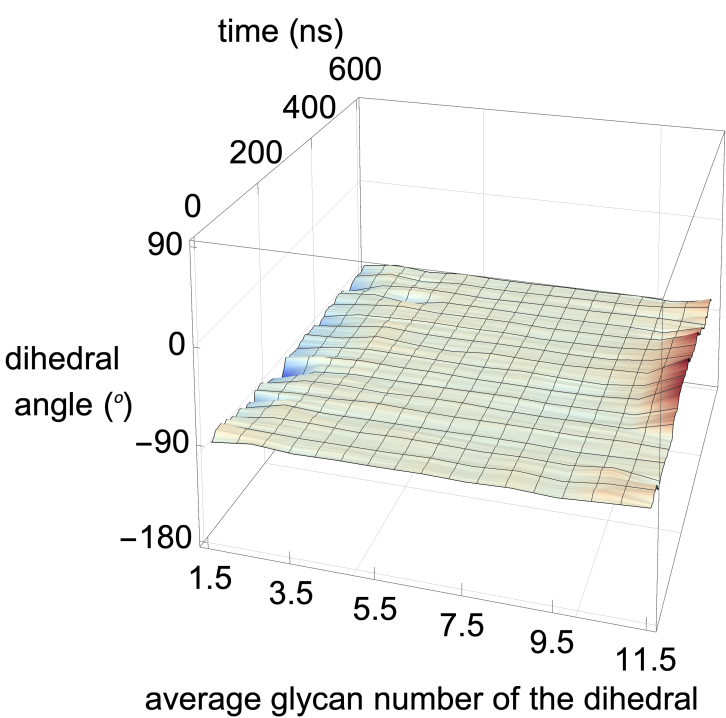

(b) 63.1 mol \% water: a non-peeling purple strand (center strand)

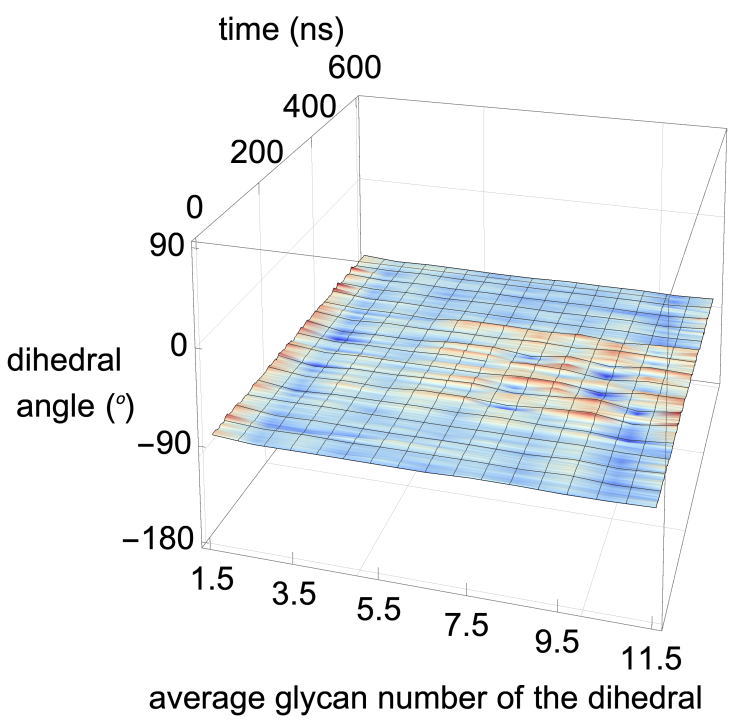

(d) 100 mol \% water: a non-peeling yellow strand (first layer strand)

Figure (3.33) The cellulose strand twisting for various concentrations at $360 \mathrm{~K}$. The cellulose strand twisting is shown between the following: (a) the first yellow peeling strand (first layer strand) at $63.1 \mathrm{~mol} \%$ water; (b) a non-peeling purple strand (center strand) at $63.1 \mathrm{~mol} \%$ water; (c) the first yellow peeling strand (first layer strand) at $91.1 \mathrm{~mol} \%$ water; (d) a non-peeling yellow strand (first layer strand) at $100 \mathrm{~mol} \%$ water. The dihedral angle is measured, in order, from the O5-C1-O4-C4 atoms, which are between two glycans (see Figure 2.1). Since the dihedral angle is between two glycans in the cellulose strand, the average glycan number is used in the plots (i.e., the dihedral angle between glycans 1 and 2 is represented as 1.5). Please see Figure 2.3 for the cellulose strand colors. The data were averaged over 1000 data points, using a rolling average. Every $100^{\text {th }}$ point was then plotted to maintain plot clarity. ${ }^{22,23}$ 


\subsection{Comparison between Alkylimidazolium IL's and TBPH / TBPCl-Water Solutions}

Alkylimidazolium-based ILs are one of the more popular and widely studied solutions for cellulose dissolution. As a result, its physical properties, and cellulose dissolving mechanisms are more established. ${ }^{1,15,62,93}$ Therefore, comparing the alkylimidazolium-based IL to the TBPH-water and TBPCl-water solutions should provide some additional insight into the unique properties TBP-based ILs.

\subsubsection{Similarities}

The water clustering profiles for the TBP-water based and alkylimidazolium-water based ILs are practically identical. ${ }^{62}$ Both water clustering profiles proliferate at $70 \mathrm{~mol} \%$ water and form a single water cluster at around $70 \mathrm{~mol} \%$ water. ${ }^{62}$ The hydrogen bonding for the waterwater pairs are practically the same in the TBP-water based and alkylimidazolium-water solutions. ${ }^{62}$ Like some TBP-water based solutions, many other IL co-solvent/anti-solvent mixtures, including the alkylimidazolium-water based solutions, show a maximum diffusivity near the pure co-solvent/anti-solvent concentration (examples include [BMIM] [Cl]-water, BMIM bis-(trifluoromethanesulfonyl)imide ([BMIM] $\left[T f_{2} N\right]$ )-methanol, [BMIM] $\left[T f_{2} N\right]$ - acetone, [BMIM] $\left[T f_{2} N\right]-1,4$-dioxane, and others). ${ }^{62,82}$

Rabideau et al. showed that the dissolution of cellulose in alkylimidazolium IL's use both the cation and anion, which played a significant role in the dissolution process. ${ }^{15}$ First, the anion breaks some of the hydrogen bonds binding a strand to the neighboring chains in the fibril. Then, the alkylimidazolium cation acts as a "wedge," effectively and permanently breaking the bonds and allowing for further dissolution. This same process seems to occur in the TBPCl-water based solutions, and potentially in other TBP-based solvents as well.

\subsubsection{Differences}

A significant physical difference between the cellulose dissolution power of the alkylimidazolium and TBPH-water solution is their operating temperature. While the alkylimidazolium ILs require moderate to high temperatures, and small amounts of water virtually eliminate 
it is cellulose dissolving power, the TBPH-water solution excels at dissolving cellulose at room temperature and with a large mole fraction of water. ${ }^{1,12,13,16,62}$ Generalizing the physical explanation, the water acts as an anti-solvent for the alkylimidazolium-based solution, ${ }^{14}$ while water acts as a co-solvent in the TBPH-water solution. ${ }^{89}$ At low to moderate temperatures, the TBPH-water based solution dissolve cellulose with high concentrations of water, and the alkylimidazolium-based ILs only work at or near the pure IL solution. From these simulations, the TBPCl-water solution also dissolves cellulose in higher water concentrations at $360 \mathrm{~K}$. However, the cellulose TBPCl-water solubility curve does not appear to have a maximum at a higher water concentration, like the TBPH-water solution. The TBPCl-water solutions cellulose solubility increasingly declines with the addition of water like the alkylimidazolium ILs; but, it appears to decrease much slower, continuing to work at higher concentrations, unlike the alkylimidazolium ILs. Water also appears to play a role in preventing the reformation of the cellulose strand by hydrogen bonding with the cellulose bundle and acting as a physical barrier to cellulose strand reformation, until the larger TBP molecule can enter and more permanently separate the cellulose strand. The role water plays here may be present in the alkylimidazolium ILs at lower water concentrations, but it is not documented in the current literature.

The diffusion regime shift could be an important factor for cellulose dissolution in the TBP-water based ILs, from 80 to $92.5 \mathrm{~mol} \%$ water, in conjunction with the water vein formation. ${ }^{62,94}$ A similar diffusion regime change may exist in the alkylimidazolium-water based solutions, but it is not documented in the current literature. ${ }^{62,83,94}$ In general, the diffusion regime shift may increase the probability for a molecule to insert itself under a cellulose strand after the anion helps loosen the hydrogen bonds that hold the cellulose strands together. ${ }^{15}$ Despite the molecule having the same probability of leaving this area, if not physically entangled or hydrogen-bonded, once the cellulose strand separates, the likelihood of the cellulose strand returning to its original position is reduced. After many iterations of separations, the cellulose strand will gradually peel away from the main cellulose bundle.

While the relative number of anion-water hydrogen bonds in the TBP-water based and alkylimidazolium-water based solutions are the same, there is one notable difference in the 
trends. ${ }^{62}$ The TBP-water based solutions have a plateau from 90 to $95 \mathrm{~mol} \%$ water, while in the alkylimidazolium-water based solutions, the anion-water hydrogen bonds continue to grow steadily with increasing water concentration. ${ }^{62}$ The anion-water plateau in the TBPwater based solutions may be an artifact of the water vein formation, allowing the solvation of the anions with water to remain relatively constant during the formation of the water veins. The TBP-water hydrogen bonds also plateau around 85 to $95 \mathrm{~mol} \%$ water, possibly due to the TBP-water surface contact area remaining fairly constant in the region of water vein formation, which could allow the TBP to retain the same interaction properties.

The chloride-peeling strand net negative and the bundle-peeling strand net positive energy interactions in the TBPCl-water simulations are analogous with the I- $\beta$ cellulose bundle dissolution with the alkylimidazolium-based IL, EMIM-Ac IL. The primary difference is the TBP-peeling strands have a favorable and increasingly negative pairwise energy, instead of the largely unfavorable EMIM-peeling strand pairwise energy. ${ }^{15}$ In the TBPCl-water simulations, the chloride-peeling strand net negative and the bundle-peeling strand net positive energy interaction is similar to the I- $\beta$ cellulose bundle dissolution with the pure EMIM-Ac IL (alkylimidazolium-based IL). ${ }^{15}$ Moreover, the TBP-peeling strands have a favorable and increasingly negative pairwise energy, rather than the mostly unfavorable EMIM-peeling strand pairwise energy. ${ }^{15}$ The slightly net negative and favorable contribution of the TBPpeeling strand pairwise energy may allow cellulose dissolution at higher water concentrations for a given temperature. 


\section{Discussion}

Since the TBPH-water and TBPCl-water solutions have the distinct ability to dissolve cellulose at low to moderate temperatures in high mol fractions of water, it is essential to understand the properties that permit this cellulose dissolution process. Understanding these properties may allow the design of different IL-water combinations that work more economically at room temperature. Knowing these properties could permit an automated search of new IL-water combinations via MD simulations coupled with a machine learning algorithm. Automating molecular dynamics (MD) simulations for the discovery of new ILs could produce superior IL-water combinations in less time than traditional experimental testing alone. Therefore, these new simulation-based search methods may be the key to designing an economically viable IL-water combination that can produce biofuels from waste biomass on an industrial scale. Other IL co-solvent/anti-solvent solutions are described below to convey the similarities, differences, current possibilities and challenges with the existing IL solvents.

The operating and capital costs of the entire process must be considered when designing an economically viable facility for converting biomass into fuel. Process separation steps can incur high costs, so the chemistry of the cellulose dissolution process should be carefully assessed. Utilizing co-solvents like DMSO and DMF can increase the process costs, as no process yields $100 \%$ recycling efficiency. The effects of the IL and co-solvent on the downstream processes should also be determined. It appears using an IL-water based solvent that can tolerate high water concentrations could be more economically feasible since water is a cheap co-solvent, already contained in the biomass, and likely less disruptive to downstream processes (i.e., enzymatic processes). There are still many process details of the IL-water or TBP-water based solvents that need to be resolved, such as separating the IL or the dissolved cellulose from the solution, and the downstream processing of the dissolved cellulose strand. Therefore, this work can not conclude that a specific co-solvent will produce the most economical solution.

1-butyl-3-methylimidazolium chloride $([\mathrm{BMIM}][\mathrm{Cl}])$ at the moderate temperatures of

$343 \mathrm{~K}$ and $373 \mathrm{~K}$ can dissolve cellulose concentrations of $3.0 \mathrm{wt} \%$ and $10 \mathrm{wt} \%$ respectively. $1,12,13$ At $363 \mathrm{~K}$ and $368 \mathrm{~K}$, a $70.8 \mathrm{~mol} \%(20 \mathrm{wt} \%)$ aqueous mixture of [BMIM][Cl] can dissolve a 
minute 1.1 wt $\%$ and 0 wt $\%$ cellulose respectively. ${ }^{1,12,13}$ This confirms that alkylimidazoliumbased ILs are excessively sensitive to the presence of water even at moderate temperatures. $^{12,13}$ To dissolve cellulose, the alkylimidazolium ILs require moderate temperatures and the absence of water, disabling their economic viability as an industrial solvent. ${ }^{62}$ While the alkylimidazolium-based ILs are effective in dissolving cellulose; they are not capable of dissolving cellulose at near room temperatures, in the presence or absence of water. ${ }^{1,12-14}$

Simulations of $[\mathrm{EMIM}][\mathrm{Ac}]$ also showed that the diffusion coefficients continually rise with increasing water concentration, ${ }^{62}$ a trend similar to that seen with the tetrabutylphosphonium hydroxide (TBPH)-water and tetrabutylphosphonium chloride ( $\mathrm{TBPCl}$ )-water mixtures and many cellulose-dissolving ILs and ILs in general. ${ }^{82}$ The alkylimidazolium-based ILs may not be the most desirable solvent for industrial processes because of their moderate to high water sensitivity and elevated operating temperatures. ${ }^{12,13}$

1-propyl-3-methylimidazolium acetate $([\mathrm{PMIM}][\mathrm{Ac}])$ and $[\mathrm{EMIM}][\mathrm{Cl}]$ show different solvation properties in mixtures, based on whether the other component is protic, such as water, or aprotic, such as DMSO. ${ }^{95,96}$ Due to hydrogen bonding with the IL co-solvent, protic co-solvents show a preferentially strong solvation potential for the anion, while aprotic co-solvents solvate the cations and anions equally. ${ }^{95}$ In the present work, the same effect is observed in the TBPH-water and TBPCl-water simulations, as the hydroxide and chloride bonds predominantly with the water co-solvent. While some researchers have found greener alternative aprotic co-solvents, ${ }^{97}$ water will usually be the most environmentally friendly and economical co-solvent.

Tetraoctylphosphonium acetate $\left(\left[\mathrm{P}_{8888}\right][\mathrm{Ac}]\right)$ with an aprotic DMSO co-solvent (40 wt $\%$ DMSO), is shown to dissolve cellulose as a function of the DMSO concentration, with a maximum cellulose solubility of $8 \mathrm{wt} \%$ cellulose. ${ }^{98}$ The cellulose can be extracted using the protic co-solvent of water, then separated using methods of liquid-liquid extraction and centrifuges with the cellulose remaining in the water and the $\left[\mathrm{P}_{8888}\right][\mathrm{Ac}]$ remaining in the DMSO, due to the longer alkyl arms. ${ }^{98-100}$ This cellulose extraction method is promising for the phosphonium based ILs, and can potentially be chemically tweaked to produce a more economically friendly solution.

Existing experimental and simulation evidence consistently shows the anions play an 
initial and critical role in breaking the cellulose's hydrogen bonds. ${ }^{15,78,101}$ Tetrabutylammonium acetate $([\mathrm{TBA}][\mathrm{Ac}])$ has been investigated in the co-solvent dimethyl sulfoxide (DMSO), showing that the acetate anions are more probable to situate themselves in locations where two hydrogen bonds form between the hydroxyl groups of the cellulose and the anion. ${ }^{78}$ The co-solvent DMSO and the TBP cations do not clearly show this dual hydrogen bonding attribute. $^{78}$ These results are in line with the findings in this TBPCl-water cellulose dissolution research, as the TBP hydrogen bonding strengths with cellulose are very small when compared with the anion.

Tetrabutylammonium acetate $([\mathrm{TBA}][\mathrm{Ac}])$ with the aprotic co-solvent DMSO, showed similar cation behavior with tetrabutylammonium hydroxide (TBAH) in a water co-solvent, both showing the TBA cation encases the dissolved cellulose. ${ }^{78}$ The mechanisms for TBA cation binding in these alkali IL and co-solvent mixtures are not in agreement. One theory suggests the hydroxide anion deprotonating the cellulose, while the other theory suggests that the hydroxide anion creates a non-bonding charge differential on the cellulose. ${ }^{78,92}$ Like other cellulose-dissolving IL solutions, the TBAH solution showed a correlation between the number of TBP molecules interacting with a single glycan unit in the cellulose strand. ${ }^{90}$ Gentile et al. reported that the interaction of the TBP cations with cellulose is critical to the cellulose dissolution process. ${ }^{90}$ It can be inferred that the TBP cation will share the same cellulose encapsulating properties as TBA, and may be a crucial ion in the cellulose dissolution process.

IL-co-solvent mixtures are capable of increasing cellulose dilution, creating a co-solvent concentration that maximizes the dissolution power of the solvent. This phenomenon is present in cellulose dissolution using 1-allyl-3-methylimidazolium chloride (AMIMCl) in DMSO, from 0 to $50 \mathrm{~mol} \%$ DMSO. ${ }^{102}$ In pure ILs, the cation and anion are typically located close to each other due to attractive Coulombic forces. ${ }^{58,62,102}$ The co-solvent can surround both the cation and anion, further separating the cation and anion, which allows them to act individually and in sequence during the cellulose separation process. ${ }^{58,62,102}$ Additionally, the co-solvent reduces viscosity and increases the diffusion of all the molecules in the solvent . 1,58,62,102 The decline of cellulose solubility at higher co-solvent concentrations is attributed to the loss in hydrogen bonding between IL and the cellulose bundle (i.e., mostly the anion) 
${ }^{102}$ In the TBPH-water solution, the co-solvent yields a maximum cellulose dissolution at approximately $91.1 \mathrm{~mol} \%$ water (see Figure 1.1). ${ }^{16}$ Since the two examples above are vastly different ILs and co-solvent mixtures, the cellulose dissolution maxima at the opposite ends of the co-solvent concentration is not an unexpected result. ${ }^{16,102}$

There are seemingly different cellulose solubility profiles when comparing the TBPHwater solution vs. the TBPCl-DMF or TBPCl-water solutions. ${ }^{8,16}$ Both the TBPH-water solution and TBPCl-water solution have similar structural, physical, and diffusive regime changes at $300 \mathrm{~K}$. Cellulose solubility in the TBPH-water solution maximizes between 86.8 to 93.9 mol \% water, in a Gaussian or maybe left-skewed Gaussian profile with a maximum at $91.1 \mathrm{~mol} \%$ water. The TBPCl-DMF and TBPCl-water data appear to show a continually decreasing cellulose solubility with increased co-solvent concentrations, with a more drastic decline after approximately $80 \mathrm{~mol} \%$ water. It is important to note that the TBPCl-water simulations were very far from equilibrium. Hence, a Gaussian profile or a significantly different profile is entirely possible under real experimental conditions where the dissolution times would be minutes to hours. The temperature of the solutions is one major difference for the dissolution profiles, with the TBPH-water solution being experimentally determined at $298 \mathrm{~K}$ temperature, the TBPCl-DMF solution experiment performed at $343 \mathrm{~K},{ }^{8}$ and the TBPCl-water simulations conducted at $360 \mathrm{~K}$.

Furthermore, despite the correlation between the experimental observed maximum dissolution of the TBPH-water solution, ${ }^{16}$ the water vein formation, and the diffusion regime shift observed in our MD simulations, there may be other mechanistic aspects at work that cannot be captured by the use of the classical, i.e., non-reactive, AMBER forcefield. ${ }^{103}$ Specifically, at low concentrations of water, the TBP and the hydroxide are capable of reacting. Other reactions may also occur such as the formation a $\mathrm{P}\left(\mathrm{C}_{4} \mathrm{H}_{9}\right)_{3}$ and $\mathrm{C}_{4} \mathrm{H}_{9} \mathrm{OH}$ or any other combination of the other butyl arm carbons. ${ }^{21,104}$ The formation of ylides and water may react in different ways with other molecules in the solution. ${ }^{21,104,105}$ Another possible reaction could occur between the phosphorous and hydroxide forming $\left(\mathrm{C}_{4} \mathrm{H}_{9}\right)_{3} \mathrm{POH}$. ${ }^{106}$ The increased length of the alkyl chains in the TBP molecule may make it less reactive than a tetramethylphosphonium or tetraethylphosphonium hydroxide, due to the steric hinderance in contacting the acidic hydrogen. ${ }^{105}$ Additionally, the TBPCl-water solution should 
be more stable than the TBPH-water solution, as the chloride anion is less reactive than the hydroxide anion. ${ }^{8,105}$ It is also possible that the mentioned reactions could contribute to the loss of cellulose dissolution in the TBPH-water solution at lower water concentrations, as the TBPCl-dimethylformamide (DMF) co-solvent mixture is capable of dissolving cellulose with the pure TBPCl IL at $343 \mathrm{~K}^{8}$ Additional experimental and theoretical work is required to determine all the reactions that are possible under the conditions and water concentrations in this document.

The most likely explanation for the differing TBPH-water and TBPCl-water solubility profiles is the lower temperatures and the reactivity of the TBPH-water solution at lower water concentrations. ${ }^{21,49,104,105}$ However, it is still possible that there are other mechanisms at play that are not captured in these studies or the present literature. More simulation and experimental work are required to confirm these assumptions and to design optimal IL co-solvent combinations for cellulose dissolution. If the solubility differences are due to temperature and the reactivity of the TBPH-water solution, many non-reactive TBP-based co-solvent solutions may also be capable of high cellulose dissolution at low to moderate temperatures. These TBP-based solution may also tolerate higher water concentrations, which would also make them economically attractive as cellulose solvents.

In the TBPCl-water simulations, the chloride-peeling strand net negative and the bundlepeeling strand net positive energy interaction is similar to the I- $\beta$ cellulose bundle dissolution in pure EMIM-Ac IL (alkylimidazolium-based IL), ${ }^{15}$ although the TBP-peeling strands have a favorable and increasingly negative pairwise energy, opposed to the largely unfavorable EMIM-peeling strand pairwise energy. ${ }^{15}$ The slightly net negative and favorable contribution of the TBP-peeling strand pairwise energy could assist the TBPCl-water mixture or the TBP family of ILs in dissolving cellulose at higher water concentrations for a given temperature.

At $300 \mathrm{~K}$, both the TBPCl-water and TBPH-water solutions show dramatic structural, physical, and diffusive regime changes from 80 to $92.5 \mathrm{~mol} \%$ water. These changes appear to stem from the interlocking structure of the TBP cations breaking down as the water forms veins throughout the system. In this region, the diffusion changes from a subdiffusive to a near-normal diffusive regime, allowing at least an order of magnitude increase in diffusion. The increased diffusion greatens the probability of interaction between the solvent and the 
cellulose strands, which is likely a crucial factor in cellulose dissolution. On the other hand, when the TBPCl-water solution is too diluted with water at around $93.9 \mathrm{~mol} \%$ water, there is insufficient interaction between the cation and anion with the cellulose. At $93.9 \mathrm{~mol} \%$ water, the $\mathrm{Cl}$ becomes mostly solvated with water, essentially destroying the hydrogen bonding lifetimes with cellulose atoms that are primarily responsible for the cellulose's intra-strand hydrogen bonds, thus eliminating cellulose dissolution. Cellulose dissolution begins to fail between 79.4 to 86.8 mol \% water, and the $\mathrm{Cl} \cdots \mathrm{HO} 2-\mathrm{O} 2$ and $\mathrm{Cl} \cdots \mathrm{HO} 3-\mathrm{O} 3$ hydrogen bond lifetimes go below the greatest of the intra-strand hydrogen bonding lifetimes (O5 ․ HO3$\mathrm{O} 3$ and $\mathrm{O} 6 \cdot \cdots \mathrm{HO} 2-\mathrm{O} 2)$. While this may not be the critical point of failure in all IL cosolvents, it could indicate the maximum co-solvent concentration failure point for similar dissolution mechanisms. Between 79.4 to 91.1 mol \% water, the increased diffusion likely helps to supplement the weakening of the chloride-intra-strand disrupting hydrogen bonding lifetimes via the water's increased assistance in intra-strand hydrogen disruption, preventing strand reformation, and allowing a lag time for the TBP molecule to move in and more permanently separate the cellulose strand. 


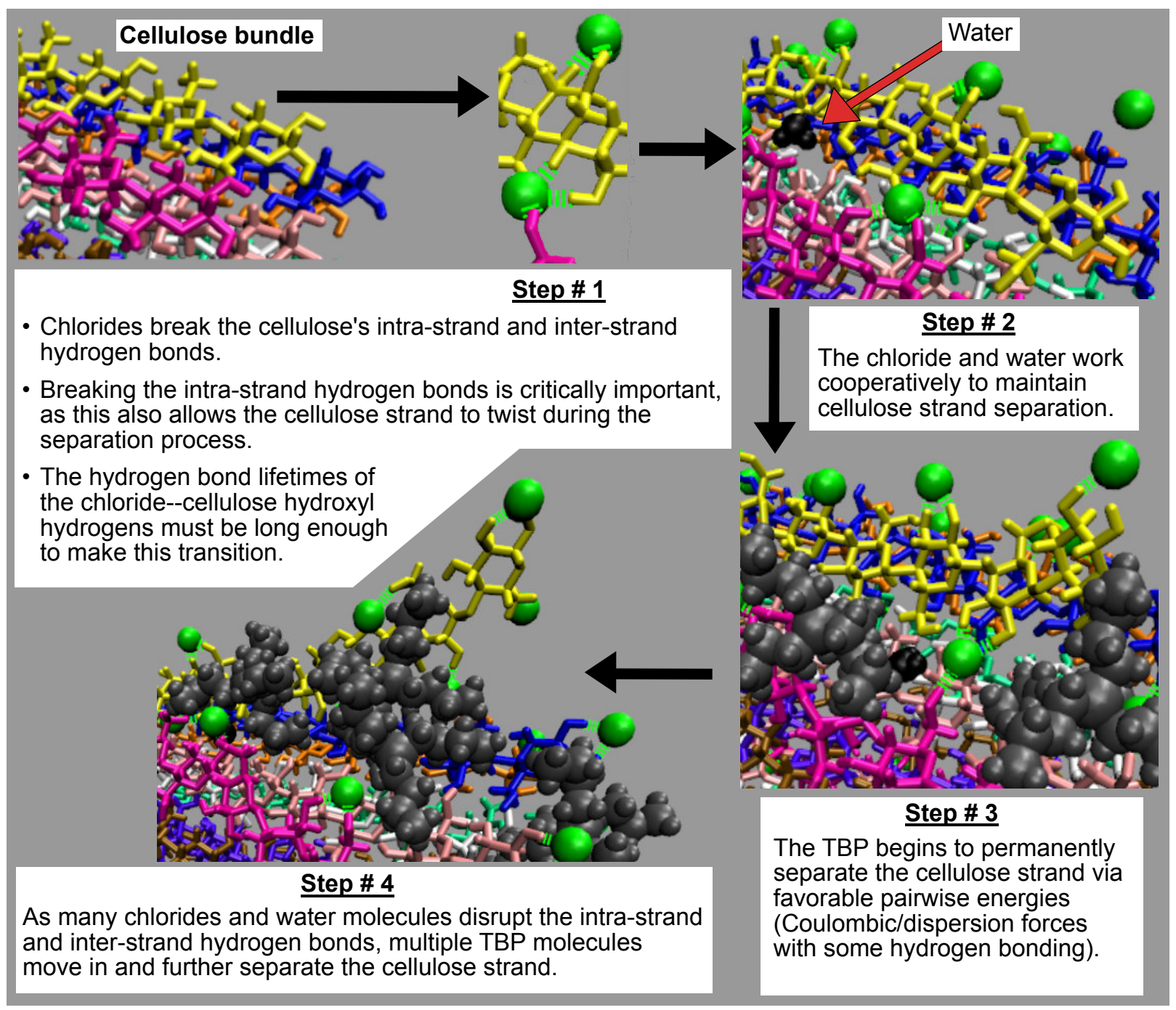

Figure (4.1) The summarized mechanism for cellulose dissolution in the TBPCl-water solution. These simulation snapshots are from the 63.1 mol \% water simulation. The green dashed lines indicate the chloride-cellulose hydrogen bonds. The water hydrogen bonds are not shown in this Figure. ${ }^{22}$

The cellulose dissolution mechanism for the TBPCl-water solution is broken down into four key steps and summarized in Figure 4.1. In step one, the chlorides must break the

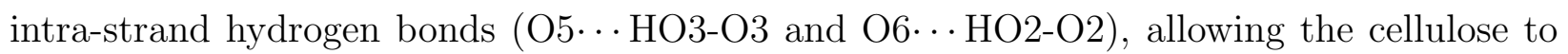
twist freely at the glycan connection point (i.e., 1,4-location). ${ }^{15} \mathrm{~A}$ single chloride is hydrogenbonded to both intra-strand hydrogen bonding locations, $\mathrm{Cl} \cdots \mathrm{O} 2-\mathrm{HO} 2$ and $\mathrm{Cl}$.. O3-HO3, at the same time (see the step \#1 image: the bottom hydrogen bonding in the yellow strand). The chlorides are very capable of breaking the intra-strand hydrogen bonds until the water 
concentration gets too high, around $80 \mathrm{~mol} \%$ water. Beyond $80 \mathrm{~mol} \%$ water, the $\mathrm{Cl} \cdots \mathrm{HO} 2$ $\mathrm{O} 2$ and $\mathrm{Cl} \cdots \mathrm{HO} 3-\mathrm{O} 3$ hydrogen bonding lifetimes fall below the intra-strand hydrogen lifetimes, reducing the probability of sustained intra-strand hydrogen bonding disruption (see Figure 3.26). The increased diffusion of the TBPCl-water solution above $80 \mathrm{~mol} \%$ water may slow the rapid decline of the cellulose dissolution (see Figure 3.17). ${ }^{58}$ The chlorides can also disrupt the inter-strand hydrogen bonds, disconnecting the strand from its neighboring strand. The chloride anions have a net negative pairwise interaction with the cellulose strand, while being able to hold multiple hydrogen bonds with the cellulose strand/bundle. The net negative pairwise interaction of the chloride offsets the net positive pairwise interaction of the peeling cellulose strand, making the process more thermodynamically feasible. ${ }^{15}$ In step two, the chlorides and water form small gaps between the strand and its neighboring strands via physical separation and hydrogen bonding. These small gaps delay the reformation of the cellulose strand, so the TBP cation has time to finish the strand separation before the cellulose strand reforms. Water is capable of hydrogen bonding to the cellulose strand for shorter durations. The water hydrogen bonds are not shown in Figure 4.1, but are shown in Figure 3.32. In step three, the TBP moves in and further and more permanently separates the cellulose strand. The Coulombic and dispersion forces, hydrogen bonding, and the shape of the TBP molecule allows it to separate the cellulose strand effectively. Additionally, the TBP cations, at worst, have an overall net neutral pairwise interaction with the cellulose strand, and a favorable pairwise interaction with the peeling cellulose strand. A unique attribute of the TBP cation when compared to the imidazolium cation, is the consistently favorable (negative) pairwise energy throughout the entire dissolution process. ${ }^{15}$ In step four, more TBP molecules move in and separate the strand further. At the same time, the chloride hydrogen bonding is still providing flexibility to the separating strand, while again using the attributes of steps one, two, and three.

The goal of the present research is to understand the structural, physical, and mechanical properties of the TBPCl-water mixture, or the TBP-anion-water family of ILs, that make them so effective in dissolving cellulose. There is sufficient theoretical and experimental evidence indicating that the specific anion, cation, and co-solvent each play a part in the solution's ability to dissolve. ${ }^{8,15,16,19,62,78,90,101,102,107}$ The solution's structural properties, hy- 
drogen bonding, pairwise energy interactions, and diffusion all appear to play a role in the dissolution power of the IL and co-solvent. ${ }^{15,22,58,62,93,102}$ This implies that the properties of each cation and co-solvent class of ILs will require individual study, and some of the critical properties may not be assumed when switching to a different solvent class. These TBPbased solution may also tolerate higher water concentrations, which would also make them economically attractive as cellulose solvents. 


\section{Conclusions}

The TBPCl-water simulations illustrate the potential for dissolving cellulose and identify some properties and mechanisms which drive the process. The individual solvent properties align with some unique regions where the cellulose solubility is undergoing significant changes. The principle results and novelty of this research are presented below:

1. The properties of the TBPCl-water and TBPH-water solutions which were previously unavailable, were calculated.

(a) The physical properties that were the focus of the simulations were the density, excess molar volume, hydrogen bonding, and radial distribution functions.

(b) The calculated thermodynamic properties include the excess enthalpy of mixing, heat capacity $\left(c_{p}\right)$, and thermal expansivity $\left(\alpha_{\mathrm{p}}\right)$.

(c) The transport properties were analyzed via the anomalous diffusion coefficients and the ergodicity breaking parameter.

2. The TBPCl-water and TBPH-water solutions exhibit unique water vein formation between 80 to $92.5 \mathrm{~mol} \%$ water.

(a) The water veins form throughout the solution breaking the TBPs interlocking arms and allowing molecules to diffuse freely throughout the solution.

(b) The diffusion of the system increases at least an order of magnitude, shifting from a subdiffusive to a near-normal diffusive regime.

(c) Below $80 \mathrm{~mol} \%$ water, the trapping and caging structure of the TBP arms contain the water molecules to specific water pocket, leading to very low diffusion at these lower water concentrations. 
3. The intra-strand (within a strand), inter-strand (between strands), and TBPCl-water (solvent)-strand hydrogen bonding was compared at various IL-water concentrations between a small and large $I \beta$ cellulose bundle (18 vs. 88 strands).

(a) The intra-strand hydrogen bonding for the solvent-exposed strands was nearly the same between the small and large $I \beta$ cellulose bundles. The center strands in the small bundle had significantly less intra-strand hydrogen bonds, due to less bundle stabilization from the other cellulose strands when compared to the large cellulose bundle. This is an important result, as the solvent-exposed strands of the small and large bundles must have matching intra-strand hydrogen bonds, since breaking them is the critical first step in the IL-based cellulose dissolution process.

(b) The inter-strand bonding was significantly different between the small and large bundles, except for the $63.1 \mathrm{~mol} \%$ water concentration, where the large bundle was nearly identical to the small bundle's inter-strand bonding. However, more research is required to determine precisely why the inter-strand hydrogen bonding is the same at $63.1 \mathrm{~mol} \%$ water but differs for all the higher concentrations. The nearly identical inter-strand hydrogen bonding at $63.1 \mathrm{~mol} \%$ water could be an artifact of the GLYCAM06 ${ }^{24}$ force field with the low dispersion and Coulombic forces near the cellulose at the start of the simulations (i.e., the void space of at least $2 \AA$ from the cellulose bundles at the start of the simulation).

(c) The TBPCl-water (solvent)-strand hydrogen bonding was nearly the same between the small and large $I \beta$ cellulose bundles.

(d) Overall, the small bundle's solvent-exposed strands appear to be a fair representation of the large cellulose bundle in the TBPCl-water system. For many similar cases, the small bundle may be an adequate replacement for the larger cellulose bundle, while significantly minimizing the computational expense of the simulations. 


\section{Cellulose dissolution in the TBPCl-water solution}

(a) These simulations determined that cellulose dissolution is possible using high water concentrations at moderate temperatures. An estimated cellulose solubility profile was generated for the TBPCl-water solution.

(b) The TBP-peeling strands have a favorable (negative) pairwise energy throughout the entire dissolution process, as opposed to the at-times largely unfavorable (positive) EMIM-peeling strand pairwise energy. ${ }^{15}$ The consistently favorable (negative) pairwise energy between the TBP-peeling strands is partially attributed to its success in dissolving cellulose.

(c) A quantifiable attribute of the cellulose dissolution process was determined by calculating the anion...HO2-O2 and anion...HO3-O3 hydrogen bonding lifetimes (i.e., anion disrupting the cellulose's intra-strand hydrogen bonding). These lifetimes may identify the theoretical maximum co-solvent concentration before the cellulose dissolution rapidly declines. Once the anion $\cdots$ HO2-O2 and anion $\cdots$ HO3O3 hydrogen bonding lifetimes fall below the largest intra-strand hydrogen bonding lifetime, the solution's cellulose solubility rapidly decays.

(d) In the TBPCl-water solution between 79.4 to 91.1 mol \% water, the dramatically increased diffusion combined with the localized hydrogen bonding of water assists in the cellulose dissolution, despite declining $\mathrm{Cl} \cdots \mathrm{HO} 2-\mathrm{O} 2$ and $\mathrm{Cl} \cdots \mathrm{HO} 3-\mathrm{O} 3$ hydrogen bonding lifetimes. Water physically prevents the reformation of the cellulose strand and provides a lag time for the TBP molecule to move in and more permanently separate the cellulose strand. While this is not an all-inclusive list, the following hydrogen bonds strongly assist in attracting and holding water between the separating cellulose strands:

- The moderate strength of the Ow $\cdots$ HO2-O2 and Ow..HO6-O6 hydrogen bonds (i.e., water-cellulose hydrogen bonds).

- The moderate strength of the $\mathrm{Cl} \cdots \mathrm{Hw}-\mathrm{Ow}$ hydrogen bond (i.e., Cl-water hydrogen bonds) when the $\mathrm{Cl}$ is near or hydrogen-bonded to the cellulose strand. 
(e) A cooperative mechanism was defined, where the water, $\mathrm{Cl}$, and TBP molecules all work together to enable the cellulose dissolution process until the solution becomes too diluted with water ( $\geq 93.9 \mathrm{~mol} \%$ water $)$. 


\section{Future Work}

The use of machine learning algorithms may accelerate the discovery of new IL co-solvent combinations for cellulose dissolution and could identify more economical and environmentallyfriendly solutions. However, these machine learning algorithms require some design and selection parameters to be effective when selecting potential IL co-solvent solutions. Combining this research and the work of others could provide the initial round of design parameters for the machine learning algorithm. The results of this research could be optimized to be more computationally efficient, allowing these algorithms to be conducted with a reasonable computational cost. Any final solvents selected via the machine learning algorithm must be experimentally validated, as with any calculated or theorized conclusion.

One of the major findings of this research was the quantifying the rapid decay of the hydrogen bonding lifetimes for the chloride-cellulose hydroxyl hydrogens with increasing water concentration. Once the chloride-cellulose hydroxyl hydrogens lifetimes fall below the largest cellulose intra-strand hydrogen bonds (i.e., the potential hydrogen bonding lifetime threshold for the chloride-cellulose hydroxyl hydrogens), cellulose dissolution rapidly decays. The cation and co-solvent likely influence these critical lifetimes, so every combination should be evaluated. However, at the current scale of these simulations, it is not computationally feasible to use even the 18 strand cellulose bundle with a machine learning algorithm, as it would still require at least $200 \mathrm{~ns}$ of simulation time for the 100,000 atom system. This system could be systematically scaled down to several cellulose strands with a few glycans per strand (say 8-10 strands with 6-8 glycans), while maintaining reasonable accuracy with regard to the intra-strand, inter-strand, and solvent-strand hydrogen bonding. This could be accomplished with simulations that incorporate the ends of the cellulose bundle, like the simulations in this study, or making them continuous strands through the periodic boundary condition (i.e., a cellulose bundle/strand without ends or an infinitely long bundle/strand). If this can be accomplished with reasonable accuracy, the computational cost of the simulations could be reduced by approximately 4 to 20 times, when compared with the cellulose simulations in this study. Additionally, combining the cellulose strand/glycan reduction, the OPLS-AA force field, ${ }^{35,108,109}$ and other speedup methods such as the Isele-Holder method with RESPA 
could reduce the total computational cost by approximately 8 to 40 times when compared with the cellulose simulations in this study. ${ }^{37,51}$

Others have discovered that the anion's shape, size, the hydrogen bonding orientation with the cellulose, and the ability to have multiple hydrogen bonds with the cellulose are critical factors in cellulose dissolution since they are responsible for the intra-strand and inter-strand hydrogen bonding disruption. 8,15,19,78,90,93,101,102,102,107 The intra-stand and interstrand hydrogen bonding of the cellulose is also critical to the cellulose dissolution and can be analyzed with future machine learning algorithms. The cellulose dissolution also depends on how the polar and non-polar parts of the cellulose strand interact with the solvent, in many cases occurring between the cation and the peeling strand. ${ }^{1,15,110}$ The pairwise energy between the peeling cellulose strand and the cation also seems to be a factor in the cellulose dissolution. ${ }^{15}$ If all the factors mentioned in this section, and others deemed relevant (i.e., solvent diffusion, viscosity, temperature, cellulose strand twisting, etc.), 1,25,26,62,82,102 are trained into a machine learning algorithm, which requires 10,000 atoms or less, it should be computationally feasible. Future researchers should focus on scaling down the cellulose bundle simulations with the IL and co-solvent, incorporating the critical parameters into a machine learning algorithm, and utilize the OPLS-AA force field with the Isele-Holder method with RESPA. ${ }^{35,37,51,108,109}$ The Molecular Simulation Design Framework (MoSDeF) would be an ideal tool for creating and modifying the systems based on the feedback from a machine learning algorithm. ${ }^{82,111-115}$ 


\section{Appendix}

The force field parameters for the molecular dynamics simulations are provided. Additional visualizations of the molecular dynamics simulations include the water vein and channeling pictures for the TBPH-water solutions, the RDFs for the TBPH-water solutions, and additional diffusion data for the TBPH-water system. The TBPH water vein/channeling formation from the molecular dynamics simulations are included to provide a clearer understanding of the structural changes that occur throughout the solution with increasing water concentrations. These water vein pictures used the TIP3P-pppm water model, which differs

from the rest of the TIP4P/2005 water model simulations. ${ }^{49,53,54}$ The TBPH-water RDFs are included as well, which were not provided in the main body of this document. The diffusion data consist of the ergodicity breaking parameters, additional particle-averaged TAMSDs, and MSDs for the TBPH-water system.

Additional data are provided for the small $I \beta$ cellulose bundle dissolution in the TBPClwater simulations to paint a clear picture of the whole dissolution process. The cellulose dissolution is provided in the number of dissolved glycans. The additional cellulose dissolution images, pairwise energies and hydrogen bonding of the individual separating yellow strands or non-separating yellow strands, are provided below. Several other hydrogen bonding lifetimes were also calculated in this study and are included, primarily including the lifetimes between the cellulose bundle itself. The diffusion of singular water molecules and the number of molecules between the peeling strand and an inner strand of the cellulose bundle are also provided. 
7.1 Methodology (cont.) 
Table (7.1) Atomic charges

\begin{tabular}{|c|c|}
\hline Atom Type & $q(e)$ \\
\hline $\mathrm{C} 1^{24}$ & 0.378 \\
\hline $\mathrm{C} 2^{24}$ & 0.31 \\
\hline $\mathrm{C} 3^{24}$ & 0.284 \\
\hline $\mathrm{C} 4^{24}$ & 0.267 \\
\hline $\mathrm{C} 5^{24}$ & 0.225 \\
\hline $\mathrm{C} 6^{24}$ & 0.282 \\
\hline $\mathrm{CT} 1^{36}$ & -0.0070 \\
\hline $\mathrm{CT} 2^{36}$ & -0.0155 \\
\hline $\mathrm{CT}^{36}$ & 0.0089 \\
\hline $\mathrm{CT} 4^{36}$ & -0.0776 \\
\hline $\mathrm{Cl}^{34,35}$ & -1.0 \\
\hline $\mathrm{Oh}^{48}$ & -1.35 \\
\hline $\mathrm{Hh}^{48}$ & 0.35 \\
\hline $\mathrm{H} 1^{24}$ & 0 \\
\hline $\mathrm{H} 2^{24}$ & 0 \\
\hline $\mathrm{H} 3^{24}$ & 0 \\
\hline $\mathrm{H} 4^{24}$ & 0 \\
\hline $\mathrm{H} 5^{24}$ & 0 \\
\hline $\mathrm{H} 61^{24}$ & 0 \\
\hline $\mathrm{H} 62^{24}$ & 0 \\
\hline $\mathrm{HC} 2^{36}$ & 0.0204 \\
\hline HC $3^{36}$ & 0.0228 \\
\hline $\mathrm{HC} 4^{36}$ & 0.0342 \\
\hline $\mathrm{HO}^{24}$ & 0.44 \\
\hline $\mathrm{HO} 2^{24}$ & 0.437 \\
\hline $\mathrm{HO}^{24}$ & 0.432 \\
\hline $\mathrm{HO} 4^{24}$ & 0.44 \\
\hline $\mathrm{HO} 6^{24}$ & 0.424 \\
\hline $\mathrm{HP}^{36}$ & 0.0500 \\
\hline Hw (TIP3P-pppm) $)^{53,54}$ & 0.415 \\
\hline $\mathrm{Hw}(\mathrm{TIP} 4 \mathrm{P} / 2005)^{49}$ & 0.5564 \\
\hline $\mathrm{O} 1^{24}$ & -0.62 \\
\hline $\mathrm{O} 2^{24}$ & -0.718 \\
\hline $\mathrm{O} 3^{24}$ & -0.709 \\
\hline $\mathrm{O} 4^{24}$ & -0.454 \\
\hline O4B (terminating end) & -0.714 \\
\hline $\mathrm{O} 5^{24}$ & -0.47 \\
\hline O $6^{24}$ & -0.688 \\
\hline Ow (TIP3P-pppm) $)^{53,54}$ & -0.830 \\
\hline Ow $(\mathrm{TIP} 4 \mathrm{P} / 2005)^{49}$ & -1.1128 \\
\hline $\mathrm{P}^{36}$ & 0.2088 \\
\hline
\end{tabular}


Table (7.2) Pair coefficients

\begin{tabular}{|c|c|c|}
\hline Atom Type-Atom Type & $\begin{array}{c}\epsilon \\
\left(\text { kcal } m o l^{-1}\right)\end{array}$ & $\begin{array}{c}\sigma \\
(\AA)\end{array}$ \\
\hline $\mathrm{C} 1-\mathrm{C} 1^{24}$ & 0.109400 & 3.3997 \\
\hline $\mathrm{C} 2-\mathrm{C} 2^{24}$ & 0.109400 & 3.3997 \\
\hline $\mathrm{C} 3-\mathrm{C} 3^{24}$ & 0.109400 & 3.3997 \\
\hline $\mathrm{C} 4-\mathrm{C} 4^{24}$ & 0.109400 & 3.3997 \\
\hline $\mathrm{C} 5-\mathrm{C} 5^{24}$ & 0.109400 & 3.3997 \\
\hline $\mathrm{C} 6-\mathrm{C} 6^{24}$ & 0.109400 & 3.3997 \\
\hline $\mathrm{CT} 1-\mathrm{CT} 1^{36}$ & 0.109320 & 3.3997 \\
\hline $\mathrm{CT} 2-\mathrm{CT} 2^{36}$ & 0.109320 & 3.3997 \\
\hline $\mathrm{CT} 3-\mathrm{CT} 3^{36}$ & 0.109320 & 3.3997 \\
\hline $\mathrm{CT} 4-\mathrm{CT} 4^{36}$ & 0.109320 & 3.3997 \\
\hline $\mathrm{Cl}-\mathrm{Cl}^{34,35}$ & 0.148 & 3.77 \\
\hline Oh-Oh (hydroxide oxygen) ${ }^{48}$ & 0.155896 & 3.7300 \\
\hline Hh-Hh (hydroxide hydrogen) ${ }^{48}$ & 0.000000 & 0.0000 \\
\hline $\mathrm{H} 1-\mathrm{H} 1^{24}$ & 0.015700 & 2.2932 \\
\hline $\mathrm{H} 2-\mathrm{H} 2^{24}$ & 0.015700 & 2.4714 \\
\hline $\mathrm{H} 3-\mathrm{H} 3^{24}$ & 0.015700 & 2.4714 \\
\hline $\mathrm{H} 4-\mathrm{H} 4^{24}$ & 0.015700 & 2.4714 \\
\hline $\mathrm{H} 5-\mathrm{H} 5^{24}$ & 0.015700 & 2.4714 \\
\hline $\mathrm{H} 61-\mathrm{H} 61^{24}$ & 0.015700 & 2.4714 \\
\hline $\mathrm{H} 62-\mathrm{H} 62^{24}$ & 0.015700 & 2.4714 \\
\hline $\mathrm{HC} 2-\mathrm{HC} 2^{36}$ & 0.015692 & 2.6495 \\
\hline $\mathrm{HC} 3-\mathrm{HC} 3^{36}$ & 0.015692 & 2.6495 \\
\hline $\mathrm{HC} 4-\mathrm{HC} 4^{36}$ & 0.015692 & 2.6495 \\
\hline $\mathrm{HO} 1-\mathrm{HO}^{24}$ & 0.030000 & 0.3564 \\
\hline $\mathrm{HO} 2-\mathrm{HO} 2^{24}$ & 0.030000 & 0.3564 \\
\hline $\mathrm{HO} 3-\mathrm{HO}^{24}$ & 0.030000 & 0.3564 \\
\hline $\mathrm{HO} 4-\mathrm{HO} 4^{24}$ & 0.030000 & 0.3564 \\
\hline $\mathrm{HO} 6-\mathrm{HO} 6^{24}$ & 0.030000 & 0.3564 \\
\hline $\mathrm{HP}-\mathrm{HP}^{36}$ & 0.015692 & 1.9600 \\
\hline Hw (TIP3P-pppm)-Hw (TIP3P-pppm) $)^{53,54}$ & 0.000000 & 0.0000 \\
\hline Hw (TIP4P/2005)-Hw (TIP4P/2005) 49 & 0.000000 & 0.0000 \\
\hline $\mathrm{O} 1-\mathrm{O} 1^{24}$ & 0.210400 & 3.0665 \\
\hline $\mathrm{O} 2-\mathrm{O} 2^{24}$ & 0.210400 & 3.0665 \\
\hline $\mathrm{O} 3-\mathrm{O} 3^{24}$ & 0.210400 & 3.0665 \\
\hline $\mathrm{O} 4-\mathrm{O} 4^{24}$ & 0.170000 & 3.0000 \\
\hline $\mathrm{O} 4 \mathrm{~B}-\mathrm{O} 4 \mathrm{~B}$ (terminating end) ${ }^{24}$ & 0.210400 & 3.0665 \\
\hline $\mathrm{O} 5-\mathrm{O} 5^{24}$ & 0.170000 & 3.0000 \\
\hline $\mathrm{O} 6-\mathrm{O} 6^{24}$ & 0.210400 & 3.0665 \\
\hline Ow (TIP3P-pppm)-Ow (TIP3P-pppm) $)^{53,54}$ & 0.102000 & 3.1880 \\
\hline Ow (TIP4P/2005)-Ow (TTIP4P/2005) 49 & 0.1852 & 3.1589 \\
\hline $\mathrm{P}-\mathrm{P}^{36}$ & 0.199866 & 3.7418 \\
\hline
\end{tabular}

The Lorentz-Berthelot ${ }^{32,33}$ mixing rules were utilized. 102 
Table (7.3) Bond coefficients (Part 1 of 2)

\begin{tabular}{ccc}
\hline \hline & $K_{r}$ & $r_{0}$ \\
Atom Type-Atom Type & $\left(\mathrm{kcal} \mathrm{mol}^{-1} \AA^{-2}\right)$ & $(\AA)$ \\
\hline $\mathrm{C} 1-\mathrm{C} 2^{24}$ & 310 & 1.52 \\
$\mathrm{C} 1-\mathrm{H} 1^{24}$ & 340 & 1.09 \\
$\mathrm{C} 1-\mathrm{O} 1^{24}$ & 320 & 1.43 \\
$\mathrm{C} 1-\mathrm{O} 4^{24}$ & 285 & 1.46 \\
$\mathrm{C} 1-\mathrm{O} 5^{24}$ & 285 & 1.46 \\
$\mathrm{C} 2-\mathrm{C} 3^{24}$ & 310 & 1.52 \\
$\mathrm{C} 2-\mathrm{H} 2^{24}$ & 340 & 1.09 \\
$\mathrm{C} 2-\mathrm{O} 2^{24}$ & 320 & 1.43 \\
$\mathrm{C} 3-\mathrm{C} 4^{24}$ & 310 & 1.52 \\
$\mathrm{C} 3-\mathrm{H} 3^{24}$ & 340 & 1.09 \\
$\mathrm{C} 3-\mathrm{O} 3^{24}$ & 320 & 1.43 \\
$\mathrm{C} 4-\mathrm{C} 5^{24}$ & 310 & 1.52 \\
$\mathrm{C} 4-\mathrm{H} 4^{24}$ & 340 & 1.09 \\
$\mathrm{C} 4-\mathrm{O} 4^{24}$ & 285 & 1.46 \\
$\mathrm{C} 4-\mathrm{O} 4 \mathrm{~B}^{24}$ & 320 & 1.43 \\
$\mathrm{C} 5-\mathrm{C} 6^{24}$ & 310 & 1.52 \\
$\mathrm{C} 5-\mathrm{H} 5^{24}$ & 340 & 1.09 \\
$\mathrm{C} 5-\mathrm{O} 5^{24}$ & 285 & 1.46 \\
$\mathrm{C} 6-\mathrm{H} 61^{24}$ & 340 & 1.09 \\
$\mathrm{C} 6-\mathrm{H} 62^{24}$ & 340 & 1.09 \\
$\mathrm{C} 6-O 6^{24}$ & 320 & 1.43 \\
$\mathrm{HO} 1-O 1^{24}$ & 553 & 0.96 \\
$\mathrm{HO} 2-\mathrm{O} 2^{24}$ & 553 & 0.96 \\
$\mathrm{HO} 3-3^{24}$ & 553 & 0.96 \\
$\mathrm{HO} 4-\mathrm{O} 4 \mathrm{~B}^{24}$ & 553 & 0.96 \\
$\mathrm{HO} 6-O 6^{24}$ & 553 & 0.96 \\
\hline \hline
\end{tabular}


Table (7.4) Bond coefficients (Part 2 of 2)

\begin{tabular}{|c|c|c|}
\hline Bond Type & $\begin{array}{c}K_{r} \\
\left(\mathrm{kcal} m o l^{-1} \AA^{-2}\right)\end{array}$ & $\begin{array}{c}r_{0} \\
(\AA)\end{array}$ \\
\hline CT1-CT2 ${ }^{36}$ & 309.793 & 1.5334 \\
\hline $\mathrm{CT} 1-\mathrm{HP}^{36}$ & 336.276 & 1.0862 \\
\hline CT1-P 36 & 209.86 & 1.8273 \\
\hline $\mathrm{CT} 2-\mathrm{CT} 3^{36}$ & 309.793 & 1.5334 \\
\hline $\mathrm{CT} 2-\mathrm{HC} 2^{36}$ & 330.778 & 1.0860 \\
\hline CT3-CT4 ${ }^{36}$ & 309.793 & 1.5334 \\
\hline $\mathrm{CT} 3-\mathrm{HC} 3^{36}$ & 330.778 & 1.0860 \\
\hline $\mathrm{CT} 4-\mathrm{HC} 4^{36}$ & 330.778 & 1.0860 \\
\hline Hh-Oh (hydroxide) ${ }^{48}$ & 500 & 1.0000 \\
\hline Hw-Ow (TIP3P-pppm) ${ }^{53,54}$ & 450 & 0.9572 \\
\hline $\mathrm{Hw}-\mathrm{Ow}(\mathrm{TIP} 4 \mathrm{P} / 2005)^{49}$ & 500 & 0.9572 \\
\hline
\end{tabular}


Table (7.5) Angle coefficients (Part 1 of 2)

\begin{tabular}{|c|c|c|}
\hline Angle Type & $\begin{array}{c}K_{\theta} \\
\left(\text { kcal } \mathrm{mol}^{-1} \operatorname{rad}^{-2}\right)\end{array}$ & $\begin{array}{c}\theta_{0} \\
(\mathrm{deg})\end{array}$ \\
\hline $\mathrm{C} 1-\mathrm{C} 2-\mathrm{C} 3^{24}$ & 45 & 113.5 \\
\hline $\mathrm{C} 1-\mathrm{C} 2-\mathrm{H} 2^{24}$ & 45 & 111.0 \\
\hline $\mathrm{C} 1-\mathrm{C} 2-\mathrm{O} 2^{24}$ & 70 & 107.5 \\
\hline $\mathrm{C} 1-\mathrm{O} 1-\mathrm{HO}^{24}$ & 55 & 109.5 \\
\hline $\mathrm{C} 1-\mathrm{O} 4-\mathrm{C} 4^{24}$ & 50 & 111.6 \\
\hline $\mathrm{C} 1-\mathrm{O} 5-\mathrm{C} 5^{24}$ & 50 & 111.6 \\
\hline $\mathrm{C} 2-\mathrm{C} 1-\mathrm{H} 1^{24}$ & 45 & 111.0 \\
\hline $\mathrm{C} 2-\mathrm{C} 1-\mathrm{O} 1^{24}$ & 70 & 107.5 \\
\hline $\mathrm{C} 2-\mathrm{C} 1-\mathrm{O} 4^{24}$ & 70 & 108.5 \\
\hline $\mathrm{C} 2-\mathrm{C} 1-\mathrm{O} 5^{24}$ & 70 & 108.5 \\
\hline $\mathrm{C} 2-\mathrm{C} 3-\mathrm{C} 4^{24}$ & 45 & 113.5 \\
\hline $\mathrm{C} 2-\mathrm{C} 3-\mathrm{H} 3^{24}$ & 45 & 111.0 \\
\hline $\mathrm{C} 2-\mathrm{C} 3-\mathrm{O} 3^{24}$ & 70 & 107.5 \\
\hline $\mathrm{C} 2-\mathrm{O} 2-\mathrm{HO} 2^{24}$ & 55 & 109.5 \\
\hline $\mathrm{C} 3-\mathrm{C} 2-\mathrm{H} 2^{24}$ & 45 & 111.0 \\
\hline $\mathrm{C} 3-\mathrm{C} 2-\mathrm{O} 2^{24}$ & 70 & 107.5 \\
\hline $\mathrm{C} 3-\mathrm{C} 4-\mathrm{C} 5^{24}$ & 45 & 113.5 \\
\hline $\mathrm{C} 3-\mathrm{C} 4-\mathrm{H} 4^{24}$ & 45 & 111.0 \\
\hline $\mathrm{C} 3-\mathrm{C} 4-\mathrm{O} 4^{24}$ & 70 & 108.5 \\
\hline $\mathrm{C} 3-\mathrm{C} 4-\mathrm{O} 4 \mathrm{~B}^{24}$ & 70 & 107.5 \\
\hline $\mathrm{C} 3-\mathrm{O} 3-\mathrm{HO}^{24}$ & 55 & 109.5 \\
\hline $\mathrm{C} 4-\mathrm{C} 3-\mathrm{H} 3^{24}$ & 45 & 111.0 \\
\hline $\mathrm{C} 4-\mathrm{C} 3-\mathrm{O} 3^{24}$ & 70 & 107.5 \\
\hline $\mathrm{C} 4-\mathrm{C} 5-\mathrm{C} 6^{24}$ & 45 & 113.5 \\
\hline $\mathrm{C} 4-\mathrm{C} 5-\mathrm{H} 5^{24}$ & 45 & 111.0 \\
\hline $\mathrm{C} 4-\mathrm{C} 5-\mathrm{O} 5^{24}$ & 70 & 108.5 \\
\hline $\mathrm{C} 4-\mathrm{O} 4 \mathrm{~B}-\mathrm{HO} 4^{24}$ & 55 & 109.5 \\
\hline $\mathrm{C} 5-\mathrm{C} 4-\mathrm{H} 4^{24}$ & 45 & 111.0 \\
\hline $\mathrm{C} 5-\mathrm{C} 4-\mathrm{O} 4^{24}$ & 70 & 108.5 \\
\hline $\mathrm{C} 5-\mathrm{C} 4-\mathrm{O} 4 \mathrm{~B}^{24}$ & 70 & 107.5 \\
\hline C5-C6-H61 ${ }^{24}$ & 45 & 111.0 \\
\hline $\mathrm{C} 5-\mathrm{C} 6-\mathrm{H} 62^{24}$ & 45 & 111.0 \\
\hline $\mathrm{C} 5-\mathrm{C} 6-\mathrm{O} 6^{24}$ & 70 & 107.5 \\
\hline C6-C5-H $5^{24}$ & 45 & 111.0 \\
\hline $\mathrm{C} 6-\mathrm{C} 5-\mathrm{O} 5^{24}$ & 70 & 108.5 \\
\hline $\mathrm{C} 6-\mathrm{O} 6-\mathrm{HO}^{24}$ & 55 & 109.5 \\
\hline
\end{tabular}


Table (7.6) Angle coefficients (Part 2 of 2)

\begin{tabular}{|c|c|c|}
\hline Angle Type & $\begin{array}{c}K_{\theta} \\
\left(\mathrm{kcal} \mathrm{mol}^{-1} \mathrm{rad}^{-2}\right)\end{array}$ & $\begin{array}{c}\theta_{0} \\
(\mathrm{deg})\end{array}$ \\
\hline CT1-CT2-CT3 ${ }^{36}$ & 40.9716 & 111.88 \\
\hline $\mathrm{CT} 1-\mathrm{CT} 2-\mathrm{HC} 2^{36}$ & 42.4716 & 109.96 \\
\hline $\mathrm{CT} 1-\mathrm{P}-\mathrm{CT} 1^{36}$ & 49.9666 & 109.46 \\
\hline $\mathrm{CT} 2-\mathrm{CT} 1-\mathrm{HP}^{36}$ & 30.9783 & 110.07 \\
\hline $\mathrm{CT} 2-\mathrm{CT} 1-\mathrm{P}^{36}$ & 74.9498 & 116.24 \\
\hline CT2-CT3-CT4 ${ }^{36}$ & 40.9716 & 111.88 \\
\hline $\mathrm{CT} 2-\mathrm{CT} 3-\mathrm{HC}^{36}$ & 42.4716 & 109.96 \\
\hline CT3-CT2-HC2 ${ }^{36}$ & 42.4716 & 109.96 \\
\hline CT3-CT4-HC4 ${ }^{36}$ & 42.4716 & 109.96 \\
\hline CT4-CT3-HC3 ${ }^{36}$ & 42.4716 & 109.96 \\
\hline H1-C1-O1 24 & 60.0 & 110.0 \\
\hline $\mathrm{H} 1-\mathrm{C} 1-\mathrm{O} 4{ }^{24}$ & 60.0 & 110.0 \\
\hline $\mathrm{H} 1-\mathrm{C} 1-\mathrm{O} 5^{24}$ & 60.0 & 110.0 \\
\hline $\mathrm{H} 2-\mathrm{C} 2-\mathrm{O} 2^{24}$ & 60.0 & 110.0 \\
\hline $\mathrm{H} 3-\mathrm{C} 3-\mathrm{O} 3{ }^{24}$ & 60.0 & 110.0 \\
\hline $\mathrm{H} 4-\mathrm{C} 4-\mathrm{O} 4^{24}$ & 60.0 & 110.0 \\
\hline $\mathrm{H} 4-\mathrm{C} 4-\mathrm{O} 4 \mathrm{~B}^{24}$ & 60.0 & 110.0 \\
\hline $\mathrm{H} 5-\mathrm{C} 5-\mathrm{O} 5^{24}$ & 60.0 & 110.0 \\
\hline $\mathrm{H} 61-\mathrm{C} 6-\mathrm{H} 62^{24}$ & 45.0 & 109.5 \\
\hline $\mathrm{H} 61-\mathrm{C} 6-\mathrm{O}^{24}$ & 60.0 & 110.0 \\
\hline $\mathrm{H} 62-\mathrm{C} 6-\mathrm{O} 6^{24}$ & 60.0 & 110.0 \\
\hline $\mathrm{HC} 2-\mathrm{CT} 2-\mathrm{HC} 2^{36}$ & 38.2751 & 106.47 \\
\hline HC3-CT3-HC3 ${ }^{36}$ & 38.2751 & 106.47 \\
\hline HC4-CT4-HC4 ${ }^{36}$ & 38.2751 & 106.47 \\
\hline HP-CT1-HP 36 & 36.6748 & 107.36 \\
\hline HP-CT1-P ${ }^{36}$ & 32.4783 & 106.74 \\
\hline Hw-Ow-Hw (TIP3P-pppm) $)^{53,54}$ & 55 & 104.52 \\
\hline Hw-Ow-Hw (TIP4P-2005) 49 & 100 & 104.52 \\
\hline $\mathrm{O} 1-\mathrm{C} 1-\mathrm{O} 5^{24}$ & 100 & 112.0 \\
\hline $\mathrm{O} 4-\mathrm{C} 1-\mathrm{O} 5^{24}$ & 100 & 112.0 \\
\hline
\end{tabular}


Table (7.7) Torsion/dihedral angle coefficients (Part 1 of 3 )

\begin{tabular}{|c|c|c|c|c|c|c|}
\hline Torsion/Dihedral Type & $\begin{array}{c}K_{1 \phi} \\
(\mathrm{kcal} / \mathrm{mol})\end{array}$ & $\begin{array}{c}\gamma_{1} \\
(\operatorname{deg})\end{array}$ & $\begin{array}{c}K_{2 \phi} \\
(\mathrm{kcal} / \mathrm{mol})\end{array}$ & $\begin{array}{c}\gamma_{2} \\
(\mathrm{deg})\end{array}$ & $\begin{array}{c}K_{3 \phi} \\
(\mathrm{kcal} / \mathrm{mol})\end{array}$ & $\begin{array}{c}\gamma_{3} \\
(\operatorname{deg})\end{array}$ \\
\hline $\mathrm{C} 1-\mathrm{C} 2-\mathrm{C} 3-\mathrm{C} 4^{24}$ & 0.45 & 180 & 0 & 0 & 0 & 0 \\
\hline $\mathrm{C} 1-\mathrm{C} 2-\mathrm{C} 3-\mathrm{H} 3^{24}$ & 0 & 0 & 0 & 0 & 0.15 & 180 \\
\hline $\mathrm{C} 1-\mathrm{C} 2-\mathrm{C} 3-\mathrm{O} 3^{24}$ & 0 & 0 & 0 & 0 & 0.10 & 180 \\
\hline $\mathrm{C} 1-\mathrm{C} 2-\mathrm{O} 2-\mathrm{HO}_{2}{ }^{24}$ & 0 & 0 & 0 & 0 & 0.18 & 180 \\
\hline $\mathrm{C} 2-\mathrm{C} 1-\mathrm{O} 1-\mathrm{HO}^{24}$ & 0 & 0 & 0 & 0 & 0.18 & 180 \\
\hline $\mathrm{C} 2-\mathrm{C} 1-\mathrm{O} 4-\mathrm{C} 44^{24}$ & 0 & 0 & 0 & 0 & 0.16 & 180 \\
\hline $\mathrm{C} 2-\mathrm{C} 1-\mathrm{O} 5-\mathrm{C} 5^{24}$ & 0 & 0 & 0 & 0 & 0.16 & 180 \\
\hline $\mathrm{C} 2-\mathrm{C} 3-\mathrm{C} 4-\mathrm{C} 5^{24}$ & 0.45 & 180 & 0 & 0 & 0 & 0 \\
\hline $\mathrm{C} 2-\mathrm{C} 3-\mathrm{C} 4-\mathrm{H} 4^{24}$ & 0 & 0 & 0 & 0 & 0.15 & 180 \\
\hline $\mathrm{C} 2-\mathrm{C} 3-\mathrm{C} 4-\mathrm{O} 4^{24}$ & -0.27 & 180 & 0 & 0 & 0 & 0 \\
\hline $\mathrm{C} 2-\mathrm{C} 3-\mathrm{C} 4-\mathrm{O} 4 \mathrm{~B}^{24}$ & 0 & 0 & 0 & 0 & 0.10 & 180 \\
\hline $\mathrm{C} 2-\mathrm{C} 3-\mathrm{O} 3-\mathrm{HO}^{24}$ & 0 & 0 & 0 & 0 & 0.18 & 180 \\
\hline $\mathrm{C} 3-\mathrm{C} 2-\mathrm{O} 2-\mathrm{HO}_{2}{ }^{24}$ & 0 & 0 & 0 & 0 & 0.18 & 180 \\
\hline $\mathrm{C} 3-\mathrm{C} 4-\mathrm{C} 5-\mathrm{C} 6{ }^{24}$ & 0.45 & 180 & 0 & 0 & 0 & 0 \\
\hline $\mathrm{C} 3-\mathrm{C} 4-\mathrm{C} 5-\mathrm{H} 5^{24}$ & 0 & 0 & 0 & 0 & 0.15 & 180 \\
\hline $\mathrm{C} 3-\mathrm{C} 4-\mathrm{C} 5-\mathrm{O} 5^{24}$ & -0.27 & 180 & 0 & 0 & 0 & 0 \\
\hline $\mathrm{C} 3-\mathrm{C} 4-\mathrm{O} 4-\mathrm{C} 1^{24}$ & 0 & 0 & 0 & 0 & 0.16 & 180 \\
\hline C3-C4-O4B-HO4 ${ }^{24}$ & 0 & 0 & 0 & 0 & 0.18 & 180 \\
\hline $\mathrm{C} 4-\mathrm{C} 3-\mathrm{O} 3-\mathrm{HO}^{24}$ & 0 & 0 & 0 & 0 & 0.18 & 180 \\
\hline $\mathrm{C} 4-\mathrm{C} 5-\mathrm{C} 6-\mathrm{H} 61^{24}$ & 0 & 0 & 0 & 0 & 0.15 & 180 \\
\hline $\mathrm{C} 4-\mathrm{C} 5-\mathrm{C} 6-\mathrm{H} 62^{24}$ & 0 & 0 & 0 & 0 & 0.15 & 180 \\
\hline $\mathrm{C} 4-\mathrm{C} 5-\mathrm{C} 6-\mathrm{O} 6^{24}$ & 0 & 0 & 0 & 0 & 0.10 & 180 \\
\hline $\mathrm{C} 4-\mathrm{C} 5-\mathrm{O} 5-\mathrm{C} 1^{24}$ & 0 & 0 & 0 & 0 & 0.16 & 180 \\
\hline $\mathrm{C} 5-\mathrm{C} 4-\mathrm{O} 4-\mathrm{C} 1^{24}$ & 0 & 0 & 0 & 0 & 0.16 & 180 \\
\hline $\mathrm{C} 5-\mathrm{C} 4-\mathrm{O} 4 \mathrm{~B}-\mathrm{HO} 4^{24}$ & 0 & 0 & 0 & 0 & 0.18 & 180 \\
\hline $\mathrm{C} 5-\mathrm{C} 6-\mathrm{O} 6-\mathrm{HO}^{24}$ & 0 & 0 & 0 & 0 & 0.18 & 180 \\
\hline $\mathrm{C} 6-\mathrm{C} 5-\mathrm{O} 5-\mathrm{C} 1^{24}$ & 0 & 0 & 0 & 0 & 0.16 & 180 \\
\hline CT1-CT2-CT3-CT4 ${ }^{36}$ & 0 & 0 & 0 & 0 & 0.089930 & 180 \\
\hline $\mathrm{CT} 1-\mathrm{CT} 2-\mathrm{CT} 3-\mathrm{HC} 3^{36}$ & 0 & 0 & 0 & 0 & 0.079894 & 180 \\
\hline CT2-CT1-P-CT1 ${ }^{36}$ & 0 & 0 & 0 & 0 & 0.082402 & 180 \\
\hline CT2-CT3-CT4-HC4 $4^{36}$ & 0 & 0 & 0 & 0 & 0.079894 & 180 \\
\hline
\end{tabular}


Table (7.8) Torsion/dihedral angle coefficients (Part 2 of 3 )

\begin{tabular}{|c|c|c|c|c|c|c|}
\hline Torsion/Dihedral Type & $\begin{array}{c}K_{1 \phi} \\
(\mathrm{kcal} / \mathrm{mol})\end{array}$ & $\begin{array}{c}\gamma_{1} \\
(\operatorname{deg})\end{array}$ & $\begin{array}{c}K_{2 \phi} \\
(\mathrm{kcal} / \mathrm{mol})\end{array}$ & $\begin{array}{c}\gamma_{2} \\
(\operatorname{deg})\end{array}$ & $\begin{array}{c}K_{3 \phi} \\
(\mathrm{kcal} / \mathrm{mol})\end{array}$ & $\begin{array}{c}\gamma_{3} \\
(\mathrm{deg})\end{array}$ \\
\hline $\mathrm{H} 1-\mathrm{C} 1-\mathrm{C} 2-\mathrm{C} 3^{24}$ & 0 & 0 & 0 & 0 & 0.15 & 180 \\
\hline $\mathrm{H} 1-\mathrm{C} 1-\mathrm{C} 2-\mathrm{H} 2^{24}$ & 0 & 0 & 0 & 0 & 0.17 & 180 \\
\hline $\mathrm{H} 1-\mathrm{C} 1-\mathrm{C} 2-\mathrm{O} 2^{24}$ & 0 & 0 & 0 & 0 & 0.05 & 180 \\
\hline $\mathrm{H} 1-\mathrm{C} 1-\mathrm{O} 1-\mathrm{HO}^{24}$ & 0 & 0 & 0 & 0 & 0.18 & 180 \\
\hline $\mathrm{H} 1-\mathrm{C} 1-\mathrm{O} 4-\mathrm{C} 44^{24}$ & 0 & 180 & 0.6 & 180 & 0.1 & 180 \\
\hline $\mathrm{H} 1-\mathrm{C} 1-\mathrm{O} 5-\mathrm{C} 5^{24}$ & 0 & 180 & 0.6 & 180 & 0.1 & 180 \\
\hline $\mathrm{H} 2-\mathrm{C} 2-\mathrm{C} 3-\mathrm{C} 4{ }^{24}$ & 0 & 0 & 0 & 0 & 0.15 & 180 \\
\hline $\mathrm{H} 2-\mathrm{C} 2-\mathrm{C} 3-\mathrm{H} 3^{24}$ & 0 & 0 & 0 & 0 & 0.17 & 180 \\
\hline $\mathrm{H} 2-\mathrm{C} 2-\mathrm{C} 3-\mathrm{O} 3{ }^{24}$ & 0 & 0 & 0 & 0 & 0.05 & 180 \\
\hline $\mathrm{H} 2-\mathrm{C} 2-\mathrm{O} 2-\mathrm{HO}_{2}{ }^{24}$ & 0 & 0 & 0 & 0 & 0.18 & 180 \\
\hline H3-C3-C4-C5 ${ }^{24}$ & 0 & 0 & 0 & 0 & 0.15 & 180 \\
\hline $\mathrm{H} 3-\mathrm{C} 3-\mathrm{C} 4-\mathrm{H} 4^{24}$ & 0 & 0 & 0 & 0 & 0.17 & 180 \\
\hline $\mathrm{H} 3-\mathrm{C} 3-\mathrm{C} 4-\mathrm{O} 4{ }^{24}$ & 0 & 0 & 0 & 0 & 0.05 & 180 \\
\hline $\mathrm{H} 3-\mathrm{C} 3-\mathrm{C} 4-\mathrm{O} 4 \mathrm{~B}^{24}$ & 0 & 0 & 0 & 0 & 0.05 & 180 \\
\hline $\mathrm{H} 3-\mathrm{C} 3-\mathrm{O} 3-\mathrm{HO}^{24}$ & 0 & 0 & 0 & 0 & 0.18 & 180 \\
\hline $\mathrm{H} 4-\mathrm{C} 4-\mathrm{C} 5-\mathrm{C} 6^{24}$ & 0 & 0 & 0 & 0 & 0.15 & 180 \\
\hline $\mathrm{H} 4-\mathrm{C} 4-\mathrm{C} 5-\mathrm{H} 5^{24}$ & 0 & 0 & 0 & 0 & 0.17 & 180 \\
\hline $\mathrm{H} 4-\mathrm{C} 4-\mathrm{C} 5-\mathrm{O} 5^{24}$ & 0 & 0 & 0 & 0 & 0.05 & 180 \\
\hline $\mathrm{H} 4-\mathrm{C} 4-\mathrm{O} 4-\mathrm{C}^{24}$ & 0 & 0 & 0 & 0 & 0.27 & 180 \\
\hline $\mathrm{H} 4-\mathrm{C} 4-\mathrm{O} 4 \mathrm{~B}-\mathrm{HO} 4^{24}$ & 0 & 0 & 0 & 0 & 0.18 & 180 \\
\hline $\mathrm{H} 5-\mathrm{C} 5-\mathrm{C} 6-\mathrm{H} 61^{24}$ & 0 & 0 & 0 & 0 & 0.17 & 180 \\
\hline $\mathrm{H} 5-\mathrm{C} 5-\mathrm{C} 6-\mathrm{H} 62^{24}$ & 0 & 0 & 0 & 0 & 0.17 & 180 \\
\hline $\mathrm{H} 5-\mathrm{C} 5-\mathrm{C} 6-\mathrm{O}^{24}$ & 0 & 0 & 0 & 0 & 0.05 & 180 \\
\hline $\mathrm{H} 5-\mathrm{C} 5-\mathrm{O} 5-\mathrm{C} 1^{24}$ & 0 & 0 & 0 & 0 & 0.27 & 180 \\
\hline $\mathrm{H} 61-\mathrm{C} 6-\mathrm{O} 6-\mathrm{HO}^{24}$ & 0 & 0 & 0 & 0 & 0.18 & 180 \\
\hline $\mathrm{H} 62-\mathrm{C} 6-\mathrm{O} 6-\mathrm{HO}^{24}$ & 0 & 0 & 0 & 0 & 0.18 & 180 \\
\hline $\mathrm{HC} 2-\mathrm{CT} 2-\mathrm{CT} 3-\mathrm{CT} 4^{36}$ & 0 & 0 & 0 & 0 & 0.079894 & 180 \\
\hline HC2-CT2-CT3-HC3 ${ }^{36}$ & 0 & 0 & 0 & 0 & 0.074998 & 180 \\
\hline $\mathrm{HC} 3-\mathrm{CT} 3-\mathrm{CT} 4-\mathrm{HC} 4^{36}$ & 0 & 0 & 0 & 0 & 0.074998 & 180 \\
\hline HP-CT1-CT2-CT $3^{36}$ & 0 & 0 & 0 & 0 & 0.079894 & 180 \\
\hline $\mathrm{HP}-\mathrm{CT} 1-\mathrm{CT} 2-\mathrm{HC} 2^{36}$ & 0 & 0 & 0 & 0 & 0.074998 & 180 \\
\hline HP-CT1-P-CT1 $1^{36}$ & 0 & 0 & 0 & 0 & 0.082402 & 180 \\
\hline
\end{tabular}


Table (7.9) Torsion/dihedral angle coefficients (Part 3 of 3)

\begin{tabular}{|c|c|c|c|c|c|c|}
\hline Torsion/Dihedral Type & $\begin{array}{c}K_{1 \phi} \\
(\mathrm{kcal} / \mathrm{mol})\end{array}$ & $\begin{array}{c}\gamma_{1} \\
(\mathrm{deg})\end{array}$ & $\begin{array}{c}K_{2 \phi} \\
(\mathrm{kcal} / \mathrm{mol})\end{array}$ & $\begin{array}{c}\gamma_{2} \\
(\mathrm{deg})\end{array}$ & $\begin{array}{c}K_{3 \phi} \\
(\mathrm{kcal} / \mathrm{mol})\end{array}$ & $\begin{array}{c}\gamma_{3} \\
(\mathrm{deg})\end{array}$ \\
\hline $\mathrm{O} 1-\mathrm{C} 1-\mathrm{C} 2-\mathrm{C}^{24}$ & 0 & 0 & 0 & 0 & 0.10 & 180 \\
\hline $\mathrm{O} 1-\mathrm{C} 1-\mathrm{C} 2-\mathrm{H} 2^{24}$ & 0 & 0 & 0 & 0 & 0.05 & 180 \\
\hline $\mathrm{O} 1-\mathrm{C} 1-\mathrm{C} 2-\mathrm{O} 2{ }^{24}$ & -0.1 & 180 & 0.95 & 180 & 0.55 & 180 \\
\hline $\mathrm{O} 1-\mathrm{C} 1-\mathrm{O} 5-\mathrm{C} 5^{24}$ & 1.08 & 180 & 1.38 & 180 & 0.96 & 180 \\
\hline $\mathrm{O} 2-\mathrm{C} 2-\mathrm{C} 3-\mathrm{C} 4{ }^{24}$ & 0 & 0 & 0 & 0 & 0.10 & 180 \\
\hline $\mathrm{O} 2-\mathrm{C} 2-\mathrm{C} 3-\mathrm{H} 3^{24}$ & 0 & 0 & 0 & 0 & 0.05 & 180 \\
\hline $\mathrm{O} 2-\mathrm{C} 2-\mathrm{C} 3-\mathrm{O} 3{ }^{24}$ & -0.1 & 180 & 0.95 & 180 & 0.55 & 180 \\
\hline O3-C3-C4-C5 ${ }^{24}$ & 0 & 0 & 0 & 0 & 0.10 & 180 \\
\hline $\mathrm{O} 3-\mathrm{C} 3-\mathrm{C} 4-\mathrm{H} 4^{24}$ & 0 & 0 & 0 & 0 & 0.05 & 180 \\
\hline $\mathrm{O} 3-\mathrm{C} 3-\mathrm{C} 4-\mathrm{O} 4^{24}$ & -1.1 & 180 & 0.25 & 180 & 0 & 180 \\
\hline $\mathrm{O} 3-\mathrm{C} 3-\mathrm{C} 4-\mathrm{O} 4 \mathrm{~B}^{24}$ & -0.1 & 180 & 0.95 & 180 & 0.55 & 180 \\
\hline $\mathrm{O} 4-\mathrm{C} 1-\mathrm{C} 2-\mathrm{C} 3^{24}$ & -0.27 & 180 & 0 & 0 & 0 & 0 \\
\hline $\mathrm{O} 4-\mathrm{C} 1-\mathrm{C} 2-\mathrm{H} 2^{24}$ & 0 & 0 & 0 & 0 & 0.05 & 180 \\
\hline $\mathrm{O} 4-\mathrm{C} 1-\mathrm{C} 2-\mathrm{O} 2{ }^{24}$ & -1.1 & 180 & 0.25 & 180 & 0 & 180 \\
\hline $\mathrm{O} 4-\mathrm{C} 1-\mathrm{O} 5-\mathrm{C} 5^{24}$ & 1.08 & 180 & 1.38 & 180 & 0.96 & 180 \\
\hline $\mathrm{O} 4-\mathrm{C} 4-\mathrm{C} 5-\mathrm{C} 6^{24}$ & -0.27 & 180 & 0 & 0 & 0 & 0 \\
\hline $\mathrm{O} 4-\mathrm{C} 4-\mathrm{C} 5-\mathrm{H} 5^{24}$ & 0 & 0 & 0 & 0 & 0.05 & 180 \\
\hline $\mathrm{O} 4-\mathrm{C} 4-\mathrm{C} 5-\mathrm{O} 5^{24}$ & 0 & 0 & 0.4 & 180 & 0 & 0 \\
\hline $\mathrm{O} 4 \mathrm{~B}-\mathrm{C} 4-\mathrm{C} 5-\mathrm{C} 6^{24}$ & 0 & 0 & 0 & 0 & 0.10 & 180 \\
\hline $\mathrm{O} 4 \mathrm{~B}-\mathrm{C} 4-\mathrm{C} 5-\mathrm{H} 5^{24}$ & 0 & 0 & 0 & 0 & 0.05 & 180 \\
\hline $\mathrm{O} 4 \mathrm{~B}-\mathrm{C} 4-\mathrm{C} 5-\mathrm{O} 5^{24}$ & -1.1 & 180 & 0.25 & 180 & 0 & 180 \\
\hline $\mathrm{O} 5-\mathrm{C} 1-\mathrm{C} 2-\mathrm{C} 3^{24}$ & -0.27 & 180 & 0 & 0 & 0 & 0 \\
\hline $\mathrm{O} 5-\mathrm{C} 1-\mathrm{C} 2-\mathrm{H} 2^{24}$ & 0 & 0 & 0 & 0 & 0.05 & 180 \\
\hline $\mathrm{O} 5-\mathrm{C} 1-\mathrm{C} 2-\mathrm{O} 2^{24}$ & -1.1 & 180 & 0.25 & 180 & 0 & 180 \\
\hline $\mathrm{O} 5-\mathrm{C} 1-\mathrm{O} 1-\mathrm{HO}^{24}$ & 0 & 0 & 0 & 0 & 0.18 & 180 \\
\hline $\mathrm{O} 5-\mathrm{C} 1-\mathrm{O} 4-\mathrm{C} 4^{24}$ & 1.08 & 180 & 1.38 & 180 & 0.96 & 180 \\
\hline $\mathrm{O} 5-\mathrm{C} 5-\mathrm{C} 6-\mathrm{H} 61^{24}$ & 0 & 0 & 0 & 0 & 0.05 & 180 \\
\hline $\mathrm{O} 5-\mathrm{C} 5-\mathrm{C} 6-\mathrm{H} 62^{24}$ & 0 & 0 & 0 & 0 & 0.05 & 180 \\
\hline $\mathrm{O} 5-\mathrm{C} 5-\mathrm{C} 6-\mathrm{O} 6^{24}$ & -1.1 & 180 & 0.25 & 180 & 0 & 180 \\
\hline $\mathrm{P}-\mathrm{CT} 1-\mathrm{CT} 2-\mathrm{CT} 3^{36}$ & 0 & 0 & 0 & 0 & 0.072012 & 180 \\
\hline $\mathrm{P}-\mathrm{CT} 1-\mathrm{CT} 2-\mathrm{HC} 2^{36}$ & 0 & 0 & 0 & 0 & 0.072012 & 180 \\
\hline
\end{tabular}




\subsection{Small and Large $\mathrm{I} \beta$ Bundle Comparison in TBPCl-water (cont.)}

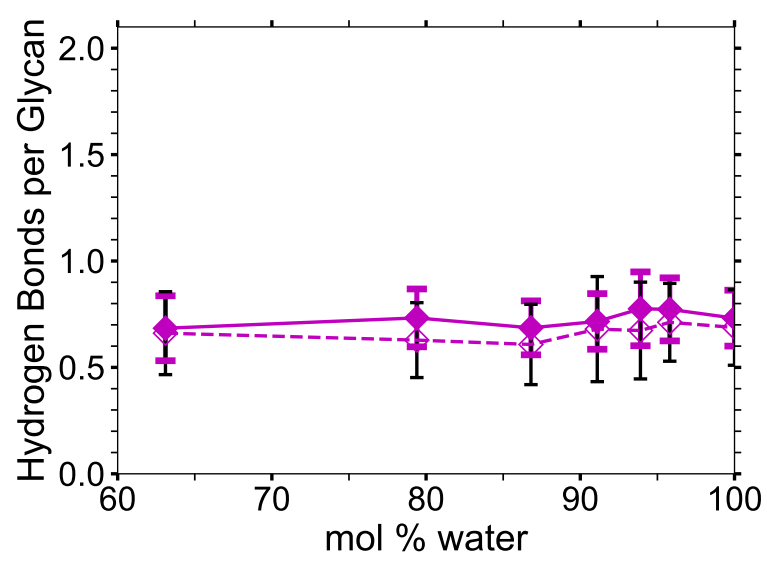

(a) First layer strands

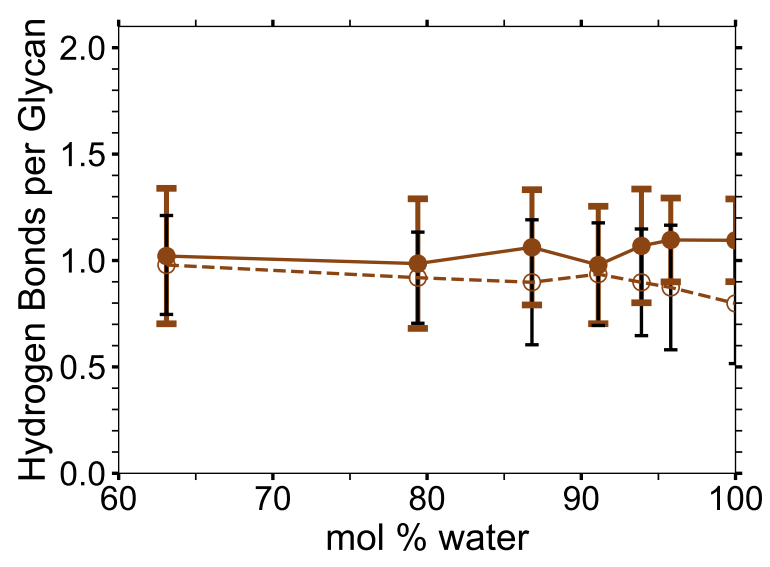

(c) Second layer (outer) strands

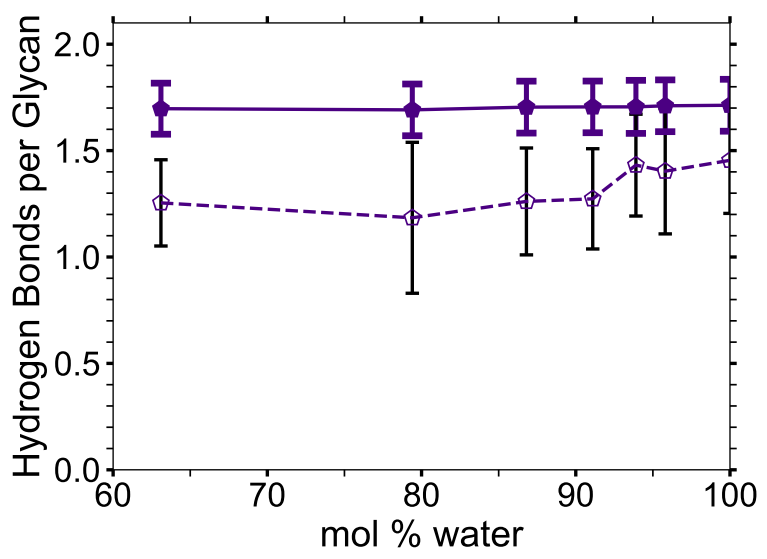

(b) Center strands

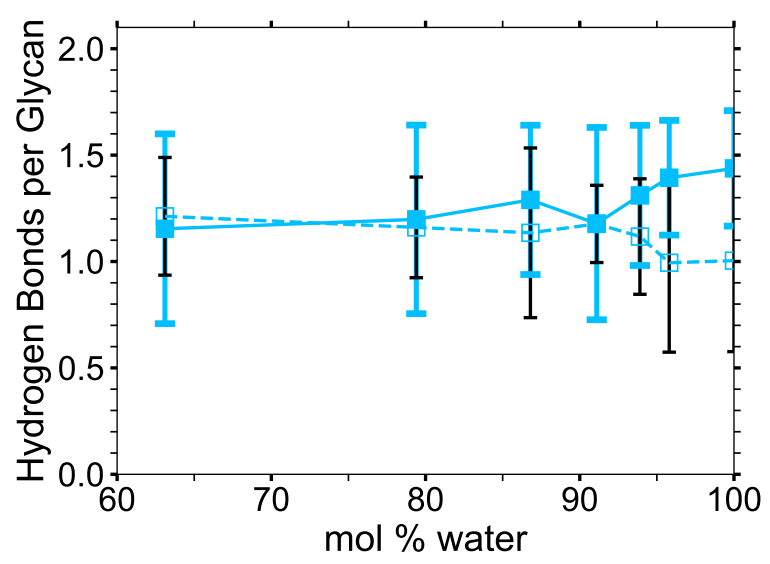

(d) Second layer (inner) strands

Figure (7.1) Average intra-strand hydrogen bonding comparison with standard deviations between the small and large cellulose bundles at various water concentrations (Part 1 of 2): (a) the first layer strands; (b) the center strands; (c) the second layer (outer) strands; (d) the second layer (inner) strands. All data points were averaged from 10 to 20 ns. The solid lines, filled markers, and colored error bars represent the large cellulose bundle, and the dashed lines, unfilled marker, and black error bars show the small cellulose bundle. The colored strands are matched and compared between the plots for the small and large bundles and the images to identify similar hydrogen bonding behavior based on their locations (see Figures $3.2 \mathrm{a}$ and $3.2 \mathrm{~b}$ for the color-coded images). The black cellulose strands are not compared. The small and large cellulose bundle intra-strand hydrogen bonding is based on 11 glycans and 23 glycans, as the last glycan has no potential bonding partner. ${ }^{22}$ 


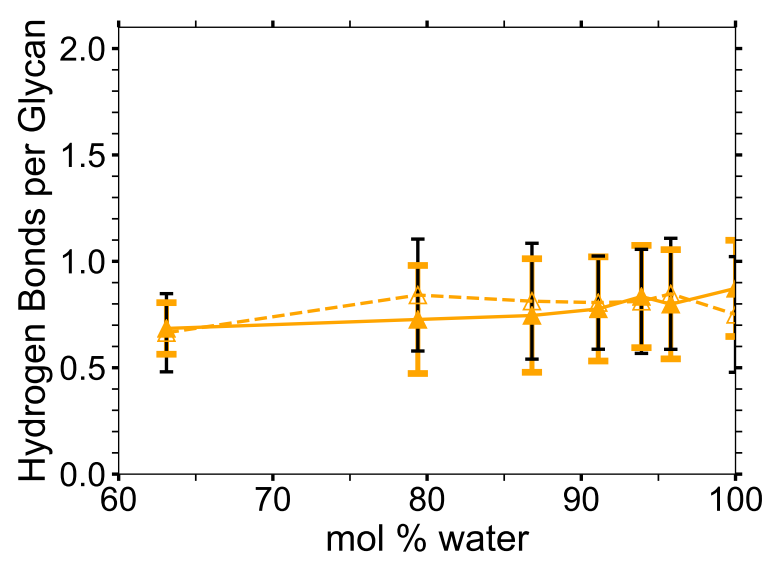

(a) Corner strands

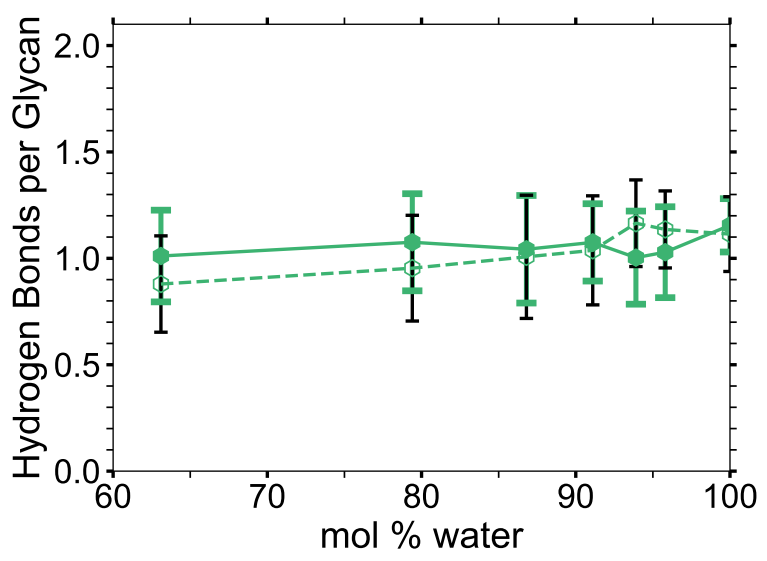

(b) The strands above the corner strands

Figure (7.2) Average intra-strand hydrogen bonding comparison with standard deviations between the small and large cellulose bundles at various water concentrations (Part 2 of 2): (a) the corner strands; (b) the strands above the corner strands. All data points were averaged from 10 to $20 \mathrm{~ns}$. The solid lines, filled markers, and colored error bars represent the large cellulose bundle, and the dashed lines, unfilled marker, and black error bars show the small cellulose bundle. The colored strands are matched and compared between the plots for the small and large bundles and the images to identify similar hydrogen bonding behavior based on their locations (see Figures 3.2a and 3.2b for the color-coded images). The black cellulose strands are not compared. The small and large cellulose bundle intra-strand hydrogen bonding is based on 11 glycans and 23 glycans, as the last glycan has no potential bonding partner. ${ }^{22}$ 


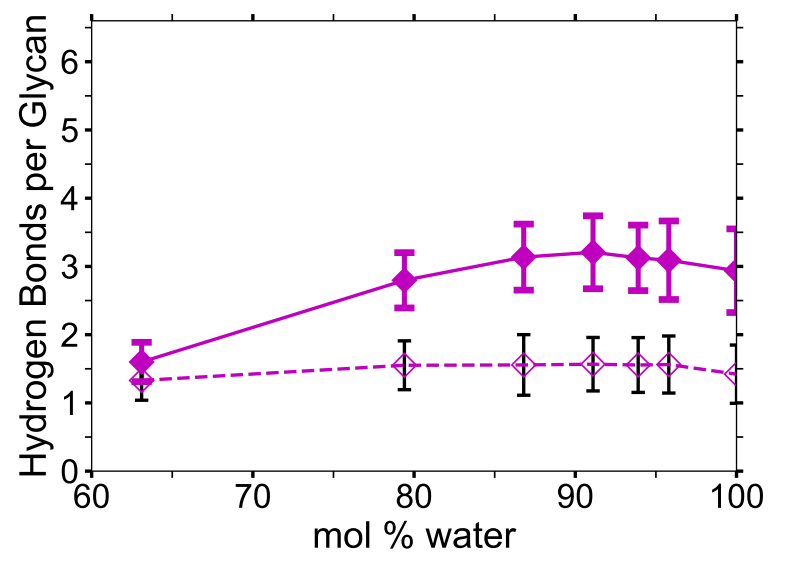

(a) First layer strands

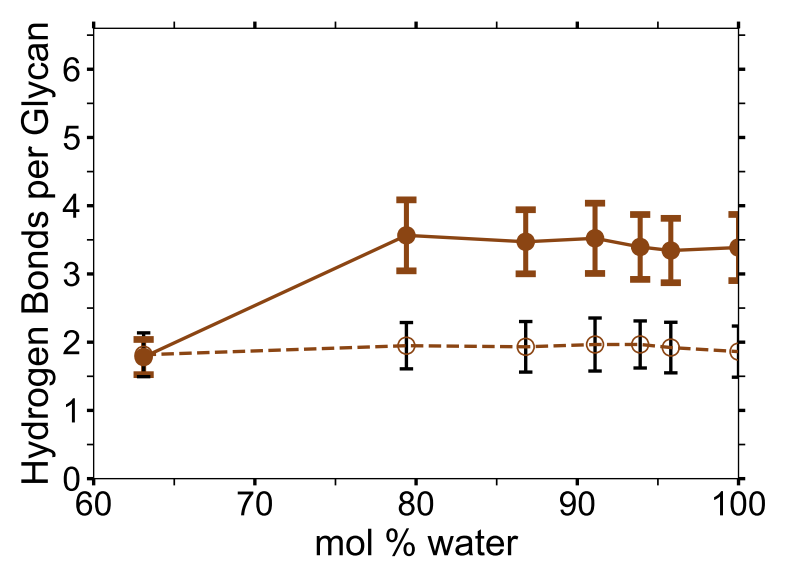

(c) Second layer (outer) strands

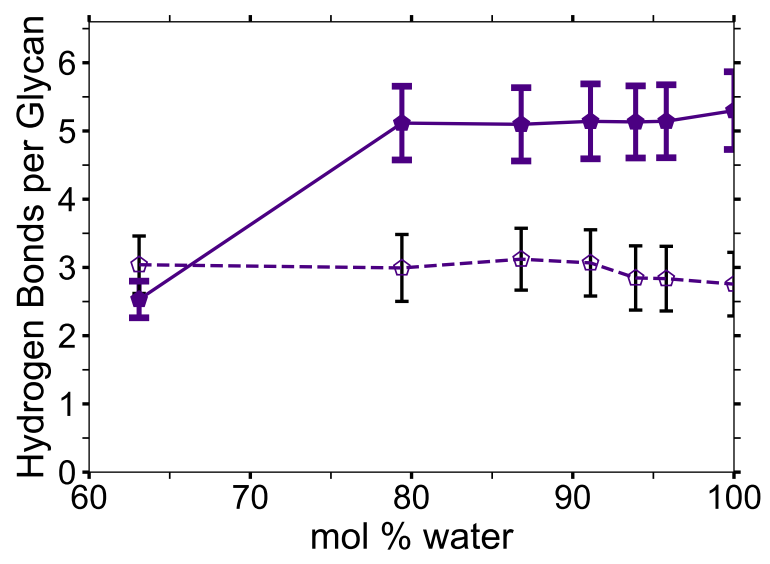

(b) Center strands

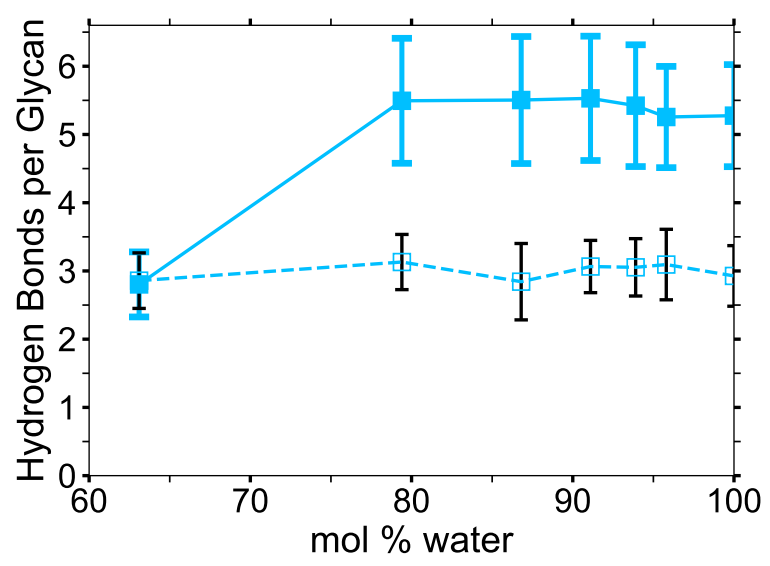

(d) Second layer (inner) strands

Figure (7.3) Average inter-strand hydrogen bonding comparison with standard deviations between the small and large cellulose bundles at various water concentrations (Part 1 of 2): (a) the first layer strands; (b) the center strands; (c) the second layer (outer) strands; (d) the second layer (inner) strands. All data points were averaged from 10 to $20 \mathrm{~ns}$. The solid lines, filled markers, and colored error bars represent the large cellulose bundle, and the dashed lines, unfilled marker, and black error bars show the small cellulose bundle. The colored strands are matched and compared between the plots for the small and large bundles and the images to identify similar hydrogen bonding behavior based on their locations (see Figures $3.2 \mathrm{a}$ and $3.2 \mathrm{~b}$ for the color-coded images). The black cellulose strands are not compared. $^{22}$ 


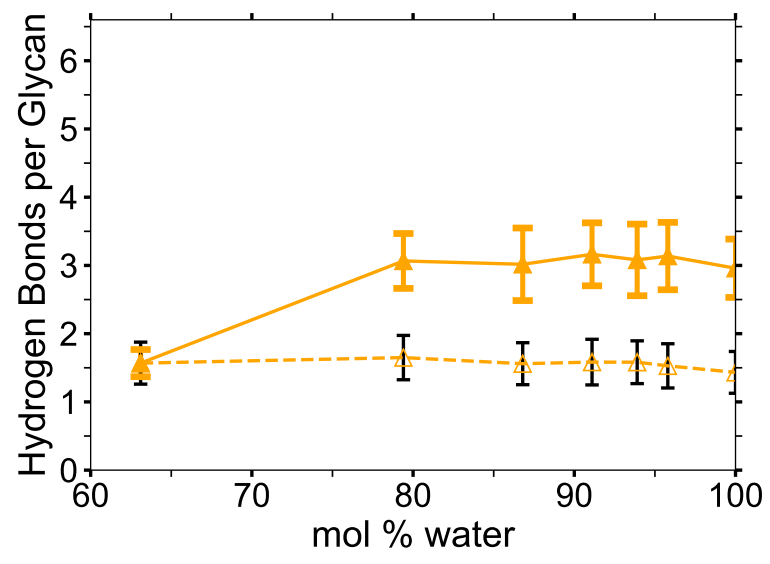

(a) Corner strands

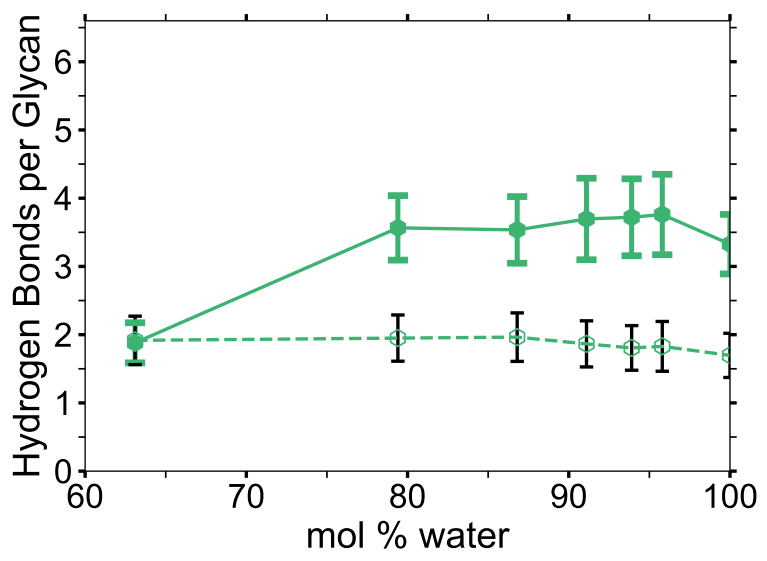

(b) The strands above the corner strands

Figure (7.4) Average inter-strand hydrogen bonding comparison with standard deviations between the small and large cellulose bundles at various water concentrations (Part 2 of 2): (a) the corner strands; (b) the strands above the corner strands. All data points were averaged from 10 to $20 \mathrm{~ns}$. The solid lines, filled markers, and colored error bars represent the large cellulose bundle, and the dashed lines, unfilled marker, and black error bars show the small cellulose bundle. The colored strands are matched and compared between the plots for the small and large bundles and the images to identify similar hydrogen bonding behavior based on their locations (see Figures $3.2 \mathrm{a}$ and $3.2 \mathrm{~b}$ for the color-coded images). The black cellulose strands are not compared. ${ }^{22}$ 


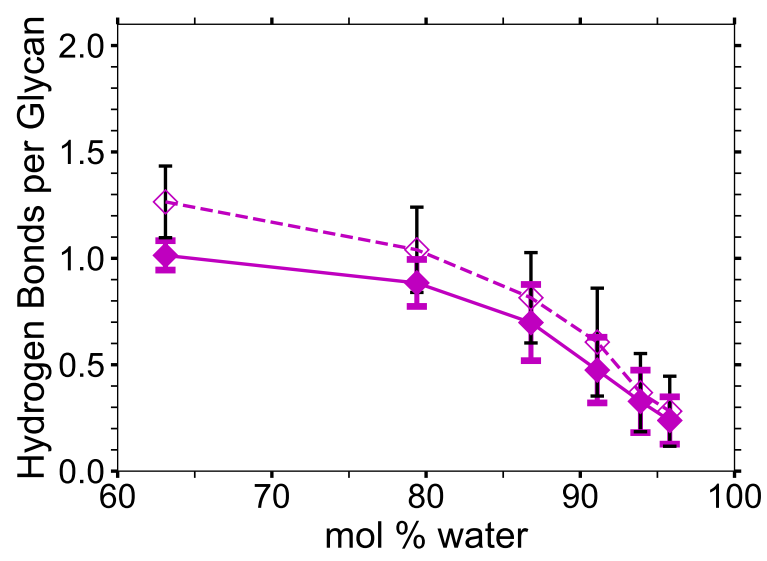

(a) First layer strands

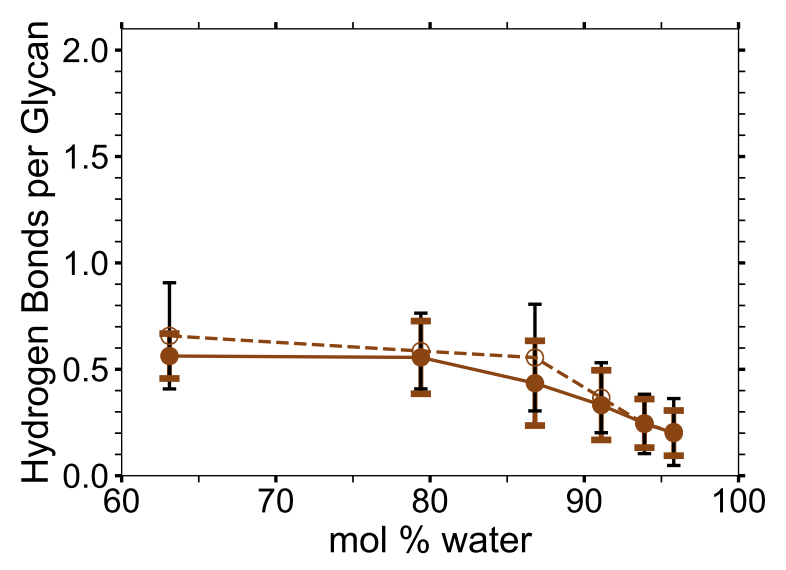

(c) Second layer (outer) strands

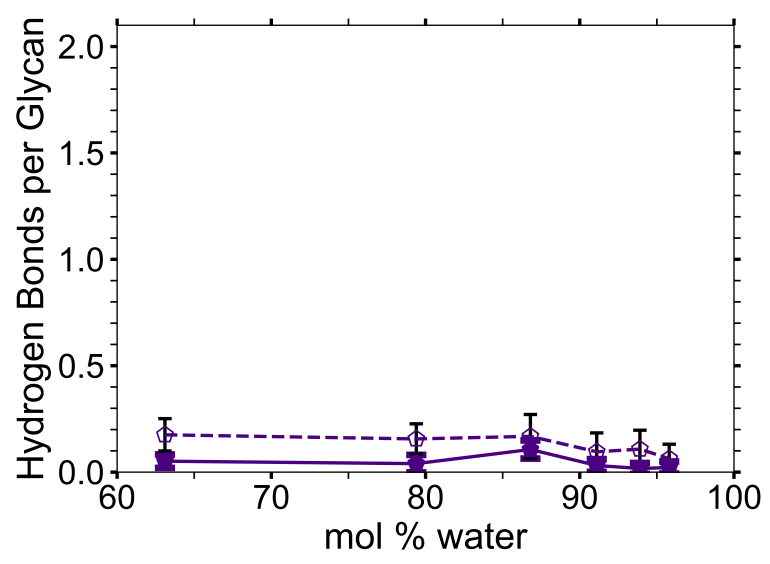

(b) Center strands

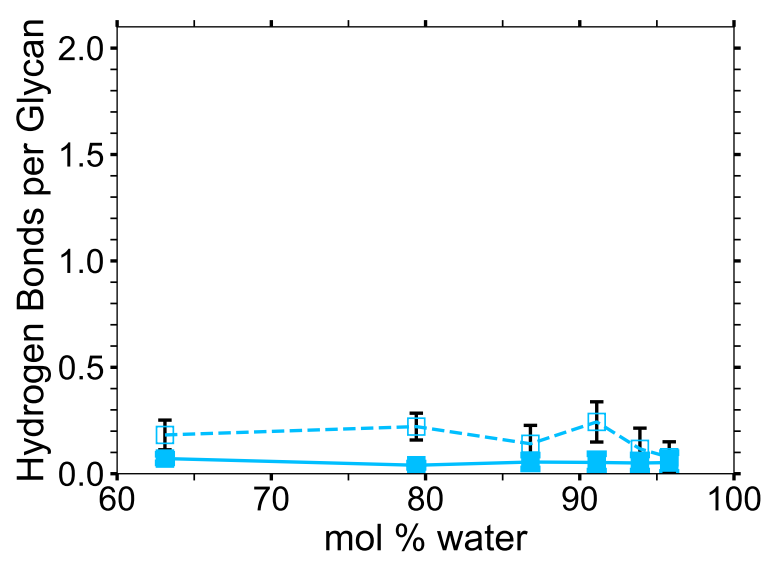

(d) Second layer (inner) strands

Figure (7.5) Average Cl-strand hydrogen bonding comparison with standard deviations between the small and large cellulose bundles at various water concentrations (Part 1 of 2): (a) the first layer strands; (b) the center strands; (c) the second layer (outer) strands; (d) the second layer (inner) strands. All data points were averaged from 10 to $20 \mathrm{~ns}$. The solid lines, filled markers, and colored error bars represent the large cellulose bundle, and the dashed lines, unfilled marker, and black error bars show the small cellulose bundle. The colored strands are matched and compared between the plots for the small and large bundles and the images to identify similar hydrogen bonding behavior based on their locations (see Figures $3.2 \mathrm{a}$ and $3.2 \mathrm{~b}$ for the color-coded images). The black cellulose strands are not compared. ${ }^{22}$ 


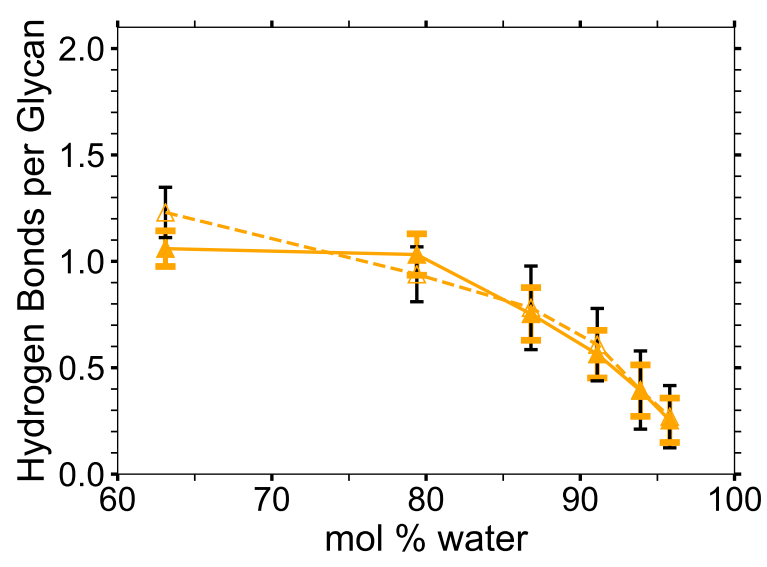

(a) Corner strands

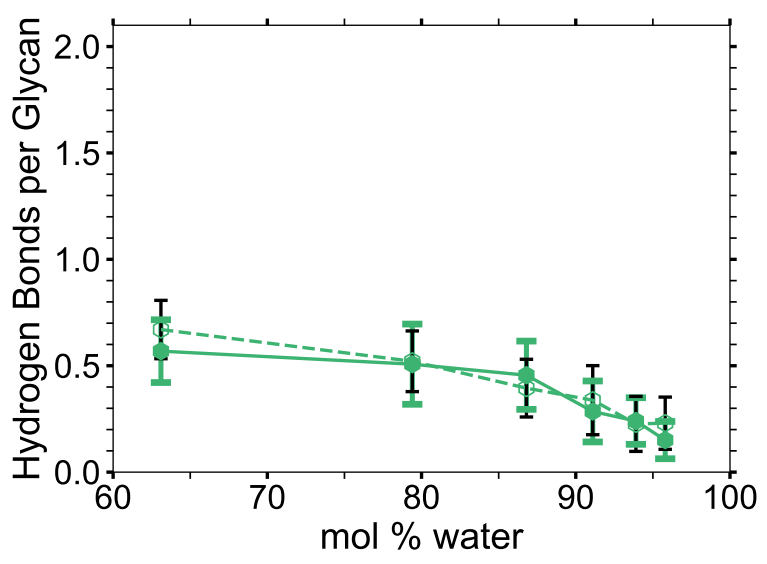

(b) The strands above the corner strands

Figure (7.6) Average Cl-strand hydrogen bonding comparison with standard deviations between the small and large cellulose bundles at various water concentrations (Part 2 of 2): (a) the corner strands; (b) the strands above the corner strands. All data points were averaged from 10 to $20 \mathrm{~ns}$. The solid lines, filled markers, and colored error bars represent the large cellulose bundle, and the dashed lines, unfilled marker, and black error bars show the small cellulose bundle. The colored strands are matched and compared between the plots for the small and large bundles and the images to identify similar hydrogen bonding behavior based on their locations (see Figures 3.2a and 3.2b for the color-coded images). The black cellulose strands are not compared. ${ }^{22}$ 


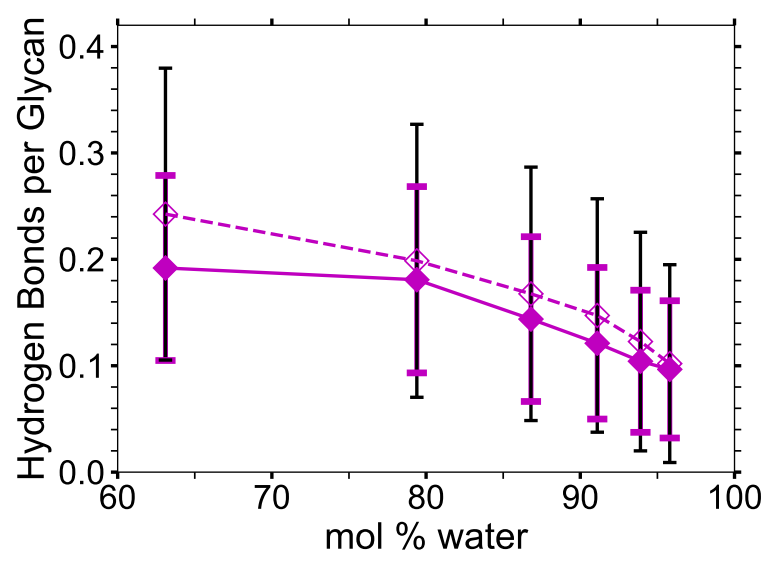

(a) First layer strands

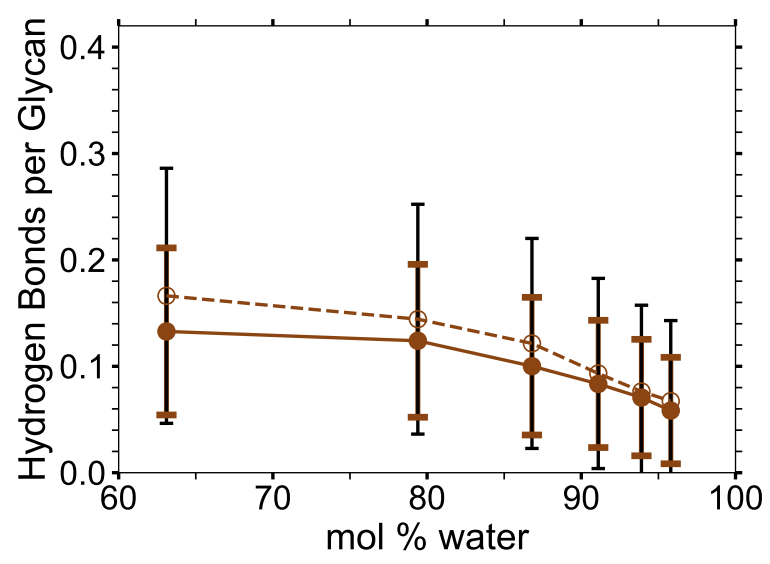

(c) Second layer (outer) strands

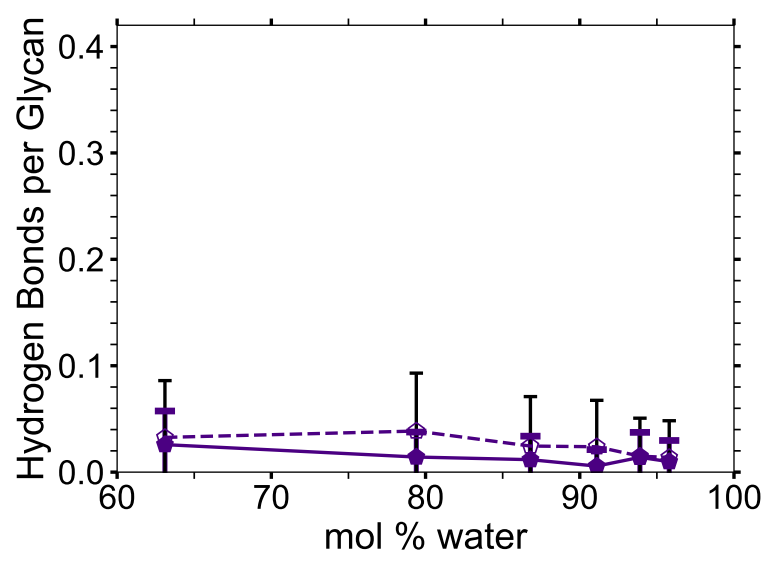

(b) Center strands

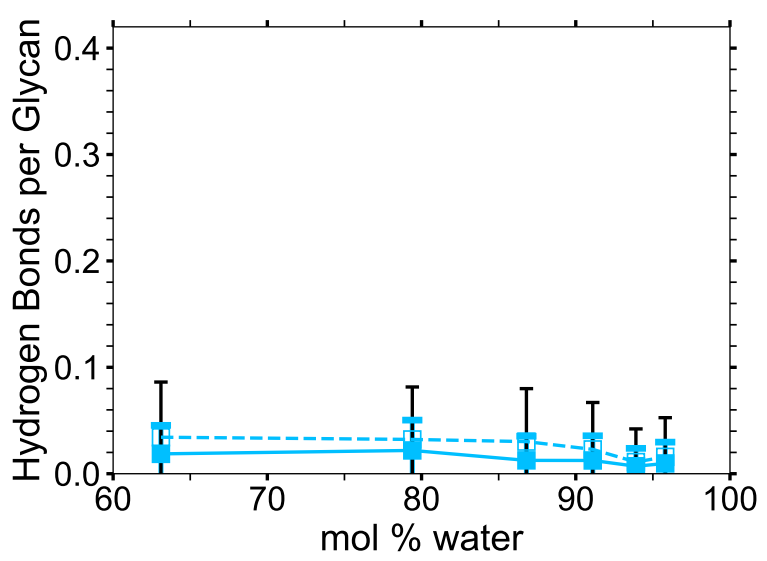

(d) Second layer (inner) strands

Figure (7.7) Average TBP-strand hydrogen bonding comparison with standard deviations between the small and large cellulose bundles at various water concentrations (Part 1 of 2): (a) the first layer strands; (b) the center strands; (c) the second layer (outer) strands; (d) the second layer (inner) strands. All data points were averaged from 10 to $20 \mathrm{~ns}$. The solid lines, filled markers, and colored error bars represent the large cellulose bundle, and the dashed lines, unfilled marker, and black error bars show the small cellulose bundle. The colored strands are matched and compared between the plots for the small and large bundles and the images to identify similar hydrogen bonding behavior based on their locations (see Figures $3.2 \mathrm{a}$ and $3.2 \mathrm{~b}$ for the color-coded images). The black cellulose strands are not compared. ${ }^{22}$ 


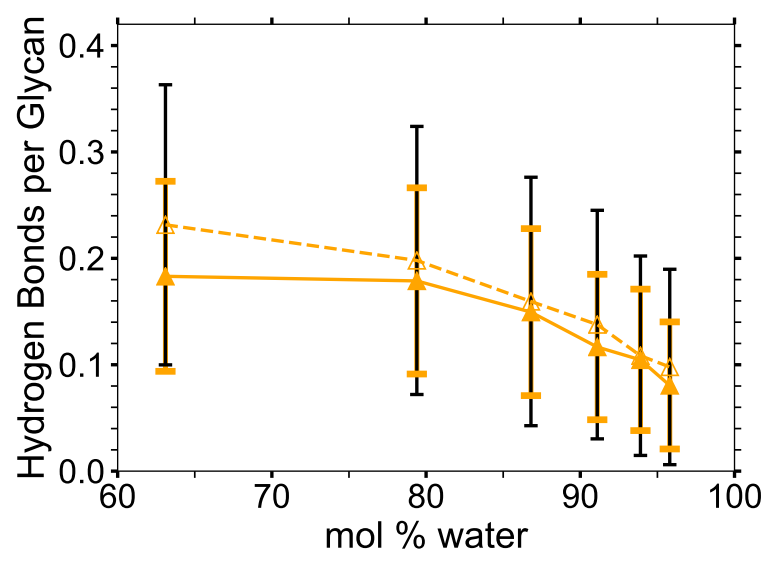

(a) Corner strands

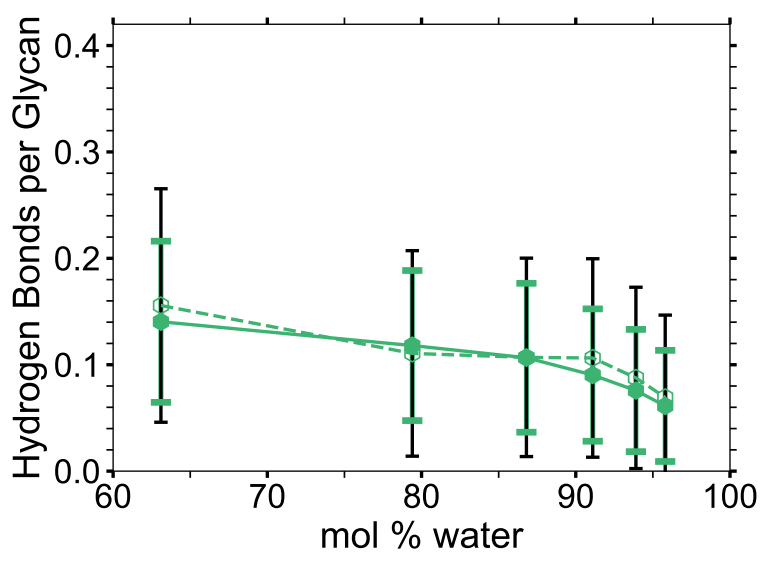

(b) The strands above the corner strands

Figure (7.8) Average TBP-strand hydrogen bonding comparison with standard deviations between the small and large cellulose bundles at various water concentrations (Part 2 of 2): (a) the corner strands; (b) the strands above the corner strands. All data points were averaged from 10 to $20 \mathrm{~ns}$. The solid lines, filled markers, and colored error bars represent the large cellulose bundle, and the dashed lines, unfilled marker, and black error bars show the small cellulose bundle. The colored strands are matched and compared between the plots for the small and large bundles and the images to identify similar hydrogen bonding behavior based on their locations (see Figures $3.2 \mathrm{a}$ and $3.2 \mathrm{~b}$ for the color-coded images). The black cellulose strands are not compared. ${ }^{22}$ 


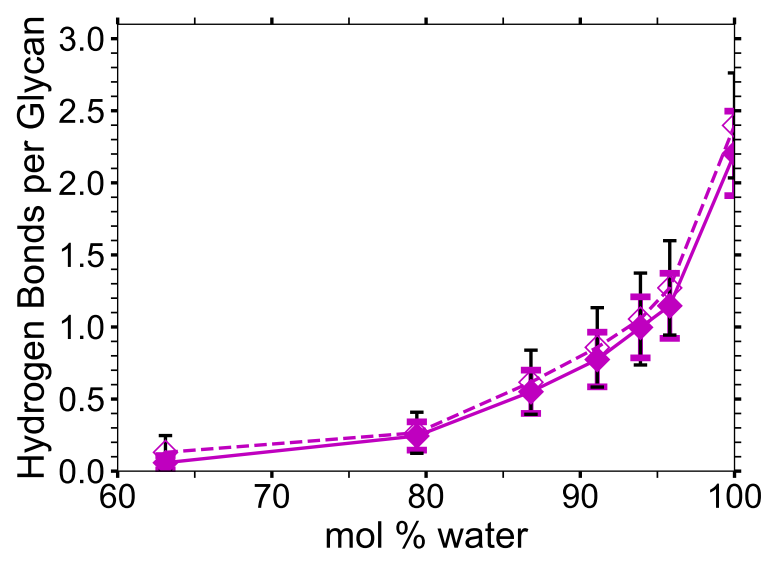

(a) First layer strands

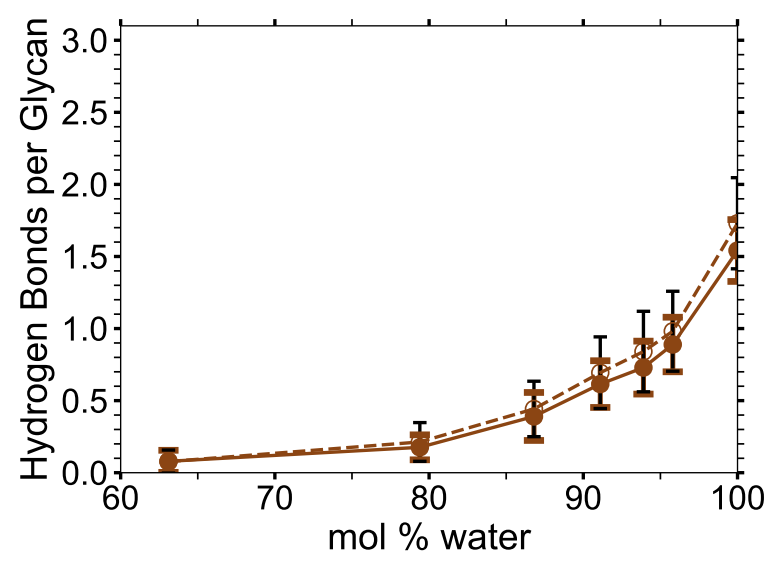

(c) Second layer (outer) strands

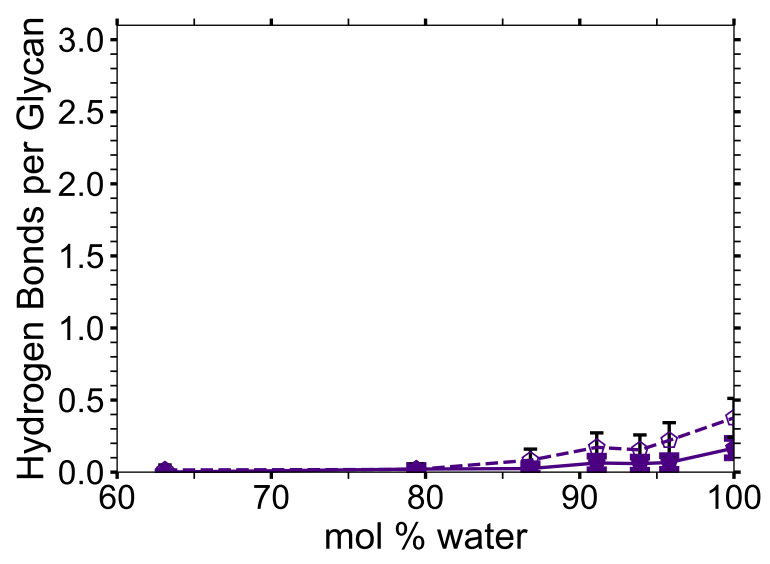

(b) Center strands

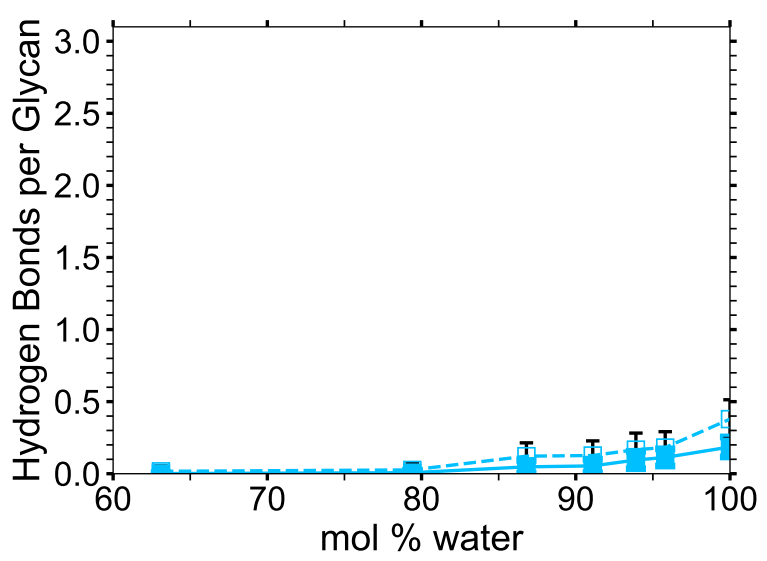

(d) Second layer (inner) strands

Figure (7.9) Average water-strand hydrogen bonding comparison with standard deviations between the small and large cellulose bundles at various water concentrations (Part 1 of 2): (a) the first layer strands; (b) the center strands; (c) the second layer (outer) strands; (d) the second layer (inner) strands. All data points were averaged from 10 to 20 ns. The solid lines, filled markers, and colored error bars represent the large cellulose bundle, and the dashed lines, unfilled marker, and black error bars show the small cellulose bundle. The colored strands are matched and compared between the plots for the small and large bundles and the images to identify similar hydrogen bonding behavior based on their locations (see Figures 3.2a and 3.2b for the color-coded images). The black cellulose strands are not compared. ${ }^{22}$ 


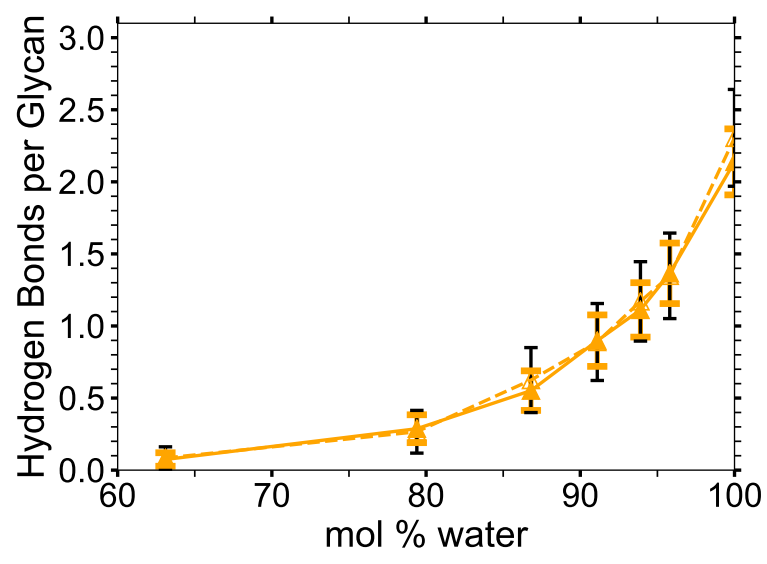

(a) Corner strands

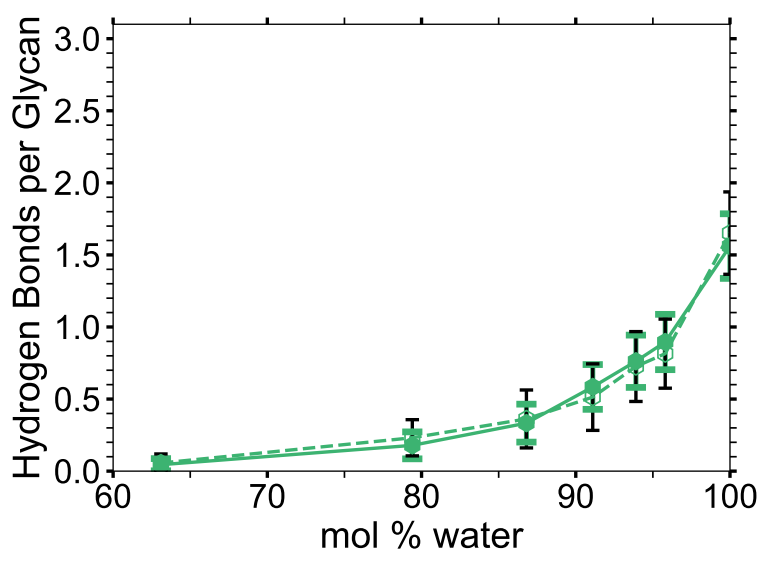

(b) The strands above the corner strands

Figure (7.10) Average water-strand hydrogen bonding comparison with standard deviations between the small and large cellulose bundles at various water concentrations (Part 2 of 2): (a) the corner strands; (b) the strands above the corner strands. All data points were averaged from 10 to $20 \mathrm{~ns}$. The solid lines, filled markers, and colored error bars represent the large cellulose bundle, and the dashed lines, unfilled marker, and black error bars show the small cellulose bundle. The colored strands are matched and compared between the plots for the small and large bundles and the images to identify similar hydrogen bonding behavior based on their locations (see Figures $3.2 \mathrm{a}$ and $3.2 \mathrm{~b}$ for the color-coded images). The black cellulose strands are not compared. ${ }^{22}$ 


\subsection{Radial Distribution Functions (RDFs) for TBPH-water Solu- tion}
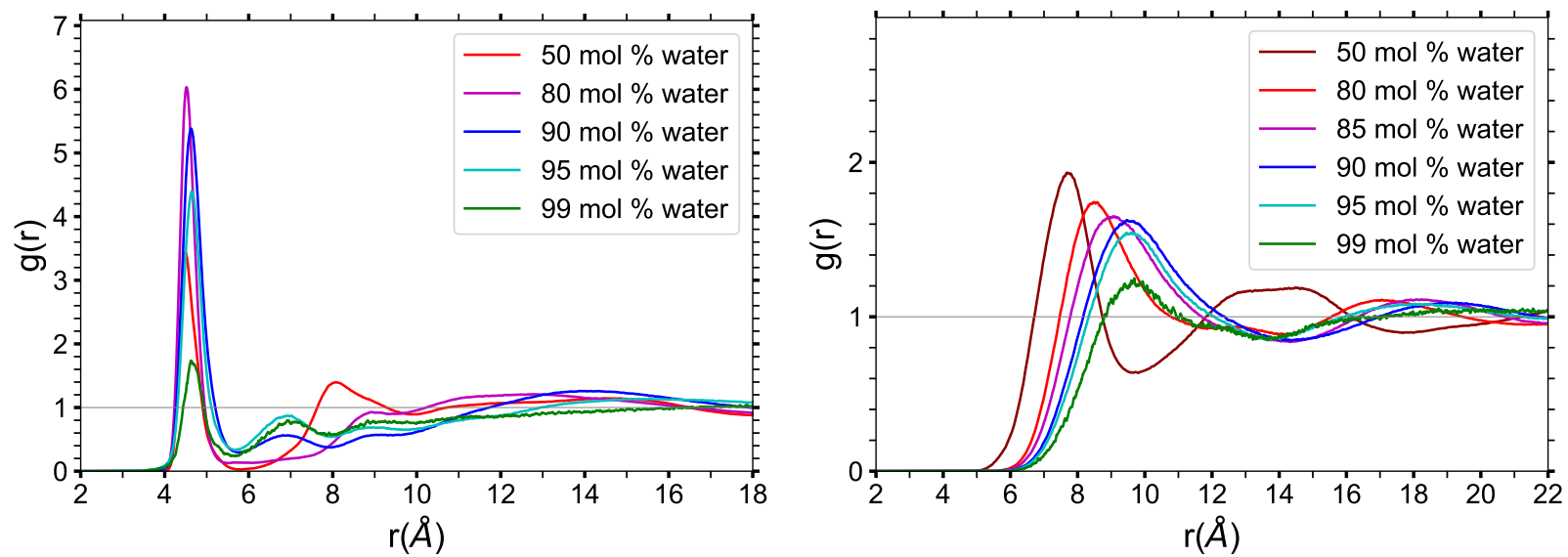

(a) $\mathrm{OH}^{-}-\mathrm{OH}^{-}$

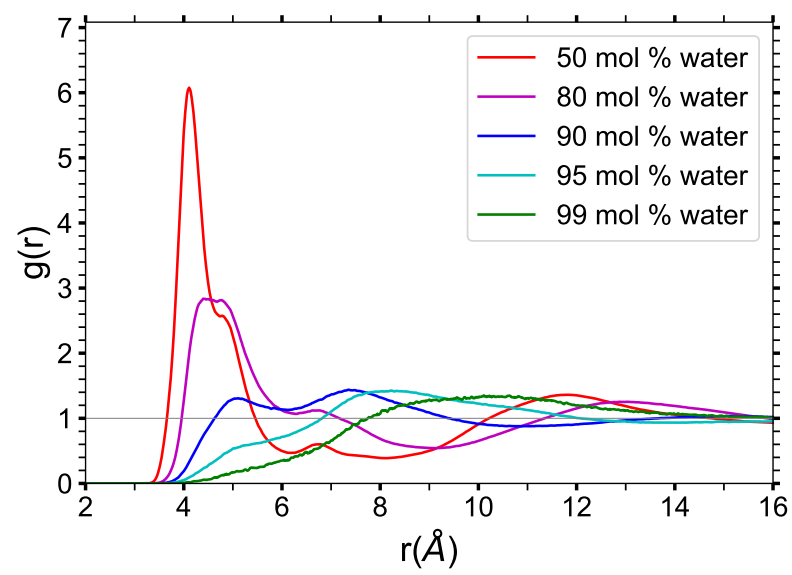

(b) $\mathrm{TBP}^{+}-\mathrm{TBP}^{+}$

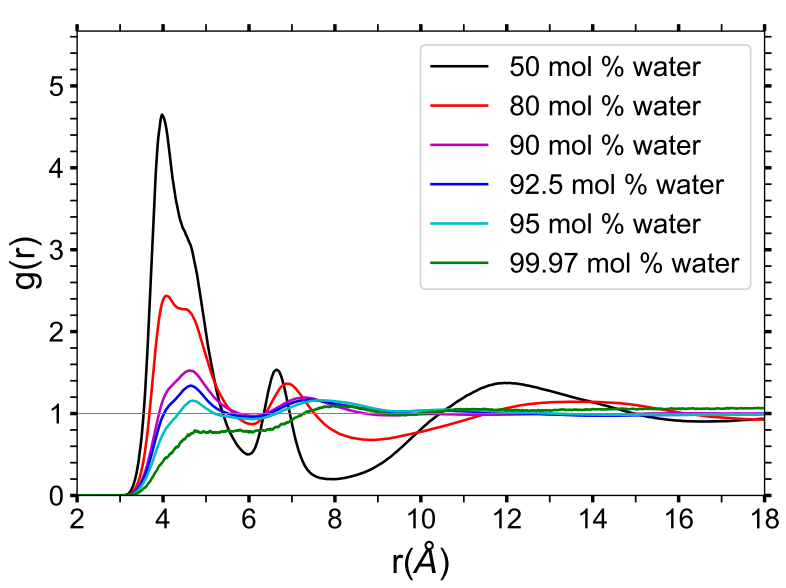

(c) $\mathrm{TBP}^{+}-\mathrm{OH}^{-}$

(d) $\mathrm{TBP}^{+}-\mathrm{H}_{2} \mathrm{O}$

Figure (7.11) Radial distribution functions of TBPH-water at $300 \mathrm{~K}$ over a range of water concentrations (Part 1 of 2$){ }^{58}$ 


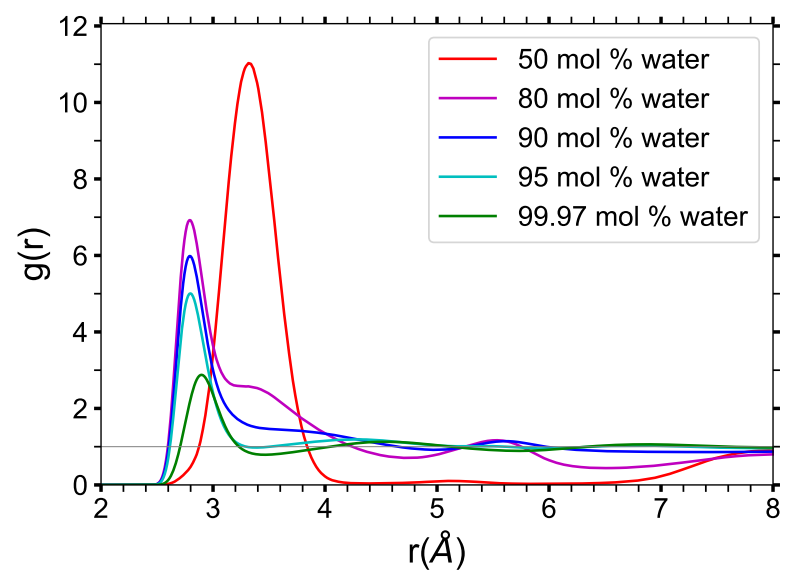

(a) $\mathrm{H}_{2} \mathrm{O}-\mathrm{H}_{2} \mathrm{O}$

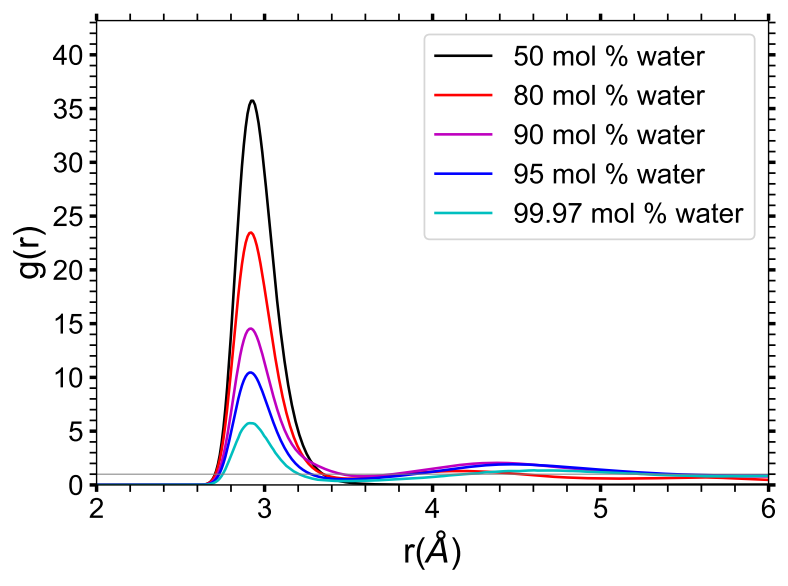

(b) $\mathrm{H}_{2} \mathrm{O}-\mathrm{OH}^{-}$

Figure (7.12) Radial distribution functions of TBPH-water at $300 \mathrm{~K}$ over a range of water concentrations (Part 2 of 2). ${ }^{58}$

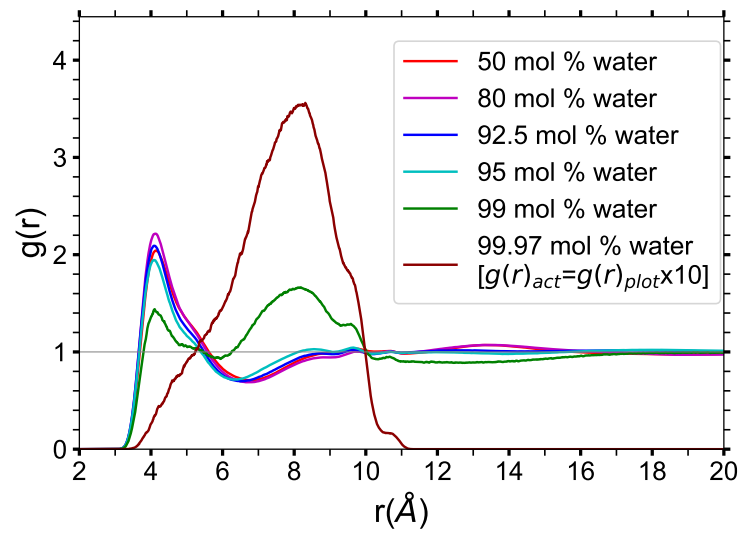

(a)

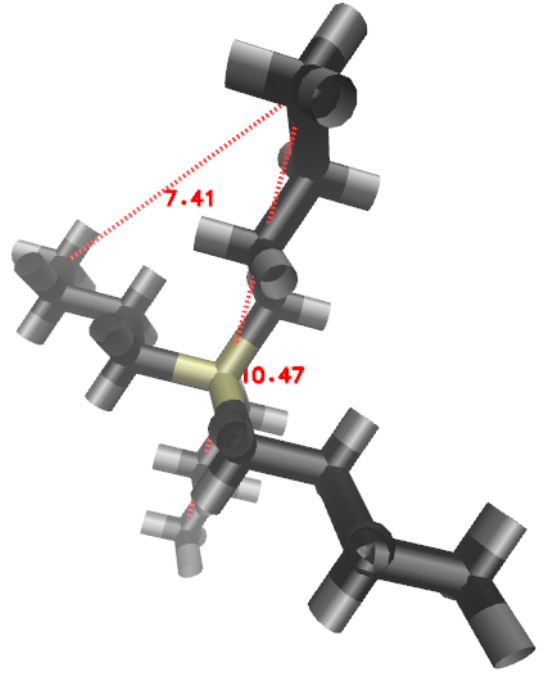

(b)

Figure (7.13) (a) Radial distribution functions of the end carbons on the TBP's butyl chains (CT4-CT4) for the TBPH-water solution at $300 \mathrm{~K}$ : Note that the $99.97 \mathrm{~mol} \%$ water data is an order of magnitude higher than the other data since it was scaled down by 1/10 to fit on the same plot; (b) Radial distances of the end carbons on the TBP's butyl chains (CT4's), within the same molecule. ${ }^{30,58}$ 


\subsection{Water Vein Formation in the TBPH-water Solutions}

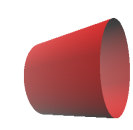

Hydroxide

$(\mathrm{OH})$ anion

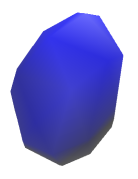

Water

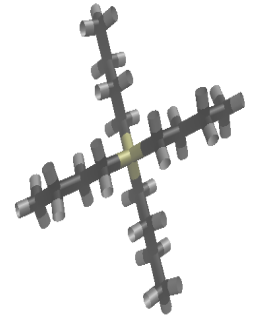

Tetrabutylphosphonium

$(\mathrm{TBP}+)$ cation

Figure (7.14) Depiction of molecules in the TBPH-water solution. ${ }^{58}$ The blue-colored water is represented using an isosurface (called quicksurf in $\mathrm{VMD}^{30}$ ), which uses a volumetric Gaussian density map of the water to produce the observable surface. The TBP and OH molecules are represented using dynamic bonds in VMD. ${ }^{30}$ TBP is colored tan, black and gray, for the phosphorus, carbon, and hydrogen atoms, respectively. The hydroxide is colored in red for both the oxygen and hydrogen atom. 


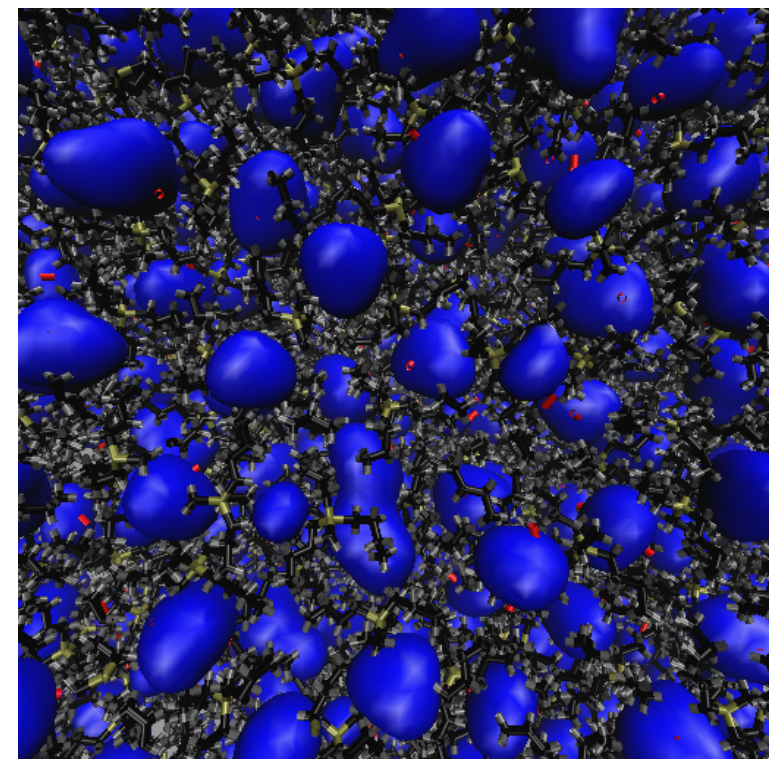

(a) $63.1 \mathrm{~mol} \%$ water

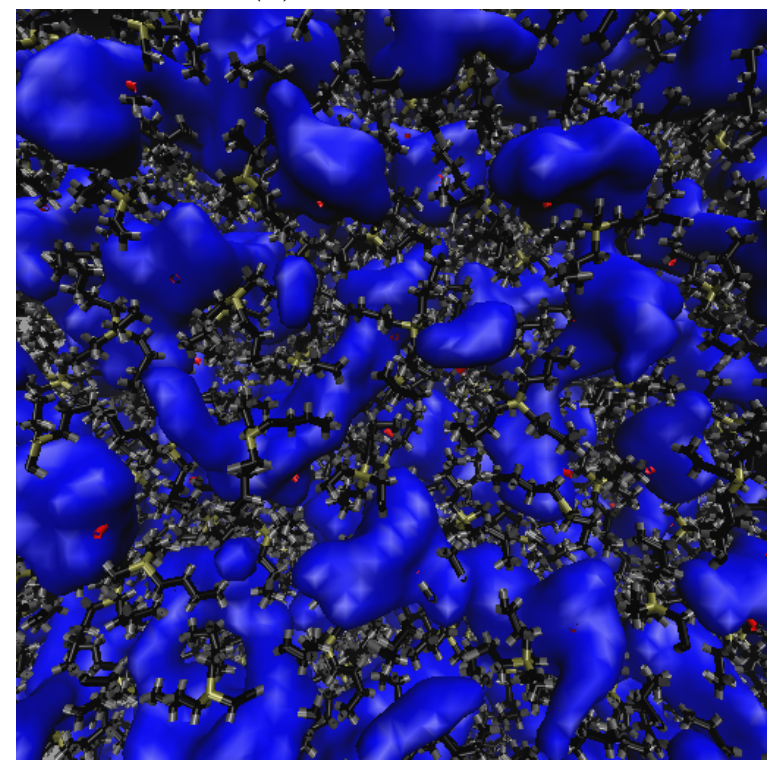

(c) $86.8 \mathrm{~mol} \%$ water

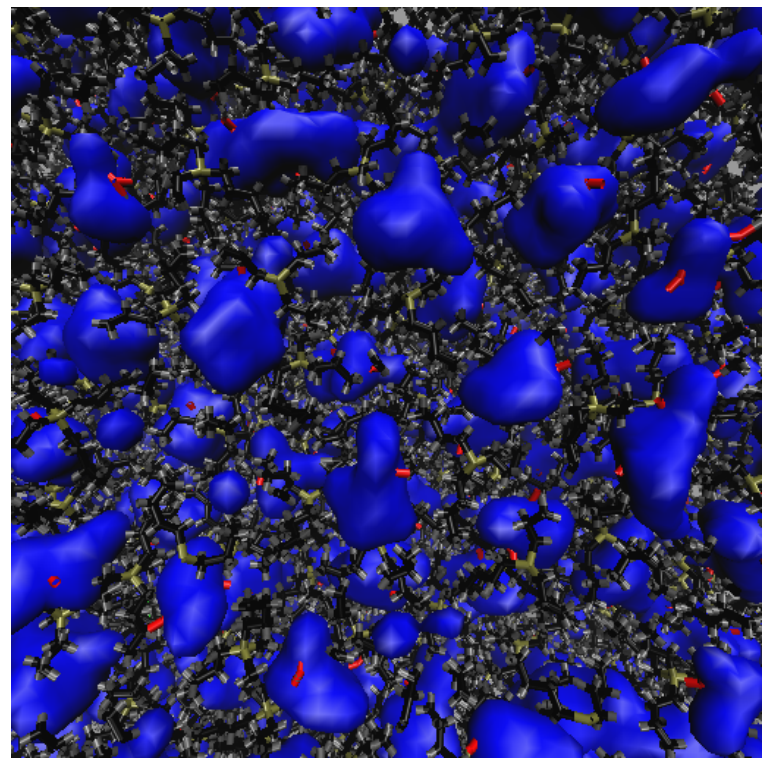

(b) $79.4 \mathrm{~mol} \%$ water

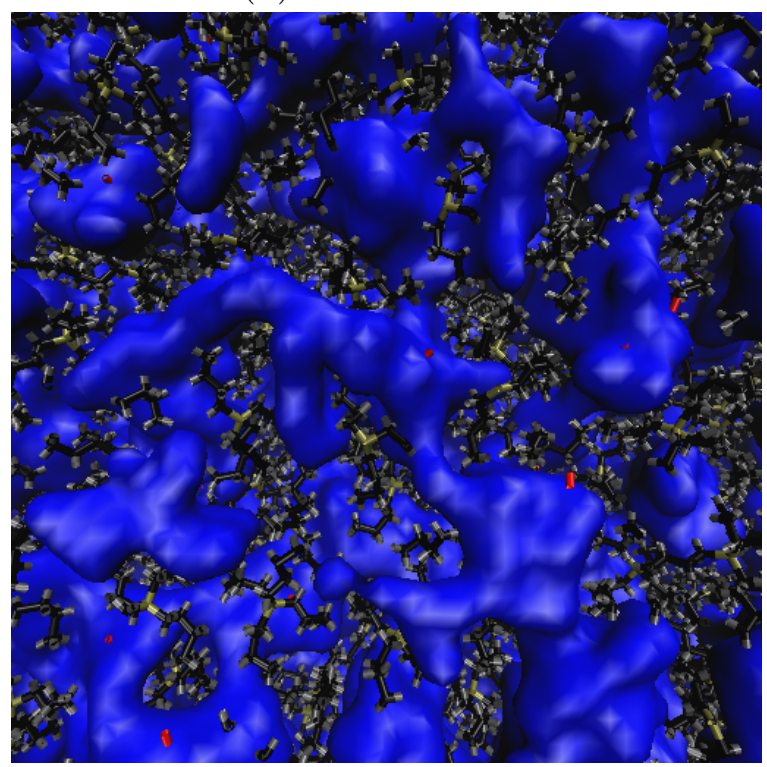

(d) $91.1 \mathrm{~mol} \%$ water

Figure (7.15) Water vein/channeling formation in TBPH-water at $320 \mathrm{~K}$ (Part 1 of 2). ${ }^{53,54,58}$ The blue-colored water is represented using an isosurface drawing method (called quicksurf in $\mathrm{VMD}^{30}$ ), which uses a volumetric Gaussian density map of the water to produce the observable surface. The TBP and $\mathrm{OH}$ molecules are represented using the dynamic bonds drawing method in VMD. ${ }^{30} \mathrm{TBP}$ is colored tan, black and gray, for the phosphorus, carbon, and hydrogen atoms, respectively. The hydroxide is colored in red for both the oxygen and hydrogen atom. 


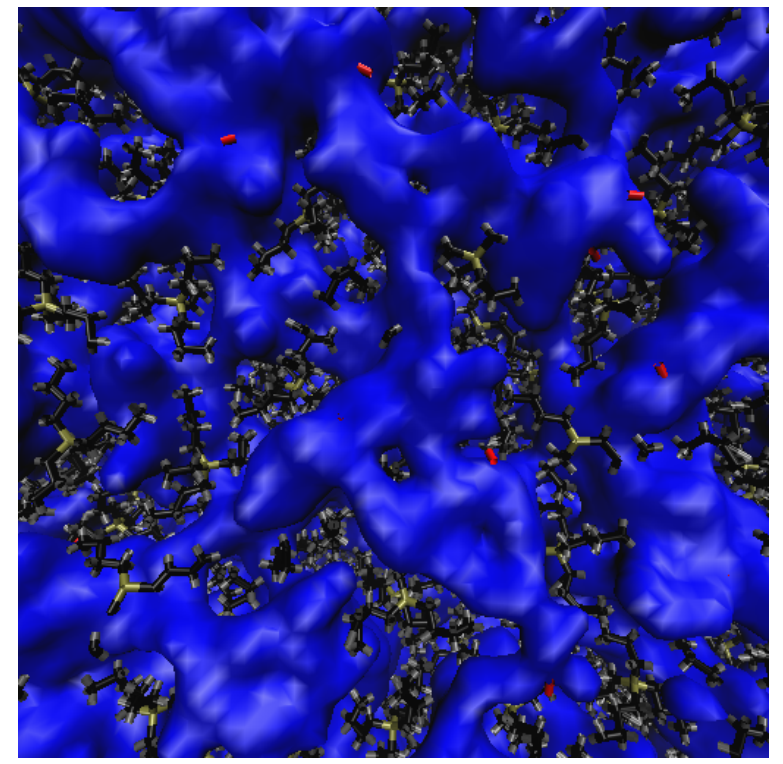

(a) $93.9 \mathrm{~mol} \%$ water

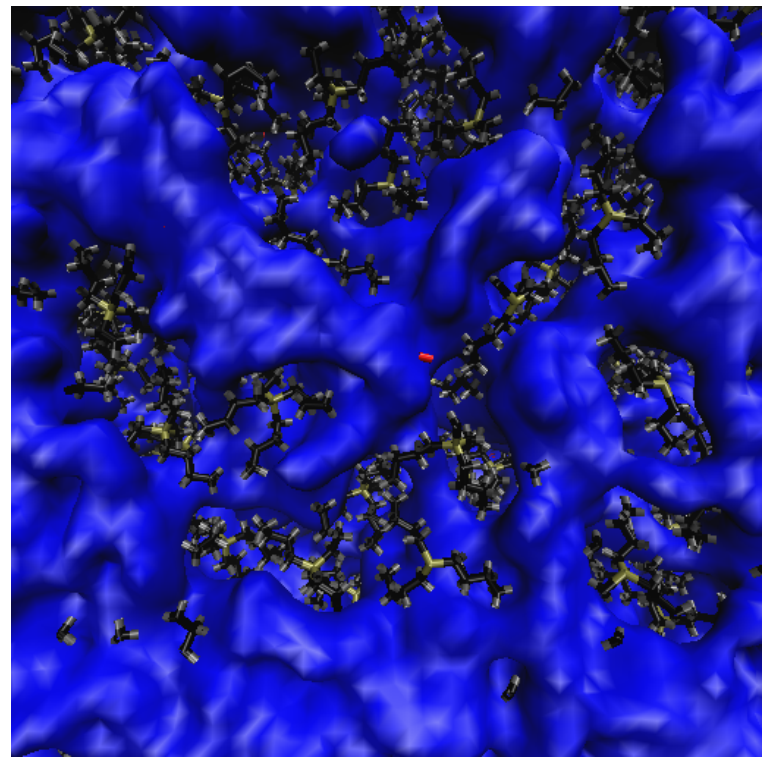

(b) $95.8 \mathrm{~mol} \%$ water

Figure (7.16) Water vein/channeling formation in TBPH-water at $320 \mathrm{~K}$ (Part 2 of 2). ${ }^{53,54,58}$ The blue-colored water is represented using an isosurface drawing method (called quicksurf in $\mathrm{VMD}^{30}$ ), which uses a volumetric Gaussian density map of the water to produce the observable surface. The TBP and $\mathrm{OH}$ molecules are represented using the dynamic bonds drawing method in VMD. ${ }^{30}$ TBP is colored tan, black and gray, for the phosphorus, carbon, and hydrogen atoms, respectively. The hydroxide is colored in red for both the oxygen and hydrogen atom. 


\subsection{Diffusion Properties: TBPH-Water Solutions (cont.)}

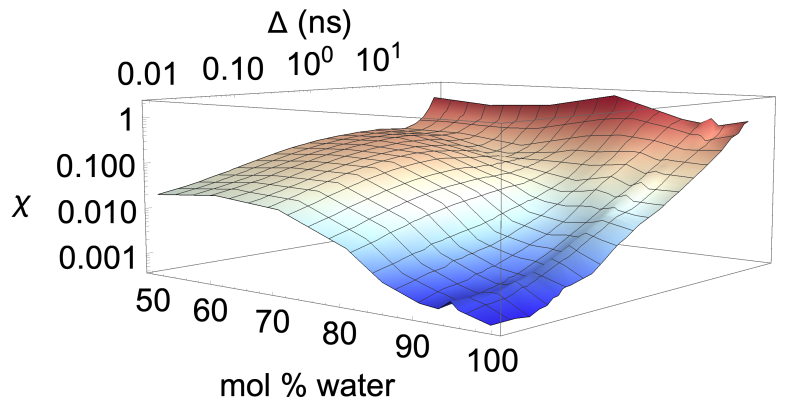

(a) $\mathrm{OH}^{-}$

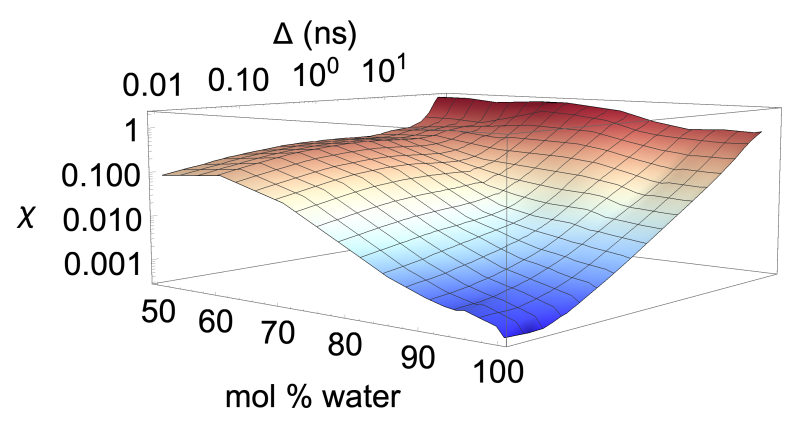

(b) Water

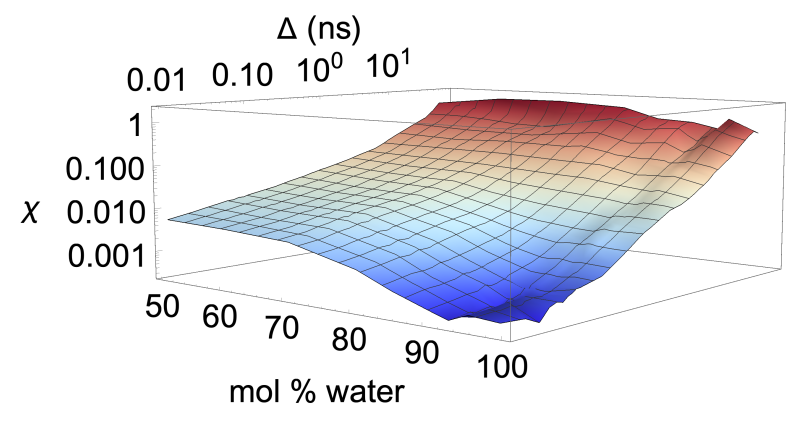

(c) $T B P^{+}$

Figure (7.17) Ergodicity breaking parameter $(\chi)$ in TBPH-water at $p=1 \mathrm{~atm}$ and $T=$ $300 \mathrm{~K}$ as a function of water concentration and lag time. ${ }^{23,58}$ 


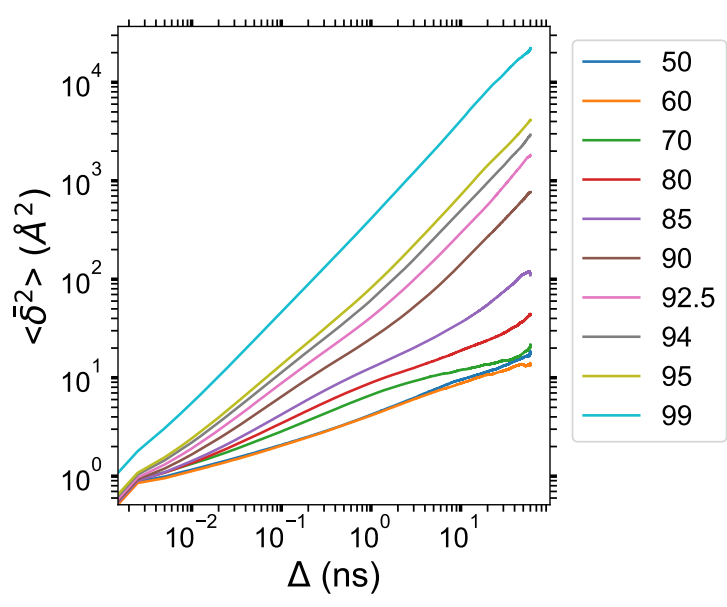

(a) $\mathrm{OH}^{-}$

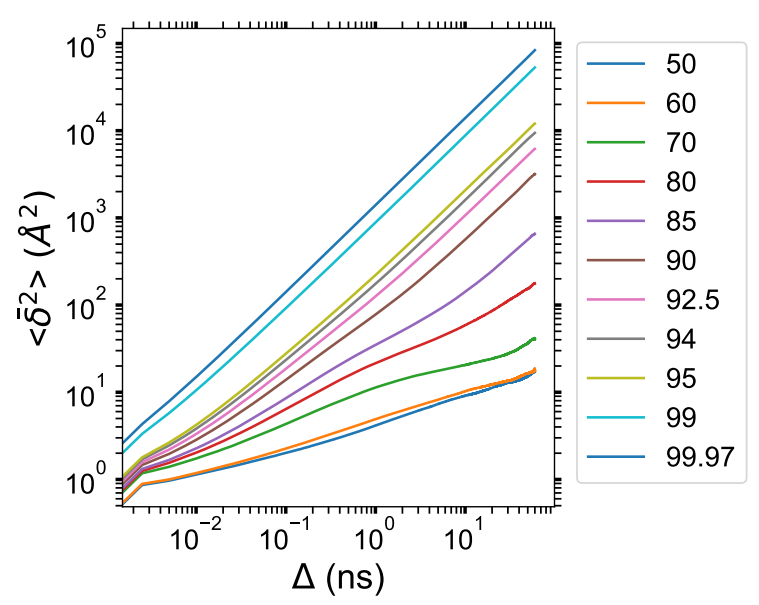

(b) Water

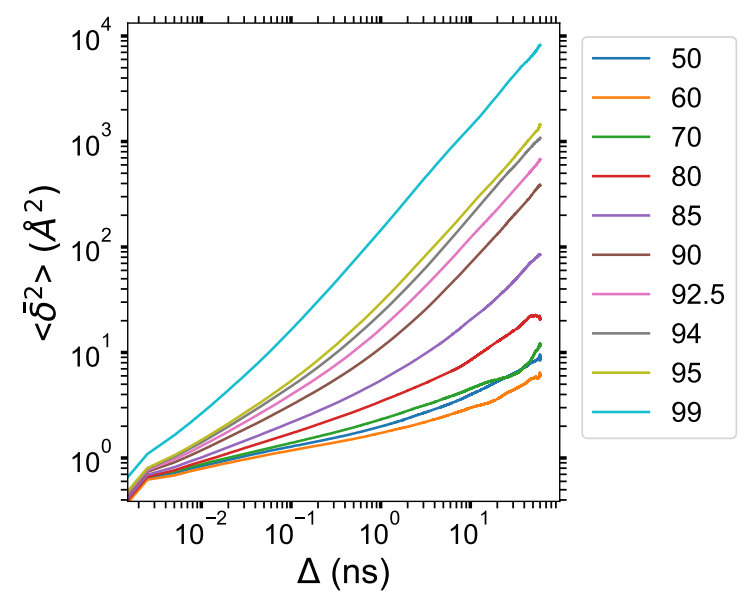

(c) $T B P^{+}$

Figure (7.18) Particle-averaged TAMSDs of the TBPH-water solution at $p=1 \mathrm{~atm}$ and $T=300 \mathrm{~K}$ as a function of water concentration. ${ }^{58}$ 


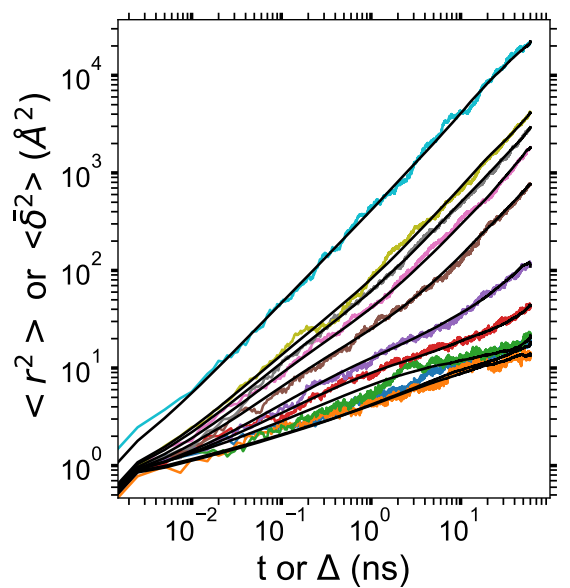

(a) $\log$ scale $O H^{-}$

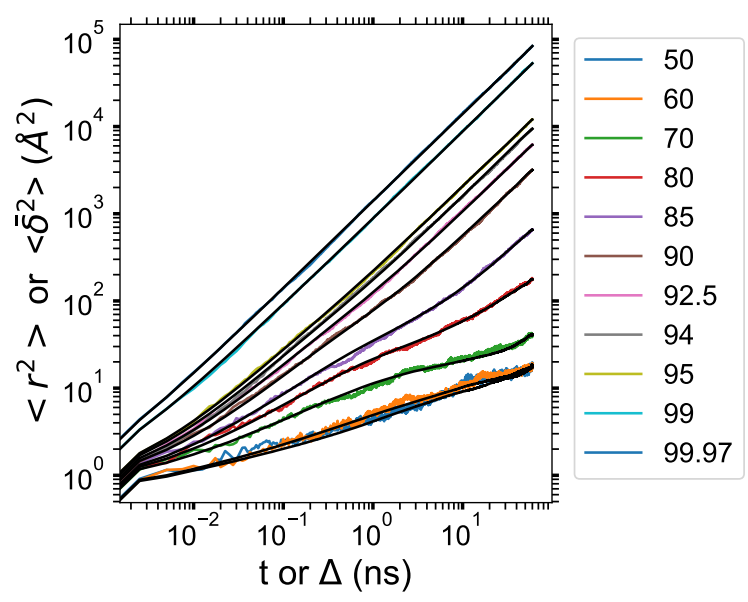

(c) Log scale Water

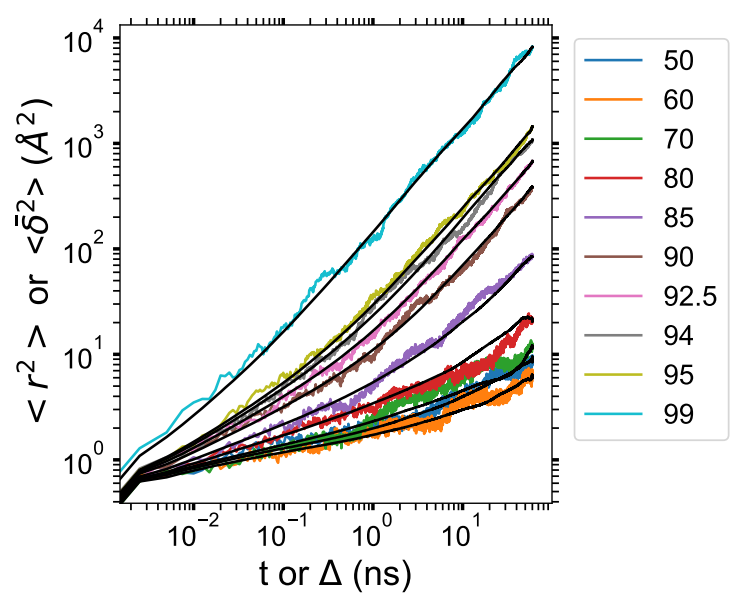

(e) $\log$ scale $T B P^{+}$

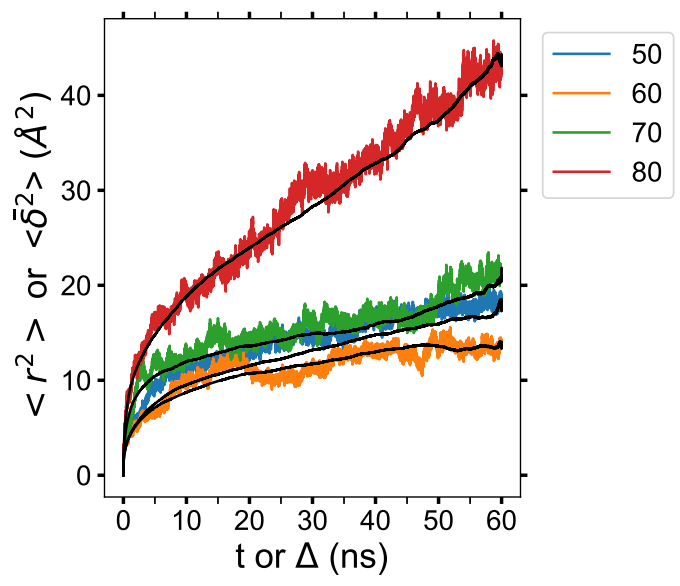

(b) Linear scale $\mathrm{OH}^{-}$

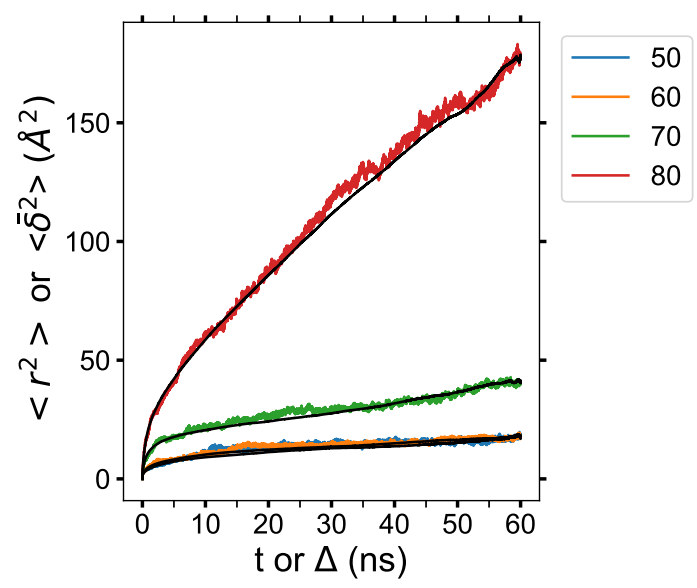

(d) Linear scale Water

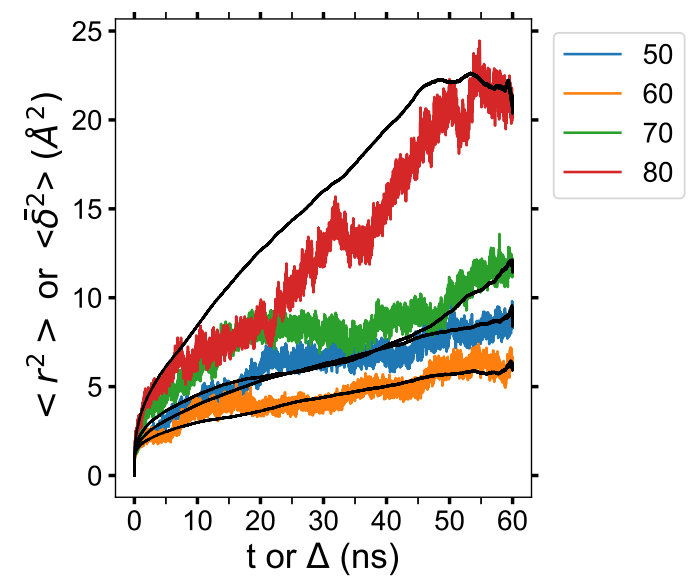

(f) Linear scale $T B P^{+}$

Figure (7.19) MSD vs. particle-averaged TAMSD of the water, TBP and OH in TBPHwater at $p=1 \mathrm{~atm}$ and $T=300 \mathrm{~K}$ as a function of water concentration. ${ }^{58}$ The colored lines are the MSDs, and the black lines are the particle-averaged TAMSDs. 
Table (7.10) TBPH anomalous diffusion coefficients of water, $\mathrm{TBP}^{+}$, and $\mathrm{OH}^{-}$

\begin{tabular}{|c|c|c|c|c|c|c|}
\hline \multirow[b]{3}{*}{ Mole $\%$} & \multicolumn{6}{|c|}{$K_{\alpha}\left(10^{-6} \mathrm{~cm}^{2} \mathrm{~s}^{-1}\right) / \alpha\left(1 / s^{\alpha}\right)$} \\
\hline & \multicolumn{2}{|c|}{$\mathrm{H}_{2} \mathrm{O}$} & \multicolumn{2}{|c|}{$\mathrm{TBP}^{+}$} & \multicolumn{2}{|c|}{$\mathrm{OH}^{-}$} \\
\hline & $K_{\alpha}$ & $\alpha$ & $K_{\alpha}$ & $\alpha$ & $K_{\alpha}$ & $\alpha$ \\
\hline 50.0 & 0.423 & 0.329 & 0.179 & 0.375 & 0.434 & 0.331 \\
\hline 60.0 & 0.496 & 0.304 & 0.151 & 0.320 & 0.440 & 0.289 \\
\hline 70.0 & 1.01 & 0.315 & 0.203 & 0.360 & 0.657 & 0.249 \\
\hline 80.0 & 1.82 & 0.536 & 0.297 & 0.496 & 0.786 & 0.390 \\
\hline 85.0 & 2.80 & 0.749 & 0.455 & 0.693 & 1.03 & 0.588 \\
\hline 90.0 & 7.17 & 0.917 & 1.01 & 0.872 & 2.16 & 0.855 \\
\hline 92.5 & 12.2 & 0.954 & 1.62 & 0.894 & 3.65 & 0.937 \\
\hline 94.0 & 17.2 & 0.977 & 2.34 & 0.935 & 5.97 & 0.934 \\
\hline 95.0 & 22.1 & 0.975 & 2.93 & 0.932 & 8.22 & 0.949 \\
\hline 99.0 & 87.3 & 1.00 & 14.7 & 0.978 & 43.5 & 0.969 \\
\hline $99.97^{a}$ & 139 & 0.999 & -- & -- & -- & -- \\
\hline
\end{tabular}

${ }^{a}$ Infinite-dilution simulation for TBPH

Data for $p=1 \mathrm{~atm}$ and $T=300 \mathrm{~K}^{58}$ 


\subsection{Cellulose Dissolution in TBPCl-water (cont.)}

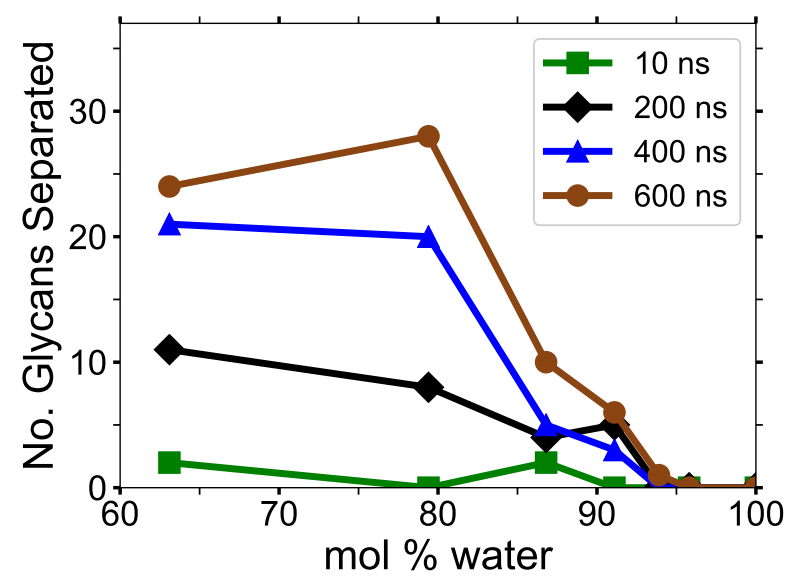

Figure (7.20) TBPCl-water small cellulose bundles dissolution measured by the number of dissolved glycans at $360 \mathrm{~K}^{22}$

Table (7.11) Small cellulose bundle dissolution rates ${ }^{22}$

\begin{tabular}{cc}
$\begin{array}{c}\text { Mol \% } \\
\text { Water }\end{array}$ & $\begin{array}{c}\text { Cellulose dissolution rate } \\
(\text { wt } \% / \mathrm{ns})\end{array}$ \\
\hline 63.1 & $1.443 \times 10^{-3}$ \\
79.4 & $1.505 \times 10^{-3}$ \\
86.8 & $0.501 \times 10^{-3}$ \\
91.1 & $0.326 \times 10^{-3}$ \\
93.9 & $0.033 \times 10^{-3}$ \\
95.8 & $0.000 \times 10^{-3}$ \\
100 & $0.000 \times 10^{-3}$ \\
\hline
\end{tabular}




\subsection{Cellulose Strand Separation in TBPCl-water (cont.)}

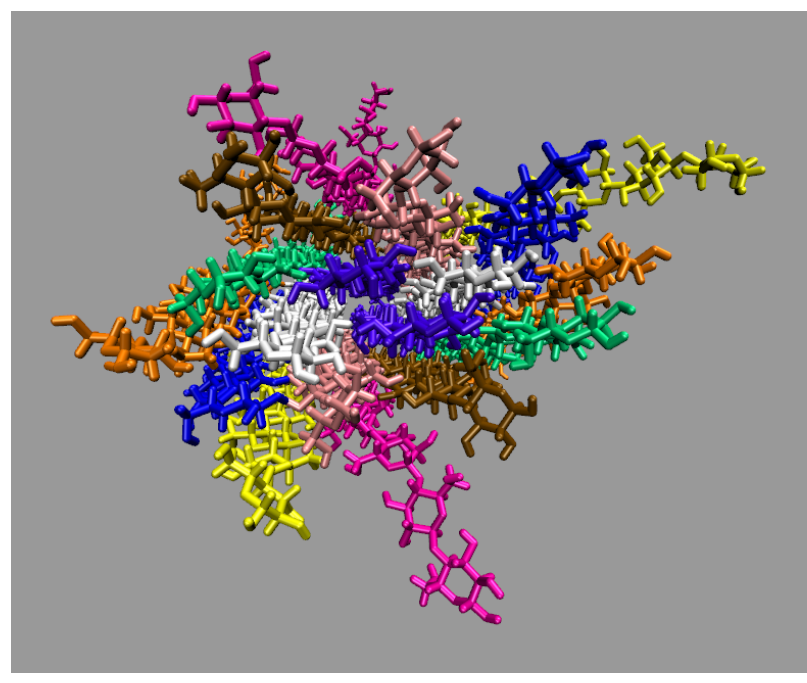

(a) $63.1 \mathrm{~mol} \%$ water (cellulose only)

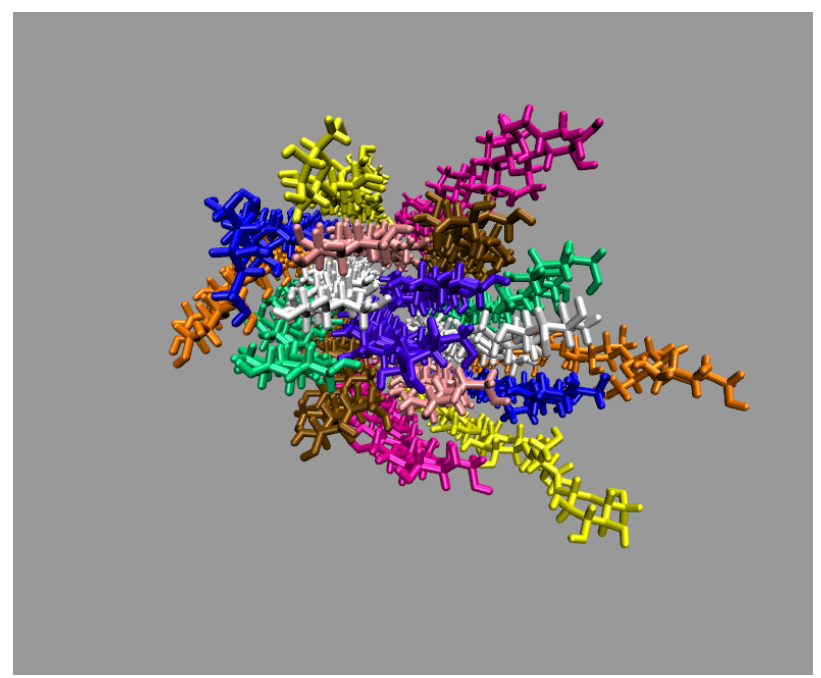

(c) $86.8 \mathrm{~mol} \%$ water (cellulose only)

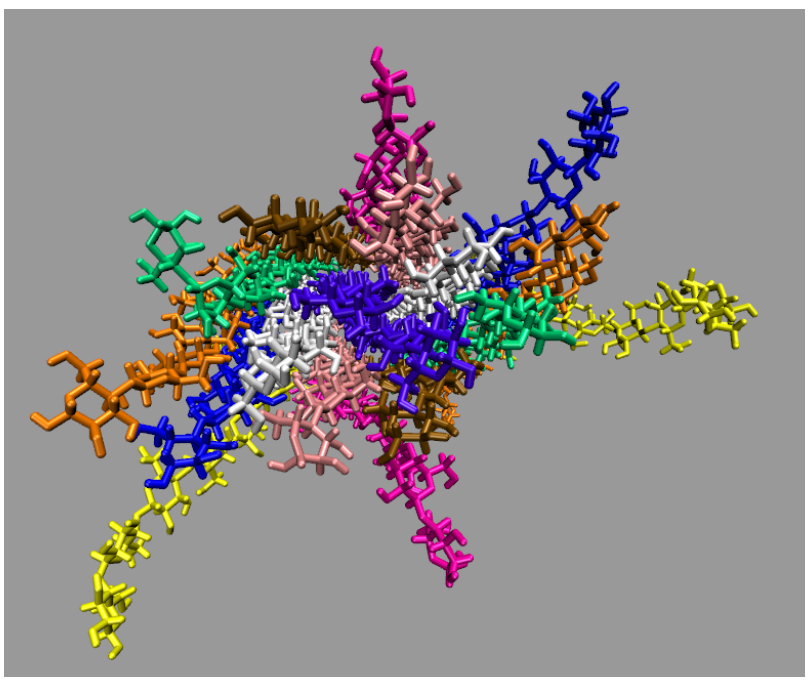

(b) $79.4 \mathrm{~mol} \%$ water (cellulose only)

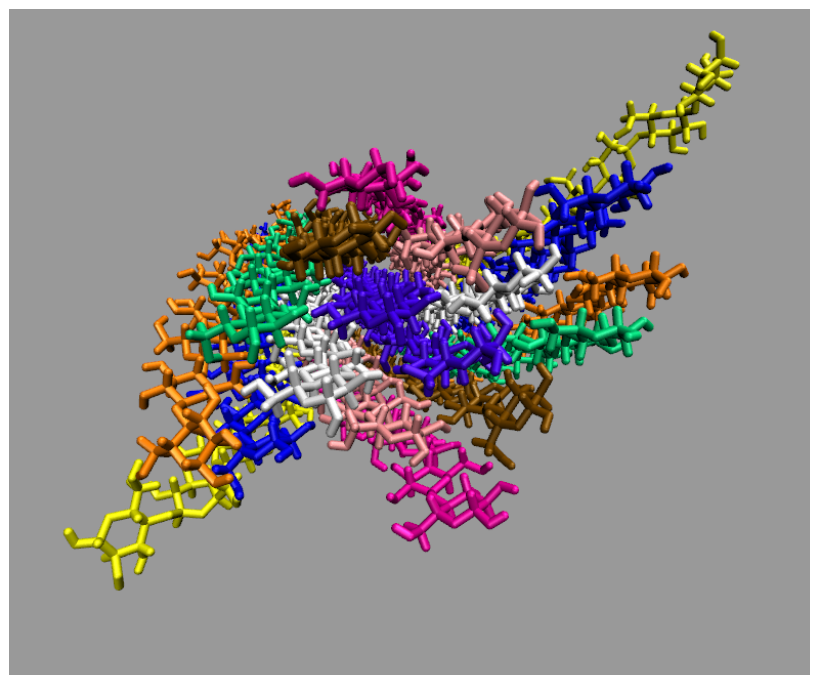

(d) $91.1 \mathrm{~mol} \%$ water (cellulose only)

Figure (7.21) End view images of the cellulose bundle dissolution at $600 \mathrm{~ns}$, showing only the cellulose bundle (Part 1 of 2 ). ${ }^{22}$ 


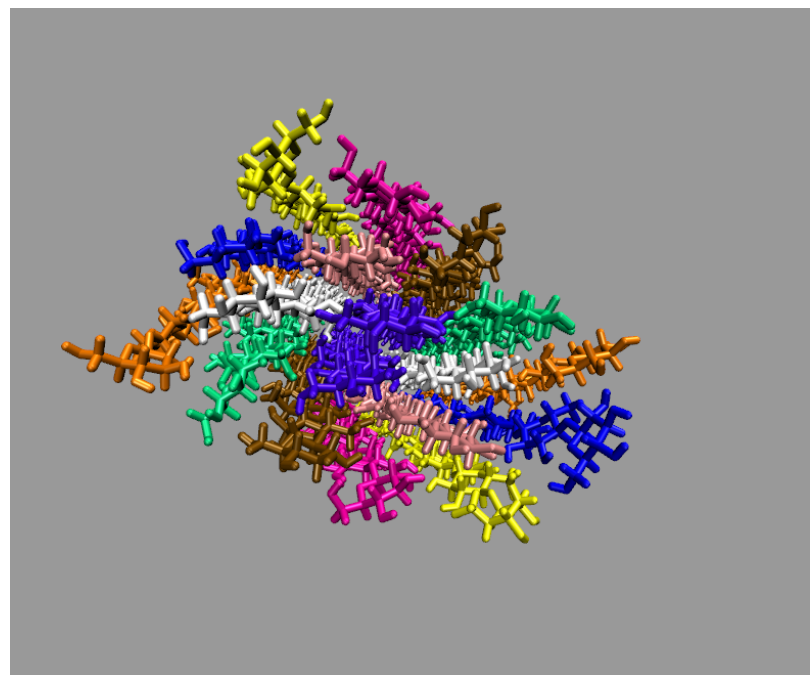

(a) $93.9 \mathrm{~mol} \%$ water (cellulose only)

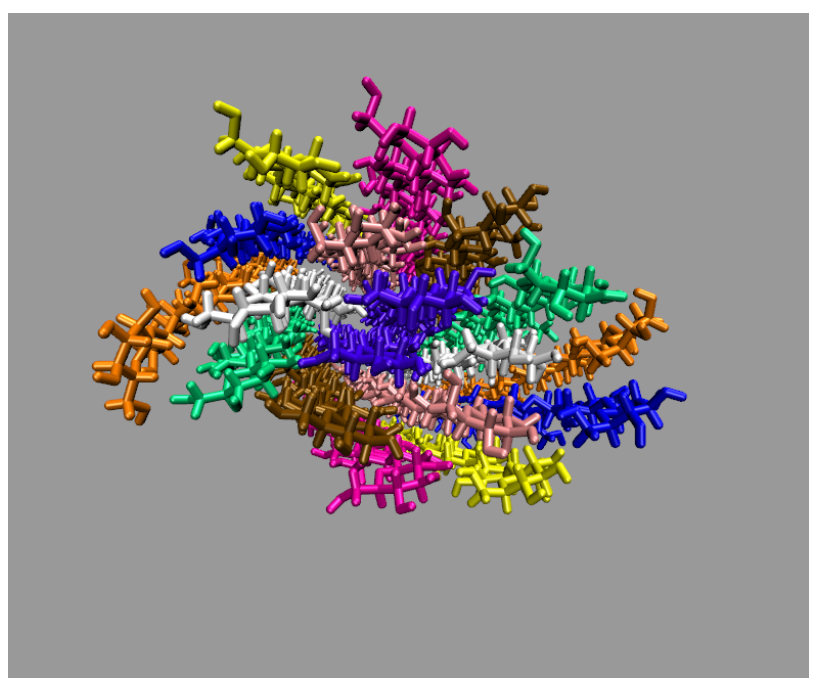

(b) $95.8 \mathrm{~mol} \%$ water (cellulose only)

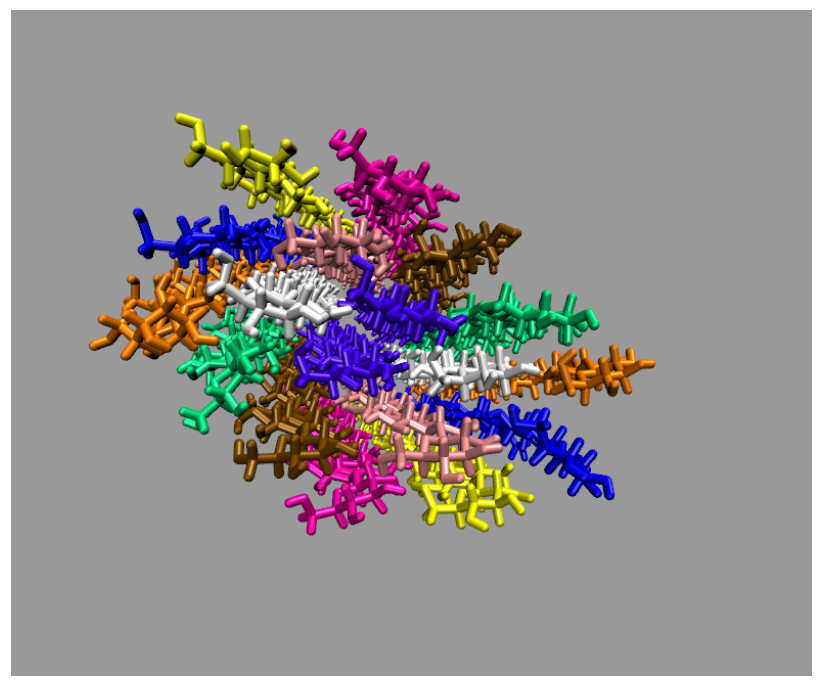

(c) $100 \mathrm{~mol} \%$ water (cellulose only)

Figure (7.22) End view images of the cellulose bundle dissolution at $600 \mathrm{~ns}$, showing only the cellulose bundle (Part 2 of 2). ${ }^{22}$ 


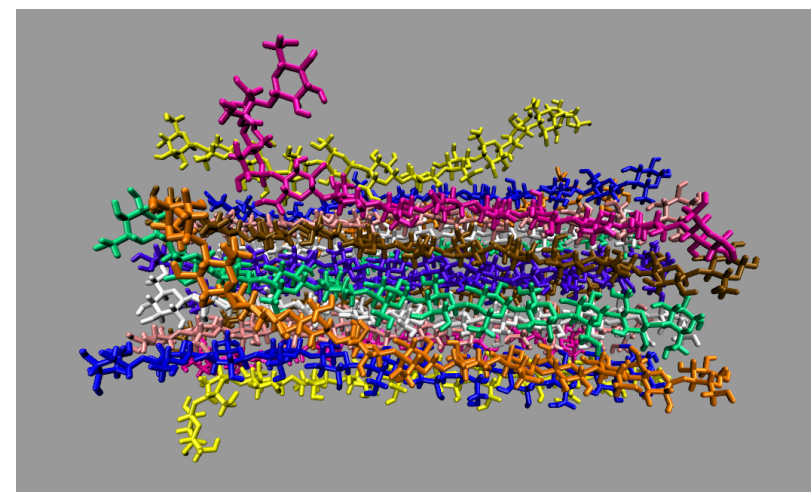

(a) $63.1 \mathrm{~mol} \%$ water (cellulose only)

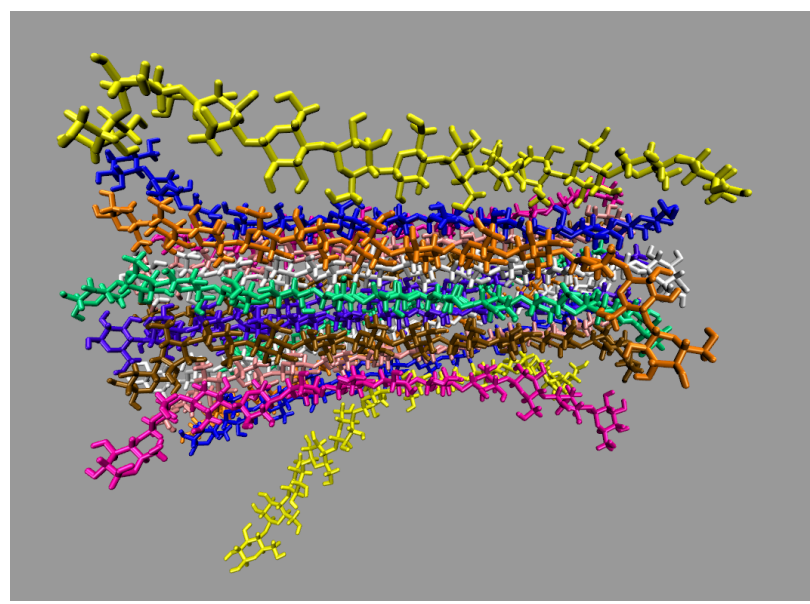

(c) $79.4 \mathrm{~mol} \%$ water (cellulose only)

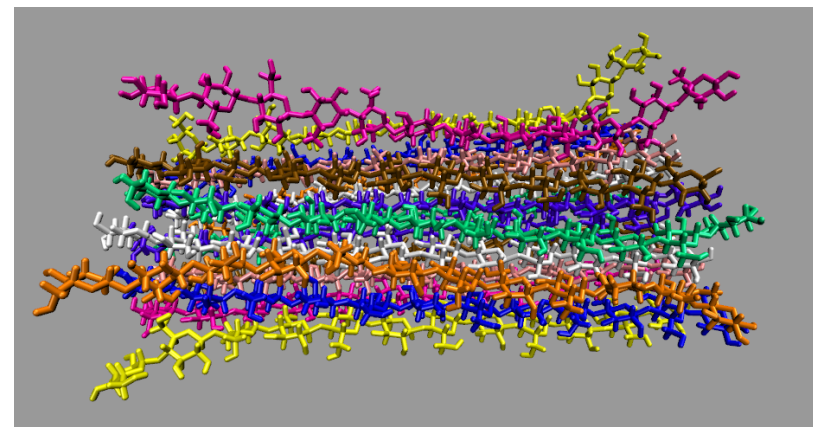

(e) $86.8 \mathrm{~mol} \%$ water (cellulose only)

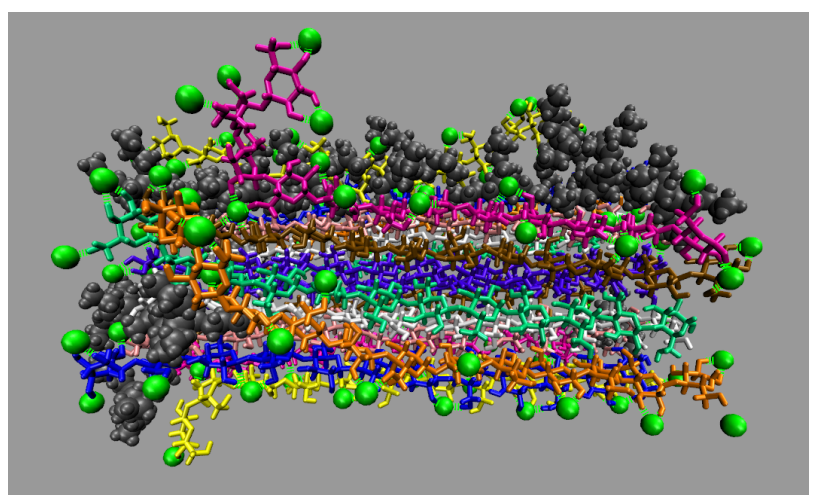

(b) $63.1 \mathrm{~mol} \%$ water (with TBP, Cl, and water)

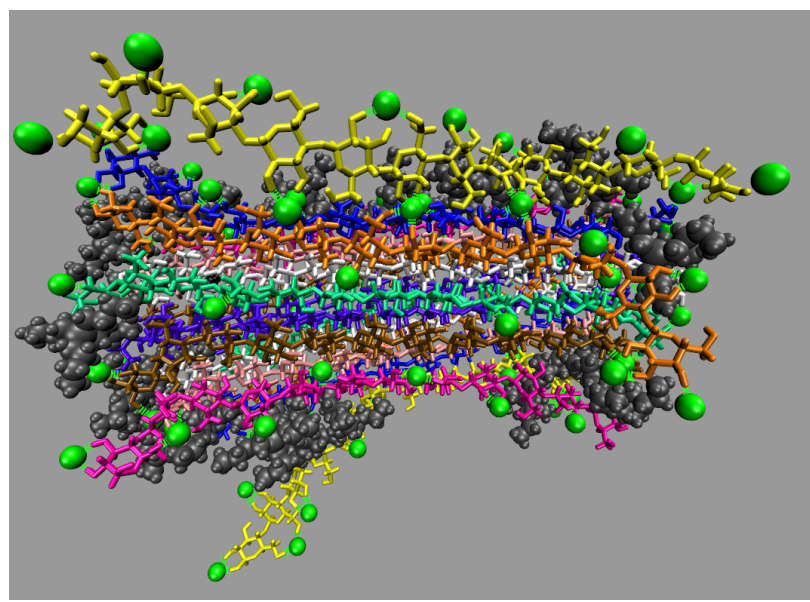

(d) $79.4 \mathrm{~mol} \%$ water (with TBP, Cl, and water)

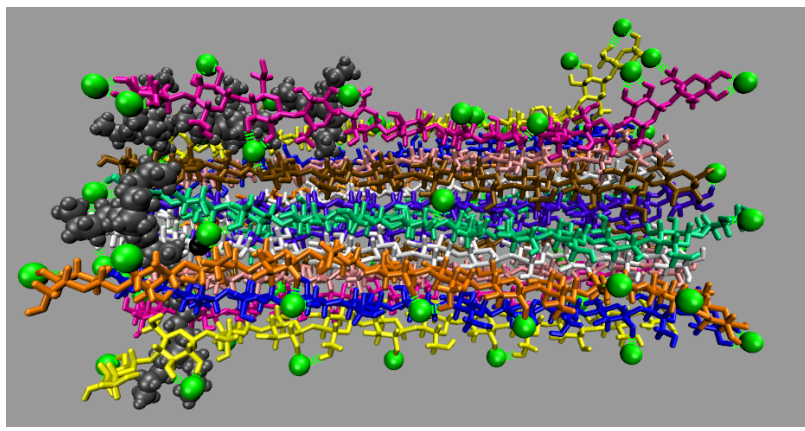

(f) $86.8 \mathrm{~mol} \%$ water (with TBP, Cl, and water)

Figure (7.23) Side view images of the cellulose bundle dissolution at $600 \mathrm{~ns}$, showing all the nearby TBP, $\mathrm{Cl}$, and water molecules as described earlier (Part 1 of 2 ). ${ }^{22}$ 


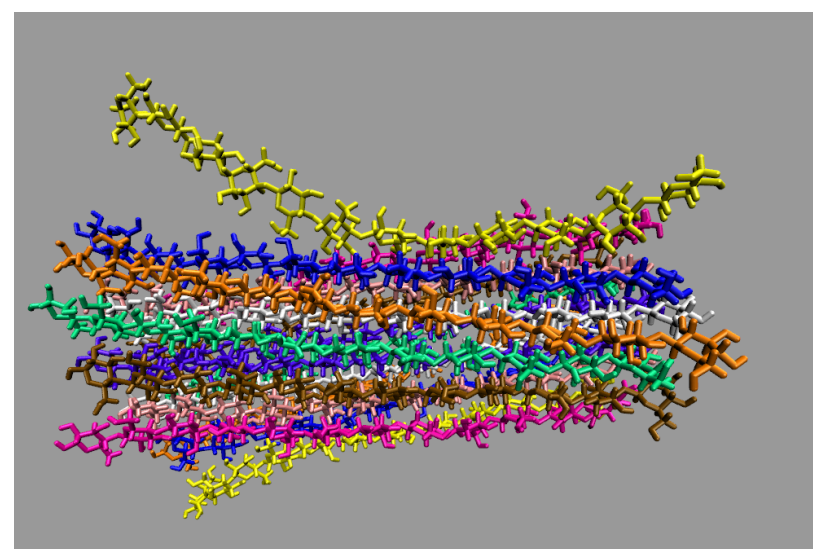

(a) $91.1 \mathrm{~mol} \%$ water (cellulose only)

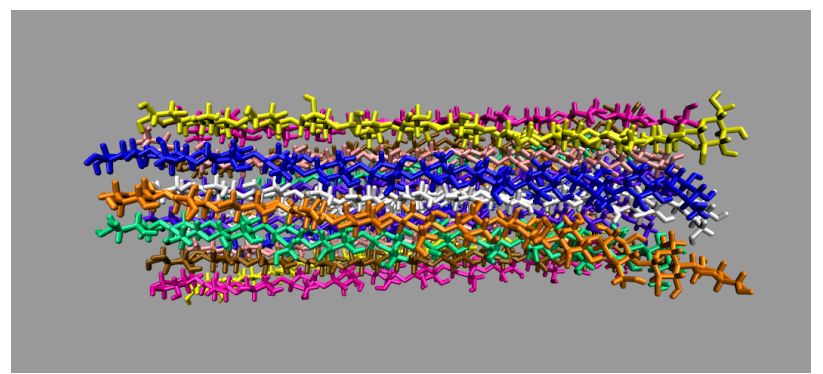

(c) $93.9 \mathrm{~mol} \%$ water (cellulose only)

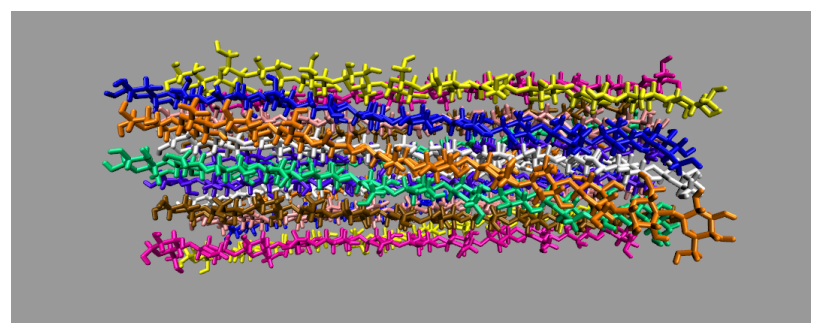

(e) $95.8 \mathrm{~mol} \%$ water (cellulose only)

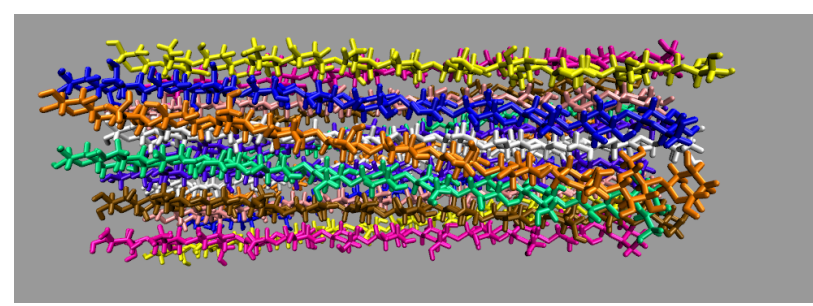

(g) $100 \mathrm{~mol} \%$ water (cellulose only)

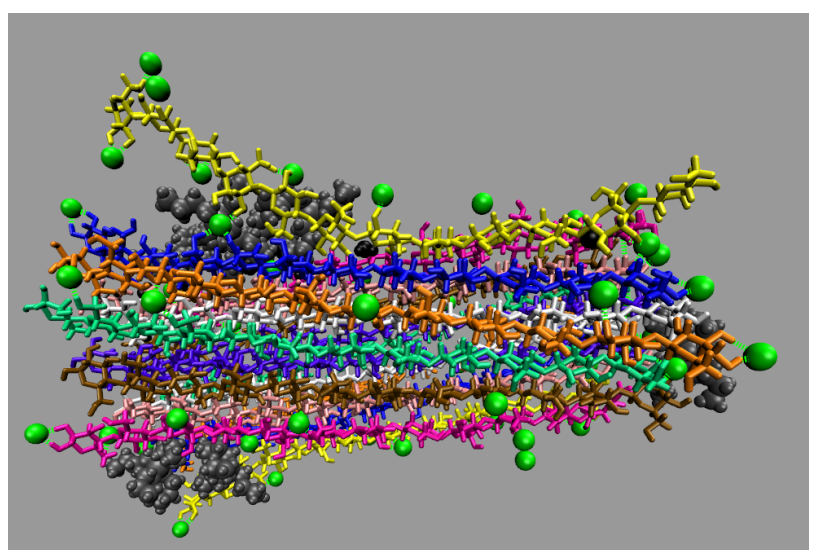

(b) $91.1 \mathrm{~mol} \%$ water (with TBP, Cl, and water)

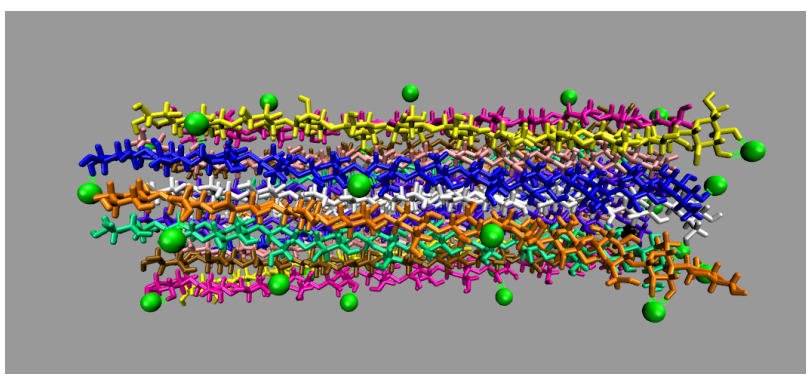

(d) $93.9 \mathrm{~mol} \%$ water (with $\mathrm{TBP}, \mathrm{Cl}$, and water)

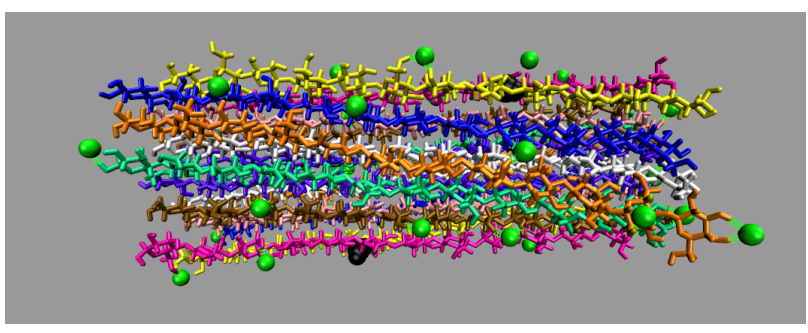

(f) $95.8 \mathrm{~mol} \%$ water (with TBP, Cl, and water)

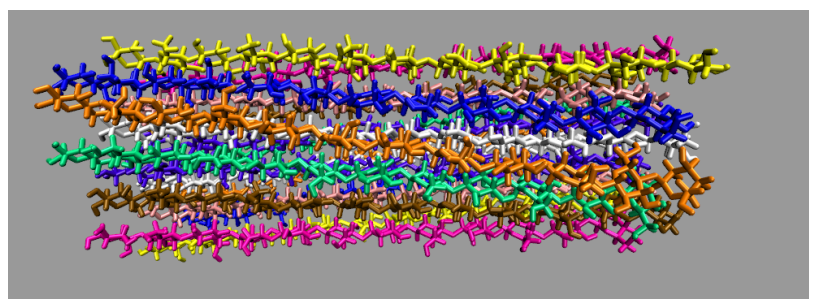

(h) $100 \mathrm{~mol} \%$ water (with TBP, Cl, and water)

Figure (7.24) Side view images of the cellulose bundle dissolution at $600 \mathrm{~ns}$, showing all the nearby TBP, Cl, and water molecules (Part 2 of 2 ). ${ }^{22}$ 


\subsection{Hydrogen Bonding Lifetimes in the TBPCl-water and Cellu- lose Solutions (cont.)}

Table (7.12) TBPCl hydrogen bonding lifetimes (ps), Part 3 of 5

\begin{tabular}{|c|c|c|c|c|c|c|c|}
\hline \multirow[b]{2}{*}{$\mathrm{A} \cdots \mathrm{H}-\mathrm{D}^{a}$} & \multicolumn{7}{|c|}{ mol \% water } \\
\hline & 63.1 & 79.4 & 86.8 & 91.1 & 93.9 & 95.8 & 100 \\
\hline $\mathrm{O} 2 \cdots \mathrm{H} 1-\mathrm{C} 1$ (10 ns) & 16.4 & 103 & 105 & 25.8 & 18.4 & 8.62 & 3.51 \\
\hline $\mathrm{O} 2 \cdots \mathrm{H} 1-\mathrm{C} 1$ (400 ns) & 13.9 & 33.1 & 171 & 14.0 & 12.7 & 146 & 24.7 \\
\hline $\mathrm{O} 2 \cdots \mathrm{H} 2-\mathrm{C} 2(10 \mathrm{~ns})$ & 0.290 & 1.03 & 0.196 & 0.241 & 0.247 & 0.244 & 0.248 \\
\hline $\mathrm{O} 2 \cdots \mathrm{H} 2-\mathrm{C} 2(400 \mathrm{~ns})$ & 0.907 & 0.222 & 0.433 & 0.339 & 0.554 & 0.322 & 0.247 \\
\hline O2 $\cdots$ H3-C3 (10 ns) & 1.86 & 2.37 & 1.88 & 2.03 & 1.98 & 2.04 & 2.44 \\
\hline $\mathrm{O} 2 \cdots \mathrm{H} 3-\mathrm{C} 3(400 \mathrm{~ns})$ & 1.10 & 2.14 & 1.68 & 1.80 & 1.73 & 2.16 & 1.67 \\
\hline O2*H4-C4 (10 ns) & 3,661 & 3,077 & 3,304 & 2,230 & 2,039 & 4,170 & 414 \\
\hline $\mathrm{O} 2 \cdots \mathrm{H} 4-\mathrm{C} 4(400 \mathrm{~ns})$ & 49.8 & 386 & 2,598 & 415 & 419 & 492 & 2,193 \\
\hline O2*H5-C5 (10 ns) & 0.679 & 0.359 & 0.166 & 0.242 & 0.301 & 0.183 & 0.237 \\
\hline $\mathrm{O} 2 \cdots \mathrm{H} 5-\mathrm{C} 5(400 \mathrm{~ns})$ & 1.22 & 0.766 & 0.176 & 0.234 & 0.682 & 0.215 & 0.324 \\
\hline $\mathrm{O} 2 \cdots \mathrm{H} 6^{\prime} \mathrm{s}-\mathrm{C} 6^{b}(10 \mathrm{~ns})$ & 0.589 & 0.588 & 0.505 & 0.545 & 0.480 & 0.381 & 0.410 \\
\hline $\mathrm{O} 2 \cdots \mathrm{H} 6{ }^{\prime} \mathrm{s}-\mathrm{C} 6^{b}$ (400 ns) & 0.541 & 0.680 & 0.619 & 0.600 & 0.588 & 0.530 & 0.465 \\
\hline 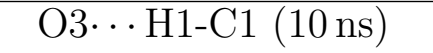 & 1,703 & 14.9 & 22.6 & 4.05 & 16.2 & 12.7 & 11.8 \\
\hline O3 $\cdots$ H1-C1 (400 ns) & 213 & 39.6 & 33.8 & 193 & 148 & 24.5 & 4.27 \\
\hline $\mathrm{O} 3 \cdots \mathrm{H} 2-\mathrm{C} 2(10 \mathrm{~ns})$ & 237 & 36.2 & 38.0 & 34.5 & 40.6 & 46.0 & 41.3 \\
\hline $\mathrm{O} 3 \cdots \mathrm{H} 2-\mathrm{C} 2(400 \mathrm{~ns})$ & 41.1 & 35.7 & 23.4 & 32.2 & 29.5 & 32.3 & 33.3 \\
\hline 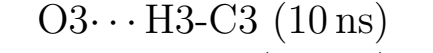 & 0.948 & 0.701 & 0.662 & 0.934 & 1.01 & 0.771 & 0.843 \\
\hline O3 $\cdots$ H3-C3(400 ns) & 0.628 & 0.836 & 0.978 & 0.807 & 0.953 & 0.726 & 0.657 \\
\hline O3*H4-C4 (10 ns) & 1.23 & 0.405 & 0.234 & 0.402 & 0.977 & 0.240 & 0.316 \\
\hline O3 $\cdots \mathrm{H} 4-\mathrm{C} 4$ (400 ns) & 0.262 & 0.571 & 1.16 & 1.20 & 0.473 & 0.166 & 0.292 \\
\hline O3*H5-C5 (10 ns) & 278 & 223 & 2,812 & 2,337 & 2,924 & 34.4 & 9.23 \\
\hline O3 $\cdots \mathrm{H} 5-\mathrm{C} 5(400 \mathrm{~ns})$ & 4364 & 321 & 1,618 & 401 & $>5210$ & 37.5 & 1,455 \\
\hline O3 $\cdots \mathrm{H} 6{ }^{\prime} \mathrm{s}-\mathrm{C} 6^{b}(10 \mathrm{~ns})$ & 0.119 & 0.144 & 0.134 & 0.113 & 0.187 & 0.107 & 0.114 \\
\hline $\mathrm{O} 3 \cdots \mathrm{H} 6^{\prime} \mathrm{s}-\mathrm{C} 6^{b}$ (400 ns) & 0.187 & 0.216 & 0.232 & 0.193 & 0.196 & 0.160 & 0.140 \\
\hline
\end{tabular}

${ }^{a} \mathrm{~A} \cdots \mathrm{H}-\mathrm{D}=$ hydrogen Acceptor atom $\cdots$ Hydrogen atom-hydrogen Donor atom. ${ }^{22}$ Note: (H-D) share a covalent bond. Data was started at 10.2 and $400.2 \mathrm{~ns}$.

${ }^{b}$ Averaged data

${ }^{c} \mathrm{NA}$ means no hydrogen bonds found at the start of these calculations.

${ }^{d}$ The H's are the HP and $\mathrm{HC} 2, \mathrm{HC} 3$, and $\mathrm{HC} 4$ atoms in the TBP molecule.

${ }^{f}$ MDAnalysis H-bond lifetimes do not work with $>100,000$ atoms in the analysis. For lifetimes without auto-correlation values of zero (i.e., values with $>$ symbol), the final auto-correlation values are $<0.05$ unless otherwise noted: ${ }^{g}<0.1 ;^{h}<0.15$. 
Table (7.13) TBPCl hydrogen bonding lifetimes (ps), Part 4 of 5

\begin{tabular}{|c|c|c|c|c|c|c|c|}
\hline \multirow[b]{2}{*}{$\mathrm{A} \cdots \mathrm{H}-\mathrm{D}^{a}$} & \multicolumn{7}{|c|}{ mol $\%$ water } \\
\hline & 63.1 & 79.4 & 86.8 & 91.1 & 93.9 & 95.8 & 100 \\
\hline O4* H1-C1 (10 ns) & $\mathrm{NA}^{c}$ & $\mathrm{NA}^{c}$ & $\mathrm{NA}^{c}$ & $\mathrm{NA}^{c}$ & $\mathrm{NA}^{c}$ & $\mathrm{NA}^{c}$ & $\mathrm{NA}^{c}$ \\
\hline O4 ․ H1-C1 (400 ns) & $\mathrm{NA}^{c}$ & 1.35 & $\mathrm{NA}^{c}$ & 0.137 & & $\mathrm{NA}^{c}$ & $\mathrm{NA}^{c}$ \\
\hline $\mathrm{O} 4 \cdots \mathrm{H} 2-\mathrm{C} 2(10 \mathrm{~ns})$ & $>50,596$ & $>48,068$ & 20,473 & 11,651 & 10,386 & 29,122 & 4,834 \\
\hline $\mathrm{O} 4 \cdots \mathrm{H} 2-\mathrm{C} 2(400 \mathrm{~ns})$ & $>57,152^{h}$ & $>29,957^{g}$ & $>13,465$ & $>13,689$ & 15,605 & $>24,677$ & 5,030 \\
\hline O4 ․ H3-C3 (10 ns) & $\mathrm{NA}^{c}$ & 0.609 & $\mathrm{NA}^{c}$ & $\mathrm{NA}^{c}$ & $\mathrm{NA}^{c}$ & $\mathrm{NA}^{c}$ & $\mathrm{NA}^{c}$ \\
\hline O4 ‥ H3-C3 (400 ns) & 1,154 & 3,140 & 8.09 & 6.13 & 7.71 & $\mathrm{NA}^{c}$ & 1.45 \\
\hline $\mathrm{O} 4 \cdots \mathrm{H} 4-\mathrm{C} 4(10 \mathrm{~ns})$ & $\mathrm{NA}^{c}$ & 0.126 & 0.243 & $\mathrm{NA}^{c}$ & $\mathrm{NA}^{c}$ & $\mathrm{NA}^{c}$ & 0.114 \\
\hline O4 ‥ H4-C4 (400 ns) & 0.201 & 0.139 & 0.266 & 0.304 & 2.65 & $\mathrm{NA}^{c}$ & 0.306 \\
\hline O4 $\cdots$ H5-C5 (10 ns) & 5.53 & 4.53 & 0.753 & 0.771 & 0.939 & 0.637 & 0.591 \\
\hline 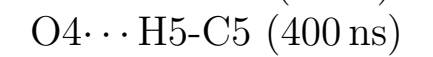 & 8.43 & 932 & 2.58 & 80.8 & 137 & 0.201 & 2.41 \\
\hline O4* H6's-C6 ${ }^{b}(10 \mathrm{~ns})$ & 0.096 & 0.096 & 0.076 & 0.065 & 0.068 & 0.086 & 0.099 \\
\hline 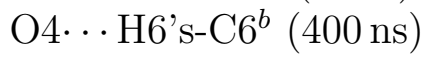 & 0.414 & 0.223 & 0.529 & 0.106 & 0.106 & 0.074 & 0.064 \\
\hline $\mathrm{O} 5 \cdots \mathrm{H} 1-\mathrm{C} 1(10 \mathrm{~ns})$ & $\mathrm{NA}^{c}$ & 17.7 & $\mathrm{NA}^{c}$ & 5.19 & $\mathrm{NA}^{c}$ & $\mathrm{NA}^{c}$ & $\mathrm{NA}^{c}$ \\
\hline O5 $\cdots$ H1-C1 (400 ns) & $\mathrm{NA}^{c}$ & $\mathrm{NA}^{c}$ & $\mathrm{NA}^{c}$ & $\mathrm{NA}^{c}$ & $\mathrm{NA}^{c}$ & $\mathrm{NA}^{c}$ & $\mathrm{NA}^{c}$ \\
\hline $\mathrm{O} 5 \cdots \mathrm{H} 2-\mathrm{C} 2(10 \mathrm{~ns})$ & 0.287 & 0.085 & 0.057 & 0.073 & 0.196 & 0.127 & 0.139 \\
\hline O5 $\cdots$ H2-C2 (400 ns) & 0.077 & 0.314 & 0.384 & 0.223 & 2.44 & 0.065 & 0.109 \\
\hline O5 $\cdots$ H3-C3 (10 ns) & 0.308 & 0.500 & 0.438 & 0.456 & 0.312 & 0.442 & 0.512 \\
\hline O5 $\cdots$ H3-C3 (400 ns) & 0.951 & 2.12 & 0.464 & 0.351 & 0.478 & 0.396 & 0.541 \\
\hline O5 $\cdots \mathrm{H} 4-\mathrm{C} 4(10 \mathrm{~ns})$ & 1.78 & 0.501 & $\mathrm{NA}^{c}$ & $\mathrm{NA}^{c}$ & $\mathrm{NA}^{c}$ & $\mathrm{NA}^{c}$ & $\mathrm{NA}^{c}$ \\
\hline O5 $\cdots$ H4-C4 (400 ns) & 1,612 & 9.91 & 8.62 & $\mathrm{NA}^{c}$ & 0.241 & $\mathrm{NA}^{c}$ & $\mathrm{NA}^{c}$ \\
\hline O5 ‥H5-C5 (10 ns) & 0.410 & 0.377 & 0.365 & 0.504 & 0.816 & 0.364 & 0.350 \\
\hline O5 $\cdots$ H5-C5 (400 ns) & 9.15 & 0.894 & 0.287 & 0.325 & 1.51 & 0.316 & 0.354 \\
\hline O5*H6's-C6 (10 ns) & 0.360 & 0.459 & 0.460 & 0.403 & 0.364 & 0.375 & 0.352 \\
\hline O5 $\cdots \mathrm{H} 6{ }^{\prime} \mathrm{s}-\mathrm{C} 6{ }^{b}$ (400 ns) & 0.498 & 0.596 & 0.576 & 0.366 & 0.312 & 0.447 & 0.402 \\
\hline
\end{tabular}

${ }^{a} \mathrm{~A} \cdots \mathrm{H}-\mathrm{D}=$ hydrogen Acceptor atom $\cdots$ Hydrogen atom-hydrogen Donor atom. ${ }^{22}$

Note: (H-D) share a covalent bond. Data was started at 10.2 and $400.2 \mathrm{~ns}$.

${ }^{b}$ Averaged data

${ }^{c} \mathrm{NA}$ means no hydrogen bonds found at the start of these calculations.

${ }^{d}$ The H's are the HP and HC2, HC3, and HC4 atoms in the TBP molecule.

${ }^{f}$ MDAnalysis H-bond lifetimes do not work with $>100,000$ atoms in the analysis.

For lifetimes without auto-correlation values of zero (i.e., values with $>$ symbol), the final auto-correlation values are $<0.05$ unless otherwise noted: ${ }^{g}<0.1{ }^{h}<0.15$. 
Table (7.14) TBPCl hydrogen bonding lifetimes (ps), Part 5 of 5

\begin{tabular}{|c|c|c|c|c|c|c|c|}
\hline \multirow[b]{2}{*}{$\mathrm{A} \cdots \mathrm{H}-\mathrm{D}^{a}$} & \multicolumn{7}{|c|}{ mol \% water } \\
\hline & 63.1 & 79.4 & 86.8 & 91.1 & 93.9 & 95.8 & 100 \\
\hline O6 $\cdots$ H1-C1 (10 ns) & 11.9 & 31.7 & 0.922 & 0.866 & 0.759 & 0.500 & 0.663 \\
\hline O6 $\cdots$ H1-C1 (400 ns) & 5.91 & 74.9 & 4.51 & 0.826 & 4.83 & 1.03 & 3.87 \\
\hline O6 $\cdots \mathrm{H} 2-\mathrm{C} 2(10 \mathrm{~ns})$ & 0.268 & 0.317 & 0.217 & 0.252 & 0.337 & 0.231 & 0.334 \\
\hline O6 $\cdots \mathrm{H} 2-\mathrm{C} 2$ (400 ns) & 0.424 & 0.552 & 0.797 & 0.531 & 0.641 & 0.416 & 0.433 \\
\hline O6 $\cdots$ H3-C3 (10 ns) & 0.758 & 0.351 & 0.695 & 0.722 & 0.717 & 0.258 & 0.557 \\
\hline O6 $\cdots$ H3-C3 (400 ns) & 1.51 & 8.21 & 0.858 & 1.42 & 38.6 & 0.962 & 0.665 \\
\hline $\mathrm{O} 6 \cdots \mathrm{H} 4-$ & 0.801 & 0.828 & 0.269 & 0.348 & 0.420 & 0.238 & 0.359 \\
\hline $\mathrm{O} 6 \cdots \mathrm{H} 4-$ & 4.94 & 0.717 & 1.03 & 0.417 & 0.686 & 0.323 & 1.15 \\
\hline $\mathrm{O} 6 \cdots \mathrm{H} 5$ & 16.2 & 4.00 & 4.41 & 17.2 & 5.35 & 3.97 & 10.8 \\
\hline O6 $\cdots$ H5-C5 (400n & 15.5 & 2.11 & 2.68 & 2.47 & 3.40 & 6.69 & 4.70 \\
\hline $\mathrm{O} 6 \cdots \mathrm{H} 6{ }^{\prime} \mathrm{s}-\mathrm{C}^{b}(10 \mathrm{~ns})$ & 0.249 & 0.201 & 0.202 & 0.211 & 0.204 & 0.122 & 0.172 \\
\hline $\mathrm{O} 6 \cdots \mathrm{H} 6^{\prime} \mathrm{s}-\mathrm{C}^{b}(400 \mathrm{~ns})$ & 0.343 & 0.370 & 0.271 & 0.434 & 0.316 & 0.216 & 0.275 \\
\hline $\mathrm{Ow} \cdot \mathrm{Hw}-\mathrm{Ow}(10 \mathrm{n}$ & 0.227 & 0.429 & 0.548 & 0.555 & 0.526 & 0.484 & $\mathrm{NA}^{c, f}$ \\
\hline $\mathrm{Ow} \cdots \mathrm{Hw}-\mathrm{Ow}(400 \mathrm{~ns})$ & 0.239 & 0.436 & 0.555 & 0.558 & 0.525 & 0.487 & $\mathrm{NA}^{c, f}$ \\
\hline
\end{tabular}

${ }^{a} \mathrm{~A} \cdots \mathrm{H}-\mathrm{D}=$ hydrogen Acceptor atom $\cdots$ Hydrogen atom-hydrogen Donor atom. ${ }^{22}$ Note: (H-D) share a covalent bond. Data was started at 10.2 and $400.2 \mathrm{~ns}$.

\section{${ }^{b}$ Averaged data}

${ }^{c}$ NA means no hydrogen bonds found at the start of these calculations. ${ }^{d}$ The H's are the $\mathrm{HP}$ and $\mathrm{HC} 2, \mathrm{HC} 3$, and $\mathrm{HC} 4$ atoms in the TBP molecule.

${ }^{f}$ MDAnalysis H-bond lifetimes do not work with $>100,000$ atoms in the analysis. For lifetimes without auto-correlation values of zero (i.e., values with $>$ symbol), the final auto-correlation values are $<0.05$ unless otherwise noted: ${ }^{g}<0.1 ;^{h}<0.15$. 
7.9 Pairwise Energies and Hydrogen Bonding of the Peeling Strands in TBPCl-water (cont.) 


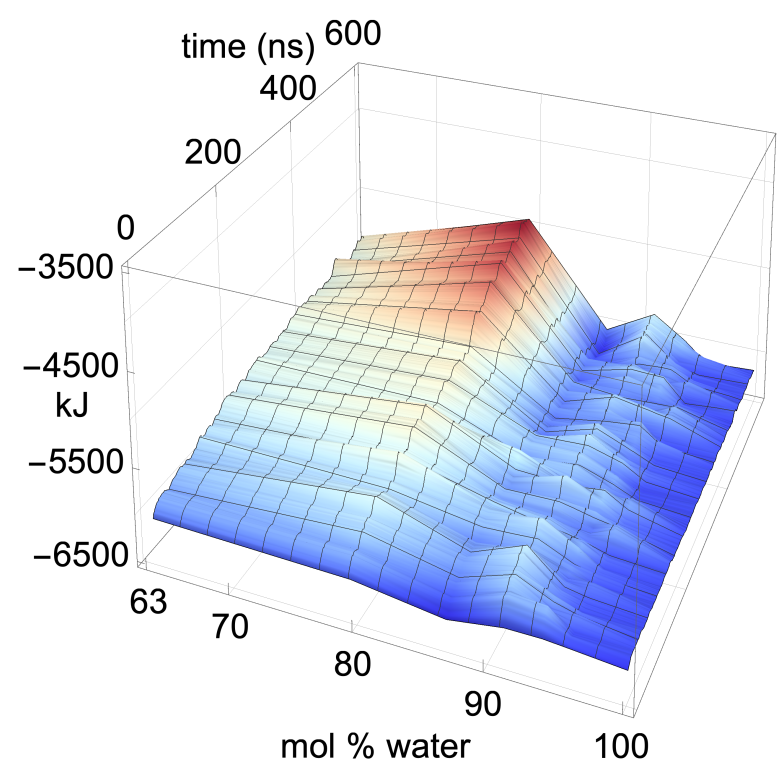

(a) (Bundle and peeling strand)-peeling strand

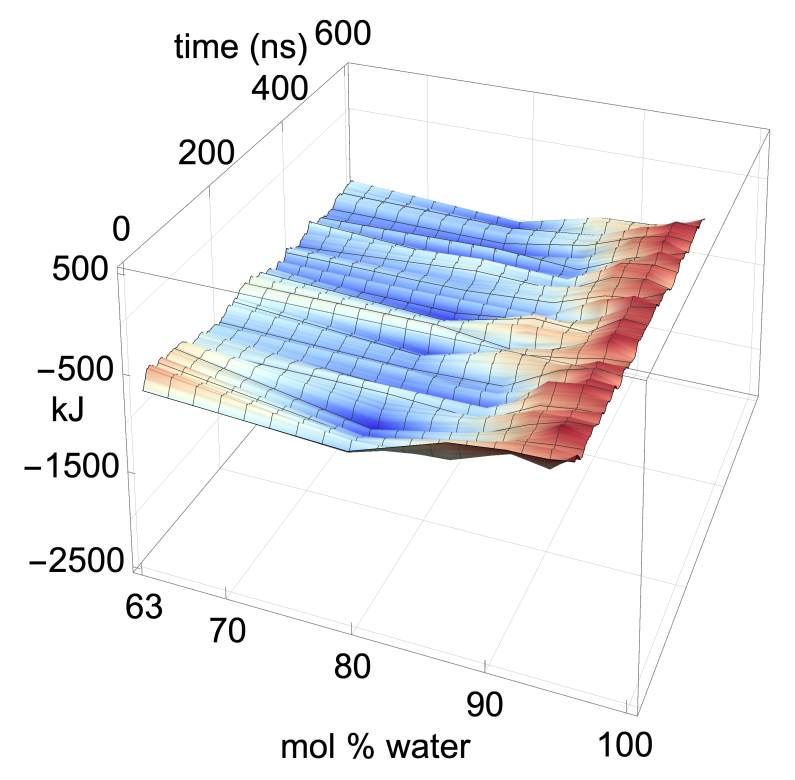

(c) TBP-peeling strand

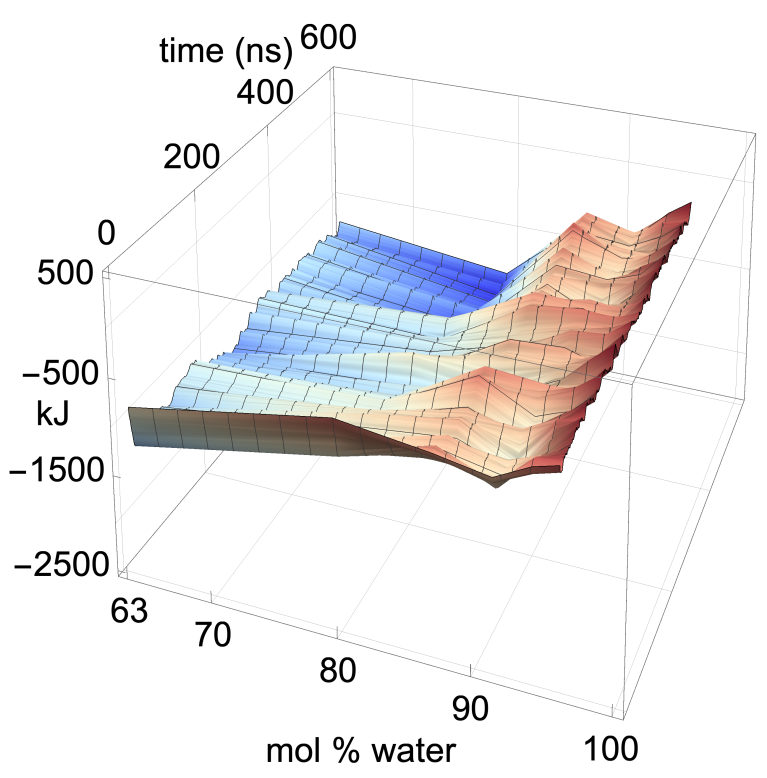

(b) Cl-peeling strand

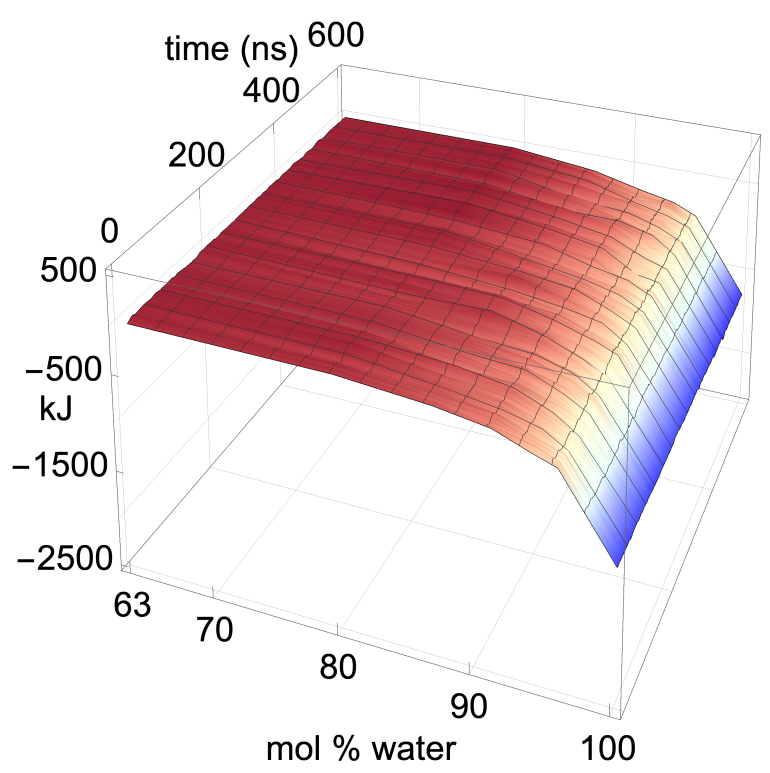

(d) Water-peeling strand

Figure (7.25) The pairwise energies for the second yellow separating strand at $360 \mathrm{~K}$. The pairwise energies are shown between the following: (a) (bundle-peeling strand and peeling strand)-peeling strand (i.e., within the strand and with the rest of the cellulose bundle); (b) Cl-peeling strand; (c) TBP-peeling strand; (d) Water-peeling strand. These data represent the second yellow strand to peel or the non-peeling yellow strand if the second yellow strand does not peel in the simulation. The data were averaged over 1000 data points, using a rolling average. Every $100^{\text {th }}$ point was then plotted to maintain plot clarity. ${ }^{22,23}$ 


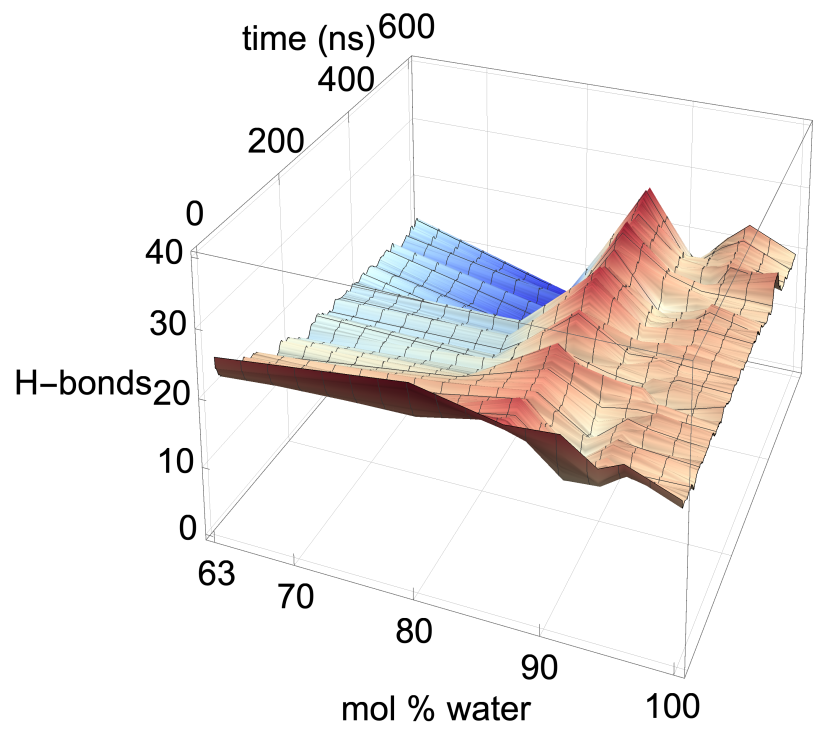

(a) Peeling strand: intra-strand and inter-strand

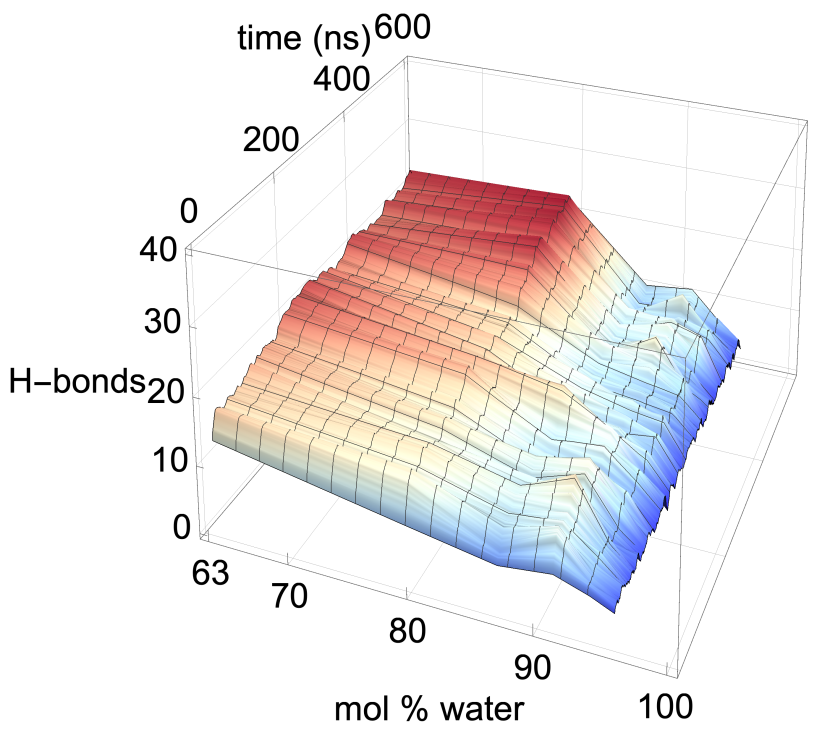

(b) Cl-peeling strand

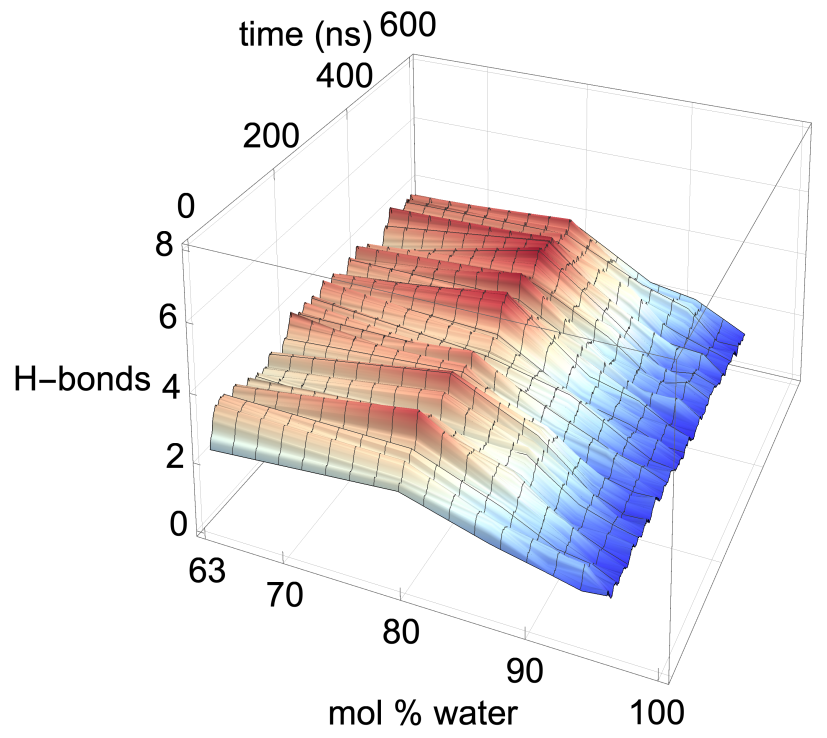

(c) TBP-peeling strand

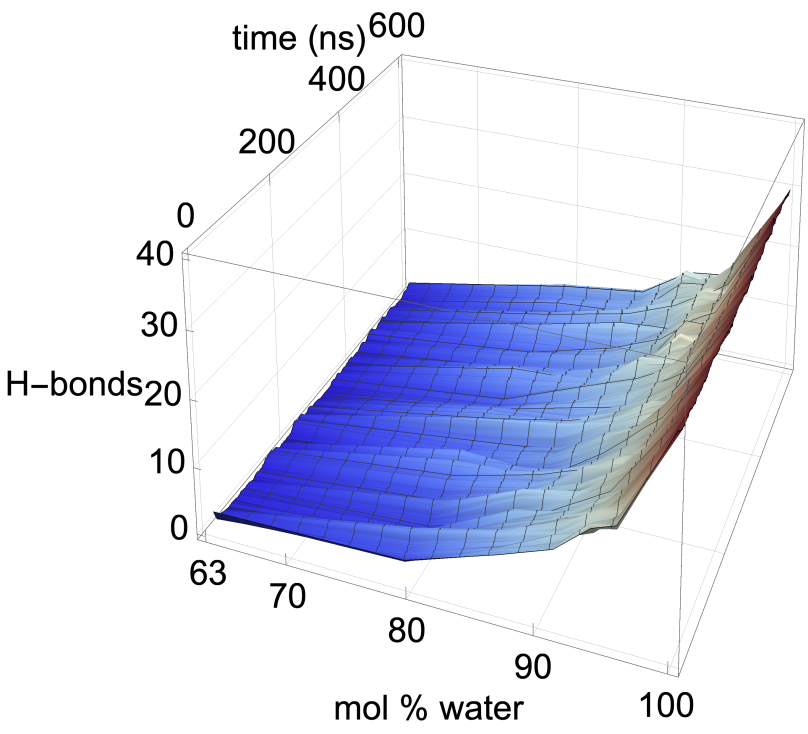

(d) Water-peeling strand

Figure (7.26) The hydrogen bonding for the second yellow separating strand at $360 \mathrm{~K}$ (Part 1 of 2). The hydrogen bonds are shown between the following: (a) (bundle-peeling strand and peeling strand)-peeling strand (i.e., intra-strand and inter-strand hydrogen bonds for the separating strand); (b) Cl-peeling strand; (c) TBP-peeling strand; (d) Water-peeling strand. These data represent the second yellow strand to peel or the non-peeling yellow strand if the second yellow strand does not peel in the simulation. The data were averaged over 1000 data points, using a rolling average. Every $100^{\text {th }}$ point was then plotted to maintain plot clarity. ${ }^{22,23}$ 


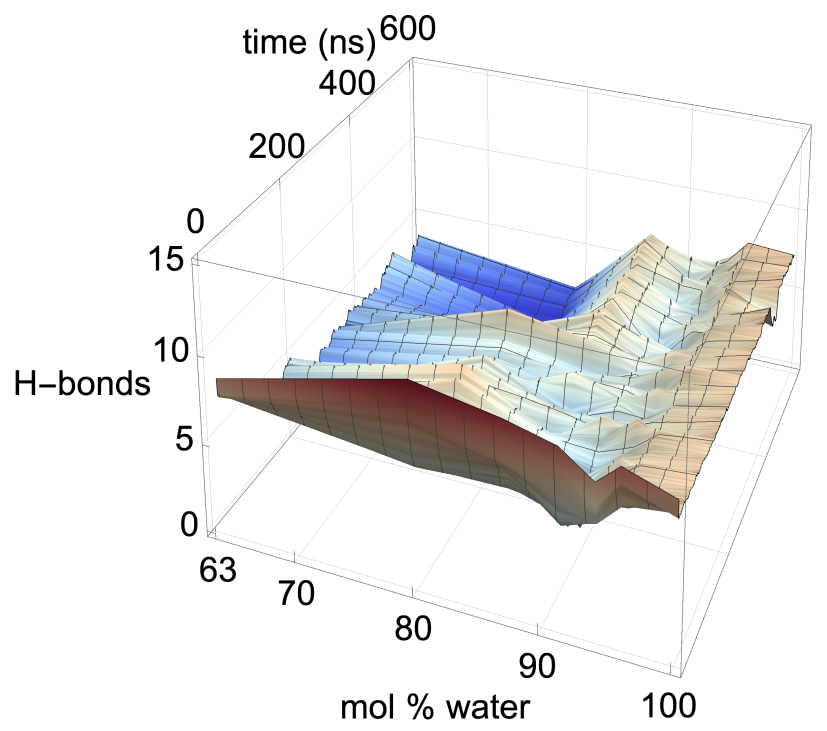

(a) Peeling strand: intra-strand

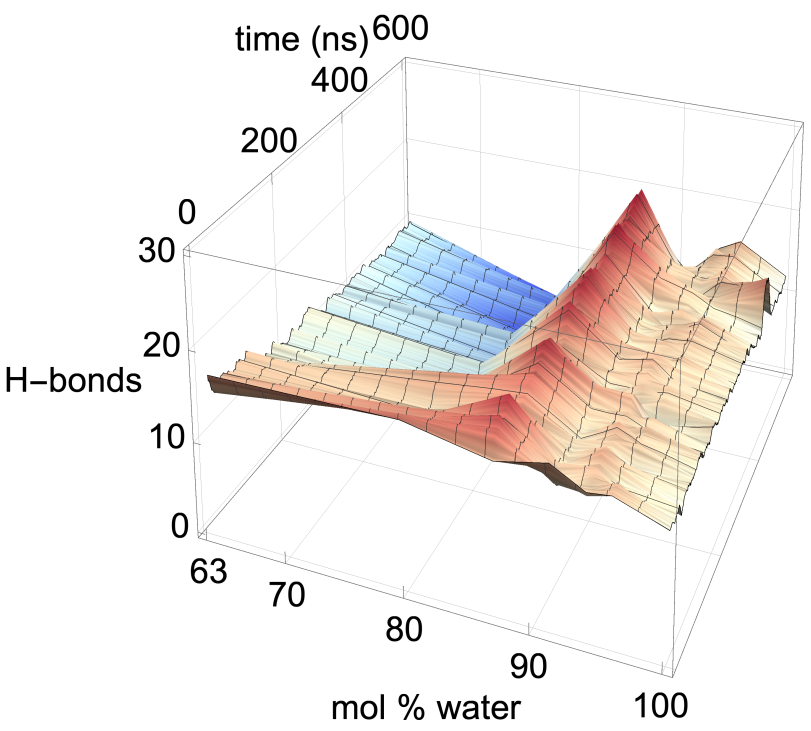

(b) Peeling strand: inter-strand

Figure (7.27) The hydrogen bonding for the second yellow separating strand at $360 \mathrm{~K}$ (Part 2 of 2). The hydrogen bonds are shown between the following: (a) Cellulose: intra-peeling strand (i.e., the intra-strand hydrogen bonds for the separating strand); (b) Cellulose: interpeeling strand (i.e., the inter-strand hydrogen bonds for the separating strand). These data represent the second yellow strand to peel or the non-peeling yellow strand if the second yellow strand does not peel in the simulation. The data were averaged over 1000 data points, using a rolling average. Every $100^{t h}$ point was then plotted to maintain plot clarity. ${ }^{22,23}$ 


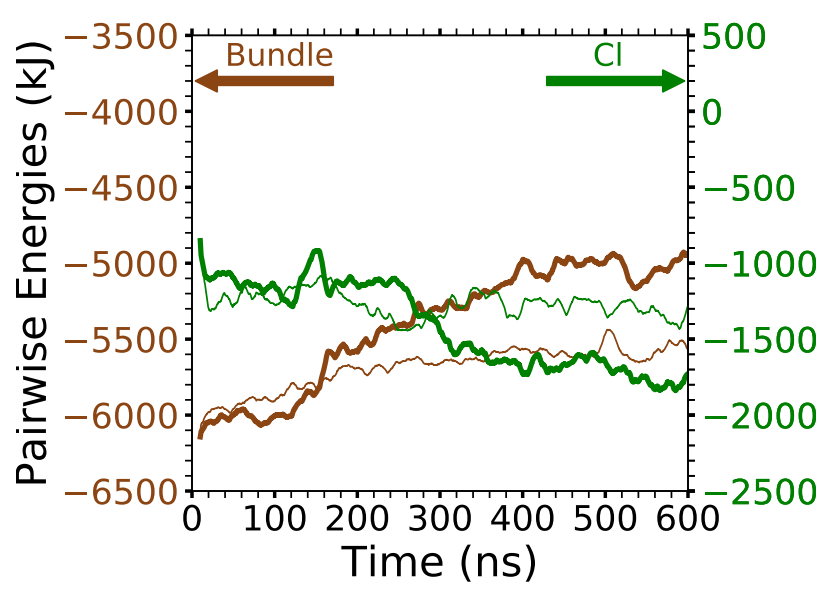

(a) (Cellulose Bundle or $\mathrm{Cl}$ )-Strand

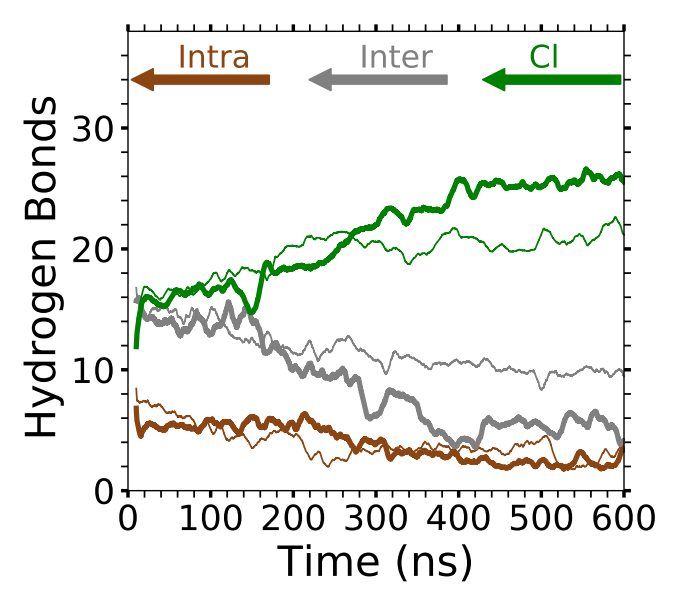

(c) (Intra, Inter, or Cl)-Strand

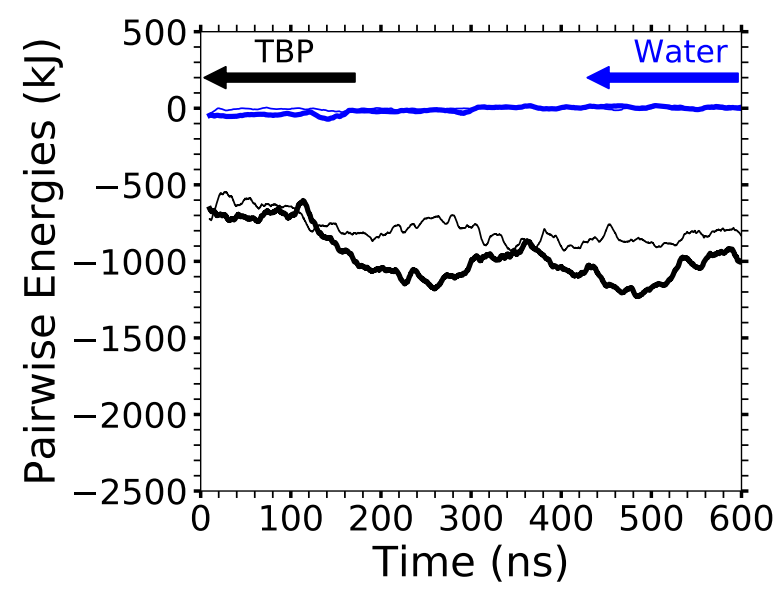

(b) (TBP or Water)-Strand

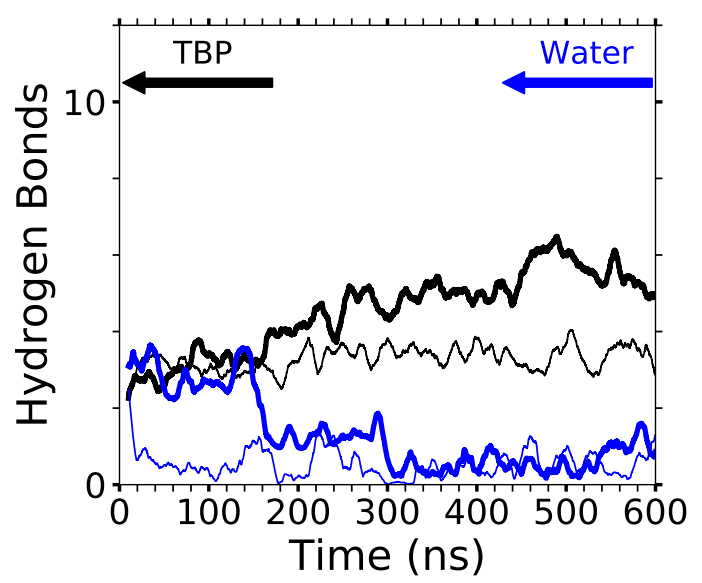

(d) (TBP or Water)-Strand

Figure (7.28) The pairwise energies and hydrogen bonding of the peeling strand(s) at $63.1 \mathrm{~mol} \%$ water and $360 \mathrm{~K}$. The pairwise energies of with the separating strand(s) between the: (a) (Cellulose Bundle or Cl)-Separating Strand; (b) (TBP or Water)-Separating Strand. The number of hydrogen bonds for the separating strand(s) per the given type: (c) (Intra, Inter, or Cl)-Separating Strand; (d) (TBP or Water)-Separating Strand. The thick line is the first yellow strand to peel, and the thin line is the non-peeling yellow strand or second strand to peel. If neither of the stands peel, then both the thick and thin lines are non-peeling yellow strands. The data were averaged over 1000 data points, using a rolling average. $^{22}$ 


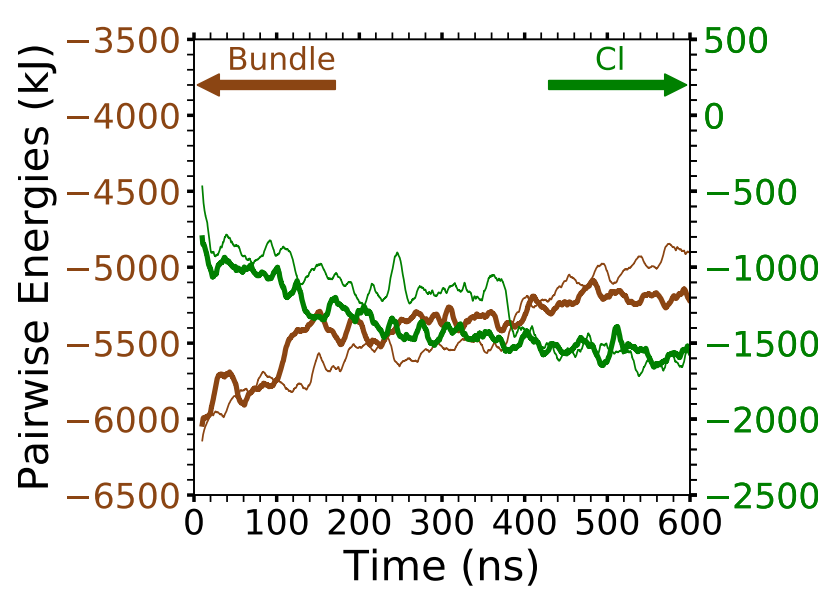

(a) (Cellulose Bundle or $\mathrm{Cl}$ )-Strand

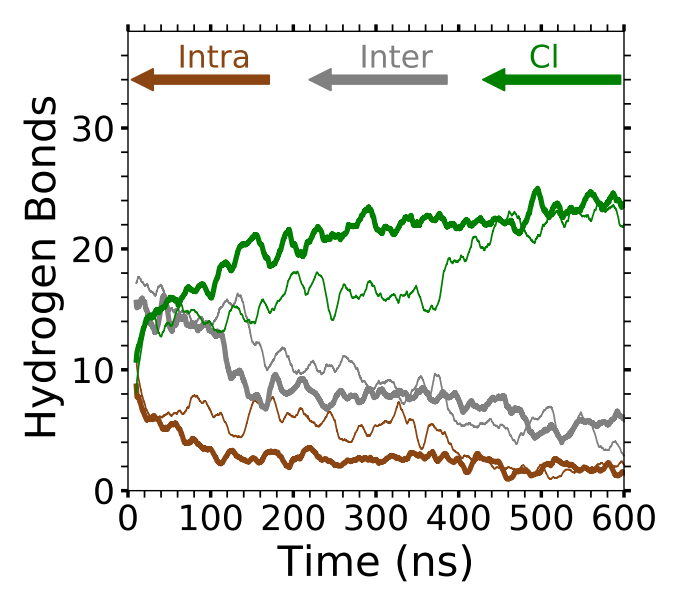

(c) (Intra, Inter, or Cl)-Strand

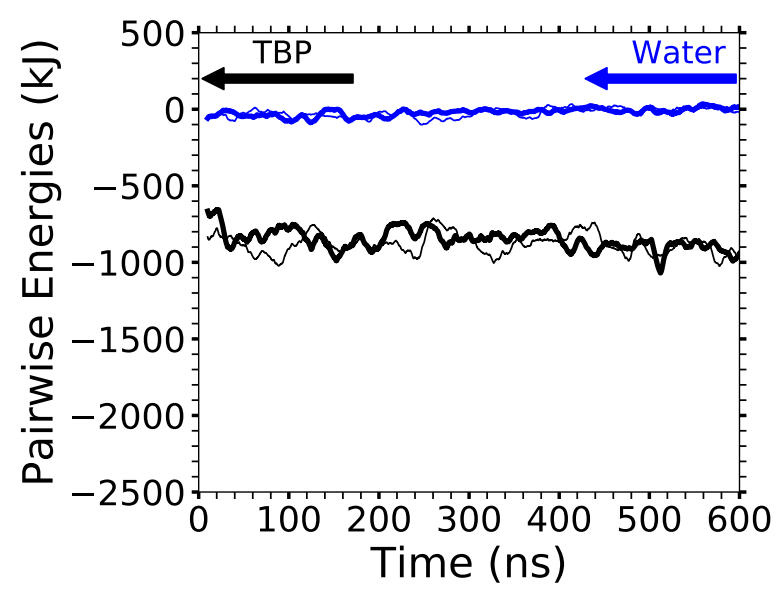

(b) (TBP or Water)-Strand

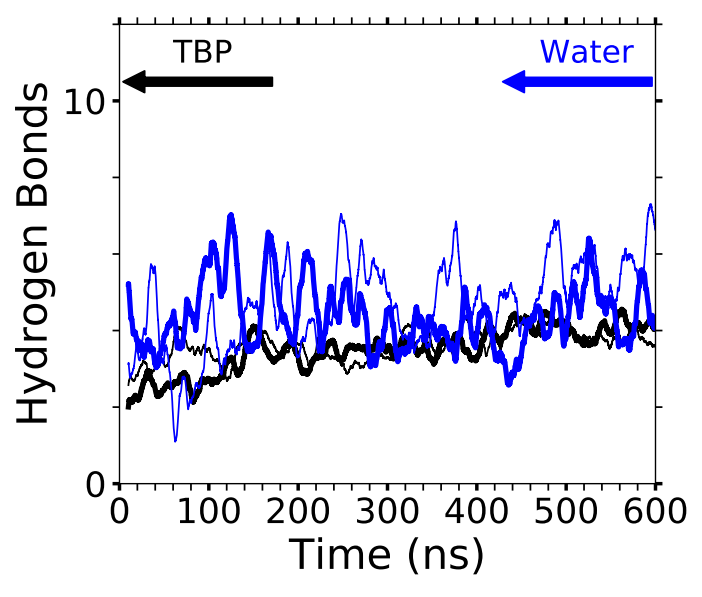

(d) (TBP or Water)-Strand

Figure (7.29) The pairwise energies and hydrogen bonding of the peeling strand(s) at $79.4 \mathrm{~mol} \%$ water and $360 \mathrm{~K}$. The pairwise energies of with the separating strand(s) between the: (a) (Cellulose Bundle or Cl)-Separating Strand; (b) (TBP or Water)-Separating Strand. The number of hydrogen bonds for the separating strand(s) per the given type: (c) (Intra, Inter, or Cl)-Separating Strand; (d) (TBP or Water)-Separating Strand. The thick line is the first yellow strand to peel, and the thin line is the non-peeling yellow strand or second strand to peel. If neither of the stands peel, then both the thick and thin lines are non-peeling yellow strands. The data were averaged over 1000 data points, using a rolling average. ${ }^{22}$ 


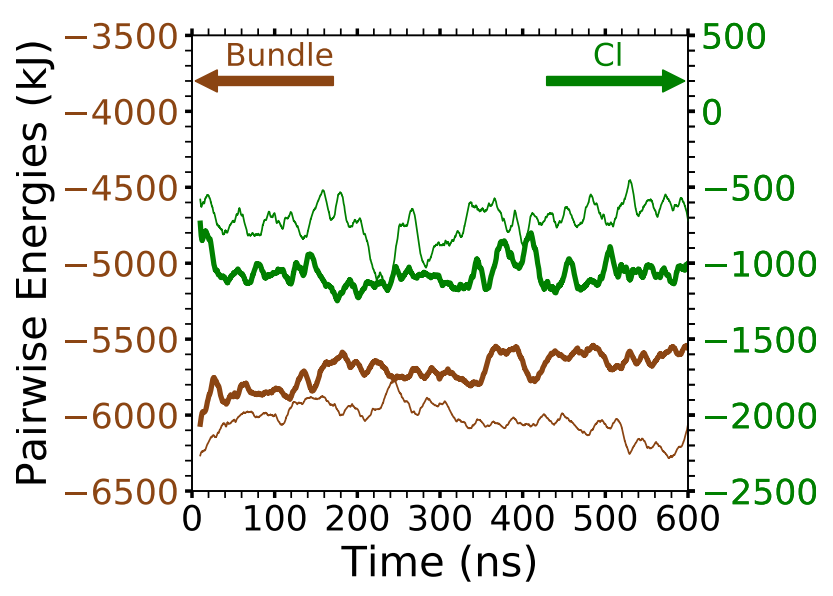

(a) (Cellulose Bundle or $\mathrm{Cl}$ )-Strand

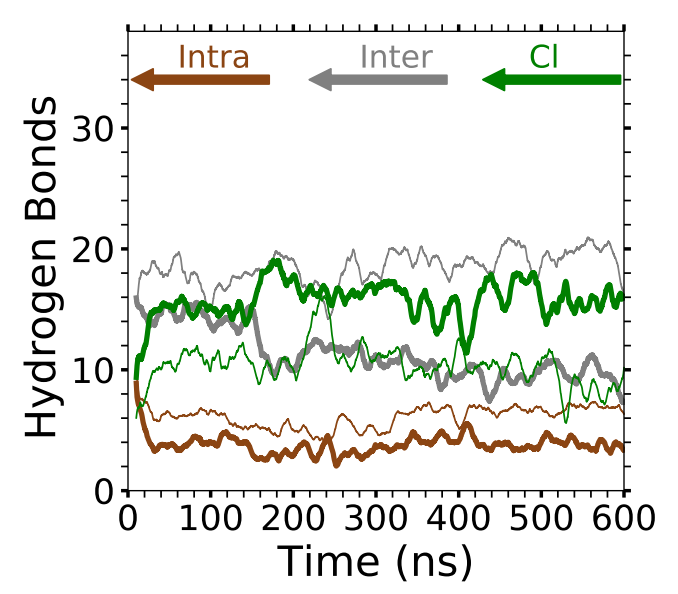

(c) (Intra, Inter, or Cl)-Strand

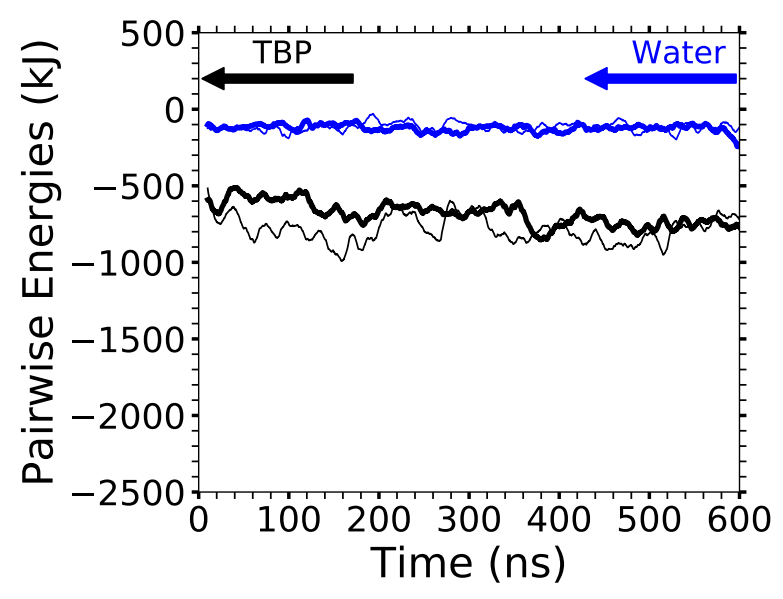

(b) (TBP or Water)-Strand

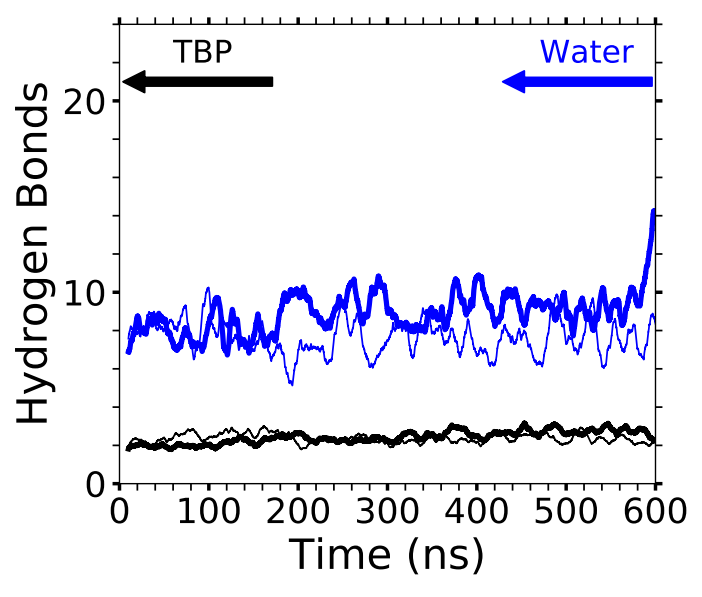

(d) (TBP or Water)-Strand

Figure (7.30) The pairwise energies and hydrogen bonding of the peeling strand(s) at $86.8 \mathrm{~mol} \%$ water and $360 \mathrm{~K}$. The pairwise energies of with the separating strand(s) between the: (a) (Cellulose Bundle or Cl)-Separating Strand; (b) (TBP or Water)-Separating Strand. The number of hydrogen bonds for the separating strand(s) per the given type: (c) (Intra, Inter, or Cl)-Separating Strand; (d) (TBP or Water)-Separating Strand. The thick line is the first yellow strand to peel, and the thin line is the non-peeling yellow strand or second strand to peel. If neither of the stands peel, then both the thick and thin lines are non-peeling yellow strands. The data were averaged over 1000 data points, using a rolling average. $^{22}$ 


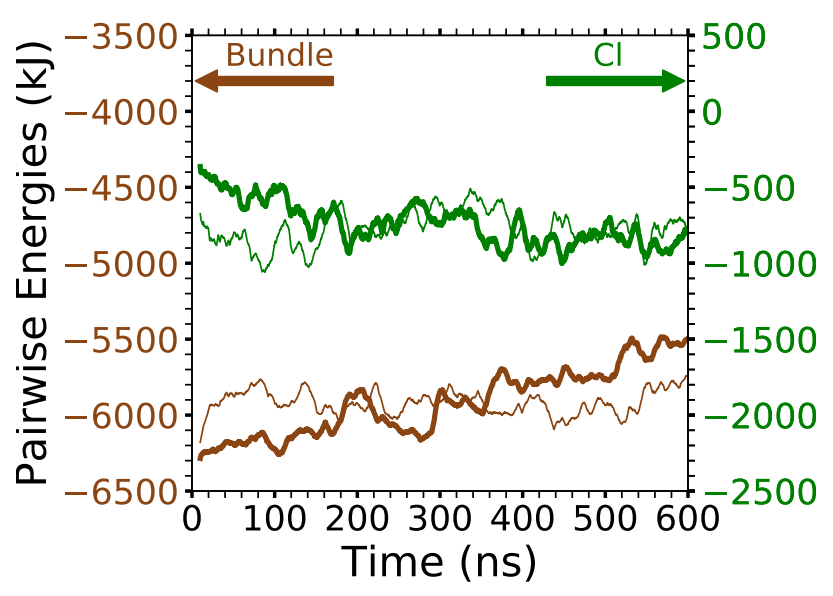

(a) (Cellulose Bundle or $\mathrm{Cl}$ )-Strand

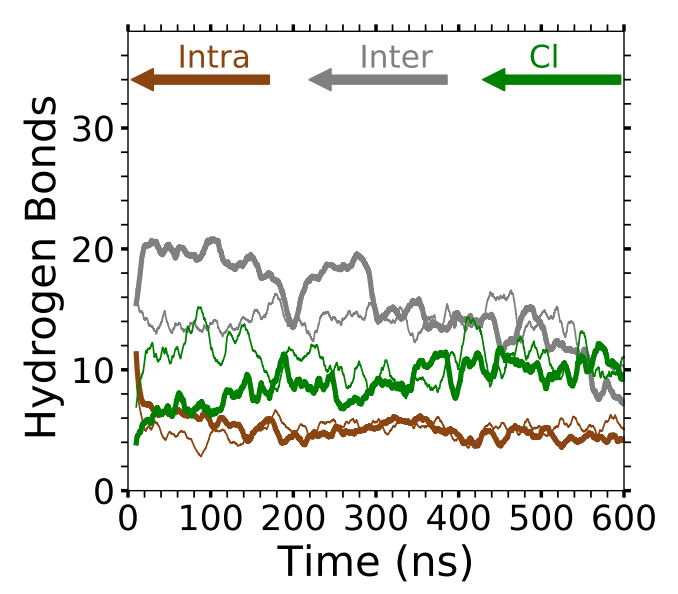

(c) (Intra, Inter, or Cl)-Strand

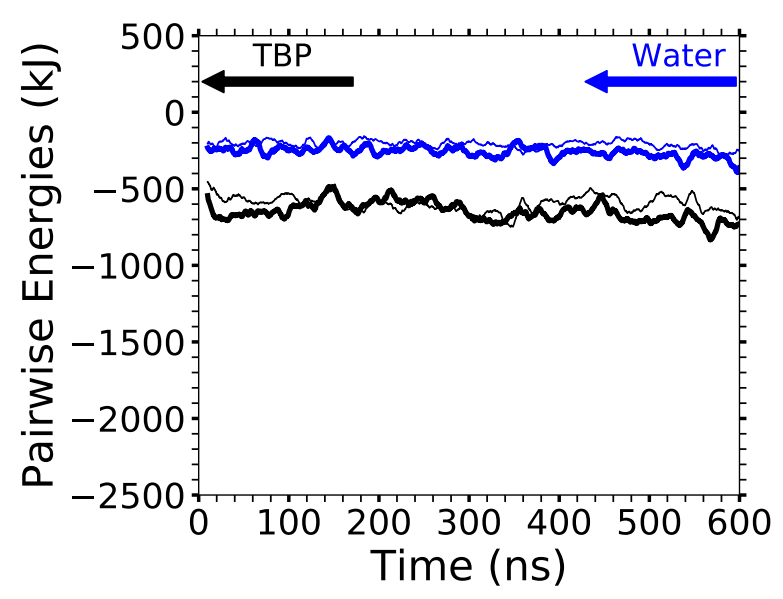

(b) (TBP or Water)-Strand

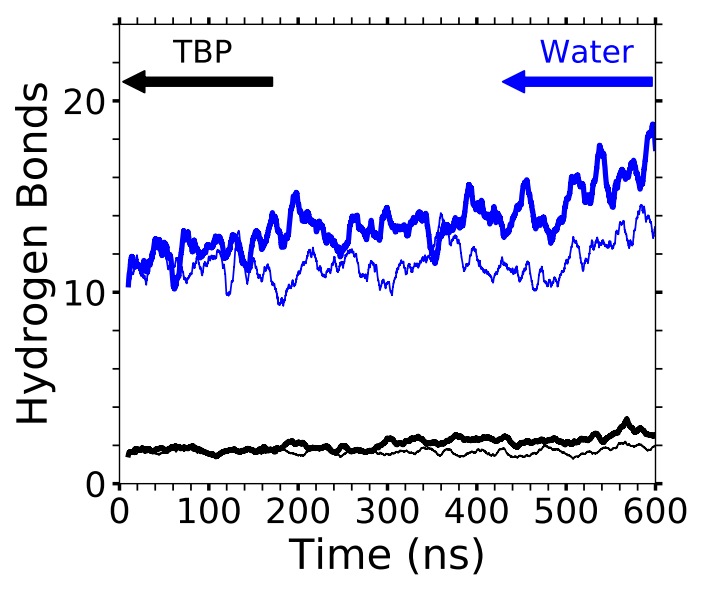

(d) (TBP or Water)-Strand

Figure (7.31) The pairwise energies and hydrogen bonding of the peeling strand(s) at $91.1 \mathrm{~mol} \%$ water and $360 \mathrm{~K}$. The pairwise energies of with the separating strand(s) between the: (a) (Cellulose Bundle or Cl)-Separating Strand; (b) (TBP or Water)-Separating Strand. The number of hydrogen bonds for the separating strand(s) per the given type: (c) (Intra, Inter, or Cl)-Separating Strand; (d) (TBP or Water)-Separating Strand. The thick line is the first yellow strand to peel, and the thin line is the non-peeling yellow strand or second strand to peel. If neither of the stands peel, then both the thick and thin lines are non-peeling yellow strands. The data were averaged over 1000 data points, using a rolling average. ${ }^{22}$ 


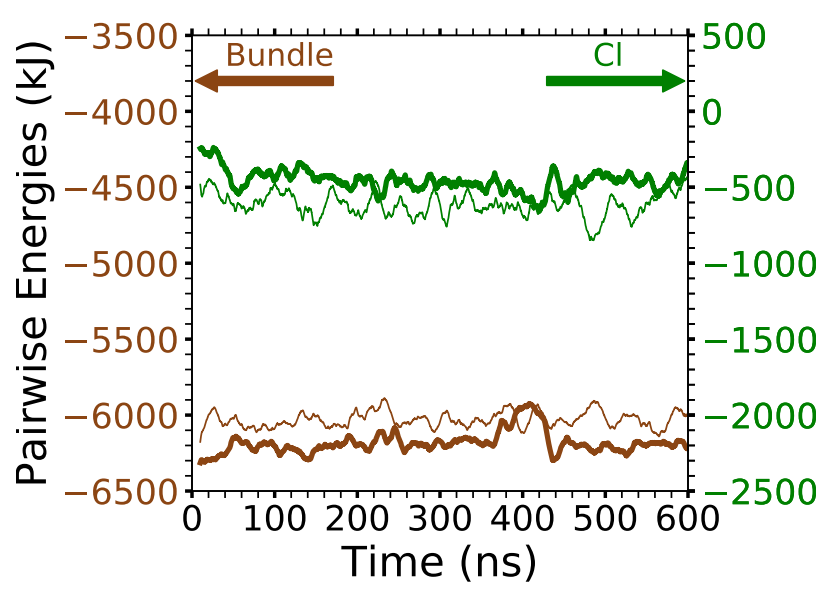

(a) (Cellulose Bundle or $\mathrm{Cl}$ )-Strand

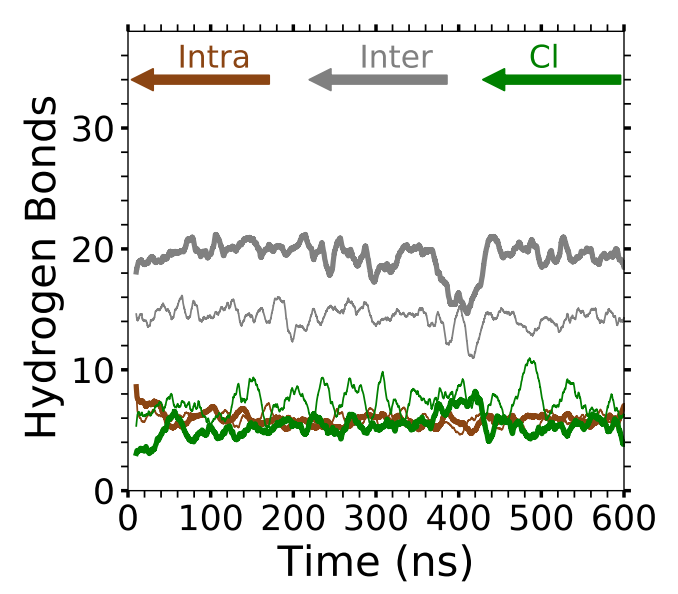

(c) (Intra, Inter, or Cl)-Strand

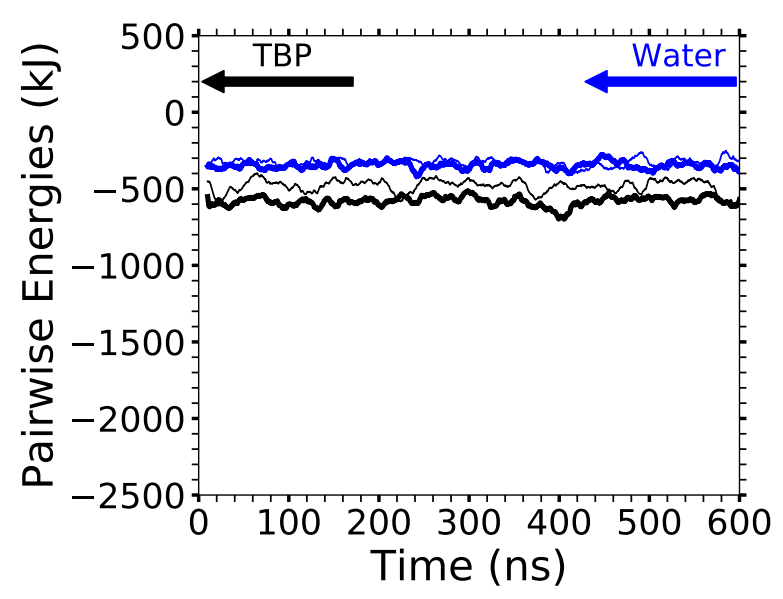

(b) (TBP or Water)-Strand

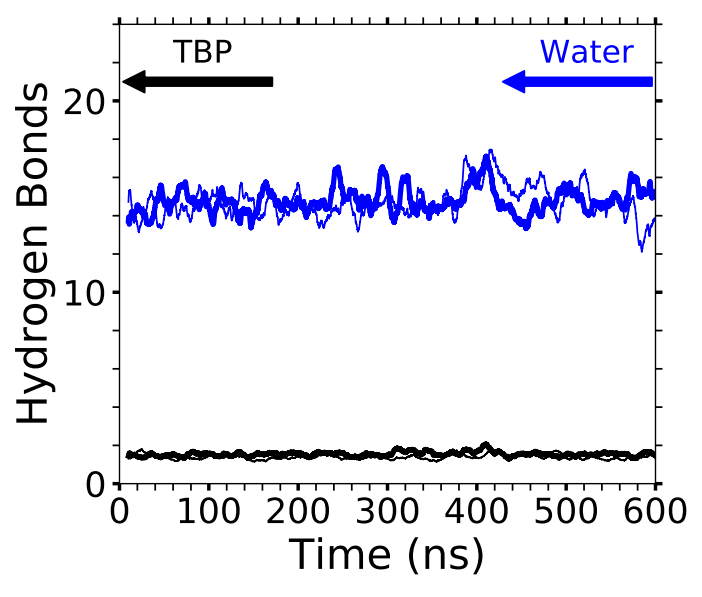

(d) (TBP or Water)-Strand

Figure (7.32) The pairwise energies and hydrogen bonding of the peeling strand(s) at $93.9 \mathrm{~mol} \%$ water and $360 \mathrm{~K}$. The pairwise energies of with the separating strand(s) between the: (a) (Cellulose Bundle or Cl)-Separating Strand; (b) (TBP or Water)-Separating Strand. The number of hydrogen bonds for the separating strand(s) per the given type: (c) (Intra, Inter, or Cl)-Separating Strand; (d) (TBP or Water)-Separating Strand. The thick line is the first yellow strand to peel, and the thin line is the non-peeling yellow strand or second strand to peel. If neither of the stands peel, then both the thick and thin lines are non-peeling yellow strands. The data were averaged over 1000 data points, using a rolling average. ${ }^{22}$ 


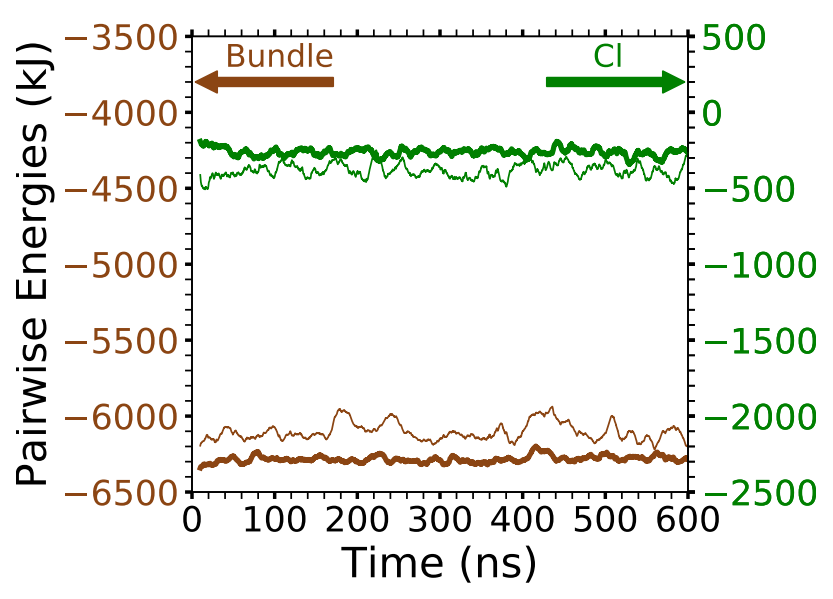

(a) (Cellulose Bundle or $\mathrm{Cl}$ )-Strand

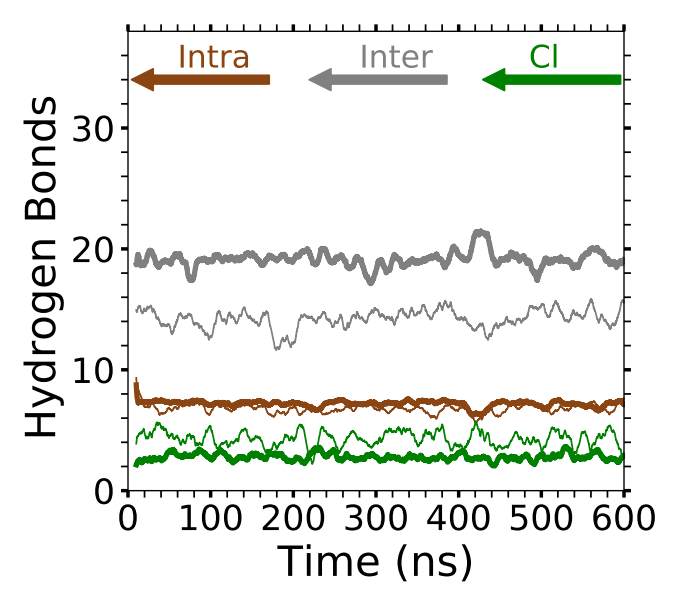

(c) (Intra, Inter, or Cl)-Strand

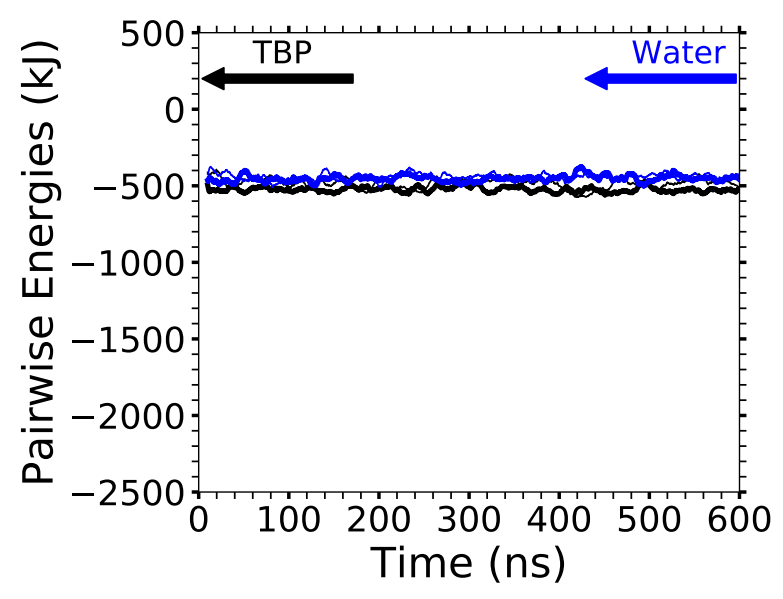

(b) (TBP or Water)-Strand

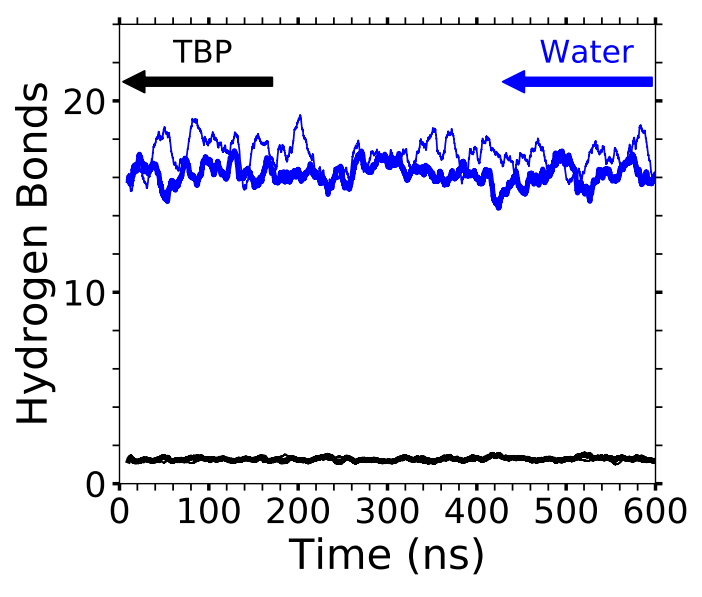

(d) (TBP or Water)-Strand

Figure (7.33) The pairwise energies and hydrogen bonding of the peeling strand(s) at $95.8 \mathrm{~mol} \%$ water and $360 \mathrm{~K}$. The pairwise energies of with the separating strand(s) between the: (a) (Cellulose Bundle or Cl)-Separating Strand; (b) (TBP or Water)-Separating Strand. The number of hydrogen bonds for the separating strand(s) per the given type: (c) (Intra, Inter, or Cl)-Separating Strand; (d) (TBP or Water)-Separating Strand. The thick line is the first yellow strand to peel, and the thin line is the non-peeling yellow strand or second strand to peel. If neither of the stands peel, then both the thick and thin lines are non-peeling yellow strands. The data were averaged over 1000 data points, using a rolling average. ${ }^{22}$ 


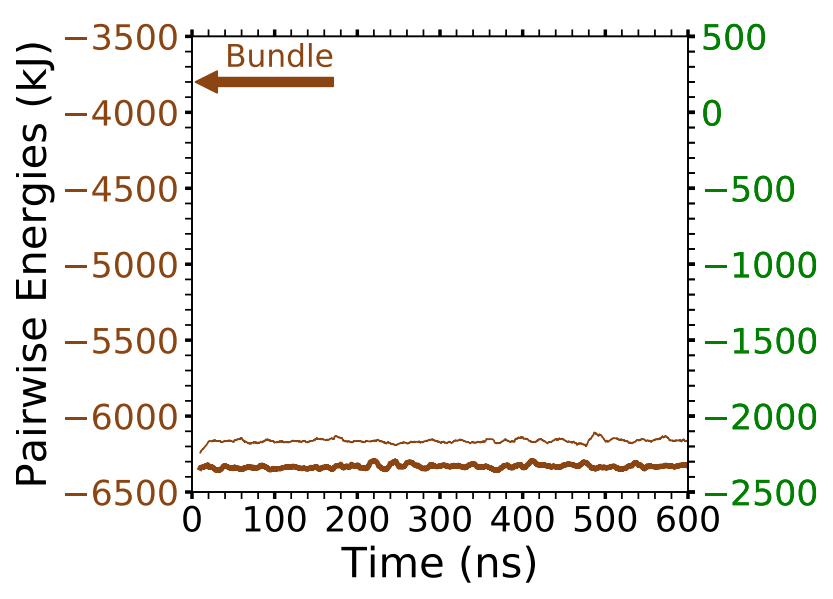

(a) (Cellulose Bundle or $\mathrm{Cl}$ )-Strand

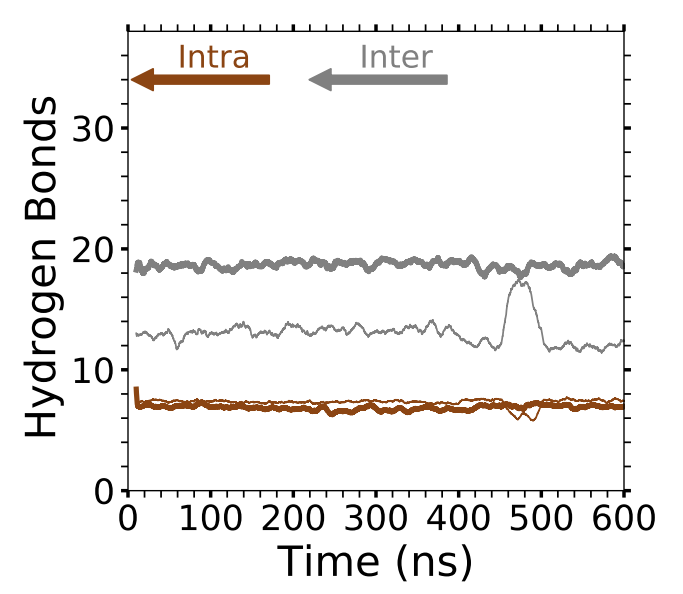

(c) (Intra, Inter, or Cl)-Strand

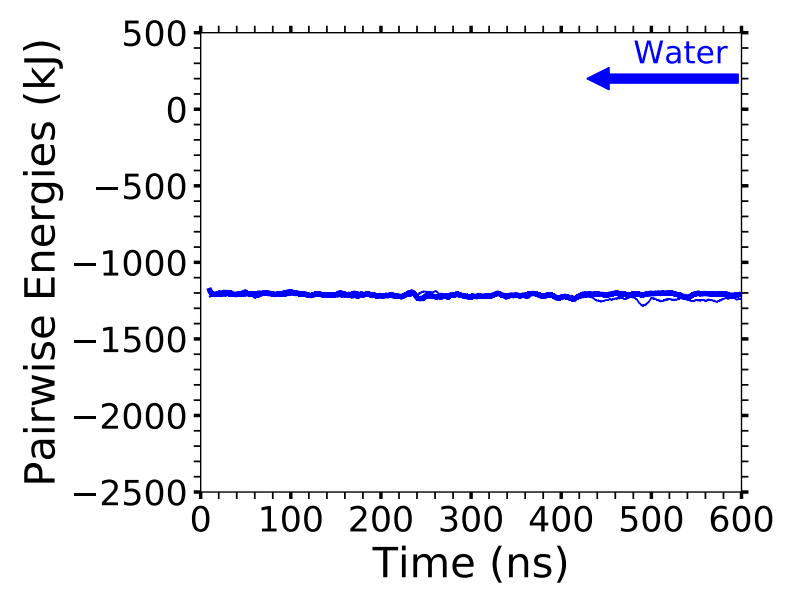

(b) (TBP or Water)-Strand

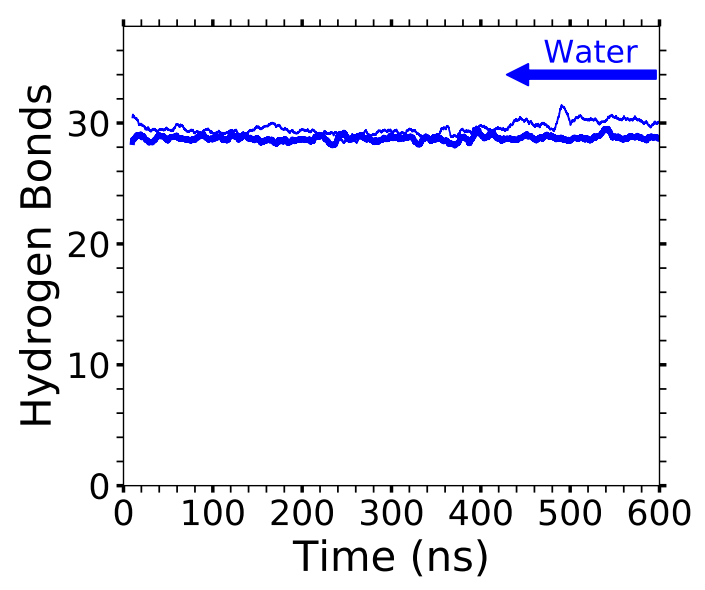

(d) (TBP or Water)-Strand

Figure (7.34) The pairwise energies and hydrogen bonding of the peeling strand(s) at $100 \mathrm{~mol} \%$ water and $360 \mathrm{~K}$. The pairwise energies of with the separating strand(s) between the: (a) (Cellulose Bundle or Cl)-Separating Strand; (b) (TBP or Water)-Separating Strand. The number of hydrogen bonds for the separating strand(s) per the given type: (c) (Intra, Inter, or Cl)-Separating Strand; (d) (TBP or Water)-Separating Strand. The thick line is the first yellow strand to peel, and the thin line is the non-peeling yellow strand or second strand to peel. If neither of the stands peel, then both the thick and thin lines are non-peeling yellow strands. The data were averaged over 1000 data points, using a rolling average. $^{22}$ 


\subsection{Cellulose Dissolution Mechanism in TBPCl-water (cont.)}

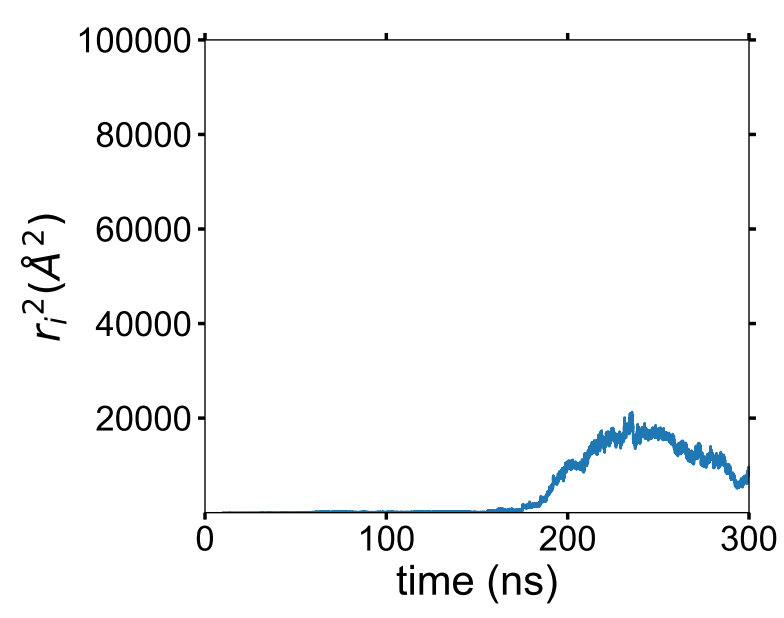

(a) $63.1 \mathrm{~mol} \%$ water: water number 1

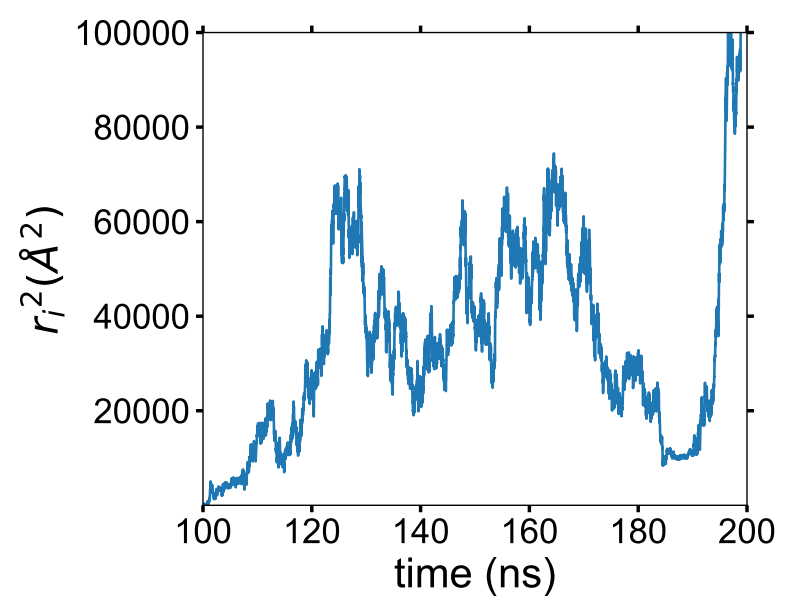

(c) $63.1 \mathrm{~mol} \%$ water: water number 1

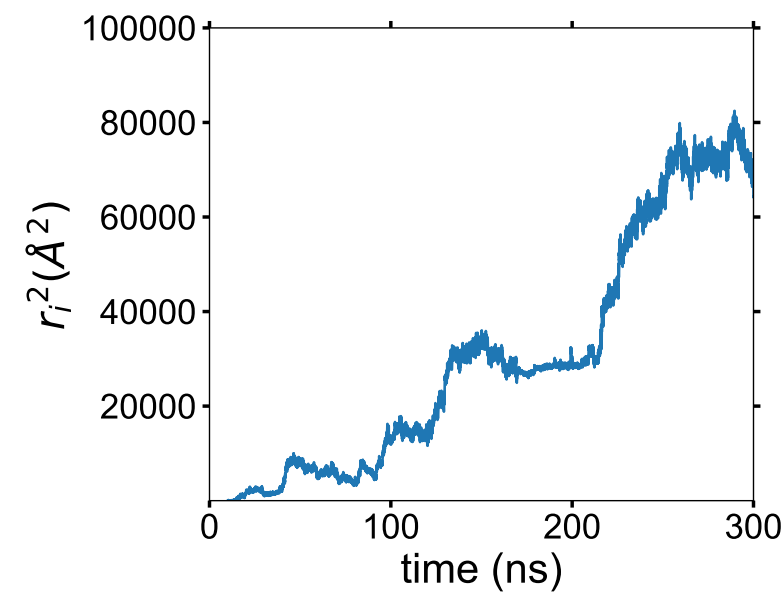

(b) $63.1 \mathrm{~mol} \%$ water: water number 2

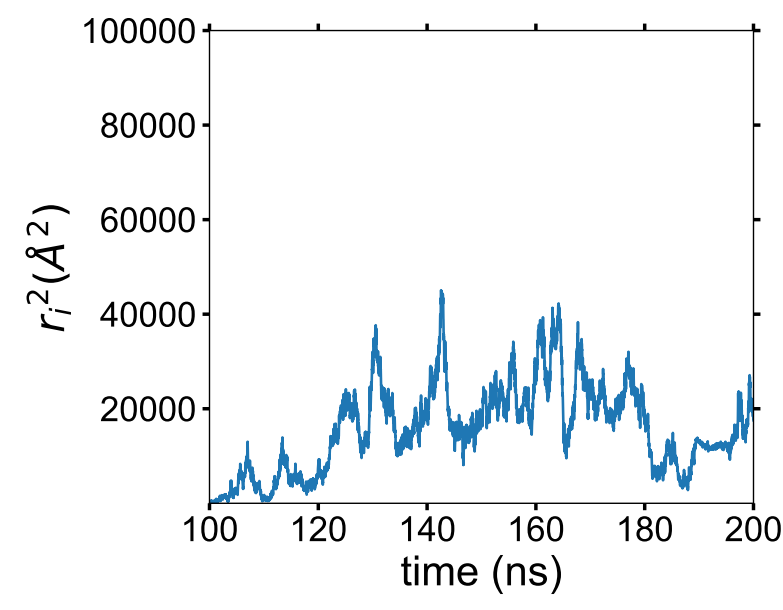

(d) 63.1 mol \% water: water number 2

Figure (7.35) Water diffusion in and out of the pocket between the cellulose strands: (a) water number 1 at the $63.1 \mathrm{~mol} \%$ water concentration; (b) water number 2 at the $63.1 \mathrm{~mol} \%$ water concentration; (c) water number 1 at the $91.1 \mathrm{~mol} \%$ water concentration; (d) water number 2 at the $91.1 \mathrm{~mol} \%$ water concentration. LAMMPS was utilized to calculate the squared displacement $\left(r_{\mathrm{i}}^{2}\right)$ of individual water molecules using the oxygen atom as the center of mass. ${ }^{28}$ The gray shaded area indicates when the water molecule is in the pocket between the cellulose strands. The lack of variation, increase, or decrease in the squared displacement of the water molecule in the gray shaded area shows that the water molecule's mobility can be decreased when it is in the water pocket (i.e., water's diffusion can be decreased when the water molecules are between the cellulose strands). The water molecules show some diffusion in the gray shaded areas, some of which are attributed to the cellulose bundles movement, the water moving with the peeling cellulose strand, or the water moving in the pocket between the cellulose strands. 


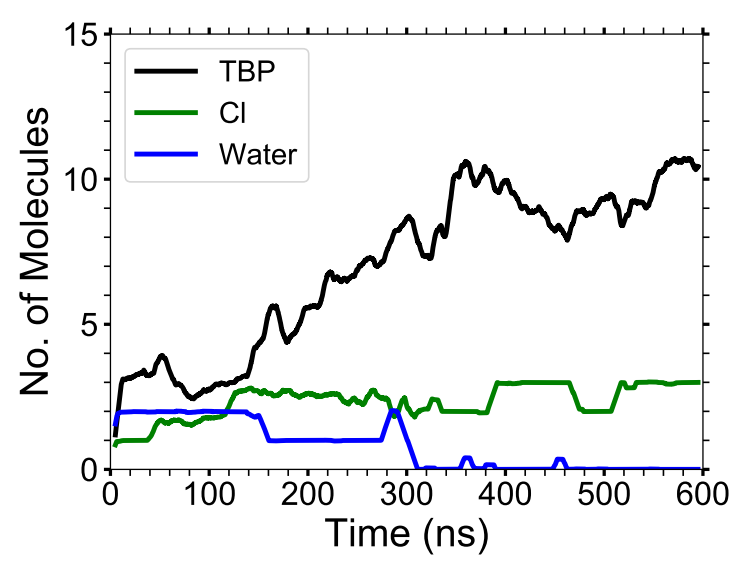

(a) $63.1 \mathrm{~mol} \%$ water

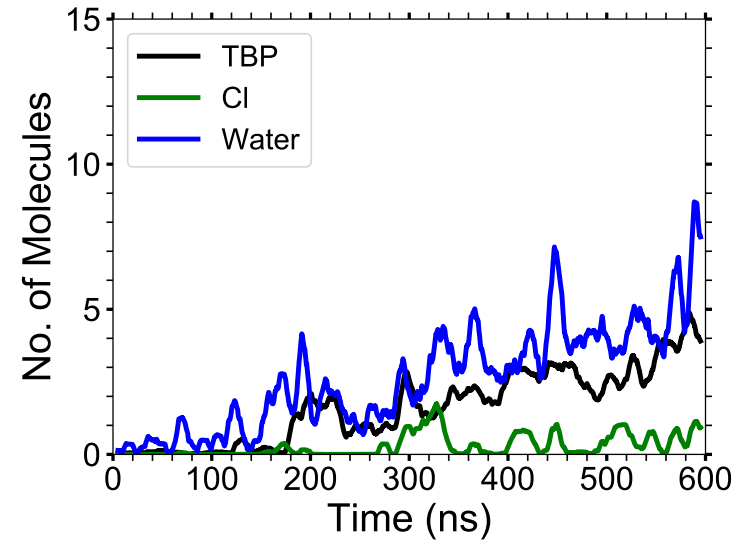

(b) $91.1 \mathrm{~mol} \%$ water

Figure (7.36) The number of molecules between the first yellow peeling strand and the cellulose bundle at various concentrations: (a) $63.1 \mathrm{~mol} \%$ water ; (b) $91.1 \mathrm{~mol} \%$ water. The water, $\mathrm{TBP}$, and $\mathrm{Cl}$ molecules are counted if they are within $3.5 \AA$ of the 10 inner glycans of the light pink interior cellulose strand (see Figure 2.3). The data were averaged over 1000 data points, using a rolling average. 


\section{General Reference to Past Publications Utilized in this Dissertation}

This entire dissertation was written in part or whole by utilizing the following publications, whether it was directly cited in this document or not:

Crawford, B.; Ismail, A. E. Thermodynamic and Transport Properties of Tetrabutylphosphonium Hydroxide and Tetrabutylphosphonium Chloride-Water Mixtures via Molecular Dynamics Simulations. Polymers. 2020, 12(1), 249.

Crawford, B.; Ismail, A. E. Insight into Cellulose Dissolution with the Tetrabutylphospho-

nium Chloride-Water Mixture using Molecular Dynamics Simulation. Polymers. 2020, 12(3), 627. 


\section{References}

(1) Fang, Z., Smith, R. L., Jr., Qi, Z., Eds. Production of Biofuels and Chemicals with Ionic Liquids; Springer: Heidelberg, 2014.

(2) Center for Sustainable Systems, University of Michigan, "Biofuels Factsheet". CSS0809. http://css.umich.edu/factsheets/biofuels-factsheet, 2019.

(3) Energy Information Administration (EIA), Monthly Energy Review, April 2019. 2018.

(4) U.S. DOE, EERE Alternative Fuels Data Center, Ethanol Fuel Basics. 2018.

(5) U.S. Department of Energy (DOE), Energy Efficiency and Renewable Energy (EERE), Biomass Energy Data Book: Edition 4. 2011.

(6) Renewable Fuels Association (RFA), 2019 Annual Industry Outlook. 2019.

(7) The National Biodiesel Board, Plants Listings. 2019.

(8) Burns, F. P.; Themens, P.; Ghandi, K. Assessment of phosphonium ionic liquiddimethylformamide mixtures for dissolution of cellulose. Compos. Interfaces 2013, 21(1), 59-73.

(9) Ren, P.; Ponder, J. W. Dissolution of cellulose in ionic liquid: A review. AIP Conference Proceedings 2017, 1809, 020035.

(10) Tetrabutylphoshonium Chloride 96 wt. $\%$ in $\mathrm{H}_{2} \mathrm{O}$, Tetrabutylphoshonium Chloride (TBPCl), CAS \# 2304-30-5. Sigma-Aldrich: St. Louis, MO, 2018.

(11) Tetrabutylphoshonium Chloride 80 wt. $\%$ in $\mathrm{H}_{2} \mathrm{O}$, Tetrabutylphoshonium Chloride (TBPCl), CAS \# 2304-30-5. TCI AMERICA: Portland, OR, 2014.

(12) Mazza, M.; Catana, D.-A.; Vaca-Garcia, C.; Cecutti, C. Influence of water on the dissolution of cellulose in selected ionic liquids. Cellulose 2009, 16(2), 207-215.

(13) Swatloski, R. P.; Spear, S. J.; Holbrey, J. D.; Rogers, R. D. Dissolution of Cellulose with Ionic Liquids. J. Am. Chem. Soc. 2002, 124(18), 4974-4975. 
(14) Viell, J.; Marquardt, W. Disintegration and dissolution kinetics of wood chips in ionic liquids. Holzforschung 2011, 65(4), 519-525.

(15) Rabideau, B. D.; Agarwal, A.; Ismail, A. E. Observed Mechanism for the Breakup of Small Bundles of Cellulose I and I in Ionic Liquids from Molecular Dynamics Simulations. The Journal of Physical Chemistry B 2013, 117(13), 3469-3479.

(16) Abe, H.; Fukaya, Y.; Ohno, H. Fast and facile dissolution of cellulose with tetrabutylphosphonium hydroxide containing 40 wt \% water. Chem. Commun. 2012, 48, $11808-11810$.

(17) Pinkert, A.; Marsh, K. N.; Pang, S.; Staiger, M. P. Ionic Liquids and Their Interaction with Cellulose. Chemical Reviews 2009, 109(12), 6712-6728.

(18) Chen, X.; Chen, J.; You, T.; Wang, K.; Xu, F. Effects of polymorphs on dissolution of cellulose in $\mathrm{NaOH} /$ urea aqueous solution. Carbohydrate Polymers 2015, 125(10), 85-91.

(19) Wang, H.; Gurau, G.; Rogers, R. D. Ionic liquid processing of cellulose. Chem. Soc. Rev. 2012, 41(4), 1519-1537.

(20) Abe, H.; Kuroda, K.; Ohno, H. Maintenance-Free Cellulose Solvents Based on Onium Hydroxides. ACS Sustainable Chemistry and Engineering 2015, 3(8), 1771-1776.

(21) Chempath, S.; Boncella, J. M.; Pratt, L. R.; Henson, N.; Pivovar, B. S. Density Functional Theory Study of Degradation of Tetraalkylammonium Hydroxides. J. Phys. Chem. C. 2010, 114(27), 11977-11983.

(22) Crawford, B.; Ismail, A. E. Insight into Cellulose Dissolution with the Tetrabutylphosphonium Chloride-Water Mixture using Molecular Dynamics Simulation. Polymers 2020, 12(3), 627.

(23) Inc., W. R. Mathematica, Version 12.0. Champaign, IL, 2019. 
(24) Kirschner, K. N.; Yongye, A. B.; Tschampel, S. M.; GonzálezOuteiriño, J.; Daniels, C. R.; Foley, B. L.; Woods, R. J. GLYCAM06: A generalizable biomolecular force field. Carbohydrates. Journal of Computational Chemistry 2008, 29(4), $622-655$.

(25) Hadden, J. A.; French, A. D.; Woods, R. J. Unraveling Cellulose Microfibrils: A Twisted Tale. Biopolymers 2013, 99(10), 746-756.

(26) Matthews, J. F.; Beckham, G. T.; Bergenstrahle, M.; Brady, J. W.; Himmel, M. E.; Crowley, M. F. Comparison of Cellulose I $\beta$ Simulations with Three Carbohydrate Force Fields. Journal of Chemical Theory and Computation 2012, 8(2), 735-748.

(27) Flyvbjerg, H.; Petersen, H. G. Error estimates on averages of correlated data. J. Chem. Phys. 1989, 91(1), 461-466.

(28) Plimpton, S. J. Fast Parallel Algorithms for Short-Range Molecular Dynamics. J. Comput. Phys. 1995, 117(1), 1-19.

(29) Martinez, L.; Andrade, R.; Birgin, E. G.; Martinez, J. M. Packmol: A package for building initial configurations for molecular dynamics simulations. Journal of Computational Chemistry 2009, 30(13), 2157-2164.

(30) Humphrey, W.; Dalke, A.; Schulten, K. (VMD) (V)isual (M)olecular (D)ynamics. Journal of Molecular Graphics 1996, 14(1), 33-38.

(31) Cornell, W. D.; Cieplak, P.; Bayly, C. I.; Gould, I. R.; Merz, K. M.; Ferguson, D. M.; Spellmeyer, D. C.; Fox, T.; Caldwell, J. W.; Kollman, P. A. A Second Generation Force Field for the Simulation of Proteins, Nucleic Acids, and Organic Molecules. J. Am. Chem. Soc. 1995, 117, 5179-5197.

(32) Lorentz, H. A. Ueber die Anwendung des Satzes vom Virial in der kinetischen Theorie der Gase. Ann. d. Phys. 1881, 12, 127-136.

(33) Berthelot, D. Sur le mélange des gaz. Comptes Rendus Hebd. Acad. Sci. 1898, 126, 1703-1855. 
(34) Canongia Lopes, J. N.; Deschamps, J.; Pádua, A. A. H. Modeling Ionic Liquids Using a Systematic All-Atom Force Field. J. Phys. Chem. B 2004, 108(30), 2038-2047.

(35) Sambasivarao, S.; Acevedo, O. Development of OPLS-AA Force Field Parameters for 68 Unique Ionic Liquids. Journal of Chemical Theory and Computation 2009, 5(4), $1038-1050$.

(36) Zhou, G.; Liu, X.; Zhang, S.; Yu, G.; He, H. A force field for molecular simulation of tetrabutylphosphonium amino acid ionic liquids. J. Phys. Chem. B 2007, 111(25), $7078-7084$.

(37) Isle-Holder, R. E.; W., M.; Hammond, J. R.; Kohlmeyer, A.; Ismail, A. E. Reconsidering Dispersion Potentials: Reduced Cutoffs in Mesh-Based Ewald Solvers Can be Faster than Truncation. Journal of Computational Chemistry 2013, 9(12), 5412-5420.

(38) Posch, H. A.; Hoover, W. G.; Vesely, F. J. Canonical dynamics of the Nosé oscillator: Stability, order, and chaos. Phys. Rev. A 1986, 33(6), 4253-4265.

(39) Hoover, W. G.; Holian, B. L. Kinetic moments method for the canonical ensemble distribution. Physics Letters A 1996, 211(5), 253-257.

(40) Kusnezovab, D.; Bulgacab, A.; Bauerab, W. Canonical ensembles from chaos. Annals of Physics 1990, 204(1), 155-185.

(41) Braga, C.; Travis, K. P. A configurational temperature Nosé-Hoover thermostat. J. Chem. Phys. 2005, 123(13), 134101.

(42) Patra, P.; Bhattacharya, B. A deterministic thermostat for controlling temperature using all degrees of freedom. J. Chem. Phys. 2014, 140(6), 064106.

(43) Nosé, S. A unified formulation of the constant temperature molecular-dynamics methods. Journal of Chemical Physics 1984, 81(1), 511-519.

(44) Hoover, W. G. Canonical dynamics: Equilibrium phase-space distributions. Physical Review A 1895, 31(3), 1695-1697. 
(45) Thijssen, J. M. Computational Physics, 2nd ed.; Cambridge University Press: New York, NY, 2007; pp 1-638.

(46) Verlet, L. Computer Experiments on Classical Fluids. I. Thermodynamical Properties of Lennard-Jones Molecules. Phys. Rev. 1967, 159(1), 98-103.

(47) Ryckaert, J. P.; Ciccotti, G.; Berendsen, H. J. C. Numerical Integration of the Cartesian Equations of Motion of a System with Constraints: Molecular Dynamics of nAlkanes. J. Comput. Phys. 1977, 23(2), 327-341.

(48) Våacha, R.; Megyes, T.; Bakó, I.; Pusztai, L.; Jungwirth, P. Benchmarking Polarizable MD Simulation of Aqueous $\mathrm{NaOH}$ by Diffraction Measurements. J. Phys. Chem. A 2009, 113(16), 4022-4027.

(49) Abascal, J. L. F.; Vega, C. A general purpose model for the condensed phases of water: TIP4P/2005. J. Chem. Phys. 2005, 123(23), 234505.

(50) Hockney, R. W.; Eastwood, J. W. Computer Simulation Using Particles; Taylor and Francis Group: New York, NY, 1988; pp 1-564.

(51) Tuckerman, M.; Berne, B. J.; Martyna, G. J. Reversible multiple time scale molecular dynamics. American Institute of Physics 1992, 97(3), 1990-2001.

(52) Wang, Q.; Keffer, D. J.; Petrovan, S.; Thomas, J. B. Molecular Dynamics Simulation of Poly(ethylene terephthalate) Oligomers. J. Phys. Chem. B 2009, 114(2), 786-795.

(53) Jorgensen, W. L.; Chandrasekhar, J.; Madura, J. D.; Impey, R. W.; Klein, M. L. Comparison of simple potential functions for simulating liquid water. J. Chem. Phys. 1983, 79, 926-935.

(54) Price, D. J.; Brooks, C. L. I. A modified TIP3P water potential for simulation with Ewald summation. J. Chem. Phys. 2004, 121(20), 10096-10103.

(55) Metzler, R.; Jeon, J.-H.; Cherstvy, A. G.; Barkai, E. Anomalous diffusion models and their properties: non-stationarity, non-ergodicity, and ageing at the centenary of single particle tracking. Phys. Chem. Chem. Phys. 2014, 16(44), 24128-24164. 
(56) Grebenkov, D. S. Time-averaged mean square displacement for switching diffusion. Phys. Rev. E 2019, 99(3), 032133.

(57) Hanwell, M.; Curtis, D. E.; Lonie, D. C.; Vandermeersch, T.; Zurek, E.; Hutchison, G. R. Avogadro: an advanced semantic chemical editor, visualization, and analysis platform. Journal of Cheminformatics 2012, 4(17), 1-17.

(58) Crawford, B.; Ismail, A. E. Thermodynamic and Transport Properties of Tetrabutylphosphonium Hydroxide and Tetrabutylphosphonium Chloride-Water Mixtures via Molecular Dynamics Simulations. Polymers 2020, 12(1), 249.

(59) Gomes, T. C. F.; Munir, S. S. CelluloseBuilder: A toolkit for building crystalline structures of cellulose. Journal of Computational Chemistry 2012, 33(14), 1338-1346.

(60) Haughney, M.; Ferrario, M.; McDonald, I. R. Molecular-Dynamics Simulation of Liquid Methanol. J. Phys. Chem. 1987, 91(19), 4934-4940.

(61) Hess, B.; Kutzner, C.; Spoel, D. V. D.; Lindhal, E. GROMACS 4: Algorithms for Highly Efficient, Load-Balanced, and Scalable Molecular Simulation. J. Chem. Theory Comput. 2008, 4(3), 435-447.

(62) Niazi, A. A.; Rabideau, B. D.; Ismail, A. E. Effects of Water Concentration on the Structural and Diffusion Properties of Imidazolium-Based Ionic Liquid-Water Mixtures. J. Phys. Chem. B 2013, 117(5), 1378-1388.

(63) Chowdhuri, S.; Chandra, A. Hydrogen Bonds in Aqueous Electrolyte Solutions: Statistics and Dynamics Based on Both Geometric and Energetic Criteria. Phys. Rev. E 2002, 66(4), 041203.

(64) Chandra, A. Effects of Ion Atmosphere on Hydrogen-Bond Dynamics in Aqueous Electrolyte Solutions. Phys. Rev. Lett. 2000, 85(4), 768-771.

(65) Luzar, A.; Chandler, D. Effect of Environment on Hydrogen Bond Dynamics in Liquid Water. Phys. Rev. Lett. 1996, 76(6), 928-931. 
(66) Luzar, A. Resolving the Hydrogen Bond Dynamics Conundrum. J. Chem. Phys. 2000, 113(23), 10663-10675.

(67) Luzar, A.; Chandler, D. Structure and Hydrogen-Bond Dynamics of Water-Dimethyl Sulfoxide Mixtures by Computer- Simulations. J. Chem. Phys. 1993, 98(10), 81608173.

(68) Luzar, A.; Chandler, D. Hydrogen-bond kinetics in liquid water. Nature 1996, $379(6560), 55-57$.

(69) Nishiyama, Y.; Langan, P.; Chanzy, H. Crystal Structure and Hydrogen-Bonding System in Cellulose I from Synchrotron X-ray and Neutron Fiber Diffraction. Journal of the American Chemical Society 2002, 124(31), 9074-9082.

(70) Gowers, R. J.; Linke, M.; Barnoud, J.; Reddy, T. J. E.; Melo, M. N.; Seyler, S. L.; Dotson, D. L.; Domanski, J.; Buchoux, S.; Kenney, I. M. et al. MDAnalysis: A Python Package for the Rapid Analysis of Molecular Dynamics Simulations. Proceedings of the 15th Python in Science Conference 2016, 102-109.

(71) Michaud-Agrawal, N.; Denning, E. J.; Woolf, T. B.; Beckstein, O. MDAnalysis: A Toolkit for the Analysis of Molecular Dynamics Simulations. Journal of Computational Chemistry 2011, 32(10), 2319-2327.

(72) Gowers, R. J.; Carbone, P. MDAnalysis: A multiscale approach to model hydrogen bonding: The case of polyamide. The Journal of Chemical Physics 2015, 142(22), 224907.

(73) Tetrabutylphoshonium Hydroxide 40 wt. $\%$ in $\mathrm{H}_{2} \mathrm{O}$, Tetrabutylphoshonium Hydroxide (TBPH), CAS \# 14518-69-5. Sigma-Aldrich: St. Louis, MO, 2017.

(74) Tetrabutylphoshonium Hydroxide 40 wt. $\%$ in $\mathrm{H}_{2} \mathrm{O}$, Tetrabutylphoshonium Hydroxide (TBPH), CAS \# 14518-69-5. Fisher Scientific: Fair Lawn, NJ, 2017.

(75) Tetrabutylammonium Hydroxide (TBAH) 54-55 \% aq. solution extrapure AR, Tetrabutylammonium Hydroxide, CAS \# 2052-49-5. Sisco Research Laboratories Pvt. Ltd. (SRL): Maharashtra, India, 2017. 
(76) Tetrabutylammonium Hydroxide 40 wt. $\%$ in $\mathrm{H}_{2} \mathrm{O}$, Tetrabutylammonium Hydroxide (TBAH), CAS \# 2052-49-5. Sigma-Aldrich: St. Louis, MO, 2017.

(77) Tetrabutylammonium chloride 50 wt. $\%$ in $\mathrm{H}_{2} \mathrm{O}$, Tetrabutylammonium chloride (TBACl), CAS \# 1112-67-0. Fisher Scientific: Fair Lawn, NJ, 2018.

(78) Idström, A.; Gentile, L.; Gubitosi, M.; Olsson, C.; Stenqvist, B.; Lund, M.; Bergquist, K.-E.; Olsson, U.; Köhnke, T.; Bialik, E. On the dissolution of cellulose in tetrabutylammonium acetate/dimethyl sulfoxide: a frustrated solvent. Cellulose 2017, $24(9), 3645-3657$.

(79) Hanley, S. J.; Revol, J. F.; Godbout, L.; Gray, D. G. Atomic force microscopy and transmission electron microscopy of cellulose from Micrasterias denticulata; evidence for a chiral helical microfibril twist. Cellulose 1997, 4(3), 209.

(80) Smith, J. M., Van Ness, H. C., Abbott, M. M., Eds. Introduction to chemical engineering thermodynamics, 7th ed.; McGraw-Hill: Boston, MA, 2005.

(81) R. H., P., Green, D. W., Eds. Perry's Chemical Engineers' Handbook 8th Edition; McGraw-Hill: New York, NY, 2008.

(82) Thompson, M. W.; Matsumoto, R.; Sacci, R. L.; Sanders, N. C.; Cummings, P. T. Scalable Screening of Soft Matter: A Case Study of Mixtures of Ionic Liquids and Organic Solvents. J. Phys. Chem. B 2019, 123(6), 1340-1347.

(83) Del Pópolo, M. G.; Voth, G. A. On the Structure and Dynamics of Ionic Liquids. J. Phys. Chem. B 2004, 108(5), 1744-1752.

(84) Takeuchi, H. A jump motion of small molecules in glassy polymers: A molecular dynamics simulation. J. Chem. Phys. 1990, 93(3), 2062.

(85) Müller-Plathe, F. Diffusion of penetrants in amorphous polymers: A molecular dynamics study. J. Chem. Phys. 1991, 94(4), 3192-3199. 
(86) Zhang, W.; van Duin, A. C. T. Second-Generation ReaxFF Water Force Field: Improvements in the Description of Water Density and OH-Anion Diffusion. J. Phys. Chem. B 2017, 121(24), 6021-6032.

(87) Mardoukhi, Y.; Jeon, J. H.; Metzler, R. Geometry controlled anomalous diffusion in random fractal geometries: looking beyond the infinite cluster. Phys. Chem. Chem. Phys. 2015, 17(44), 30134-30147.

(88) Ertl, P.; Bienfait, B. JSME Molecule Editor. https://www.mn-am.com/online_ demos/corina_demo_interactive, 2017.

(89) Sun, X.; Yoo, S.; Xanthesas, S. S.; Dang, L. X. The reorientation mechanism of hydroxide ions in water: A molecular dynamics study. Chem. Phys. Lett. 2009, 481(13), $9-16$.

(90) Gentile, L.; Olsson, U. Cellulose-solvent interactions from self-diffusion NMR. Cellulose 2016, 23(4), 2753-2758.

(91) Medronho, B.; Lindman, B. Competing forces during cellulose dissolution: From solvents to mechanisms. Current Opinion in Colloid and Interface Science 2014, 19(1), $32-40$.

(92) Bialik, E.; Stenqvist, B.; Fang, Y.; Ostlund, A.; Furo, I.; Lindman, B.; Lund, M.; Bernin, D. Ionization of Cellobiose in Aqueous Alkali and the Mechanism of Cellulose Dissolution. J. Phys. Chem. Lett. 2016, 7, 5044-5048.

(93) Rabideau, B. D.; Ismail, A. E. Mechanisms of hydrogen bond formation between ionic liquids and cellulose and the influence of water content. Phys. Chem. Chem. Phys. 2015, 17(8), 5767-5775.

(94) Menjoge, A.; Dixon, J.; Brennecke, J. F.; Maginn, E. J.; Vasenkov, S. Influence of Water on Diffusion in Imidazolium-Based Ionic Liquids: A Pulsed Field Gradient NMR study. J. Phys. Chem. B 2009, 113(18), 6353-6359. 
(95) Zhao, Y.; Liu, X.; Wang, J.; Zhang, S. Insight into the Cosolvent Effect of Cellulose Dissolution in Imidazolium-Based Ionic Liquid Systems. J. Phys. Chem. B 2013, 117, 9042-9049.

(96) Huo, F.; Lui, Z.; Wang, W. Cosolvent or Antisolvent? A Molecular View of the Interface between Ionic Liquids and Cellulose upon Addition of Another Molecular Solvent. J. Phys. Chem. B 2013, 117, 11780-11792.

(97) Gale, E.; Wirawan, R.; Silveira, R.; Pereira, C. S.; Johns, M. A.; Skaf, M. S.; L., S. J. Directed Discovery of Greener Cosolvents: New Cosolvents for Use in Ionic Liquid Based Organic Electrolyte Solutions for Cellulose Dissolution. ACS Sustain. Chem. Eng. 2016, 4, 6200-6207.

(98) Holding, A. J.; Parviainen, A.; Kilpelainen, I.; Soto, A.; King, A. W. T.; Rodriguez, H. Efficiency of hydrophobic phosphonium ionic liquids and DMSO as recyclable cellulose dissolution and regeneration media. Roy. Soc. Chem. 2017, 7, 17451-17461.

(99) Abe, M.; Kuroda, K.; Sato, D.; Kunimura, H.; Ohno, H. Effects of polarity, hydrophobicity, and density of ionic liquids on cellulose solubility. Phys. Chem. Chem. Phys. 2015, 17, 32276-32282.

(100) Govinda, V.; Vasantha, T.; Khan, I.; Venkatesu, P. Effect of the Alkyl Chain Length of the Cation on the Interactions between Water and Ammonium-Based Ionic Liquids: Experimental and COSMO-RS Studies. Indus. Engr. Chem. Res. 2015, 54, 9013-9026.

(101) Remsing, R. C.; Swatloski, R. P.; Rogers, R. D.; Moyna, G. Mechanism of cellulose dissolution in the ionic liquid 1-n-butyl-3-methylimidazolium chloride: a 13C and 35/37Cl NMR relaxation study on model systems. Chem. Commun. 2006, 12, 12711273.

(102) Zhang, C.; Kang, H.; Li, P.; Liu, Z.; Zhang, Y.; Liu, R.; Xiang, J.-f.; Huang, Y. Dual effects of dimethylsulfoxide on cellulose solvating ability of 1-allyl-3-methylimidazolium chloride. Cellulose 2016, 23(2), 1165-1175. 
(103) Weiner, P. K.; Kollman, P. A. AMBER: Assisted model building with energy refinement. A general program for modeling molecules and their interactions. Journal of Computational Chemistry 1981, 2(3), 287-303.

(104) Chempath, S.; Einsla, B. R.; Pratt, L. R.; Macomber, C. S.; Boncella, J. M.; Rau, J. A.; Pivovar, B. S. Mechanism of Tetraalkylammonium Headgroup Degradation in Alkaline Fuel Cell Membranes. The Journal of Physical Chemistry C 2008, 112(9), 3179-3182.

(105) Chowdhury, S.; Mohan, R. S.; Scott, J. L. Reactivity of ionic liquids. Tetrahedron 2007, 63(11), 2363-2389.

(106) Ye, Y.; Elabd, Y. A. Relative Chemical Stability of Imidazolium-Based Alkaline Anion Exchange Polymerized Ionic Liquids. Macromolecules 2011, 44(21), 8494-8503.

(107) Youngs, T. G. A.; Hardacre, C.; Holbrey, J. D. Glucose Solvation by the Ionic Liquid 1,3-Dimethylimidazolium Chloride: A Simulation Study. J. Phys. Chem. B 2007, 111(49), 13765-13774.

(108) Jorgensen, W. L.; Maxwell, D. S.; Tirado-Rives, J. Development and Testing of the OPLS All-Atom Force Field on Conformational Energetics and Properties of Organic Liquids. J. Am. Chem. Soc. 1996, 118(45), 11225-11236.

(109) Robertson, M. J.; Tirado-Rives, J.; Jorgensen, W. L. Improved Peptide and Protein Torsional Energetics with the OPLS-AA Force Field. Journal of Chemical Theory and Computation 2015, 11(7), 3499-3509.

(110) Wei, W.; Meng, F.; Cui, Y.; Jiang, M.; Zhou, Z. Room temperature dissolution of cellulose in tetra-butylammonium hydroxide aqueous solvent through adjustment of solvent amphiphilicity. Cellulose 2017, 24(1), 49-59.

(111) Klein, C.; Sallai, J.; Jones, T. J.; Iacovella, C. R.; McCabe, C.; Cummings, P. T. In Foundations of Molecular Modeling and Simulation: Select Papers from FOMMS 2015, Molecular Modeling and Simulation; Snurr, R., Adjiman, C., Kofke, D., Eds.; Springer: Singapore, 2016. 
(112) Klein, C.; Summers, A. Z.; Thompson, M. W.; Gilmer, J. B.; McCabe, C.; Cummings, P. T.; Sallai, J.; Iacovella, C. R. Formalizing atom-typing and the dissemination of force fields with foyer. Computational Materials Science 2019, 167, 215-227.

(113) Cummings, P. T.; Gilmer, J. B. Open-Source Molecular Modeling Software in Chemical Engineering. Current Opinion in Chemical Engineering 2019, 23, 99-105.

(114) Jankowski, E.; Ellyson, N.; Fothergill, J. W.; Henry, M. M.; Leibowitz, M. H.; Miller, E. D.; Alberts, M. e. a. Perspective on Coarse-Graining, Cognitive Load, and Materials Simulation. Computational Materials Science 109129, 171, 2020.

(115) Dice, B. D.; Ramasubramani, V.; Harper, E. S.; Spellings, M. P.; Anderson, J. A.; Glotzer, S. C. Analyzing Particle Systems for Machine Learning and Data Visualization with Freud. Proceedings of the 18th Python in Science Conference 2019, 27-33. 